\title{
MiniBacillus - the construction of a minimal organism
}

\author{
Dissertation \\ for the award of the degree \\ "Doctor rerum naturalium"
}

at the Georg-August-Universität Göttingen

within the doctoral program

"Microbiology \& Biochemistry"

at the Georg-August University School of Science (GAUSS)

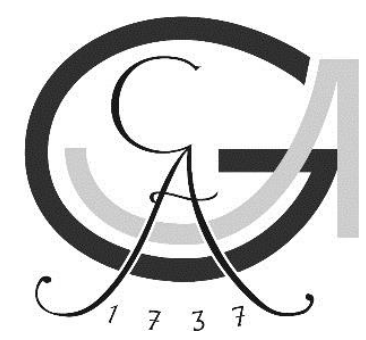

submitted by

Anika Klewing

from Nordhorn 



\section{Thesis Advisory Committee}

Prof. Dr. Jörg Stülke

Institute of Microbiology and Genetics, Department for General Microbiology, University of Göttingen

Prof. Dr. Fabian M. Commichau

Institute of Microbiology and Genetics, Department for General Microbiology, University of Göttingen

Prof. Dr. Kai Heimel

Institute of Microbiology and Genetics, Department of Molecular Microbiology and Genetics, University of Göttingen

\section{Members of the examination board}

Prof. Dr. Gerhard Braus

Institute of Microbiology and Genetics, Department of Molecular Microbiology and Genetics, University of Göttingen

Prof. Dr. Stefanie Pöggeler

Institute of Microbiology and Genetics, Department Genetics of Eukaryotic Microorganisms, University of Göttingen

Prof. Dr. Ivo Feussner

Albrecht von Haller Institute, Department of Plant Biochemistry, University of Göttingen

Date of oral examination: 25 $5^{\text {th }}$ of March, 2019 


\section{Statement of Authorship}

I hereby declare that this doctoral thesis entitled "MiniBacillus- the construction of a minimal organism" has been written independently and with no other sources and aids than quoted.

Anika Klewing 


\section{Danksagung}

Zu allererst möchte ich mich bei Prof. Dr. Jörg Stülke für die Unterstützung und Motivation in den letzten Jahren bedanken. Durch dich konnte ich an einem so spannenden Thema und in einer großartigen Arbeitsgruppe arbeiten.

Prof. Dr. Fabian Commichau danke ich als Teil meines Thesis Committee für die vielen Ideen und Einblicke in den Threonine Metabolismus und das MiniBacillus Projekt. Ein besonderer Dank geht auch an Prof. Dr. Kai Heimel, der immer neue interessante Ideen aus seiner Sichtweise beitragen konnte und darüber hinaus auch immer ein offenes Ohr hatte. Desweiteren danke ich Prof. Dr. Gerhard Braus, Prof. Dr. Stefanie Pöggeler und Prof. Dr. Ivo Feussner für die Teilnahme an der Prüfungskommission.

Ein großes Dankeschön geht an Christina und Larissa. Es hat großen Spaß gemacht, Teil des YbeC Teams zu sein. Christina du motivierst jeden mit deiner positiven Art und deinen guten Ratschlägen. Du bist und bleibst die gute Seele der Abteilung. Larissa, danke für die vielen tollen Mittagessen und den ein oder anderen Sekt.

Danke an Silvia, die mit ihrer Arbeit unseren Alltag um so vieles einfacher macht. Trotzdem bist du immer gut gelaunt und verbreitest deine gute Stimmung bei jedem deiner Rundgänge.

Ich möchte auch meinen Laborkollegen Dominik, Daniel, Jan, Katrin, Martin und Patrick danken. Es hat immer viel Spaß gemacht mit euch im Labor zu stehen und die gegenseitige Unterstützung wird mir fehlen. Auch Bingyao möchte ich für die großartige Hilfe bei jeglicher Datenverarbeitung und sonstigen Problemen danken.

Danke an meine Master- und Bachelorstudenten Dennis Twesmann, Carolin Schunke, Fabian Fiedler und Kolja Otte. Ihr wart eine tolle Unterstützung. Auch allen anderen Kollegen und Studenten der AG Stülke und AG Commichau möchte ich für die tolle Zeit, die Unterstützung und die vielen unvergesslichen Veranstaltungen danken.

Ein großes Dankeschön geht an Miriam. In meiner ersten Labrotation habe ich dich kennengelernt und hätte seitdem bestimmt den einen oder anderen anstrengenden und schwierigen Tag, nicht ohne den Abend in deiner Weinkelterei überstanden.

Cedric, danke für die 9 Jahre Studium die wir zusammen verbracht haben. Auch wenn wir uns manchmal gegenseitig wahnsinnig gemacht haben, haben wir doch immer unseren Spaß gehabt und uns gegenseitig unterstützt.

Danke an meine Mädels für die jahrelange Freundschaft und dafür, dass ihr mich ab und zu wieder auf den Boden der Tatsachen zurückgeholt habt.

Meinen Eltern möchte ich für die jahrelange Unterstützung und den Rückhalt danken. Ihr habt mich immer ermutigt und ohne euch wäre das alles nicht möglich gewesen. Johannes, danke für deine einzigartige Unterstützung und dafür das du immer für mich da bist. 



\section{Table of contents}

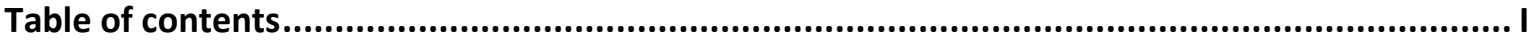

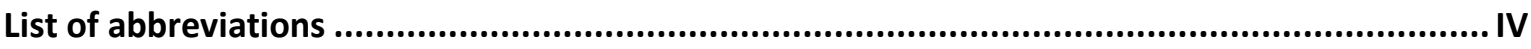

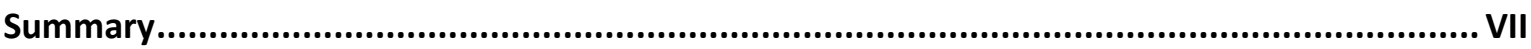

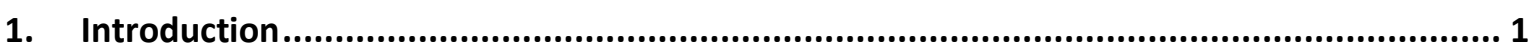

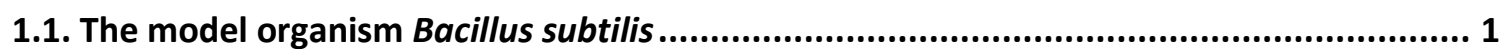

1.2. Minimal genomes and the MiniBacillus project ............................................................ 1

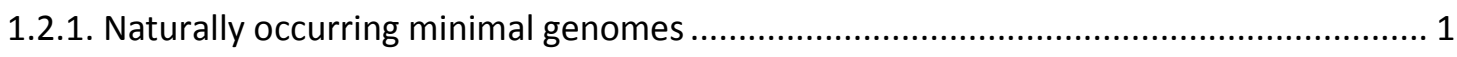

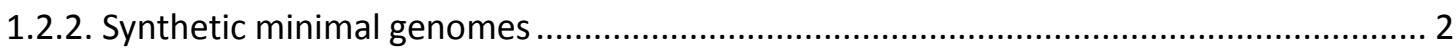

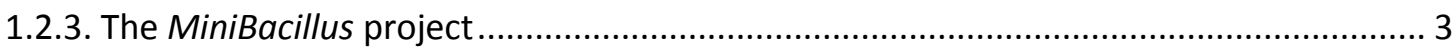

1.3. Central carbon metabolism and the citric acid cycle ................................................ 7

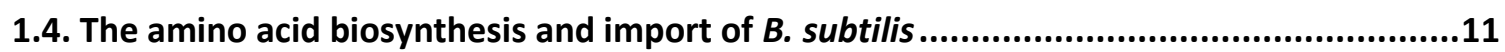

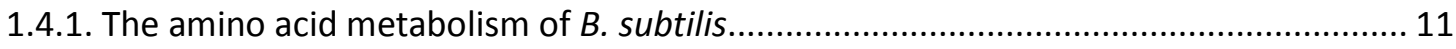

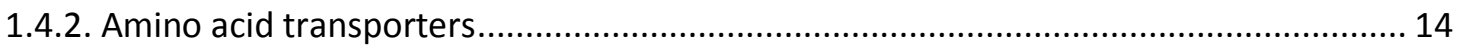

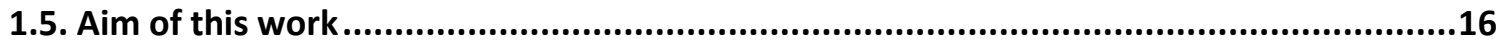

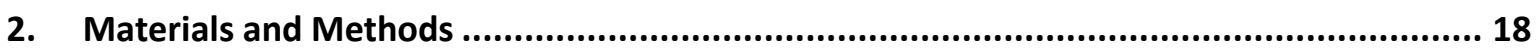

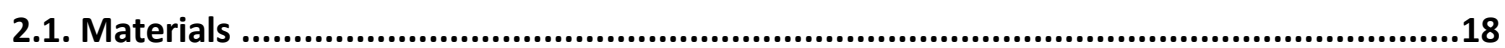

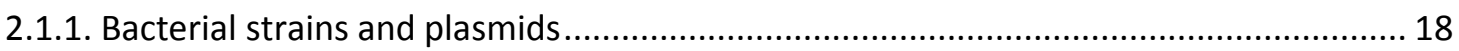

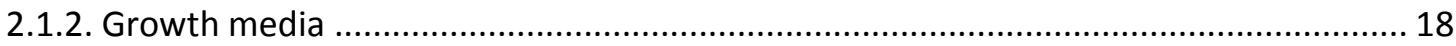

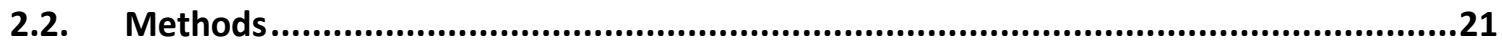

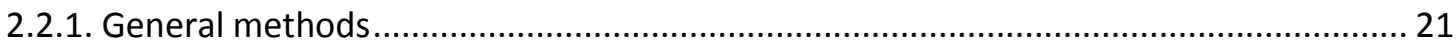

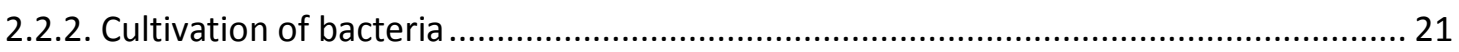

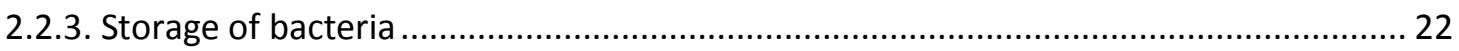

2.2.4. Preparation of competent E. coli and transformation ............................................... 22

2.2.5. Preparation of competent $B$. subtilis cells and transformation ................................... 23

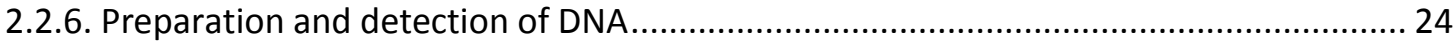

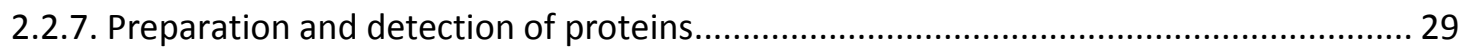




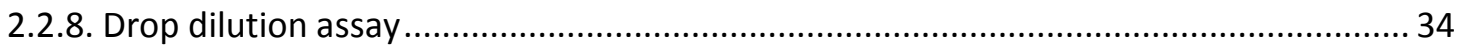

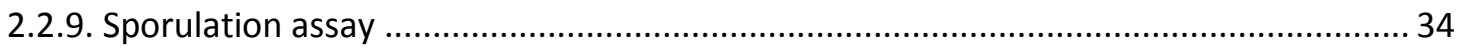

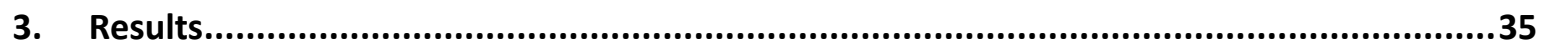

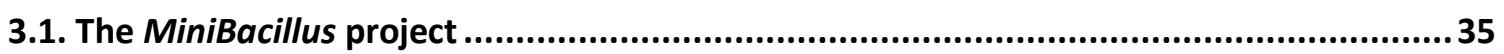

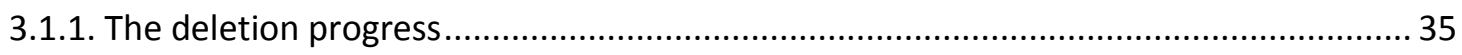

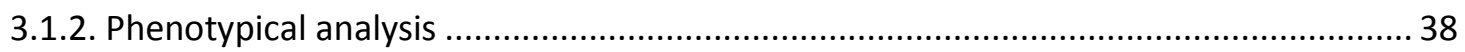

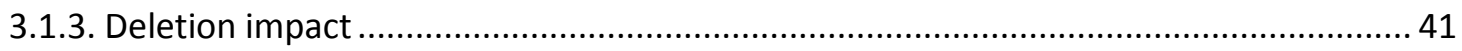

3.2. The role of the TCA cycle in the MiniBacillus project...................................................... 43

3.2.1. The two citrate synthases of Bacillus subtilis ............................................................. 43

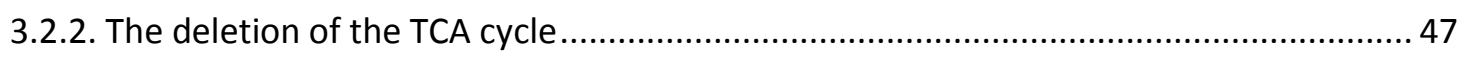

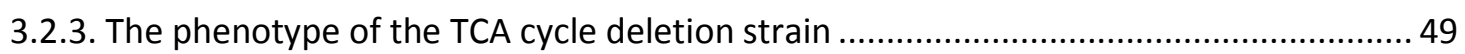

3.3. The identification of serine transporters in Bacillus subtilis .........................................57

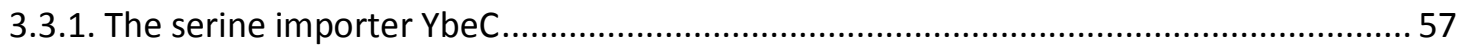

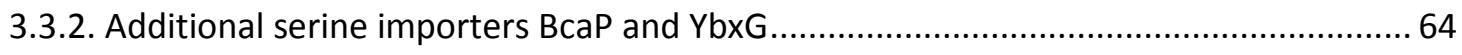

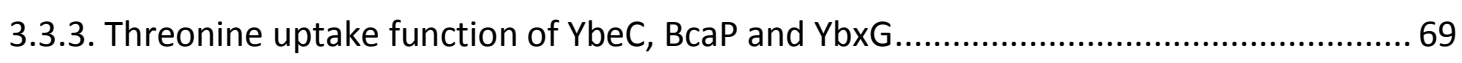

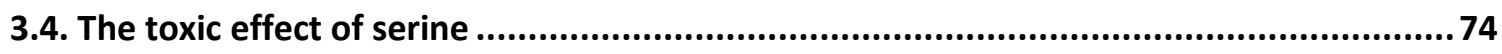

3.5. The consequences for the MiniBacillus project (Blueprint 2.0) ..................................83

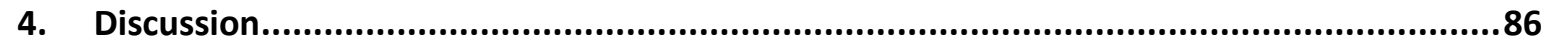

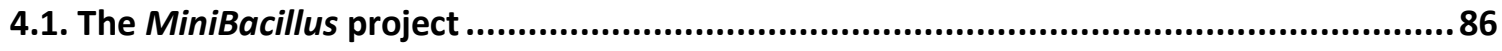

4.2. The functions of the two citrate synthases ....................................................................8

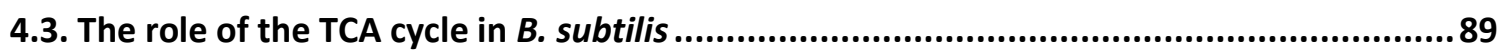

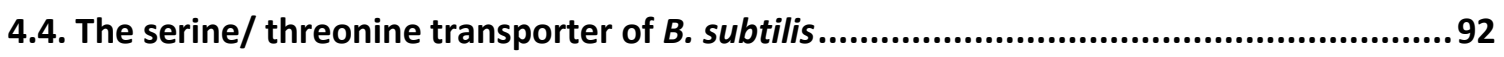

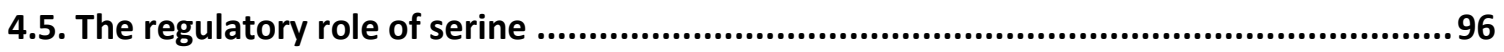

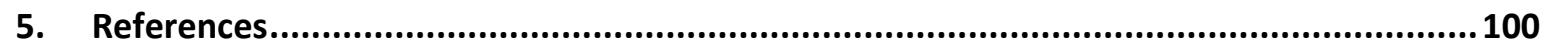

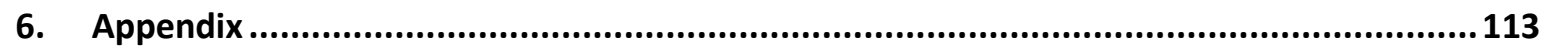

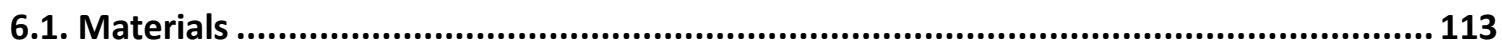

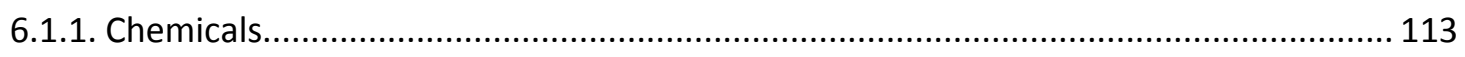

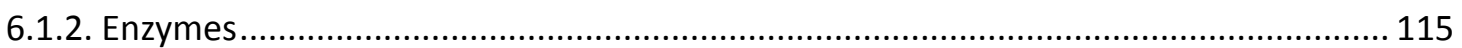


6.1.3. Materials 116

6.1.4. Instruments/ Equipment 116

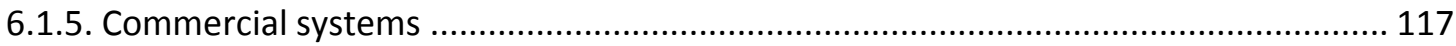

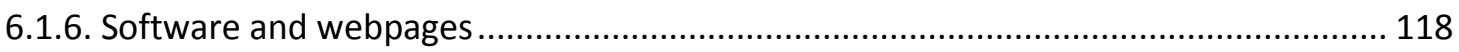

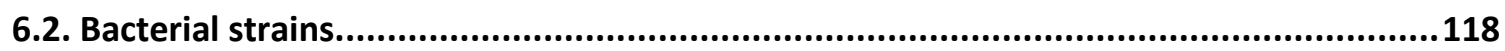

6.2.1. B. subtilis strains constructed in this work.................................................... 118

6.2.2. Isolated suppressor mutants of this work................................................... 128

6.2.3. B. subtilis strains used in this work ........................................................... 128

6.2.4. E. coli strains used in this work ................................................................. 131

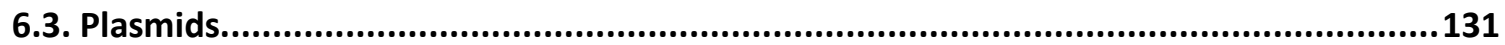

6.3.1. Plasmids constructed in this work.............................................................. 131

6.3.2. Plasmids used in this work ...................................................................... 134

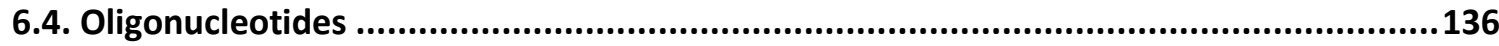

6.4.1. Oligonucleotides constructed in this work................................................. 136

6.4.2. Oligonucleotides used in this work .............................................................. 157

6.5. Supplementary information................................................................... 163

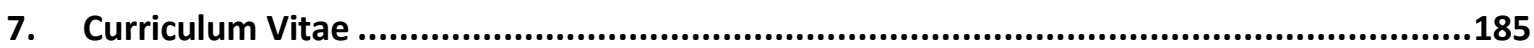




\section{List of abbreviations}

\section{General}

$A B C$

AP

APC

APS

ATP

B.

CAA

cat

CE

CoA

$\mathrm{dH} 2 \mathrm{O}$

DNA

dNTP

E.

e.g.

ECF

erm

et al.

EV

FT

fwd

gDNA

glc

$\mathrm{H}_{2} \mathrm{O}$

IPTG

kan

$\mathrm{kb}$

LB

LFH

M.

$\mathrm{Mb}$

NAD/NADH

NADP/NADPH
ATP-binding cassette

alkaline phosphatase

amino acid-polyamine-organocation

alkaline phosphatase

adenosine triphosphate

Bacillus

casamino acids

chloramphenicol

crude extract

coenzyme A

deionized water

deoxyribonucleic acid

deoxyribonucleosidtriphosphate

Escherichia

It. for example

Energy coupling factor

Erythromycine

et alia

Empty vector

flow through

forward

genomic DNA

glucose

water

isopropyl $\beta$-D-1-thiogalactopyranoside

kanamycin

kilo base pair

lysogeny broth

long flanking homology

Mycoplasma

mega base pairs

nicotinamide adenine dinucleotide

nicotinamide-adeninedinucleotide

phosphate
OD optical density

P promoter

PAGE polyacrylamide gelelectrophoresis

$\mathrm{PCR}$ polymerase chain reaction

$\mathrm{pH} \quad$ power of hydrogen

PTS phosphotransferase system

rev reverse

RNA ribonucleic acid

RNase ribonulease

rpm rounds per minute

RT room temperature

SDS sodium dodecyl sulfate

SP sporulation medium

SSS solute:sodium symporter

TAE tris-acetate-EDTA

TCA tricaboxylic acid

WGS whole genome sequencing

wt wild type 


\section{Amino acids}

Ala

Arg

Asn

Asp

Cys

Gln

Glu

Gly

His

Ile

Leu

Lys

Met

Pro

Phe

Ser

Thr

Trp

Tyr

\section{Prefixes}

$\begin{array}{ll}M & \text { Mega } \\ \mathrm{k} & \text { kilo } \\ \mathrm{m} & \text { milli } \\ \mu & \text { micro } \\ \mathrm{n} & \text { nano } \\ \mathrm{p} & \text { pico }\end{array}$

proline

serine

tyrosine

pico

\section{Units}

${ }^{\circ} \mathrm{C}$ degree Celsius

A ampere

bp base pair

Da Dalton

g gram

h hour

I liter

m meter

$\min$ minute

$\mathrm{mol} \mathrm{mol}$

M molar

s second

v volt

methionine

phenylalanine

threonine

tryptophan 


\section{Summary}

The MiniBacillus project is a minimal genome project. It attempts to reduce the genome of the model organism Bacillus subtilis in a top-down approach by deleting not necessary parts of the genome step by step. In this work, a MiniBacillus strain was created with a genome reduction of $40.51 \%$, which is the most extensive genome reduction, achieved in a top-down approach. The data obtained from previous multi-omics experiments were used to adapt the deletion process and maintain a stable strain. Furthermore, new proteome data were analysed. A glycolytic cassette was inserted as a first attempts of a defragmentation approach to counteract the deceleration of the deletion process. Another important goal of the MiniBacillus project is to gain more knowledge about the mechanisms in the cell. The final minimal cell will be able to utilize glucose as the single carbon source. Therefore, glycolysis and the pentose-phosphate pathway will remain in the cell and the TCA cycle will be deleted. To analyse the effect of a deletion of this central pathway, a TCA cycle mutant in the wild type strain was created. This strain is viable, but shows severe problems in sporulation, competence and cell morphology. Especially the reduced competence is a disadvantage for the MiniBacillus project. The final minimal cell will import all amino acids from the complex medium and all biosynthesis pathways will be deleted. However, not for every amino acid the particular importers are characterized. In this work, the three new serine/ threonine transporters $\mathrm{YbeC}, \mathrm{BcaP}$ and $\mathrm{YbxG}$ could be identified. The $\mathrm{YbeC}$ transporter seems to be the lowaffintiy serine transporter, which transports the major part of serine into the cell at high serine concentrations. BcaP and YbxG have just a minor function. Furthermore, the major import of threonine is mediated by BcaP, which also transports isoleucine and valine into the cell. In contrast, YbeC and YbxG have a minor threonine import function. This information can be used for the MiniBacillus project and the biosynthesis pathways of serine and threonine can be deleted in the final strain. Furthermore, BcaP will remain in the MiniBacillus to ensure the necessary import of serine and threonine.To analyse the serine import, the toxicity of high serine concentrations on wild type cells was utilized. However, the mechanism and physiological role of serine inhibition was unknown. The results of this work indicate a function in the inhibition of the threonine pathway. Serine might bind to the homoserine dehydrogenase protein and inhibit its activity. This causes a reduced level of threonine in the cell. The information of this work was incorporated into the Minibacillus project and a blueprint 2.0 was created. 


\section{Introduction}

\subsection{The model organism Bacillus subtilis}

Bacillus subtilis is a rod-shaped, gram-positive bacterium. It belongs to the phylum Firmicutes, together with, amongst others, Staphylococcus and Clostridium (Wolf et al., 2004). The genome of 4.2 Mbp was completely sequenced in 1997 and harbours around 4100 genes (Kunst et al., 1997). Since the genome of $B$. subtilis is well-studied and many methods are established for the genetic manipulation, it has a high importance as a model organism. Furthermore, B. subtilis is also frequently used for biotechnological applications, e.g. in the production of different products, like riboflavin and vaccines (Hao et al., 2013; Rosales-Mendoza and Angulo, 2015). The wild type strain 168 is a commonly used laboratory strain and harbours a tryptophan auxotrophy (Zeigler et al., 2008). A lot of information gathered about B. subtilis are collected in the SubtiWiki database. It contains e.g. expression and interaction data about all genes and proteins and is therefore an important tool for the work with B. subtilis (Michna et al., 2016). B. subtilis is perfectly adapted to the life in the soil. Its abilities to form spores or biofilms allow the adaption to changing environmental conditions (Piggot and Hilbert, 2004).

\subsection{Minimal genomes and the MiniBacillus project}

\subsubsection{Naturally occurring minimal genomes}

The lifestyle and environmental niches play an important role in the evolution of the genome. Changing environmental conditions or the lack of nutrients lead to a more complex metabolic machinery and therefore to more genes. However, organisms that have adapted to a specific niche with almost constant conditions, show often reduced genomes. These minimal genomes can often be found in pathogenic bacteria. The group of bacteria, called mycoplasma, are organisms with small genomes. Mycoplasma pneumoniae is a human pathogen, causing respiratory infections and it has a genome of around $816 \mathrm{kbp}$ (Himmelreich et al., 1996; Hammerschlag, 2001). Due to the pathogenic lifestyle, many nutrients are provided by the environment and the corresponding biosynthetic pathway are lacking in these bacteria. Furthermore, the bacteria do not form a cell wall (Trachtenberg, 1998). An even smaller genome can be found in the bacterium Mycoplasma genitalium with $580 \mathrm{kbp}$ (Fraser et al., 1995). It was for a long time the smallest known genome, but in 2013 the even smaller genome of Nasuia deltocephalinicola was discovered. This obligate symbiont has a genome of only $112 \mathrm{kbp}$ and is capable to survive without its symbiosis partner the leafhopper (Bennett and Moran, 2013; Ishii et al., 2013). 


\subsubsection{Synthetic minimal genomes}

Minimal genomes are often studied to understand the essence of life. Also synthetic minimal genomes are created and two approaches, top-down and bottom-up, are used for this purpose and the basis is the knowledge about what is essential to sustain life.

In the top-down approach, a naturally occurring genome is reduced stepwise by the deletion of unnecessary regions of the genome. An advantage of this method is that if a problem or an error occurs, it is always possible to get back to the ancestor strain. The error can be easily analysed, since the deletion of one of the genes in this step, must be the reason for this phenotype. An example for a top-down approach is the genome reduced $E$. coli strain created by Hirokawa et al. (2013). This strain showed an improved growth caused by the genome reduction, which could also be beneficial for industrial application.

In a bottom-up approach, in contrast, genomes are synthesised de novo and transplanted into the cell. This approach needs an exact plan of what is needed to sustain life, to assemble the final genome. However, a disadvantage is that errors are not easily identified, since the error occurs only in the final step, the transplantation of the genome. If the cell is not viable, the missing elements need to be identified and the genome needs to be newly synthesized. But if no errors occur, this approach is faster than the top-down approach, since it requires less steps. The creation of a synthetic Mycoplasma cell by the group of Craig Venter is an example for a bottom-up approach. The first milestone in this project was the chemical synthesis of a M. genitalium genome (Gibson et al., 2008). This was followed by the first transplantation of a M. mycoides genome into a M. capricolum cell, which shows that the general principle of genome transplantation is possible (Lartigue et al., 2007). These techniques were then applied to create the strain JCVI-syn3.0, a M. mycoides offspring with a minimized and chemically synthesized genome of $531 \mathrm{kbp}$ and 473 genes. The genome is smaller than that of any autonomously replicating cell. However, it still contains 149 genes of unknown function (Hutchison et al., 2016).

A synthetic minimal genome might also be useful for biotechnological applications. No unknown factors or self-defense mechanisms will lead to complications in the e.g. overproduction of proteins. By the deletion of unnecessary pathways, more energy can be used for the desired product. However, in a real minimal genome, the addition of some functions might be necessary to create a useful strain for biotechnology, since important functions for the production might be deleted. It could be shown, that genome reduction can lead the improved production of recombinant proteins (Morimoto et al., 2008). However, in other cases, disadvantageous changes could be observed, like morphology changes (Hashimoto et al., 2005). 
The model organism B. subtilis was previously used for minimal genome projects. A genome reduction of $25 \%$ was achieved in the MGIM strain, which shows a slightly reduced growth in comparison to the wild type (Ara et al., 2007).

\subsubsection{The MiniBacillus project}

This work is part of the MiniBacillus project. The goal of this project is to construct a B. subtilis strain with a minimal gene set, that is completely understood. Every gene should have an assigned function in the cell. A top-down approach is used, and not necessary parts of the genome are deleted step-by-step. If a growth defect occurs, the strains should be evolved so that arising suppressor mutations can be isolated. These can lead to the restoration of growth and the resulting strain can be used again for new deletions. If a final mini cell is achieved, a transposon mutagenesis can be performed to analyse which genes are still or newly dispensable (Juhas et al., 2014).

The fundament of each minimal genome project is the basic concept, which genes are incorporated into the minimal cell. Therefore, the essential genes need to be first considered. Essential genes cannot be deleted under standard growth conditions. For B. subtilis these growth conditions are LB-glc medium at $37^{\circ} \mathrm{C}$. To identify the essential gene set, a computational or an experimental method can be used (Burgard et al., 2001). In the experimental approach, every single gene is disrupted using different methods, e.g. the integration of a plasmid via single crossover recombination (Kobayashi et al., 2003). The set of essential genes of $B$. subtilis was extensively studied (Juhas et al., 2011; Commichau et al., 2013). An important study in this context was the recently published work of Koo et al. (2017). Two single genes deletion libraries of B. subtilis were created, which comprise the single deletion mutants of each almost 4000 genes. This led to the identification of 257 essential genes. Furthermore, new competence genes were identified, by testing the single deletion strains.

The list of essential genes is not sufficient for the building of a minimal genome. Some functions are essential for $B$. subtilis, but if two genes encode for this function, the single deletion of one is possible and they are therefore not listed as essential genes. In such cases, one of the genes needs to be chosen for the minimal gene set. Furthermore, the competence genes and the genes for genome stability are important to ensure the stability of the strain during the deletion process (Reuß et al., 2016). In some cases, essential genes can be deleted if another gene is already deleted. The antitoxin gene $y x x D$ can only be deleted, if the toxin gene $y x i D$ is already deleted (Holberger et al., 2012).

Starting with this data, a blueprint for a minimal B. subtilis cell was created (Reuß et al., 2016). The conditions for the MiniBacillus are growth at $37^{\circ} \mathrm{C}$ in LB-glc medium. This medium provides many 
nutrients and amino acids. The uptake is favoured instead of the synthesis, since the import needs less genes. This is the case for the amino acids. Mainly the importers remain in the MiniBacillus strain, but some biosynthesis pathways are also listed, since not all amino acid importers are known. The main carbon source of the cell will be glucose. Therefore, the carbon metabolism is reduced to glycolysis and the pentose-phosphate pathway. The citric acid cycle should be completely deleted. Since the LB-glc medium contains not enough nucleotides, that the import would be sufficient to sustain stable growth, nucleotides can still be synthesized in the final MiniBacillus strain. This leads in total to a minimal gene set of 523 protein coding genes, of which 243 are essential and 119 RNA coding genes of which two are essential (Reuß et al., 2016). A scheme of the MiniBacillus cell is shown in Figure 1.

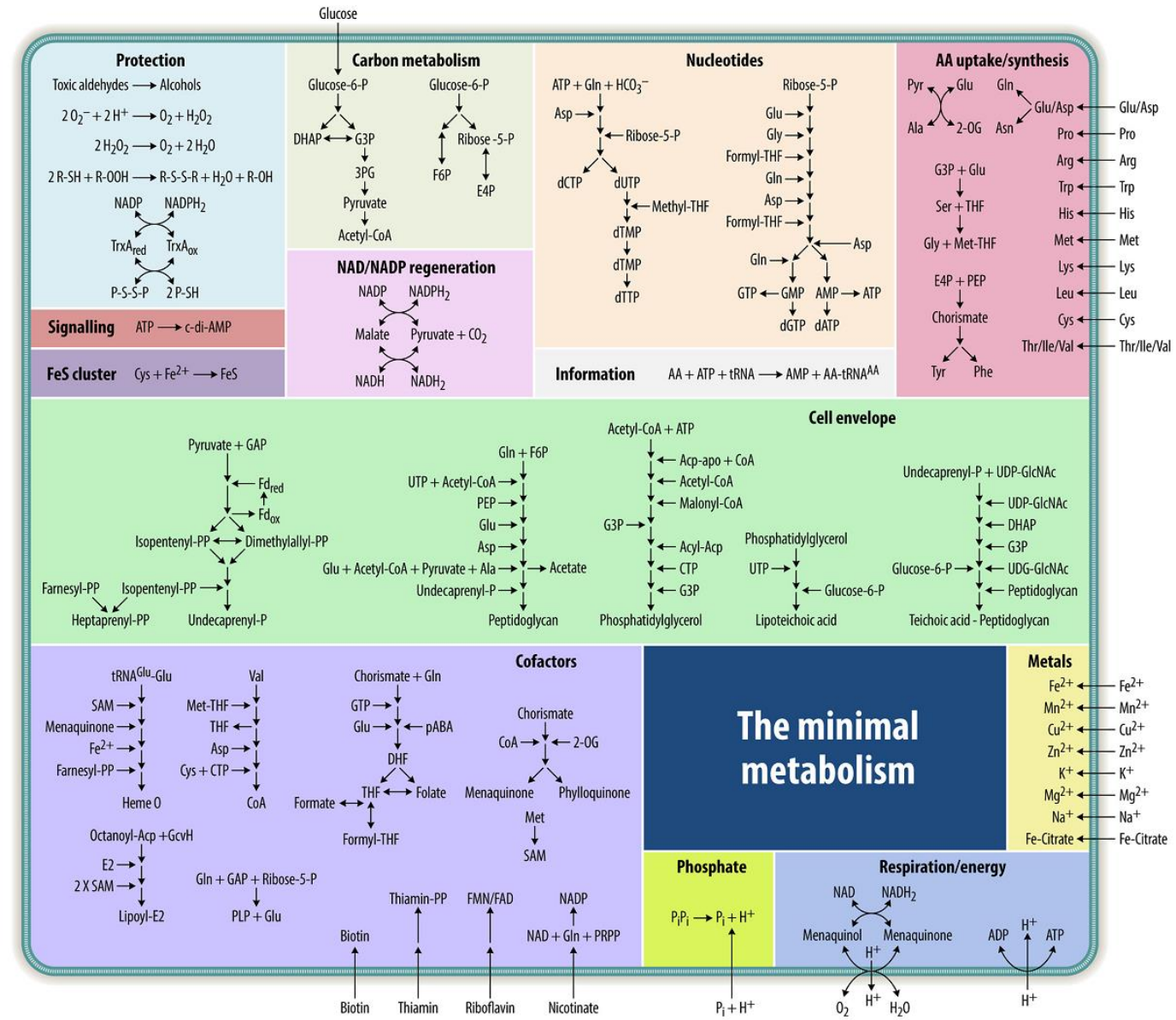

Figure 1: The Blueprint of a MiniBacillus cell. The pathways and importer systems that should retain in the genome minimized B. subtilis cell. E.g. the carbon metabolism is reduced to the gylcolysis and the pentosephosphate pathway (Reuß et al., 2016).

For the creation of the minimal B. subtilis genome, a marker-free deletion system is used. This has the advantage, that no selection marker or scar remains in the genome, which could interfere with the deletion process. The method is based on a selection-counter selection system. A constructed plasmid with the upstream and downstream regions of the desired deletion region 
and a spectinomycin resistance is introduced into the strain. The strain lacks the native phosphotransferase system for the import of mannose, encoded by the genes manA and manP. The introduced plasmid integrates into the chromosome by a single crossover. In a second internal recombination, the cells could, in some cases, lose the deletion region and therefore also the manP gene, that was also encoded on the plasmid (Figure 2). Therefore, the cells will be grown in the presence of mannose. If $\operatorname{man} P$ is still expressed in the cells, mannose-phosphate accumulates to a toxic level and the strain cannot grow. However, the cells that lost the plasmid and the gene are able to grow in the presence of mannose (Wenzel and Altenbuchner, 2015) .

A

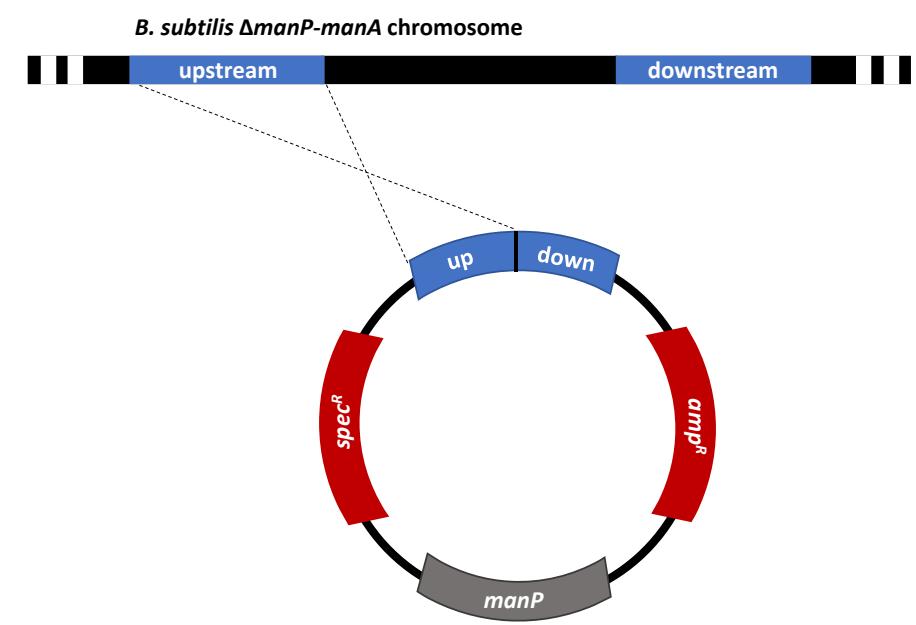

B

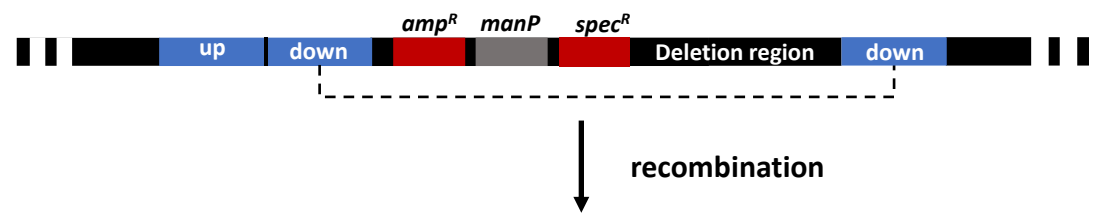

\section{I up | down I |}

Figure 2: The marker-free deletion system of the MiniBacillus project. A The introduction of a constructed plasmid is mediated via a single crossover and the cells are grown in the presence of spectinomycin. $\mathbf{B}$ In a second recombination step, the plasmid is removed from the chromosome, together with the desired deletion region. This deletion needs to be verified via PCR.

The deletion strains also utilize a special competence system, developed by Rahmer et al. (2015), the comKS system. A comKS cassette is introduced into the chromosome with the mannitol inducible promoter of $m t / A$. ComK is the master regulator of competence and if a certain threshold of ComK is reached in the cell, the competence is induced (Maamar and Dubnau, 2005; Smits et al., 2005). With this method, the transformation efficiency is about 7-fold higher in comparison to the competence induction with the amino acid starvation method (Rahmer et al., 2015). Furthermore, the cells can be grown in complex media like LB and not in minimal media that is normally used to induce competence. This is an advantage, especially for the MiniBacillus project, since the 
MiniBacillus strains needs to uptake many compounds, like amino acids, from the complex medium, instead of synthesizing them.

The project was started by the group of Josef Altenbuchner in Stuttgart and the first deletion were performed in the strain $\Delta 6$, in which the main prophages are missing (Wenzel and Altenbuchner, 2015). The strains constructed during the MiniBacillus project are shown in Figure 3. At the point of the deletion strain IIG-Bs27-47-24, with a genome size of $2.83 \mathrm{Mbp}$, a second parallel line was started in Göttingen. Further deletions were performed and the next milestone was achieved with the creation of the MiniBacillus strain PG10. This strain has a genome reduction of $34.54 \%$ in comparison to the wild type strain 168. Interestingly, a multi-omics analysis was performed to compare the strain PG10 with the strain $\Delta 6$. This led to a good overview about the metabolism and expression in PG10. From the transcriptome data, it could be seen that the expression levels of some genes had changed. This information can now be used to adapt the deletion process and counteract occurred problems (Reuß et al., 2017).

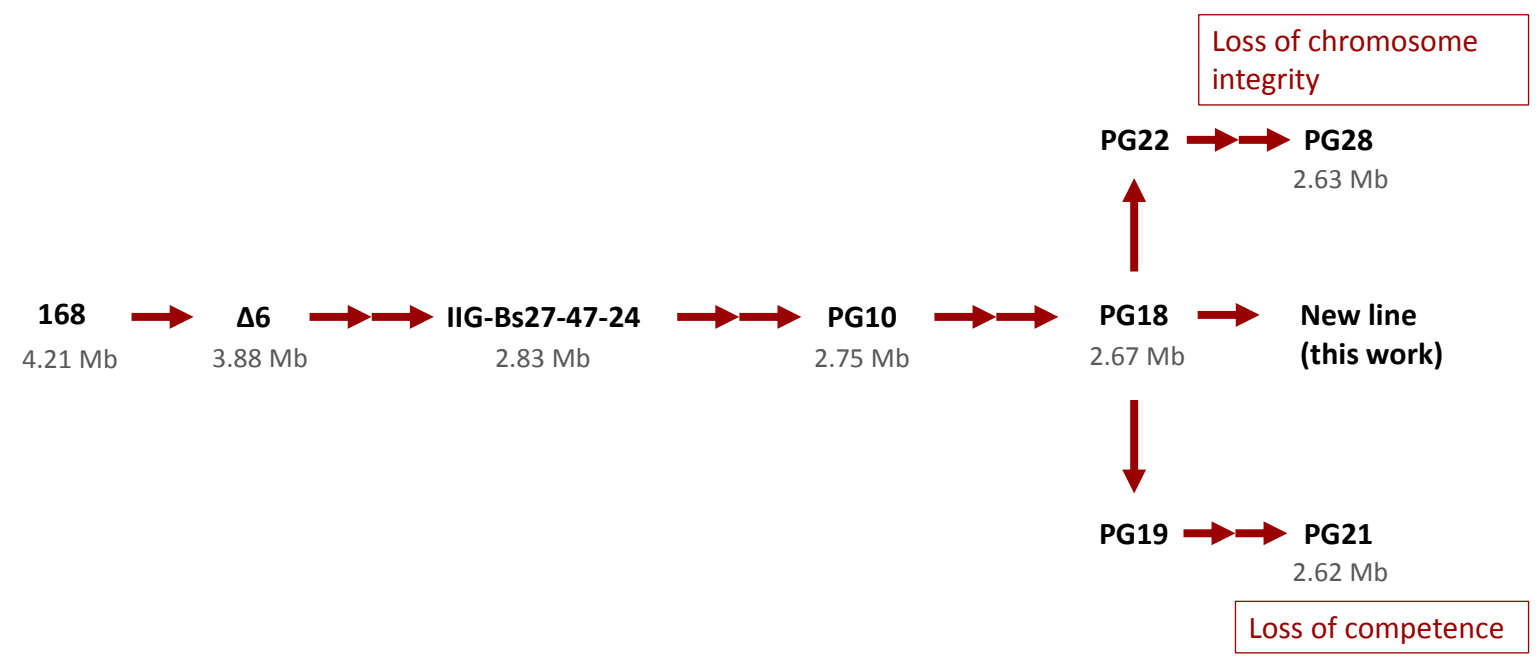

Figure 3: The constructed strains of the MiniBacillus project and the corresponding genome sizes. Some dead end occurred in the deletion process with the strains PG21 and the PG28.

More deletions were performed and the strain PG18 was created, with a genome reduction of $36.61 \%$. This strain shows a good growth under the selected conditions and no problems with competence or genome stability. Several genes were deleted to create the strain PG19, which led to a decreased competence in the following strains PG20 and PG21. This effect was caused by the deletion of $n r n A$, encoding for the nanoRNase NrnA (Reuß, 2017). The deletion of other RNases, e.g. PnpA can also cause a competence reduction (Luttinger et al., 1996). The reduced competence was a dead end for the project and new deletions were again performed based on the stable strain PG18. PG22 was the next constructed strain and its genome was further reduced up to the strain PG28. However, a problem with the genome integrity was recognized. Some deletions could be verified by PCR, but not by whole genome sequencing (WGS). This could already be observed in the 
strain PG22. However, it was discussed if this might be due to a problem with the DNA replication, but the reason for this phenotype is not clear (Reuß, 2017). Therefore, the strain PG18 will be used to create a new line in this work.

It was already mentioned, that a minimal genome strain can be useful for industrial applications. A recent study shows that PG10 is able to produce and secrete proteins that cannot be produced by the wild type strain 168. In this case, four antigens from Staphylococcus aureus were expressed in PG10 (Aguilar Suárez et al., 2019). The reason for the improved production and secretion was seen to be the lack of some proteases in the strain PG10, which could previously been shown to influence the heterologous protein production (Stephenson and Harwood, 1998; Pohl et al., 2013). This shows that genome minimized strains can be useful for the production of proteins in the industry.

\subsection{Central carbon metabolism and the citric acid cycle}

B. subtilis is able to utilize different carbohydrates, like glucose and arabinose, as the sole carbon and energy source (Stülke and Hillen, 2000). Several proteins, like the amylase AmyE, are secreted into the surrounding medium to degrade polysaccharides (Yang et al., 1983). The different sugars are then each imported into the cell and phosphorylated by a specialized phosphotransferase system (PTS) (Saier and Reizer, 1992; Postma et al., 1993). Glucose is the preferred carbon source of $B$. subtilis and the uptake is facilitated by the glucose PTS encoded by ptsGHI (Gonzy-Tréboul et al., 1989; Stülke and Hillen, 2000). However, the metabolism of different carbon and energy sources is highly regulated by carbon catabolite control (Fujita, 2009). Glucose is further metabolized in glycolysis and the pentose-phosphate pathway (Sauer et al., 1997). In glycolysis, glucose-6phosphate is converted in several steps to pyruvate. Furthermore, this pathway provides precursors for the synthesis of amino acids and cell wall components (Ludwig et al., 2001). The genes pdhABCD encode the pyruvate dehydrogenase, which catalyses the reaction of pyruvate to acetyl-coenzyme A (CoA) (Lowe et al., 1983). Acetyl-CoA can further be introduced into the tricarboxylic acid (TCA) cycle or into overflow metabolism by the production of acetate, which is secreted to dispose excess amounts of carbon (Speck and Freese, 1973). The TCA cycle is a central point in the metabolism of many organisms and it has two main functions in the cell. First, the degradation of intermediates to produce energy, and second, the production of important building blocks, e.g. for amino acids (Hanson and Cox, 1967). The TCA cycle of B. subtilis is illustrated in Figure 4. 


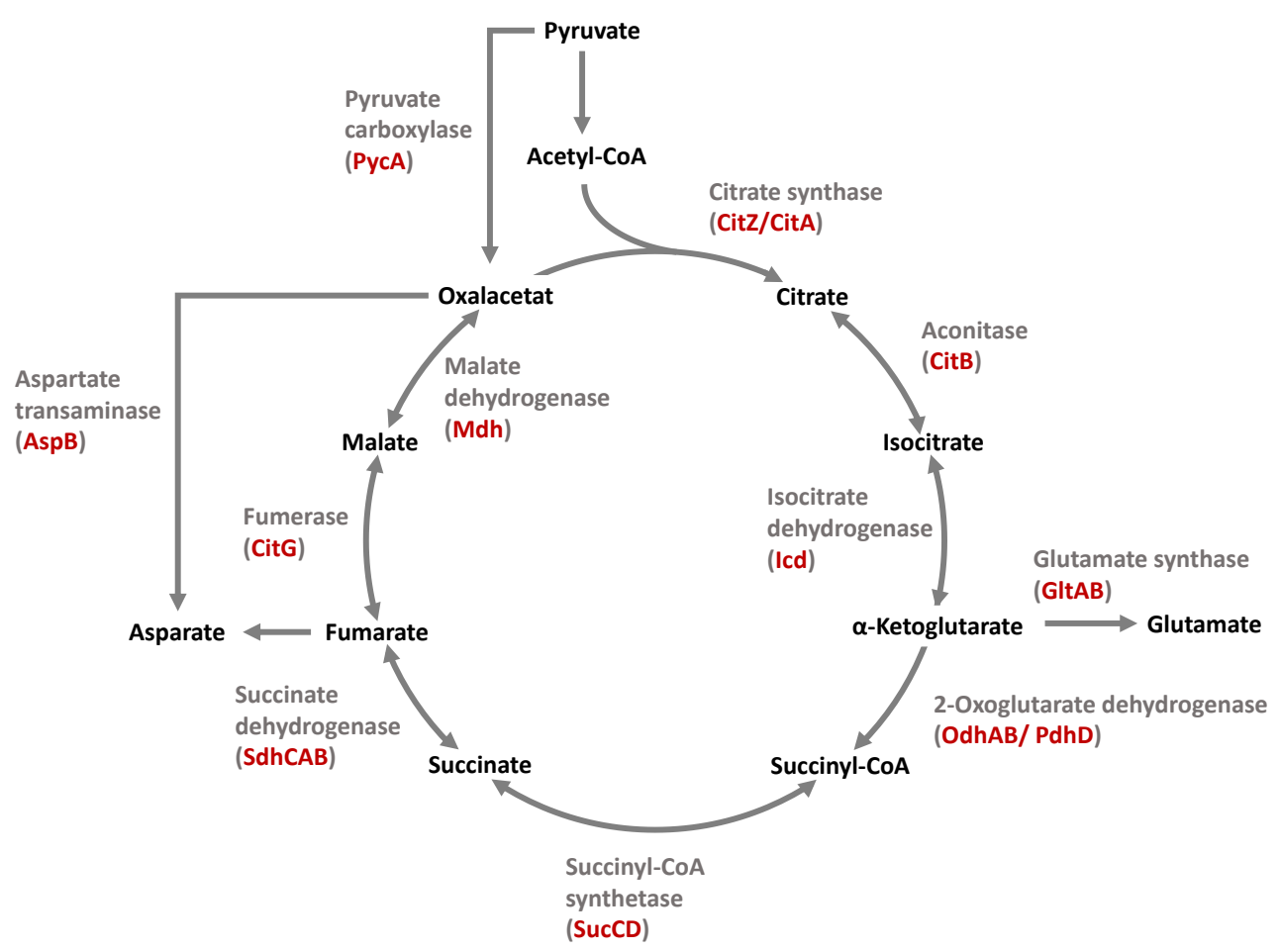

Figure 4: Overview about the TCA cycle in B. subtilis. The connection of the TCA cycle to the nitrogen metabolism is mediated via the synthesis of glutamate from $\alpha$-ketoglutarate. Additionally, oxaloacetate can be converted to aspartate, by the aspartate transaminase AspB.

The first three enzymes of the TCA cycle, the citrate synthase, the aconitase and the isocitrate dehydrogenase have an important role in B. subtilis. Deletion mutants of each of the enzymes are glutamate auxotroph and show defects in sporulation. Acetyl-CoA is introduced into the TCA cycle by the reaction with oxaloacetate to citrate. This reaction is catalysed by the citrate synthase. Interestingly, there are two citrate synthases CitZ and CitA annotated in the B. subtilis genome. CitZ is responsible for the major citrate synthase activity (Jin and Sonenshein, 1994a; Jin and Sonenshein, 1994b). The citrate synthase CitA can compensate the loss of CitZ only partially and it is therefore annotated as a minor citrate synthase (Zschiedrich, 2014). The expression of both enzymes is also differently regulated. CitZ is transcriptionally regulated by $\operatorname{CppA}$ and $\operatorname{CcpC}$ and its expression is therefore repressed by glucose and glutamate (Jourlin-Castelli et al., 2000; Kim et al., 2002). In contrast, the expression of CitA is repressed by glucose, but the combination of glucose with glutamate activates the expression again (Jin and Sonenshein, 1994a). A known but poorly characterized, transcriptional repressor of the citA gene is the LysR-type protein CitR (Jin and Sonenshein, 1994b). However, it is not known, why B. subtilis harbours two citrate synthases. The next step in the TCA cycle is the conversion of citrate to isocitrate, catalysed by the aconitase CitB. Citrate accumulates is a citB deletion mutant and leads to a block in sporulation. This is caused by the formation of chelating complexes of citrate and divalent cations, like $\mathrm{Mn}^{2+}$ and $\mathrm{Fe}^{2+}$, that are necessary to initiate sporulation (Craig et al., 1997). The accumulation of citrate is not only a 
consequence of the missing citrate degradation pathway, the aconitase is also able to bind the citz RNA and destabilize it (Alén and Sonenshein, 1999; Pechter et al., 2013). This enzyme is therefore a trigger enzyme, which can act as an RNA-binding protein in response to the availability of iron, beside its enzymatic activity as an aconitase (Alén and Sonenshein, 1999; Commichau and Stülke, 2008). These two mechanisms ensure the exact regulation of the rate-limiting initial steps of the TCA cycle. Furthermore, the citB deletion mutant or a mutant without enzymatic activity is able to form suppressor mutants, which harbour a mutation in the citrate synthase gene citz. (Pechter et al., 2013). A similar problem of an increasing citrate level and the resulting problem in sporulation can be observed in a deletion mutant of the isocitrate dehydrogenase Icd. This enzyme catalyses the reaction from isocitrate to $\alpha$-ketoglutarate and it is the only enzyme of the TCA cycle, which utilizes NADP as a cofactor (Ramaley and Hudock, 1973). The block in sporulation in an icd deletion mutant, can be reversed by an additional deletion of the citrate synthase CitZ, which leads to the assumption that the level of citrate is here also responsible for the phenotype (Matsuno et al., 1999). C $\mathrm{CpC}$ is the repressor of the citrate synthase CitZ, the aconitase CitB and the isocitrate synthase Icd. Since citZ, icd and $m d h$, the malate dehydrogenase, are encoded in one operon, CcpC binds in the respective promoter region. C $\mathrm{CPC}$ exclusively regulates the expression of TCA cycle genes and responds to the citrate level in the cell. Low citrate levels lead to the repression of citZ and citB, which is again reversed if the citrate concentration increases. In contrast, very high levels of citrate activate the expression of the aconitase by CcpC (Kim et al., 2003b; Kim et al., 2003a).

The TCA cycle intermediate $\alpha$-ketoglutarate is the link to nitrogen metabolism. It can be converted to glutamate by the glutamate synthase GltAB (Wacker et al., 2003). Furthermore, the reaction from glutamate to $\alpha$-ketoglutarate is catalysed by the glutamate dehydrogenases RocG and GudB (Belitsky and Sonenshein, 1998). However, $\alpha$-ketoglutarate can also be converted to succinyl-CoA in the TCA cycle. This reaction is catalysed by the $\alpha$-ketoglutarate dehydrogenase complex encoded by the genes odhAB and pdhD (Carlsson and Hederstedt, 1989). The enzyme PdhD was already mentioned as a part of the pyruvate dehydrogenase complex (Gao et al., 2002). The succinyl-CoA synthetase, encoded by $\operatorname{sucCD}$, catalyses the conversion of succinyl-CoA to succinate (Condon et al., 2002). Succinate is further metabolised to fumarate by the succinate dehydrogenase complex SdhCAB (Melin et al., 1987). SdhC is the membrane bound part of the enzyme and additionally part of the cytochrome b558. The SdhCAB complex is involved in the electron transfer to menaquinone and thereby in the respiratory chain (Hederstedt and Rutberg, 1981; Baureder and Hederstedt, 2011). The next step is catalysed by the fumarase CitG, which is the conversion of fumarate to malate (Miles and Guest, 1985). The fumarase is additionally involved in the DNA damage response, since DNA damage induces the expression of cit $G$ and the protein seems to co- 
localize with the DNA in the cell (Singer et al., 2017). The malate dehydrogenase Mdh catalyses the reaction of malate to oxaloacetate (Jin et al., 1996).

A second, TCA-independent pathway for the production of oxaloacetate exists in B. subtilis. It can be produced from pyruvate by the pyruvate carboxylase PycA. This enzyme is bound to biotin as a cofactor (Henke and Cronan, 2014). This oxaloacetate can be further converted to aspartate. The reaction is catalysed by the aspartate transaminase AspB. Since this is a transaminase reaction, glutamate is needed for this reaction and $\alpha$-ketoglutarate is produced in addition. Aspartate is the precursor of several other amino acids like threonine and isoleucine and a deletion mutant of aspB is auxotrophic for aspartate and asparagine (Dajnowicz et al., 2017; Zhao et al., 2018). Furthermore, aspartate can also be again introduced into the TCA cycle, via the conversion to fumarate. The reaction is catalysed by the L-aspartase AnsB (Sun and Setlow, 1991).

Since the TCA cycle is a major point of the metabolism in B. subtilis, the expression of the TCA cycle genes is tightly regulated. Most of the TCA cycle genes are under control of carbon catabolite repression. The regulator CcpA represses the expression of citZ-icd-mdh, citB, odhAB and $\operatorname{sucCD}$, if enough glucose is available in the cell. Furthermore, the expression of the TCA cycle specific repressor CcpC is also regulated by CcpA (Jin and Sonenshein, 1996; Jourlin-Castelli et al., 2000; Kim et al., 2002). This complex interplay of different regulatory mechanisms emphasizes the important role of the TCA cycle in the cell.

The formation of a metabolome was observed for some enzymes of the TCA cycle. The citrate synthase CitZ, the isocitrate dehydrogenase Icd and the malate dehydrogenase Mdh form the central core of this complex. These proteins are additionally encoded in the same operon. It could be shown that the fumarase CitG and the aconitase CitB can also interact with the malate dehydrogenase (Meyer et al., 2011; Jung and Mack, 2018). This metabolome shows again the important interplay of all TCA cycle enzymes and the important role of the complete TCA cycle as a central metabolic pathway. Furthermore, the TCA cycle seems to be involved in other cellular processes. As mentioned above, sporulation is affected if single TCA cycle genes, especially citZ, citB or $i c d$, are individually deleted.

Although the TCA cycle is a central point in the metabolism of $B$. subtilis, other bacteria like M. pneumoniae have a metabolism without a TCA cycle. The specialized lifestyle is the reason for the reduced metabolism, since all nutrients are provided by the host (Manolukas et al., 1988; Halbedel et al., 2007). Furthermore, also incomplete TCA cycles can be found in Listeria monocytogenes, since the 2-oxoglutarate dehydrogenase, the succinyl-CoA synthetase and the succinate dehydrogenase are absent (Glaser et al., 2001; Kim et al., 2006; Eisenreich et al., 2006). 


\subsection{The amino acid biosynthesis and import of $B$. subtilis}

\subsubsection{The amino acid metabolism of $B$. subtilis}

Amino acids are essential building blocks in the cell. They are important for the synthesis of proteins and they can also be utilized as a source for carbon, energy or nitrogen. Amino acids can be either imported from the medium or synthesised by the cell itself. The central carbon metabolism provides many precursors for amino acid biosynthesis. The different pathways for amino acid synthesis in B. subtilis are shown in Figure 5.

Ribose-5-phosphate can be synthesized in the pentose-phosphate pathway and it can be further converted to the amino acid histidine. Furthermore, glutamate can be produced in the histidine utilization pathway (Wray and Fisher, 1994). Phosphoenolpyruvate (PEP), a glycolytic intermediate, is a compound for the synthesis of aromatic amino acids. In this aromatic acid biosynthesis pathway, chorismate is produced, which is an important intermediate in the cell, since it serves furthermore as a precursor for folate and menaquinone (Driscoll and Taber, 1992; Qin and Taber, 1996; de Saizieu et al., 1997). The aromatic amino acids tryptophan, tyrosine and phenylalanine can be metabolized from chorismate (Nester et al., 1969; Hoch and Nester, 1973).

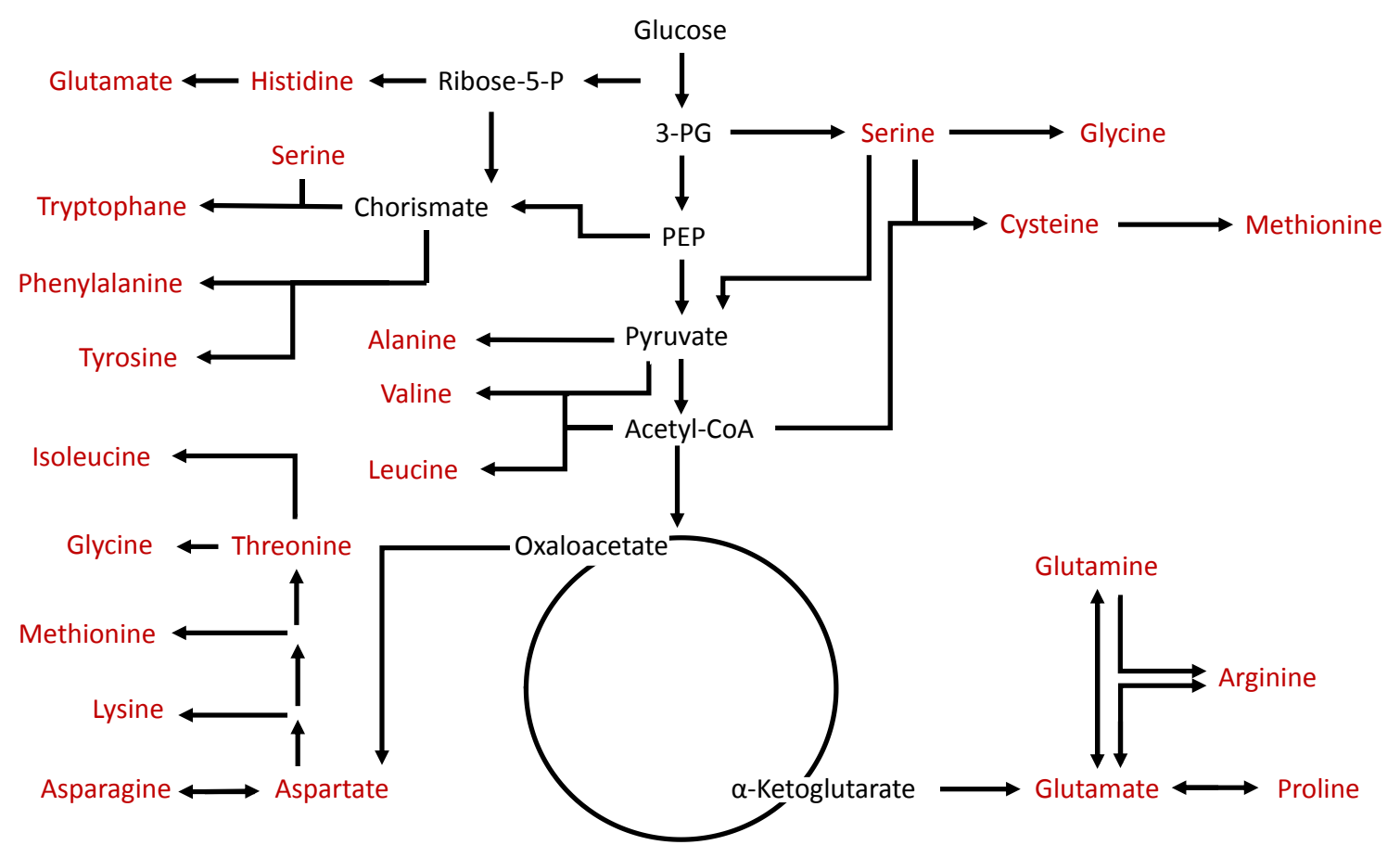

Figure 5: The general pathways of amino acid biosynthesis. Glycolysis provides intermediates like 3phosphoglycerate (3-PG) and phosphoenolpyruvate (PEP) for the biosynthesis of different amino acids. Glutamate and aspartate can be synthesized from intermediates of the TCA cycle and can be used further to synthesize different other amino acids.

The glycolytic product pyruvate also serves as a precursor of for the synthesis of alanine, valine and leucine (Mäder et al., 2004). 3-phosphoglycerate (3-PG) is produced from glucose-6-phosphate and 
can be used to synthesize serine. Since the metabolism of serine is a major research topic of this work, the biosynthesis and utilization pathways are shown in detail in Figure 6.

The phosphoglycerate dehydrogenase SerA catalyses the initial step of the biosynthesis from 3-phosphoglycerate to 3-P-hydroxy-pyruvate. This enzyme is feedback inhibited by serine (Ponce-de-Leon and Pizer, 1972). The intermediate 3-P-hydroxy-pyruvate is further converted to phophoserine by the 3-phosphoserine aminotransferase SerC. Interestingly, the final step in the serine biosynthesis was unknown until YsaA was discovered to be the missing phosphoserine phosphatase. YsaA was therefore named SerB and it catalyses the conversion of phosphoserine to serine (Koo et al., 2017). Serine can be degraded to pyruvate by the L-serine deaminase composed of SdaAA and SdaAB (Chen et al., 2012). Furthermore, glycine can be produced form serine by the serine hydroxymethyltransferase GlyA (Saxild et al., 2001). Acetyl-CoA and serine are precursors to synthesize cysteine, which can furthermore be used to synthesis methionine (Hullo et al., 2007). Interestingly, high serine concentrations can inhibit the growth of $B$. subtilis and become toxic for the cell in minimal medium. The mechanism that is responsible for this effect is unknown, but the addition of some amino acids, like threonine and aspartate, is able to compensate this toxic effect and the cells can grow. Some other amino acids, like arginine and proline, are only able to compensate partially. In contrast to $B$. subtilis, several strains of $B$. thuringiensis are resistant to the growth inhibitory effect of serine (Lachowicz et al., 1996).

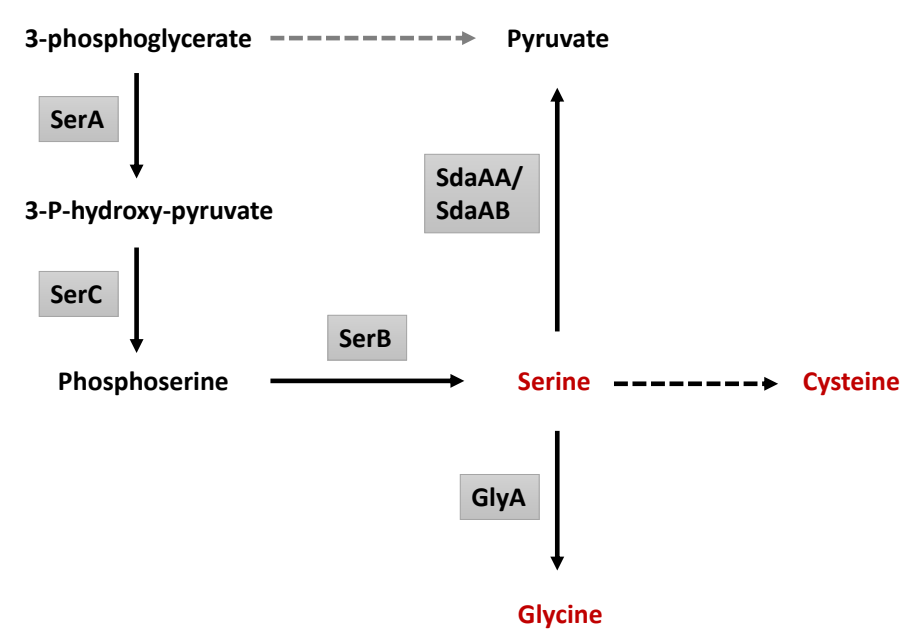

Figure 6: Serine metabolism of B. subtilis. Abbreviations used in this figure: SerA, phosphoglycerate dehydrogenase; SerC: 3-phosphoserine aminotransferase; SerB: phosphoserine phosphatase; SdaAA; Lserine deaminase (alpha chain); SdaAB, L-serine deaminase (beta chain); GlyA, serine hydroxymethyltransferase.

The TCA cycle provides precursors of the amino acid synthesis as well. $\alpha$-ketoglutarate can be converted to glutamate, which serves as an important link between carbon and nitrogen metabolism. Glutamate has an important role in the cell and it was shown to be the most abundant 
metabolite in the E. coli cell (Bennett et al., 2009). In B. subtilis, the amino acid is needed in high concentrations for at least 37 transamination reactions, since the transaminases have a very low affinity for glutamate (Bennett et al., 2009). Interestingly, many of these transamination reactions can be found in the biosynthesis pathways of several amino acids (Oh et al., 2007). As mentioned above, the 3-phosphoserine aminotransferase SerC of the serine biosynthesis pathway catalyses also a transamination reaction. Glutamate serves as the major donor of amino groups and it is a precursor for other amino acids, like glutamine, proline and arginine.

Oxaloacetate, another intermediate of the TCA cycle, can be converted to aspartate. Figure 7 shows the amino acids that can be produced from aspartate. The $B$. subtilis genome encodes three asparagine synthases AsnO, AsnB and AsnH which catalyse the reaction of aspartate to asparagine (Yoshida et al., 1999). Furthermore, aspartate can be converted to L-aspartatyl-4phosphate. This reaction is catalysed by three aspartokinases (DapG, LysC, ThrD), that are each differently regulated by several products of the following pathways, the threonine biosynthesis, the diaminopimelate synthesis and the lysine biosynthesis. DapG, the essential aspartokinase $I$ is feedback inhibited by diaminopimelate, a precursor of the peptidoglycan biosynthesis. Lysine repressed the expression of the aspartokinase II LysC, by a lysine dependent riboswitch. ThrD, the Aspartokinase III, is inhibited by the presence of both threonine and lysine (Graves and Switzer, 1990; Kobashi et al., 2001; Rosenberg et al., 2016).

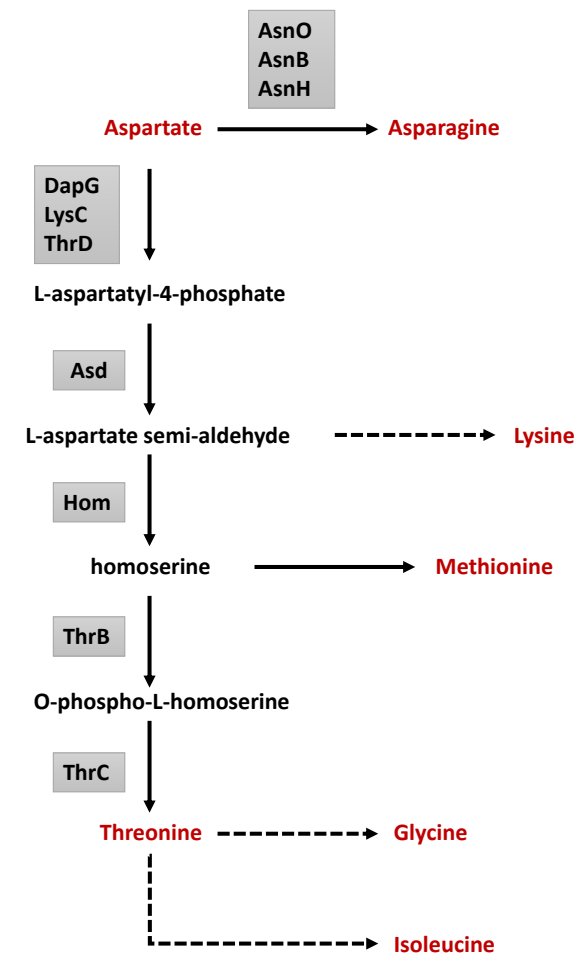

Figure 7: The amino acid biosynthesis pathways derived from aspartate. Abbreviations used in this figure: AsnO, AsnB and AsnH, Asparagine synthases; DapG, aspartokinase I; LysC, aspartokinase II; ThrD, aspartokinase III; Asd, aspartate-semialdehyde dehydrogenase; Hom, homoserine dehydrogenase; ThrB, homoserine kinase; ThrC, threonine synthase. 
L-aspartate semi-aldehyde is produced from L-aspartatyl-4-phosphate by the enzyme aspartatesemialdehyde dehydrogenase Asd. This compound can be further metabolised in the diaminopimelate (DAP) pathway to meso-2,6-diaminopimelate, which is used for either the synthesis of peptidoglycan or for the synthesis of lysine (Rodionov et al., 2003). However, Laspartate semi-aldehyde can also be converted to homoserine by the homoserine dehydrogenase Hom (Parsot and Cohen, 1988). Homoserine can again be used, either for the biosynthesis of methionine or for the conversion to O-phospho-L-homoserine. The last reaction is catalysed by the homoserine kinase. The intermediate O-phospho-L-homoserine is in the next step metabolised to the amino acid threonine (Parsot, 1986). The genes hom, thrC and thrB form an operon and are regulated by several transcription factors. The DNA-binding protein ThrR represses the expression of the hom operon and additionally the expression of the aspartokinase III gene thrD (Rosenberg et al., 2016). Furthermore, the protein CodY is involved in the regulation of the operon. This transcription factor regulates the expression of several nitrogen metabolism genes, competence genes and genes involved in acetate metabolism. The DNA-binding of this global regulator CodY is activated by branched-chain amino acids (BCAA) and GTP. CodY represses the genes required for nutrient-limiting conditions in rich media, since enough BCAAs and GTP stabilize the DNA-binding of CodY. This repression is released, if the BCAA and GTP levels in the cell decrease by a lower availability of nutrients. The hom-thrCB promoter is also repressed by CodY (Fisher, 1999). The expression of the hom operon is also regulated by TnrA (Mirouze et al., 2015). TnrA is a transcription factor, which regulates the expression of several genes under nitrogen limiting conditions (Wray et al., 1996). The amino acid threonine can be further converted to glycine and isoleucine (Mäder et al., 2004). Interestingly, high amounts of threonine in minimal medium inhibit the growth and the formation of spores in B. subtilis. This effect can be compensated by the addition of valine to the medium, which suggests a inhibition of valine biosynthesis by threonine (Lamb and Bott, 1979a). Similarly, inhibitory effects of high isoleucine or valine concentrations could be observed (Teas, 1950; Lachowicz et al., 1996). Therefore, the level of some amino acids could have a regulatory function in the cell.

\subsubsection{Amino acid transporters}

B. subtilis transports different kinds of substrates. This task is fulfilled by different transporter proteins. In general, the proteins can be divided according to the energy source, into channels, primary transporters and secondary transporters. Channels transport substrates by an energy independent concentration gradient. Primary transporters use the energy from the hydrolysis of ATP for the active transport. Furthermore, secondary transporters are dependent on the ion 
gradient as a transport energy source (Saier, 2000). Transporters import and export different compounds, like vitamins, sugars and amino acids. A very important class of primary transporters are the ATP-binding cassette $(A B C)$ transporters. These can be divided into the importers, the exporters and the proteins that are not involved in transport, but in the regulation of processes like DNA repair (Higgins et al., 1986; Davidson et al., 2008). The $A B C$ transporter consist of three domains: the nucleotide-binding domain, the membrane spanning domain and the solute-binding domain (Quentin et al., 1999). The arginine importer encoded by artPQR is an example for an ABC transporter in B. subtilis (Yu et al., 2015).

The secondary transporters are the largest superfamily of amino acid transporters (Saier, Jr, 2000). An important group within the family is the amino acid-polyamine-organocation (APC) superfamily, which includes solute:cation symporters and solute:solute antiporters (Jack et al., 2000). Many amino acid transporters of $B$. subtilis can be classified in this group. The proline and gamma-amino butyric acid permease GabP and the branched-chain amino acid and threonine transporter BcaP are examples for transporters of the APC family (Zaprasis et al., 2014; Belitsky, 2015). Symporters of the secondary transporters are divided by the ions that are similarly taken up with the solute. Members of the solute:sodium symporter (SSS) family, like the proline importer PutP from B. subtilis, import sodium ions together with the desired compound (Moses et al., 2012). Although, transporters can be divided into different classes, some are not limited to certain substrates and they are rather unspecific. Similar amino acids can often be imported by the same transporter. The importer BcaP from B. subtilis can import valine and isoleucine, since both are branched-chain amino acids (BCAA). Additionally, the threonine transport of BcaP was observed (Belitsky, 2015). Since the addition of serine, alanine and asparagine can decrease the uptake of isoleucine, BcaP might also be involved in the transport of these amino acids (Belitsky, 2015). The uptake of an amino acid is in most cases not facilitated by only one transporter. Although, BcaP seems to facilitate the major uptake of valine and isoleucine, two additional importers are known, $\mathrm{BraB}$ and $\mathrm{BrnQ}$. The reason for the different uptake systems is that each system is active under different conditions. All three genes $b c a P, b r n Q$ and $b r a B$ are repressed via CodY under high BCAA concentrations. However, BraB is active at intermediate CodY levels, since it is additionally repressed by ScoC, a transition state regulator. ScoC represses the expression of BraB only if CodY is present in low concentrations, since CodY inhibits the expression of ScoC (Belitsky et al., 2015). This ensures a certain level of BCAA import, although the expression of the other transporters are still repressed. BrnQ in contrast is overall very low expressed, since the AzlB protein negatively regulates the BrnQ repression, but the reason for this is not completely understood. The three 
different uptake systems for valine and isoleucine are therefore tightly regulated to ensure the adapted uptake of these amino acids to the current cell status (Belitsky, 2015).

Furthermore, some transporters are only expressed and active during low substrate conditions. These are high-affinity transporters and they exhibit a high affinity to the substrate (Bosdriesz et al., 2018). All three valine and isoleucine transporters BcaP, BraB, BrnQ are highaffinity transporters and are active during low BCAA concentrations, since the amount of the repressor CodY is also low. A triple deletion mutant of the transporters, combined with a BCAA auxotrophic strain, is still able to grow on high BCAA concentrations. Therefore, at least one lowaffinity system must be encoded in the $B$. subtilis genome. Low-affinity transporters are expressed at high substrate conditions (Belitsky, 2015; Bosdriesz et al., 2018).

Although, many amino acid transporters of $B$. subtilis are known and they can be divided into different group of transporters, the import of some amino acids is poorly characterized. For the amino acids phenylalanine and tyrosine, no importers are annotated at all. For the serine import only the serine/threonine exchanger SteT is described. SteT is similar to known amino acid transporters from humans and it exhibit a serine transport function in proteoliposomes (Reig et al., 2007). Since, high serine concentrations can inhibit the cell growth, the uptake of serine is most likely higher regulated and different uptake systems could be involved. Furthermore, there are some membrane proteins annotated, which show similarities to known amino acid transporters, but are of unknown function.

\subsection{Aim of this work}

This work focuses on the MiniBacillus project. The first goal is to reduce the genome of the MiniBacillus strain PG18 further and to analyse the impact of the performed deletion on the strain. Data from multi-omics experiments, obtained in previous works, will be utilized to adapt the deletion process and improve the strain. Furthermore, the strain will be phenotypically analysed and compared on a genome and proteome level. Since the MiniBacillus project is mainly about understanding life, the second objective of this work is to analyse the function of the TCA cycle in the cell. In the final minimal cell, the TCA cycle will be not necessary. Therefore, it needs to be analysed, if a deletion of the TCA cycle strain is possible in a wild type strain and if the deletion is a disadvantage for the cell. Not all amino acid importers are known for B. subtilis. However, this knowledge would be an advantage for the MiniBacillus project, since the mini cell could import all necessary amino acids from the complex medium. Therefore, the transporters of unknown function should be characterized further, especially for the amino acid serine. Furthermore, the mechanism 
and physiological role of the serine toxicity should be further analysed. These results will all contribute to the progress of the MiniBacillus project. 


\section{Materials and Methods}

\subsection{Materials}

Materials, chemicals, equipment, commercial systems, enzymes and oligonucleotides are listed in the appendix.

\subsubsection{Bacterial strains and plasmids}

Bacterial strains and plasmids are listed in the appendix.

\subsubsection{Growth media}

Buffers, solutions and media were prepared with deionized water and autoclaved for $20 \mathrm{~min}$ at $121^{\circ} \mathrm{C}$ and 2 bar, unless otherwise stated. Thermolabile substances were dissolved and sterilized by filtration.

\section{Bacterial growth media}

E. coli was grown in LB and M9 medium, whereas B. subtilis was grown in LB, SP and C-minimal media, supplemented with additives as indicated. For solidification, basic media were supplemented with 1.5\% (w/v) agar (Blötz et al., 2017).

\section{Complex media}

LB medium

(1 I)

SP medium

(1 l)

Starch medium

(1 I)

$10 \mathrm{~g}$ Tryptone

$5 \mathrm{~g} \quad$ Yeast extract

$10 \mathrm{~g} \mathrm{NaCl}$

$8 \mathrm{~g} \quad$ Nutrient Broth

$0.25 \mathrm{~g}_{\mathrm{MgSO}_{4}} \times 7 \mathrm{H}_{2} \mathrm{O}$

$1 \mathrm{~g} \quad \mathrm{KCl}$

Solve and autoclave, after cooling down addition of:

$1 \mathrm{ml} \quad \mathrm{CaCl}_{2}(0,5 \mathrm{M})$

$1 \mathrm{ml} \quad \mathrm{MnCl}_{2}(10 \mathrm{mM})$

$2 \mathrm{ml}$ Ammonium iron citrate $(2.2 \mathrm{mg} / \mathrm{ml})$ 


\section{Minimal media}

10x MN medium

(1 I)

MNGE medium

$(10 \mathrm{ml})$

C minimal medium

$(100 \mathrm{ml})$

CE medium

$(100 \mathrm{ml})$

CSE medium

$(100 \mathrm{ml})$
$136 \mathrm{~g} \quad \mathrm{~K}_{2} \mathrm{HPO}_{4} \times 3 \mathrm{H}_{2} \mathrm{O}$

$60 \mathrm{~g} \quad \mathrm{KH}_{2} \mathrm{PO}_{4}$

$10 \mathrm{~g}$ Sodium citrate $\times 2 \mathrm{H}_{2} \mathrm{O}$

$1 \mathrm{ml} \quad 10 \times \mathrm{MN}$ medium

$1 \mathrm{ml} \quad$ Glucose (20\%)

$50 \mu \mathrm{l} \quad$ Potassium glutamate (40\%)

$50 \mu \mathrm{l} \quad$ Ammonium iron citrate $(2.2 \mathrm{mg} / \mathrm{ml})$

$100 \mu \mathrm{l}$ Tryptophan $(5 \mathrm{mg} / \mathrm{ml})$

$30 \mu \mathrm{l} \quad \mathrm{MgSO} 4 \times 7 \mathrm{H} 2 \mathrm{O}(1 \mathrm{M})$

$+/-100 \mu \mathrm{l}$ CAA (10\%)

$20 \mathrm{ml} 5 \times \mathrm{C}$-salts

$1 \mathrm{ml}$ Tryptophan $(5 \mathrm{mg} / \mathrm{ml})$

$1 \mathrm{ml}$ Ammonium iron citrate $(2.2 \mathrm{mg} / \mathrm{ml})$

$1 \mathrm{ml} \quad$ III' salts

$20 \mathrm{ml} 5 \times \mathrm{C}$-salts

$1 \mathrm{ml}$ Tryptophan $(5 \mathrm{mg} / \mathrm{ml})$

$1 \mathrm{ml}$ Ammonium iron citrate $(2.2 \mathrm{mg} / \mathrm{ml})$

$1 \mathrm{ml} \quad$ III' salts

$2 \mathrm{ml}$ Potassium glutamate (40\%)

$20 \mathrm{ml} 5 \times \mathrm{C}$-salts

$1 \mathrm{ml}$ Tryptophan $(5 \mathrm{mg} / \mathrm{ml})$

$1 \mathrm{ml}$ Ammonium iron citrate $(2.2 \mathrm{mg} / \mathrm{ml})$

$1 \mathrm{ml} \quad$ III' salts

$2 \mathrm{ml}$ Potassium glutamate (40\%)

$2 \mathrm{ml}$ Sodium Succinate (30\%) 


$\begin{array}{lll}\text { M9 (-CAA) medium } & 50 \mathrm{ml} & 20 \times \mathrm{M} \text { base medium } \\ (100 \mathrm{ml}) & 1 \mathrm{ml} & 1 \mathrm{M} \mathrm{MgSO}_{4} \\ 1 \mathrm{ml} & 0.1 \mathrm{M} \mathrm{CaCl}_{2} \\ 0.5 \mathrm{ml} & 1 \mathrm{mM} \mathrm{FeCl}_{3} \\ 1 \mathrm{ml} & \text { Glucose }(50 \%) \\ 1 \mathrm{ml} & \text { Thiamine- } \mathrm{Cl}_{2}(1 \mathrm{mg} / \mathrm{ml})\end{array}$

\section{Solutions and additives}

\begin{tabular}{|c|c|}
\hline $5 \times$ C-salts & $\begin{array}{ll}20 \mathrm{~g} & \mathrm{KH}_{2} \mathrm{PO}_{4}\end{array}$ \\
\hline \multirow[t]{2}{*}{ (1 I) } & $80 \mathrm{~g} \quad \mathrm{~K}_{2} \mathrm{HPO}_{4} \times 3 \mathrm{H}_{2} \mathrm{O}$ \\
\hline & $16.5 \mathrm{~g} \quad\left(\mathrm{NH}_{4}\right)_{2} \mathrm{SO}_{4}$ \\
\hline III' salts & $0.232 \mathrm{~g} \mathrm{MnSO}_{4} \times 3 \mathrm{H}_{2} \mathrm{O}$ \\
\hline (1 I) & $12.3 \mathrm{~g} \quad \mathrm{MgSO}_{4} \times 7 \mathrm{H}_{2} \mathrm{O}$ \\
\hline 20x M9 base & $140 \mathrm{~g} \quad \mathrm{Na}_{2} \mathrm{HPO}_{4} \times 2 \mathrm{H}_{2} \mathrm{O}$ \\
\hline \multirow[t]{3}{*}{ (1 I) } & $\mathrm{KH}_{2} \mathrm{PO}_{4}$ \\
\hline & $\mathrm{NH}_{4} \mathrm{Cl}$ \\
\hline & Ad to $1000 \mathrm{ml}$ with deionized water \\
\hline
\end{tabular}

\section{Antibiotics}

Antibiotics were prepared as 1000 -fold concentrated stock solutions. Kanamycin, spectinomycin, lincomycin, zeocin and ampicillin were dissolved in deionized water, chloramphenicol, erythromycin and tetracycline in $70 \%$ ethanol. All solutions were filtrated and stored at $-20^{\circ} \mathrm{C}$. For the selection of ermC, erythromycin and lincomycin were used in combination.

\section{Selection concentration for $E$. coli}

Ampicillin

$100 \mu \mathrm{g} / \mathrm{ml}$

Kanamycin

$50 \mu \mathrm{g} / \mathrm{ml}$ 


\section{Selection concentration for B. subtilis}

$\begin{array}{ll}\text { Kanamycin } & 10 \mu \mathrm{g} / \mathrm{ml} \\ \text { Spectinomycin } & 150 \mu \mathrm{g} / \mathrm{ml} \\ \text { Lincomycin } & 25 \mu \mathrm{g} / \mathrm{ml} \\ \text { Zeocin } & 35 \mu \mathrm{g} / \mathrm{ml} \\ \text { Chloramphenicol } & 5 \mu \mathrm{g} / \mathrm{ml} \\ \text { Erythromycin } & 2 \mu \mathrm{g} / \mathrm{ml} \\ \text { Tetracycline } & 12.5 \mu \mathrm{g} / \mathrm{ml}\end{array}$

2.2. Methods

\subsubsection{General methods}

Some general methods used in this work are listed in Table 1 with the corresponding literature.

Table 1: General methods

\begin{tabular}{ll}
\hline Method & Reference \\
\hline Absorption measurement & Sambrook et al., 1989 \\
Precipitation of nucleic acids & Sambrook et al., 1989 \\
Gel electrophoresis of DNA & Sambrook et al., 1989 \\
Plasmid preparation from E. coli & Sambrook et al., 1989 \\
Ligation of DNA fragments & Sambrook et al., 1989 \\
Determination of protein amounts & Bradford, 1976 \\
Gel electrophoresis of proteins (denaturing) & Laemmli, 1970 \\
Sequencing according to the chain termination method & Sanger et al., 1992 \\
\hline
\end{tabular}

\subsubsection{Cultivation of bacteria}

Unless otherwise stated, E. coli was grown in LB or M9 -CAA medium at $37^{\circ} \mathrm{C}$ and $200 \mathrm{rpm}$ in tubes and flasks. B. subtilis was grown in LB, SP, C-Glc, CE-Glc, CSE-Glc or MNGE medium at $37^{\circ} \mathrm{C}$ or $28^{\circ} \mathrm{C}$ in tubes and flasks. Fresh colonies from plates or $-80^{\circ} \mathrm{C}$ cryo cultures supplemented with $10 \%$ DMSO were used for inoculation. The growth was measured as the optical density at $600 \mathrm{~nm}$. 


\subsubsection{Storage of bacteria}

E. coli was kept on LB medium agar plates up to 4 weeks at $4^{\circ} \mathrm{C}$. DMSO cultures were used for longterm storage. B. subtilis was cultured on SP or LB plates and stored in DMSO stocks. For a DMSO stock, $900 \mu \mathrm{l}$ of a fresh overnight culture was mixed with $100 \mu \mathrm{l}$ of DMSO. Stocks were snap frozen and stored at $-80^{\circ} \mathrm{C}$. SP agar tubes were used for the long-term storage of B. subtilis (Blötz et al., 2017).

\subsubsection{Preparation of competent E. coli and transformation}

\section{Preparation of competent cells in SOB medium}

A culture of $E$. coli DH5 $\alpha$ or XL1blue cells were used to inoculate $250 \mathrm{ml} \mathrm{SOB}$-medium over night at $18^{\circ} \mathrm{C}$. After reaching an $\mathrm{OD}_{600}$ of $0.5-0.9$ the culture was cooled down by incubation for $10 \mathrm{~min}$ on ice. The cells were harvested by centrifugation $\left(10 \mathrm{~min} ; 4000 \mathrm{rpm} ; 4^{\circ} \mathrm{C}\right.$ ) and resuspended in $80 \mathrm{ml}$ of ice-cold TB-Buffer. A final concentration of 7\% DMSO was added and aliquots of $200 \mu \mathrm{l}$ were frozen in liquid nitrogen and stored at $-80^{\circ} \mathrm{C}$ (Blötz et al., 2017).

$\begin{array}{lll}\text { SOB-Medium } & 20 \mathrm{~g} & \text { Tryptone } \\ \text { (1 I) } & 5 \mathrm{~g} & \text { yeast extract } \\ & 0.584 \mathrm{~g} & \mathrm{NaCl} \\ & 0.188 \mathrm{~g} & \mathrm{KCl} \\ & \text { Ad. } 1 \mathrm{I} & \text { deion. } \mathrm{H}_{2} \mathrm{O} \\ & & \\ & 1.51 \mathrm{~g} & \mathrm{PIPES} \\ \text { TB-Buffer } & 1.1 \mathrm{~g} & \mathrm{CaCl}_{2} \times \mathrm{H}_{2} \mathrm{O} \\ \text { (500 ml, pH 6.7) } & 9.32 \mathrm{~g} & \mathrm{KCl} \\ & \mathrm{Ad} 472.5 \mathrm{ml} & \text { deion. } \mathrm{H}_{2} \mathrm{O} \\ & 27.5 \mathrm{ml} & \mathrm{MnCl}_{2}(1 \mathrm{M})\end{array}$

\section{Preparation of competent cells in $\mathrm{CaCl}_{2}$ medium}

An overnight culture of E. coli BL21 or JM109 was used to inoculate $100 \mathrm{ml}$ of LB medium. This culture was grown to an $\mathrm{OD}_{600}$ of 0.3 and $10 \mathrm{ml}$ of cells were harvested for $6 \mathrm{~min}$ at $5000 \mathrm{rpm}$ and $4^{\circ} \mathrm{C}$. The pellet was resuspended in $5 \mathrm{ml}$ of a $50 \mathrm{mM} \mathrm{CaCl}_{2}$ solution. The cells were incubated on ice for $30 \mathrm{~min}$ and again centrifuged as described before. $1 \mathrm{ml}$ of a $50 \mathrm{mM} \mathrm{CaCl}$ solution was used to resuspend the cells, which were then used for the transformation method (Blötz et al., 2017). 


\section{Transformation of competent $E$. coli cells}

$200 \mu \mathrm{l}$ competent cells were defrosted on ice and mixed with 10-100 ng DNA. After inoculation for $30 \mathrm{~min}$ on ice, the heat shock was performed at $42^{\circ} \mathrm{C}$ for 90 seconds. $500 \mu \mathrm{l}$ of LB medium was added and the samples were incubated for $60 \mathrm{~min}$ at $37^{\circ} \mathrm{C}$ at $200 \mathrm{rpm} .150 \mu \mathrm{l}$ and the rest of the solution were plated on LB selection plates (Blötz et al., 2017).

\subsubsection{Preparation of competent $B$. subtilis cells and transformation}

\section{Preparation of competent cells in MNGE medium and transformation}

An overnight culture of $B$. subtilis was used to inoculate $10 \mathrm{ml}$ of MNGE medium containing $1 \%$ CAA to an optical density of 0.1 . The culture was grown at $37^{\circ} \mathrm{C}$ at $200 \mathrm{rpm}$ until an $\mathrm{OD}_{600}$ of 1.3 was reached. Then the culture was diluted with $10 \mathrm{mI}$ MNGE medium without CAA and incubated again for one hour. After this incubation step, $400 \mu \mathrm{l}$ of competent cells were incubated with 0.1-1 $\mu \mathrm{g}$ DNA for $30 \mathrm{~min}$ at $37^{\circ} \mathrm{C}$ with at $200 \mathrm{rpm}$. Afterwards, $100 \mu \mathrm{l}$ of expression mix were added and the samples were incubated at $37^{\circ} \mathrm{C}$ for one hour. The cell suspension was spread onto SP or LB selection plate (Blötz et al., 2017).

$\begin{array}{lll}\text { Expression mix }(1.05 \mathrm{ml}) & 500 \mu \mathrm{l} & \text { yeast extract } \\ 250 \mu \mathrm{l} & \text { CAA (10\%) } \\ 250 \mu \mathrm{l} & \text { deion. } \mathrm{H}_{2} \mathrm{O} \\ 50 \mu \mathrm{l} & \text { Tryptophan }(5 \mathrm{mg} / \mathrm{ml})\end{array}$

\section{Preparation of competent cells with the mannitol-inducible comKS system}

$500 \mu \mathrm{l}$ of an overnight culture is used to inoculate $5 \mathrm{ml}$ of LB medium in a baffled flask, that is incubated for $90 \mathrm{~min}$ at $37^{\circ} \mathrm{C}$ and $200 \mathrm{rpm}$. To induce the competence, $5 \mathrm{ml} \mathrm{LB}$ with $0.5 \%$ mannitol and $5 \mathrm{mM} \mathrm{MgCl} 2$ are added to the culture. After another incubation step of $90 \mathrm{~min}$ at $37^{\circ} \mathrm{C}$ and 200 $\mathrm{rpm}$, the cells are harvested by centrifugation for $10 \mathrm{~min}$ at $4500 \mathrm{rpm}$. The cells are re-suspended in $10 \mathrm{ml}$ LB medium and $1 \mathrm{ml}$ of the suspension is mixed with 0.1-1 $\mu \mathrm{g}$ DNA. The samples are incubated for $60 \mathrm{~min}$ at $37^{\circ} \mathrm{C}$ and $200 \mathrm{rpm} .150 \mu \mathrm{l}$ and the rest of the solution were plated on LB selection plates (Rahmer et al., 2015; Blötz et al., 2017). 


\subsubsection{Preparation and detection of DNA}

\section{Isolation of genomic DNA of $B$. subtilis}

For the isolation of $B$. subtilis gDNA, the cells of a $4 \mathrm{ml}$ LB overnight culture were harvested. The gDNA was extracted, using the peqGOLD Bacterial DNA Kit from PEQLAB. Deionized water was used for elution of the DNA from the columns (Blötz et al., 2017).

\section{Isolation of plasmid DNA from E. coli}

Plasmid DNA was extracted from E. coli cultures, using the NucleoSpin ${ }^{\circledR}$ Plasmid Kit from MacheryNagel. Deionized water was used for the elution of the plasmids from the columns (Blötz et al., 2017).

\section{Purification of DNA}

The QIAquick PCR purification Kit was used for the purification of DNA fragments. For the elution of the DNA from the column, deionized water was used (Blötz et al., 2017).

\section{Agarose gel electrophoresis}

Agarose gel electrophoresis was used to separate DNA fragments by size and thereby analyse the respective size. $1 \%$ agarose gels $(\mathrm{w} / \mathrm{v})$ were prepared in TAE Buffer together with HDGreen ${ }^{\mathrm{TM}}$ DNADye from Intas. The DNA samples were supplemented with 5x DNA loading dye and loaded onto the gel, together with an EcoRI/ HindIII digested $\lambda$-DNA marker to estimate the size of the DNA fragments. A voltage of $140 \mathrm{~V}$ was applied until the colour marker reached the last third of the gel. For the detection of the DNA the GelDoc ${ }^{\mathrm{TM}}$ from Biorad was used by excitation with UV light (254 nm) (Blötz et al., 2017).

50x TAE Buffer

5x DNA loading dye

$$
\begin{array}{ll}
242 \mathrm{~g} & \text { Tris-base } \\
57.1 \mathrm{ml} & \text { Acetic acid (100\%) } \\
100 \mathrm{ml} & \text { EDTA }(0.5 \mathrm{M}, \mathrm{pH} 8.0)
\end{array}
$$

Ad to $1000 \mathrm{ml}$ with deionized water

$\begin{array}{ll}5 \mathrm{ml} & \text { Glycerol (100\%) } \\ 200 \mu \mathrm{l} & 50 x \mathrm{TAE} \\ 10 \mathrm{mg} & \text { Bromphenol blue } \\ 10 \mathrm{mg} & \text { Xylene cyanol } \\ 4.8 \mathrm{ml} & \mathrm{dH}_{2} \mathrm{O}\end{array}$




\section{Sequencing of DNA}

Sequencing of plasmids and DNA fragments was performed by Microsynth AG with the chain termination method. Whole genome sequencing was carried out by the Göttingen Genomic Laboratory.

\section{Cloning procedure}

DNA restriction enzymes from ThermoFisher were used to cleave the PCR fragment and the respective plasmid. The manufacturer's instructions for enzyme concentration, incubation conditions and heat inactivation after the reaction were followed. The vector was dephosphorylated by the addition of $1 \mu \mathrm{l}$ alkaline phosphatase (ThermoFisher) and incubation at $37^{\circ} \mathrm{C}$ for $15 \mathrm{~min}$. Afterwards the vector and the insert were ligated using T4-DNA ligase (ThermoFisher). The ligation reaction contained $150 \mathrm{ng}$ of vector DNA and a 5-fold excess of insert DNA and was incubated $2 \mathrm{~h}$ at RT or overnight at $16^{\circ} \mathrm{C}$ (Blötz et al., 2017).

\section{Polymerase chain reaction (PCR)}

The PCR reaction was performed with genomic or plasmid DNA as template in a total volume of 50 $\mu \mathrm{l}$ with Phusion ${ }^{\mathrm{TM}}$ polymerase or DreamTaq polymerase.

Reaction mix for Phusion ${ }^{\mathrm{TM}}$ polymerase $(50 \mu \mathrm{l})$ :

$\begin{array}{ll}10 \mu \mathrm{l} & 5 \times \mathrm{HF} \text { reaction buffer } \\ 2 \mu \mathrm{l} & \text { dNTPs }(12.5 \mu \mathrm{mol} / \mathrm{ml}) \\ 2 \mu \mathrm{l} & \text { forward primer }(20 \mathrm{pmol}) \\ 2 \mu \mathrm{l} & \text { reverse primer }(20 \mathrm{pmol}) \\ 1 \mu \mathrm{l} & \text { template DNA }(1 \mathrm{ng} / \mu \mathrm{l}) \\ 0.2 \mu \mathrm{l} & \text { Phusion }^{\text {TM }} \text { polymerase }(2 \mathrm{U} / \mu \mathrm{l}) \\ 32.8 \mu \mathrm{l} & \mathrm{dH}_{2} \mathrm{O}\end{array}$

Reaction mix for DreamTaq (50 $\mu$ l):

$\begin{array}{ll}5 \mu \mathrm{l} & 10 x \text { DreamTaq reaction buffer } \\ 2 \mu \mathrm{l} & \text { dNTPs }(12.5 \mu \mathrm{mol} / \mathrm{ml}) \\ 2 \mu \mathrm{l} & \text { forward primer }(20 \mathrm{pmol}) \\ 2 \mu \mathrm{l} & \text { reverse primer }(20 \mathrm{pmol}) \\ 1 \mu \mathrm{l} & \text { template DNA }(1 \mathrm{ng} / \mu \mathrm{l}) \\ 0.25 \mu \mathrm{l} & \text { DreamTaq polymerase }(2 \mathrm{U} / \mu \mathrm{l}) \\ 37.75 \mu \mathrm{l} & \mathrm{dH}_{2} \mathrm{O}\end{array}$


The samples were briefly vortexed, centrifuged and the reaction was placed into the Themocycler with the following programs:

\section{Standard program for the Phusion ${ }^{\mathrm{TM}}$ polymerase}

\begin{tabular}{lll|l}
\hline Initial denaturation & $98^{\circ} \mathrm{C}$ & $5 \mathrm{~min}$ & \\
Denaturation & $98^{\circ} \mathrm{C}$ & $30 \mathrm{~s}$ & $30 \mathrm{x}$ \\
Annealing & $48-56^{\circ} \mathrm{C}$ & $35 \mathrm{~s}$ & \\
Elongation & $72^{\circ} \mathrm{C}$ & $30 \mathrm{~s}$ per $1 \mathrm{~kb}$ & \\
Final elongation & $72^{\circ} \mathrm{C}$ & $10 \mathrm{~min}$ & \\
Break & $4{ }^{\circ} \mathrm{C}$ & $\infty$ &
\end{tabular}

\section{Standard program for the DreamTaq polymerase}

\begin{tabular}{lll|l}
\hline Initial denaturation & $95^{\circ} \mathrm{C}$ & $5 \mathrm{~min}$ & \\
Denaturation & $95^{\circ} \mathrm{C}$ & $30 \mathrm{~s}$ & $30 \mathrm{x}$ \\
Annealing & $48-56^{\circ} \mathrm{C}$ & $35 \mathrm{~s}$ & \\
Elongation & $72^{\circ} \mathrm{C}$ & 1 min per $1 \mathrm{~kb}$ & \\
Final elongation & $72^{\circ} \mathrm{C}$ & 10 min \\
Break & $4{ }^{\circ} \mathrm{C}$ & $\infty$ & \\
\hline
\end{tabular}

\section{Long flanking homology PCR (LFH-PCR)}

The long flanking homology PCR (LFH PCR) was used to generate a DNA fragment for the deletion of genes in the genome of $B$. subtilis. Therefore, the upstream and downstream regions of the target genes are amplified by PCR (1000 bp). The genes encoding for the resistance against chloramphenicol, kanamycin, erythromycin, spectinomycin, tetracyclin and zeocin are amplified from the plasmids pGEM-cat, pDG780, pDG646, pDG1726, pDG1513 and pDG148 respectively. The flanking regions and the resistance cassette were fused together in the LFH PCR, in which the first step was the joining of the three fragments without the oligonucleotides. In a second step the oligonucleotides are added to the reaction and the complete fragment was amplified. Complementary sequences allow the joining of the fragments. Afterwards, B. subtilis competent cells were transformed with the LFH product and plated onto the respective selection plates. 
Reaction mix for LFH PCR with Phusion ${ }^{\mathrm{TM}}$ polymerase $(50 \mu \mathrm{l})$ :

$\begin{array}{ll}10 \mu \mathrm{l} & 5 x \text { HF reaction buffer } \\ 2 \mu \mathrm{l} & \text { dNTPs }(12.5 \mu \mathrm{mol} / \mathrm{ml}) \\ 4 \mu \mathrm{l} & \text { forward primer }(20 \mathrm{pmol}) \\ 4 \mu \mathrm{l} & \text { reverse primer }(20 \mathrm{pmol}) \\ 100 \mathrm{ng} & \text { upstream flanking region } \\ 100 \mathrm{ng} & \text { downstream flanking region } \\ 150 \mathrm{ng} & \text { resistance gene } \\ 1 \mu \mathrm{l} & \mathrm{Phusion}^{\text {TM }} \text { polymerase }(2 \mathrm{U} / \mu \mathrm{l}) \\ \text { Ad to } 50 \mu \mathrm{l} \text { with } \mathrm{dH}_{2} \mathrm{O}\end{array}$

Standard program for the LFH PCR with Phusion ${ }^{\mathrm{TM}}$ polymerase

\begin{tabular}{|c|c|c|c|c|}
\hline Initial denaturation & $98^{\circ} \mathrm{C}$ & \multicolumn{2}{|l|}{$3 \mathrm{~min}$} & \\
\hline Denaturation & $98^{\circ} \mathrm{C}$ & \multicolumn{2}{|l|}{$30 \mathrm{~s}$} & \\
\hline Annealing & $52^{\circ} \mathrm{C}$ & $35 \mathrm{~s}$ & & \\
\hline Elongation & $72^{\circ} \mathrm{C}$ & \multicolumn{2}{|l|}{$2 \min 15 \mathrm{~s}$} & \\
\hline Hold & $15^{\circ} \mathrm{C}$ & \multicolumn{2}{|l|}{$\infty$} & \\
\hline \multicolumn{5}{|c|}{ Addition of oligonucleotides } \\
\hline Denaturation & $98^{\circ} \mathrm{C}$ & \multicolumn{2}{|l|}{$30 \mathrm{~s}$} & \\
\hline Annealing & $52^{\circ} \mathrm{C}$ & \multicolumn{2}{|l|}{$35 \mathrm{~s}$} & $30 x$ \\
\hline Elongation & $72^{\circ} \mathrm{C}$ & \multicolumn{2}{|c|}{$3 \min 30 s+5 s /$ cycle } & \\
\hline Final elongation & $72^{\circ} \mathrm{C}$ & \multicolumn{3}{|l|}{$10 \min$} \\
\hline Break & $4^{\circ} \mathrm{C}$ & \multicolumn{3}{|l|}{$\infty$} \\
\hline
\end{tabular}

\section{Marker-free deletion system}

The marker-free deletion system is based on the mannose phosphotransferase system. In B. subtilis mannose uptake and phosphorylation is performed by the permease ManP. The resulting product mannose-6-phosphate is then further metabolized by the mannose-6-phosphate isomerase ManA. Without the corresponding genes $\operatorname{man} P$ and $\operatorname{man} A$, the uptake of mannose is not possible. However, if only manP is present, mannose-6-phosphate accumulates in the cell to a toxic level. The plasmids used in this method are pJOE6743 and pGP1022, which carry the manP gene as a counterselection marker and a spectinomycin resistance as a selection marker. 
The upstream and downstream regions of the deletion target were amplified, fused together and cloned into the deletion plasmid. A strain without the manP and manA genes ( $\triangle$ manP-manA) was transformed with the plasmid and plated on LB agar with spectinomycin. A $4 \mathrm{ml}$ LB culture was inoculated with a colony from the plates and after incubation at $37^{\circ} \mathrm{C}$ over the day, the cells were diluted $1: 10^{-4}$ In LB medium supplemented with $0.5 \%$ mannose. The cultures were incubated overnight, then diluted $2 \times 10^{-5}$ and plated onto LB agar plate with $0.5 \%$ mannose. The cells that could grow in the presence of mannose have lost the introduced manP gene and the uptake of mannose was not possible anymore. These colonies were again tested for their growth on LB medium with spectinomycin. Only the colonies which did not grow in the presence of spectinomycin were used for colony PCR to check for the correct deletion. This marker-free deletion method is illustrated in Figure 8 (Wenzel and Altenbuchner, 2015; Blötz et al., 2017).
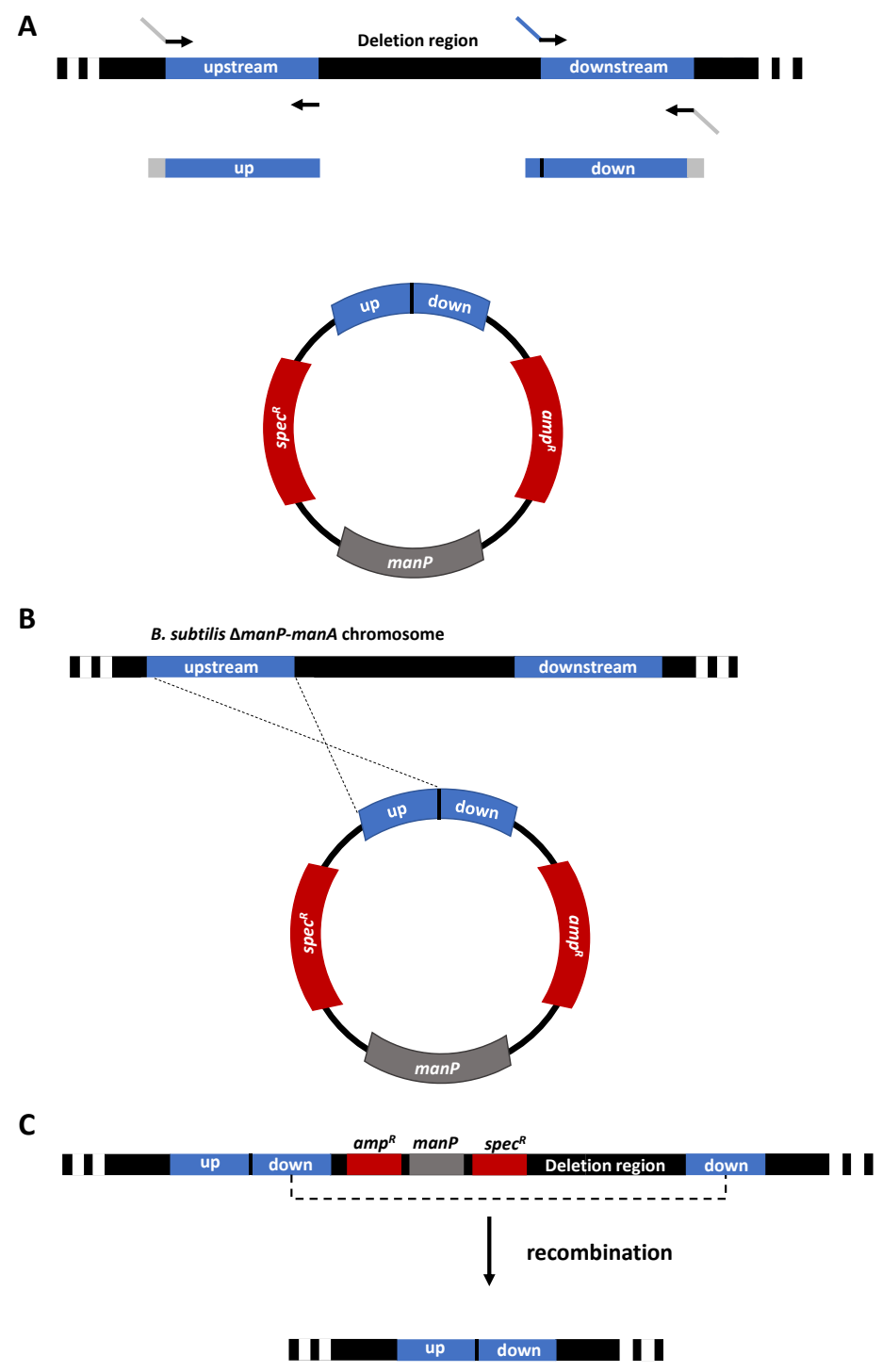

Figure 8: Marker-free deletion system. A The upstream and downstream regions of the deletion region were amplified, fused in an LFH reaction and cloned into the vector pJOE6743 or pGP1022. B The strain was transformed with the plasmid, which was inserted into the genome via one of the homolog flanks. C Since the flanking region, which was not used for the integration is now present in two copies, a recombination occurs, and the deletion region is removed. 


\section{Cre-lox deletion system}

The Cre-lox system is used to generate clean deletions. In the first step, the gene of interest is exchanged with a resistance cassette from an LFH product. The LFH product contains the up- and downstream regions from the gene of interest, a resistance cassette and additionally lox sites between the flanking regions and the resistance cassette. These 34 bp lox sites, lox71and lox66, can be recognized by the Cre recombinase from the P1 bacteriophage, which cuts and recombines these specific sequences. The gene encoding this enzyme was previously introduced into the sacA locus of the $B$. subtilis chromosome and the expression can be induced by the addition of xylose. If the expression of the cre recombinase is induced, the resistance cassette is cut out and the lox72 site remains in the genome (Figure 9). (Yan et al., 2008; Kumpfmüller et al., 2013).

A

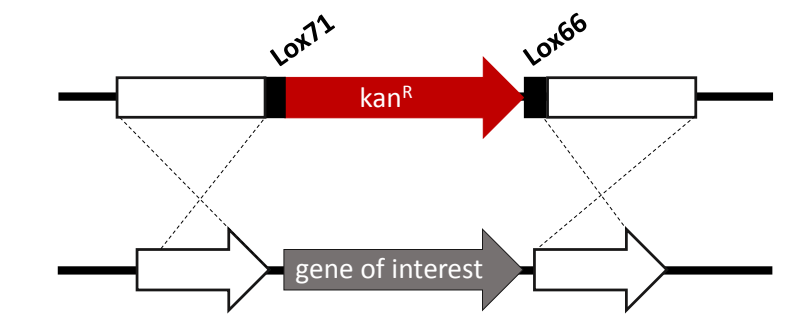

Chromosome with cre recombinase

B

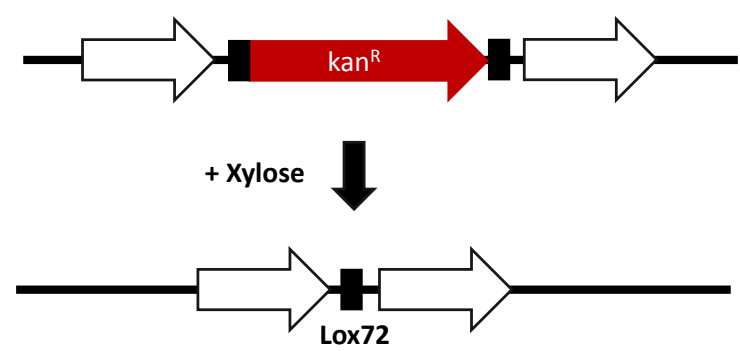

Figure 9: Cre-lox deletion. A The gene of interest was exchanged with an antibiotic resistance cassette via LFH. Additionally, lox sites were introduced. B The cre recombinase was induced by the addition of xylose and it cuts and recombines the lox71 and lox66 sites. Finally, the resistance cassette was removed and a lox72 site is left.

\subsubsection{Preparation and detection of proteins}

\section{Overexpression of recombinant proteins in E. coli}

The E. coli BL21 strain with the relevant plasmid was used to inoculate an overnight culture. The main culture of 1 I LB was inoculated to an $\mathrm{OD}_{600}$ of 0.1 and grown at $37^{\circ} \mathrm{C}$ and $200 \mathrm{rpm}$ until an optical density of 0.6 to 0.8 was reached. At this point, the inducer isopropyl- $\beta$-D-thiogalactopyranoside (IPTG) was added in a final concentration of $1 \mathrm{mM}$. 


\section{Cell disruption with French pressure cell press}

A cell pellet was resuspended in buffer $\mathrm{W}$ or ZAP buffer and filled into the precooled bomb. The remaining air was removed before the bomb was locked and placed in the French press. The disruption was performed three times with a pressure of $18.000 \mathrm{PSI}$.

\section{Preparation of crude extracts for $\beta$-galactosidase activity assay)}

The cell pellet, from a cell culture grown to an $\mathrm{OD}_{600}$ of $0.5-0.8$, was resuspended in $400 \mu \mathrm{l} Z$-buffer with $\beta$-mercaptoethanol and Lysozyme/DNase I mix (60 $\mu \mathrm{l}$ on $12 \mathrm{ml}$ Buffer). The samples were incubated $10 \mathrm{~min}$ at $37^{\circ} \mathrm{C}$ and $600 \mathrm{rpm}$ and afterwards centrifuged $3 \mathrm{~min}$ at $4^{\circ} \mathrm{C}$ and $14800 \mathrm{rpm}$.

\begin{tabular}{|c|c|c|}
\hline \multirow[t]{3}{*}{ LD mix } & $100 \mathrm{mg}$ & Lysozyme \\
\hline & $10 \mathrm{mg}$ & DNase I \\
\hline & \multicolumn{2}{|c|}{ Ad to $10 \mathrm{ml}$ with $\mathrm{dH}_{2} \mathrm{O}$} \\
\hline \multirow[t]{6}{*}{ Z-Buffer } & $0.534 \mathrm{~g}$ & $\mathrm{Na}_{2} \mathrm{HPO}_{4} \times 2 \mathrm{H}_{2} \mathrm{O}$ \\
\hline & $0.276 \mathrm{~g}$ & $\mathrm{NaH}_{2} \mathrm{PO}_{4}$ \\
\hline & $0.037 \mathrm{~g}$ & $\mathrm{KCl}$ \\
\hline & $50 \mu \mathrm{l}$ & $1 \mathrm{M} \mathrm{MgSO}_{4}$ \\
\hline & $175 \mu l$ & $\beta$-mercaptoethanol \\
\hline & \multicolumn{2}{|c|}{ Ad to $50 \mathrm{ml}$ with $\mathrm{dH}_{2} \mathrm{O}$} \\
\hline
\end{tabular}

\section{Purification of Strep-tagged proteins}

A cell pellet of the respective $E$. coli strain was resuspended in cold buffer $W$ and the cells were disrupted with the french pressure cell press as described above. The solution was centrifuged at $35000 \mathrm{rpm}$ and $4^{\circ} \mathrm{C}$ for $30 \mathrm{~min}$ to remove cell debris. This crude extract was then loaded onto preequilibrated $500 \mu$ l Strep-Tactin Sepharose (IBA) in a Poly-Prep chromatography column (Biorad). Buffer $\mathrm{W}$ was used to wash the mixture five times and the bound proteins were then eluted with buffer $E$ in four fractions. The fractions were analysed by SDS page (Blötz et al., 2017).

\begin{tabular}{|c|c|c|}
\hline \multirow[t]{5}{*}{ Buffer W (pH 8.0) } & $121.14 \mathrm{~g}$ & Tris-base \\
\hline & $87.7 \mathrm{~g}$ & $\mathrm{NaCl}$ \\
\hline & $3.72 \mathrm{~g}$ & $\mathrm{Na}_{2} \mathrm{EDTA}$ \\
\hline & \multicolumn{2}{|c|}{ Adjust the $\mathrm{pH}$ with $\mathrm{HCl}$ to 8.0} \\
\hline & \multicolumn{2}{|c|}{ Ad to $1000 \mathrm{ml}$ with $\mathrm{dH}_{2} \mathrm{O}$} \\
\hline
\end{tabular}




\section{$\begin{array}{lll}\text { Buffer E } & 0.027 \mathrm{~g} & \text { D-Desthiobiotin }\end{array}$}

Add $50 \mathrm{ml} 1 \mathrm{x}$ buffer $\mathrm{W}$

\section{Purification of His-tagged proteins}

An E. coli cell pellet was resuspended in ZAP Buffer and the cells were disrupted as described above. To remove cell debris, the cell solution was centrifuged at $17.500 \mathrm{rpm}$ and $4^{\circ} \mathrm{C}$ for $30 \mathrm{~min} .1 .25 \mathrm{ml}$ of $\mathrm{Ni}_{-} \mathrm{NTA}^{\circledR}$ sepharose was loaded onto a column and equilibrated with $12.5 \mathrm{ml}$ ZAP Buffer. The crude extract was loaded onto the column and the flow through was collected. Five washing steps were performed with each $10 \mathrm{ml}$ ZAP buffer and the elution was done with ZAP buffer with increasing concentrations of imidazole (Blötz et al., 2017).

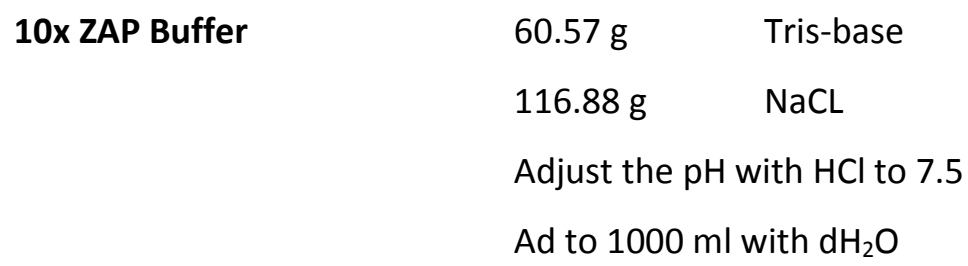

\section{Dialysis}

The dialysis was used to remove the desthiobiotin from the protein solution or to change to a desired buffer. Therefore, the elution fractions with the highest protein amount were pipetted into a dialysis tube and dialyzed against the desired buffer in an excess of 1000 fold overnight.

\section{Denaturing gel electrophoresis of proteins (SDS-PAGE)}

The sodium dodecyl sulfate polyacrylamide gel electrophoresis (SDS-PAGE) method described by Laemmli (1970) was used to analyse the protein sizes. First the protein samples were mixed with $5 x$ SDS loading dye and denatured for $10 \mathrm{~min}$ at $95^{\circ} \mathrm{C}$. The SDS gels consist of a stacking gel with $5 \%$ polyacrylamide and a running gel with $12 \%$ polyacrylamide underneath. The samples and a protein size marker PageRuler ${ }^{\mathrm{TM}}$ Plus prestained were loaded onto the gel and the electrophoresis was performed at 100-160 (Blötz et al., 2017).

$\begin{array}{lll}\text { 5x SDS loading dye } & 1.4 \mathrm{ml} & \text { Tris- } \mathrm{HCl}(1 \mathrm{M}, \mathrm{pH} 7.0) \\ 3 \mathrm{ml} & \text { Glycerol (100\%) } \\ 2 \mathrm{ml} & \text { SDS (20\%) } \\ 1.6 \mathrm{ml} & \beta \text {-Mercaptoethanol (100\%) } \\ 0.01 \mathrm{~g} & \text { Bromphenol blue } \\ 2 \mathrm{ml} & \mathrm{dH}_{2} \mathrm{O}\end{array}$


10x Page buffer (PLP)

$\begin{array}{ll}144 \mathrm{~g} & \text { L-glycine } \\ 30 \mathrm{~g} & \text { Tris-base } \\ 10 \mathrm{~g} & \text { SDS }\end{array}$

$12 \%$ running gel

$\begin{array}{ll}4.8 \mathrm{ml} & \mathrm{dH}_{2} \mathrm{O} \\ 3.9 \mathrm{ml} & \text { Tris-HCl (1.5 M, pH 8.8) } \\ 6 \mathrm{ml} & \text { Acryl-bisacrylamide (30\%) } \\ 150 \mu \mathrm{l} & \text { SDS (10\%) } \\ 150 \mu \mathrm{l} & \text { Ammonium persulfate (10\%) } \\ 15 \mu \mathrm{l} & \text { TEMED }\end{array}$

5\% stacking gel

$\begin{array}{ll}10.25 \mathrm{ml} & \mathrm{dH}_{2} \mathrm{O} \\ 1.305 \mathrm{ml} & \text { Tris-HCl (1.5 M, pH 6.8) } \\ 1.95 \mathrm{ml} & \text { Acryl-bisacrylamide (30\%) } \\ 150 \mu \mathrm{l} & \text { SDS (10\%) } \\ 150 \mu \mathrm{l} & \text { Ammonium persulfate (10\%) } \\ 30 \mu \mathrm{l} & \text { TEMED }\end{array}$

\section{Coomassie staining}

After the SDS-Page, the protein gels were stained with Coomassie Brilliant Blue. Therefore, the gels were first treated with a fixation solution for $30 \mathrm{~min}$ at RT. A staining solution was used to stain the proteins. Afterwards, the gels were destained with water (Blötz et al., 2017).

$\begin{array}{lll}\text { Fixation solution } & \begin{array}{l}10 \% \\ 50 \%\end{array} & \begin{array}{l}\text { Acetic acid } \\ \text { Methanol }\end{array} \\ & \text { Ad to the final volume with } \mathrm{dH}_{2} \mathrm{O}\end{array}$




\section{Enzyme activity assays}

\section{$\beta$-galactosidase activity assay}

For the measurement of the $\beta$-galactosidase activity, $100 \mu \mathrm{l}$ of the crude extract was mixed with $700 \mu \mathrm{l}$ Z-Buffer with $\beta$-mercaptoethanol and incubated for $5 \mathrm{~min}$ at $28^{\circ} \mathrm{C}$. The reaction was initiated with the addition of $200 \mu$ l o-nitrophenyl- $\beta$-D-galactopyranoside (ONPG). The reaction is stopped with $500 \mathrm{ml} 1 \mathrm{M} \mathrm{Na}_{2} \mathrm{CO}_{3}$ when the solution turns yellow. To detect the amount of produced onitrophenyl, the $\mathrm{OD}_{420}$ of the samples was measured. $10 \mu \mathrm{l}$ of the crude extract was used for the Bradford assay to determine the protein concentration. The $\beta$-galactosidase activity was calculated with the following formula:

$$
\frac{O D 420 \mathrm{~nm}}{\Delta t \times O D 595 \mathrm{~nm}} \times 2005.3475
$$

\begin{tabular}{|c|c|c|}
\hline \multirow[t]{2}{*}{ ONPG } & $4 \mathrm{mg}$ & o-Nitrophenyl- $\beta$-D-Galactopyranoside \\
\hline & $1 \mathrm{ml}$ & Z-Buffer without $\beta$-mercaptoethanol \\
\hline \multirow[t]{2}{*}{$1 \mathrm{M} \mathrm{Na}_{2} \mathrm{CO}_{3}$} & $26.5 \mathrm{~g}$ & $\mathrm{Na}_{2} \mathrm{CO}_{3}$ \\
\hline & Ad to 2 & th $\mathrm{dH}_{2} \mathrm{O}$ \\
\hline
\end{tabular}

\section{Citrate synthase activity assay}

For the determination of citrate synthase activity, a colorimetric assay was performed. The citrate synthase converts acetyl-CoA and oxaloacetate to citrate and coenzyme A. This coenzyme A reacts in the assay with the Ellman's reagent 5,5'-dithiobis (2-nitrobenzoic acid) (DTNB) and the resulting compound TNB can be measured spectrophotometrically at $412 \mathrm{~nm}$. The reaction mixture contains $100 \mathrm{mM}$ Tris- $\mathrm{HCl}$ (pH 8.0), $1 \mathrm{mM}$ DTNB and $50 \mathrm{nM}$ of the purified citrate synthases CitZ or CitA. The reaction was initiated by the addition of $0.3 \mathrm{mM}$ oxaloacetate and $0.3 \mathrm{mM}$ acetyl-CoA and the absorbance at $412 \mathrm{~nm}$ was measured at $25^{\circ} \mathrm{C}$. For the determination of $\mathrm{Km}$ and Vmax values, one substrate was added in a constant concentration and the other substrate was added in varying concentrations (0.03-0.45 mM) (Ellman, 1959; Johansson and Pettersson, 1974).

From the resulting data, the initial reaction rate $v_{0}$ was determined as the change of absorption $(\Delta A)$ per minute. The next step was to plot the values $1 / v_{0}$ against $1 /$ substrate concentration [S] in a Linewaever-Burk diagram (Lineweaver and Burk, 1934). From this plot, the $\mathrm{Km}$ and Vmax values can be determined by the following equation:

$$
\frac{1}{v_{0}}=\frac{K_{M}}{V_{\max }[S]}+\frac{1}{V_{\max }}
$$




\section{Hom assay}

For the measurement of Hom enzyme activity, the reverse reaction from L-homoserine to Laspartate 4-semialdehyde was used. The simultaneous conversion of $\mathrm{NADP}^{+}$to NADPH and the accompanying change in absorption at $340 \mathrm{~nm}$ was measured with a photometer. The reaction mixture contains $100 \mathrm{mM}$ Tris- $\mathrm{HCl}(\mathrm{pH} 7.5), 50 \mathrm{mM} \mathrm{KCl}, 1 \mathrm{mM} \mathrm{NADP}{ }^{+}$and $0.02 \mathrm{mM}$ DTT. To initiate the reaction, $10 \mathrm{mM}$ homoserine was added to the reaction. The change in absorption at $340 \mathrm{~nm}$ was measured at 25 or $37^{\circ} \mathrm{C}$ (Hama et al., 1990; Hama et al., 1991).

\section{Preparation of samples for proteome analysis}

A $4 \mathrm{ml}$ overnight culture was used to inoculate a $50 \mathrm{ml} \mathrm{LB}$ preculture to an $\mathrm{OD}_{600}$ of 0.05 and incubated at $37^{\circ} \mathrm{C}$ and $200 \mathrm{rpm}$ until an $\mathrm{OD}_{600}$ of 0.5 is reached. From this preculture, a main culture of $150 \mathrm{ml}$ in a 1 I flask was inoculated to an $\mathrm{OD}_{600}$ of 0.05 . After incubation to an $\mathrm{OD}_{600}$ of $0.5,30 \mathrm{ml}$ of the cells were harvested by centrifugation at $4^{\circ} \mathrm{C}$ for $15 \mathrm{~min}$ and $8500 \mathrm{rpm}$. The supernatant was removed and the cells were washed in $10 \mathrm{ml}$ TE Buffer. The solution was again centrifuged as described and the supernatant was removed. The samples were frozen in liquid nitrogen and analysed by the Department of Functional Genomics in Greifswald. The analysis was performed as described in Reuß et al. (2017).

\subsubsection{Drop dilution assay}

Overnight cultures of the strains are used to inoculate a $4 \mathrm{ml}$ cultures in either LB or C-glc medium, that were inoculated at $37^{\circ} \mathrm{C}$ and $200 \mathrm{pm}$ until an $\mathrm{OD}_{600}$ of around 1 is reached. The cells were washed and resuspended in $\mathrm{C}$-glc medium to a final $\mathrm{OD}_{600}$ of 1 . These samples were used to prepare serial dilutions of $10^{-1}$ to $10^{-6}$ and $5 \mu$ l of each dilution was dropped on the respective plates. The plates were incubated at $37^{\circ} \mathrm{C}$ for two days in case of the $\mathrm{C}$-glc minimal plates with serine.

\subsubsection{Sporulation assay}

From a $4 \mathrm{ml} \mathrm{LB}$ overnight culture of the $B$. subtilis strain, a $4 \mathrm{ml} \mathrm{SP}$ culture was inoculated 1:100 and incubated at $37^{\circ} \mathrm{C}$ and $200 \mathrm{rpm}$ until it reached an $\mathrm{OD}_{600}$ of $0.2-0.4$. This culture was further used to inoculate a new $4 \mathrm{ml} \mathrm{SP}$ culture 1:100, which was inoculated for at least $20 \mathrm{~h}$. The $\mathrm{OD}_{600}$ of the culture was measured and a cell solution of OD 1 was prepared in $0.9 \% \mathrm{NaCl}$ solution. Two samples of each $1 \mathrm{ml}$ were prepared. To remove all living cells from the culture, a heating step at $85^{\circ} \mathrm{C}$ for 30 min was applied to one sample. The heated and the not heated samples were diluted step-bystep $10^{-1}$ in a drop dilution assay in $0.9 \% \mathrm{NaCl}$ and plated on LB plates. The plates were incubated at $37^{\circ} \mathrm{C}$ over night. 


\section{Results}

\subsection{The MiniBacillus project}

The aim of the MiniBacillus project is to understand what is needed for life, by reducing the wellknown genome of $B$. subtilis. A minimal set of genes should remain in the genome, that were defined in a blueprint (Reuß et al., 2016). For the genome reduction, chromosomal regions with unnecessary genes are deleted step by step with a marker-free deletion system (Wenzel and Altenbuchner, 2015). The MiniBacillus strain PG10, with a genome reduction of $34.54 \%$ was analysed with a multi-omics approach (Reuß et al., 2017). Therefore, this strain is very well characterized. This work is based on an offspring of this strain, the strain PG18. PG18 has a genome reduction of $36.61 \%$ (Reuß, 2017).

\subsubsection{The deletion progress}

Previous transcriptome data showed, that some genes are highly expressed in the MiniBacillus strain (Table 2). Part of these is the mhqNOP operon, which was over 4700 -fold upregulated. This was caused by the deletion of the repressor MhqR, which normally regulates the resistance to quinones and diamide (Töwe et al., 2007; Reuß et al., 2017). However, the upregulated expression wastes a lot of energy and leads to an imbalance in the cell. Furthermore, the function of the mhqNOP genes is the protection against methyl-hydroquinone, which is unnecessary for the final MiniBacillus strain. Therefore, the plasmid pGP2093 was used to delete the mhqNOP operon in the strain PG18 with the marker-free deletion system as described in chapter 2.2.6., resulting in the strain PG29.

Table 2: Operons that are higher expressed in PG10, compared to $\Delta 6$

\begin{tabular}{|l|l|l|l|}
\hline Operon & Function & Regulators & Factor \\
\hline mhqNOP & Protection against hydroquinone & MhqR (deleted) & 4781 \\
\hline paiAB & $\begin{array}{l}\text { Control of intracellular polyamine } \\
\text { concentrations }\end{array}$ & & 420 \\
\hline
\end{tabular}

The next step was to delete the paiAB operon with the plasmid pGP2094. This operon encodes for a spermine/ spermidine-N-acetyltransferase, which is also upregulated 420 -fold. The resulting strain PG30 was further used to restore a point mutation in the pit gene, a low-affinity phosphate transporter. This point mutation was noticed in PG10 and leads probably to a reduction of phosphate uptake. However, this could be detrimental for the strain, since the reduced level of phosphate in the cell might activate a regulator system for phosphate metabolism, the PhoPR 
system. This leads to the activation of genes for the acquisition of phosphate and similar to the repression of the tagAB and tagDEFGH operons for the biosynthesis of teichoic acids (Prágai et al., 2004). Therefore, the pit point mutation was restored in strain PG31.

Since the essential or for the MiniBacillus necessary genes are scattered around the genome and the deletion regions become smaller, an approach to accelerate the deletion process might be the defragmentation. Functionally related genes, which should remain in the MiniBacillus, are clustered together in one locus. The native locus is then dispensable and can be deleted in one bigger deletion, instead of two smaller ones. This clustering can be done by introducing a second copy of the gene. In this case a glycolytic cassette should be introduced, containing the genes pgi, fbaA, ptsGHI. Pgi is the glucose-6-phosphate isomerase and FbaA the fructose-1,6-bisphophate aldolase and both enzymes are part of glycolysis, which plays a central role in the MiniBacillus blueprint. The operon ptsGHI encodes for the glucose phosphotransferase system which is responsible for the uptake of glucose in the cell. The introduction of this glycolytic cassette was shown to be functional in Zschiedrich (2014), however, the transfer of this construct would lead to the deletion of several genes including the gene $n r n A$. The deletion of $n r n A$, encoding for a nanoRNase, leads to the reduction of the competence in the MiniBacillus strain (Reuß, 2017). Therefore, the glycolytic cassette was newly assembled and introduced next to $n r n A$, to sustain competence. This was done in two steps as shown in Figure 10. First the genes pgi and fbaA, together with a chloramphenicol resistance were introduced with a PCR product next to dnaE, leading to the deletion of the unknown gene $y \operatorname{trH}(\mathrm{PG} 32)$. In the second step, the operon ptsGHI with a kanamycin resistance cassette was exchanged with the chloramphenicol resistance and the genes $y t r l$ and $y t z J$. The resulting strain PG33 was selected on plates with kanamycin and also tested for the loss of the chloramphenicol resistance. The genes pgi, fbaA and ptsGHI can now be deleted at their native locus to fasten the deletion process.

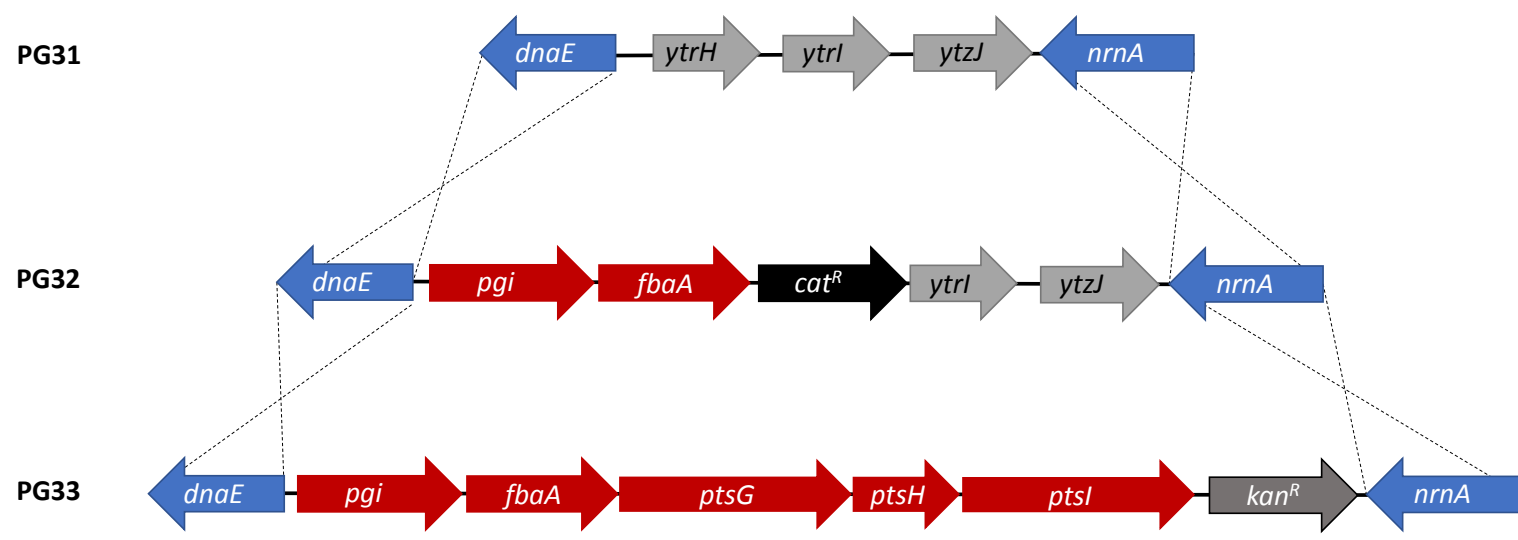

Figure 10: The two steps of the glycolytic cassette introduction into the MiniBacillus strain. First, the genes pgi and fbaA were integrated with a chloramphenicol cassette and in second step the genes ptsGHI were introduced with a kanamycin resistance. 
The following deletions from PG34 to 39 are deletions of genomic regions that are not necessary for the final MiniBacillus strain. The deletions are listed in Table 3, together with the plasmids that were used and the resulting genome size. The consequences of these deletions and the deletions that were already done before are discussed in chapter 3.1.3. The strains PG34, PG37 and PG39 were additionally analysed by WGS.

Table 3: The MiniBacillus deletion strains constructed in this work

\begin{tabular}{|c|c|c|c|c|c|}
\hline strain & $\begin{array}{l}\text { Genome } \\
\text { size }\end{array}$ & $\begin{array}{l}\text { Deletion } \\
\text { plasmid }\end{array}$ & Deletion & $\begin{array}{l}\text { \% reduction in } \\
\text { comparison to } \\
\text { wild type }\end{array}$ & $\begin{array}{l}\text { WGS } \\
\text { analysis }\end{array}$ \\
\hline PG10 & 2759359 & & Reuß et al. (2017) & 34.54 & yes \\
\hline PG18 & 2672270 & & Reuß (2017) & 36.61 & yes \\
\hline PG29 & 2670199 & pGP2093 & $\Delta m h q N O P$ & 36.66 & no \\
\hline PG30 & 2666896 & pGP2094 & yuzG-sufA & 36.74 & no \\
\hline PG31 & 2666876 & pJOE3256 & pit point mutation & 36.74 & yes \\
\hline PG32 & 2670874 & PCR product & $\begin{array}{l}\text { insertion pgi, fbaA, } \\
\text { cat; Deletion ytrH }\end{array}$ & 36.64 & no \\
\hline PG33 & 2674507 & PCR product & $\begin{array}{l}\text { insertion ptsGHI, } \\
\text { kan; Deletion ytrl, } \\
y t z J, c a t\end{array}$ & 36.56 & no \\
\hline PG34 & 2652827 & pGP2098 & ycgQ-yckE & 37.07 & yes \\
\hline PG35 & 2635284 & pGP2088 & yvaM-yvbK & 37.49 & no \\
\hline PG36 & 2622356 & pGP2073 & nhaX-yhaX & 37.79 & no \\
\hline PG37 & 2587747 & pGP2270 & $g l p Q-y c b K$ & 38.62 & yes \\
\hline PG38 & 2554562 & pGP2282 & yqjF-yqjG & 39.40 & no \\
\hline PG39 & 2507732 & pGP2283 & $y d d N-y d f M$ & 40.51 & yes \\
\hline
\end{tabular}

The final MiniBacillus strain of this work PG39 has a genome size of about $2.5 \mathrm{Mbp}$, which corresponds to a genome reduction of $40.51 \%$ in comparison to the wild type strain 168 . This is the greatest reduction of the $B$. subtilis genome published so far. 


\subsubsection{Phenotypical analysis}

The final MiniBacillus strain should sustain a robust growth at $37^{\circ} \mathrm{C}$ in LB-glc medium. To verify that the constructed strains are still able to grow under these conditions, the growth was monitored in $15 \mathrm{ml} \mathrm{LB}$-glc medium at $37^{\circ} \mathrm{C}$ (Figure $11 \mathrm{~A}$ ).

A

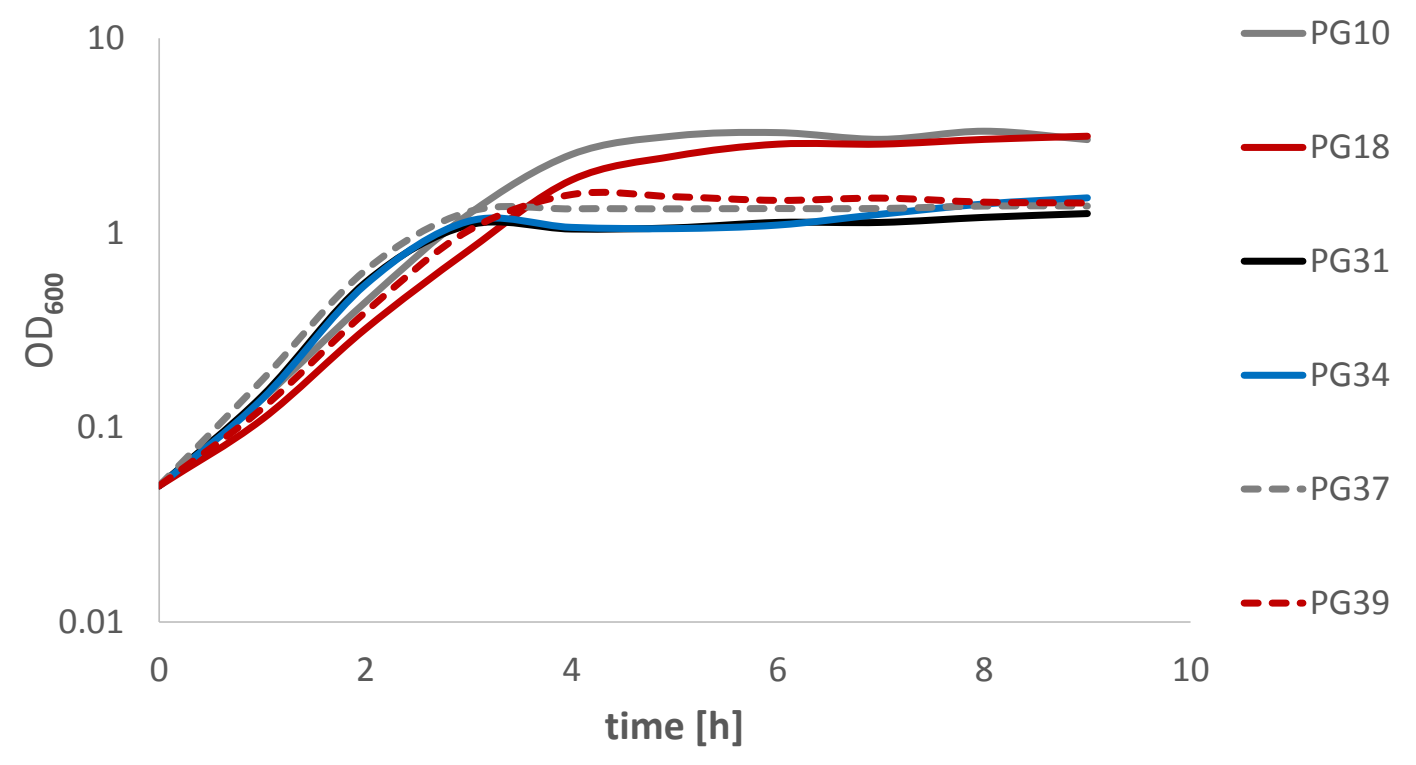

B $1 \mathrm{~h}$ $3 \mathrm{~h}$ $5 \mathrm{~h}$ $7 \mathrm{~h}$ $9 \mathrm{~h}$

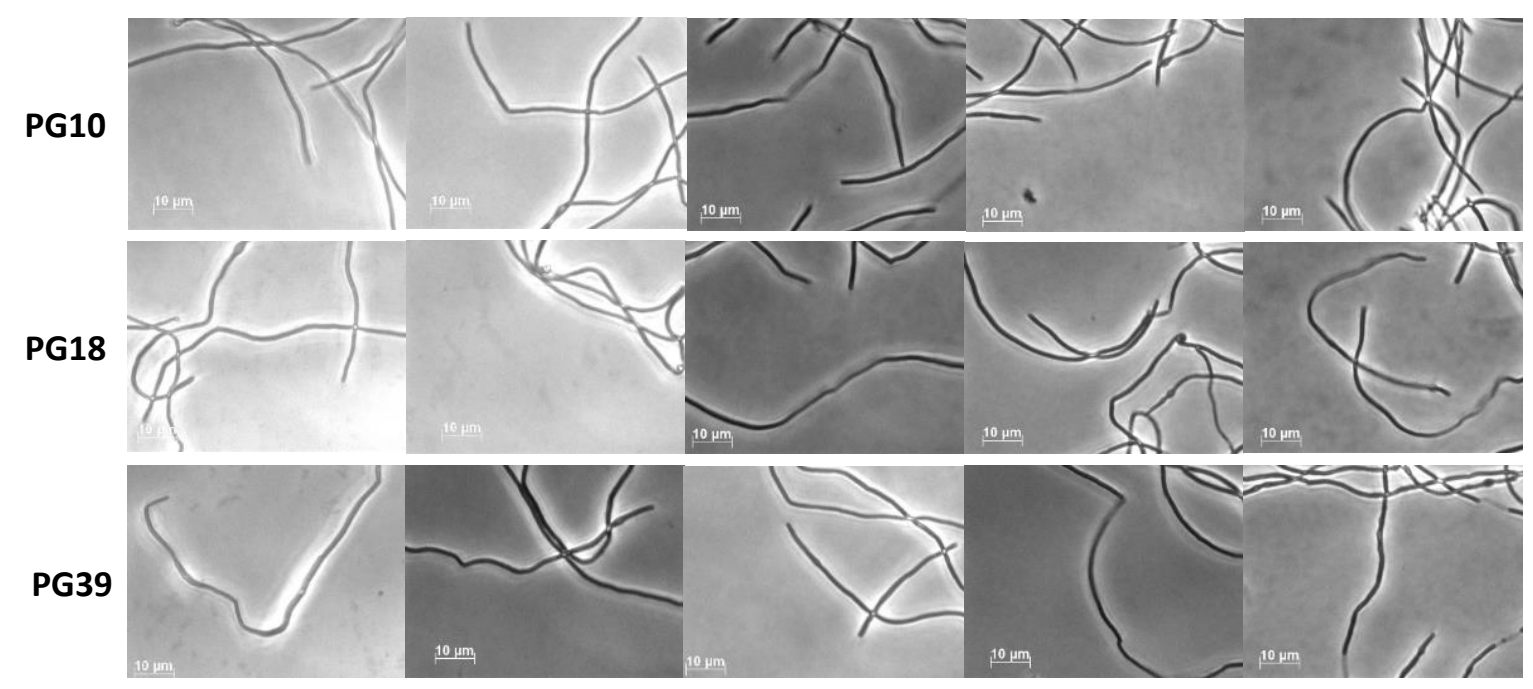

Figure 11: The growth of the MiniBacillus deletion strains in LB-glc. A The strains were grown in $15 \mathrm{ml} \mathrm{LB-glc}$ medium in baffled flask at $37^{\circ} \mathrm{C}$. B Samples from the LB-glc cultures were analysed under the microscope every two hours.

The tested strains PG10, PG18, PG31, PG34, PG37 and PG39 show the same growth rate in LB-glc medium. However, the ancestor strains PG10 and PG18 are able to reach a higher OD in comparison to the other strains. These strains seem to produce a higher biomass than the others. Since the growth rate is more important for the project and the difference in the final OD is not severe. 
Furthermore, the cell morphology was also observed at several time points from the growth curve (Figure $11 \mathrm{~B}$ ). The microscopy pictures show the same cell morphology for all strains. Normally, cells of the B. subtilis wild type strain 168 are rod-shaped single cells. Additionally, they are able to form spores. For the MiniBacillus strains, long cell chains without spores can be observed. If the cells are treated with a membrane dye, it can be observed that these are indeed long chains of single cells and not several elongated cells (Figure 12). However, this formation of long cell chains is a phenotype that was already observed very early in the deletion process and the reason for this is not known (Reuß, 2017). But since the cell morphology of PG39 shows no differences to that of PG18 or PG10, the additional deletions seem to have no influence on it.
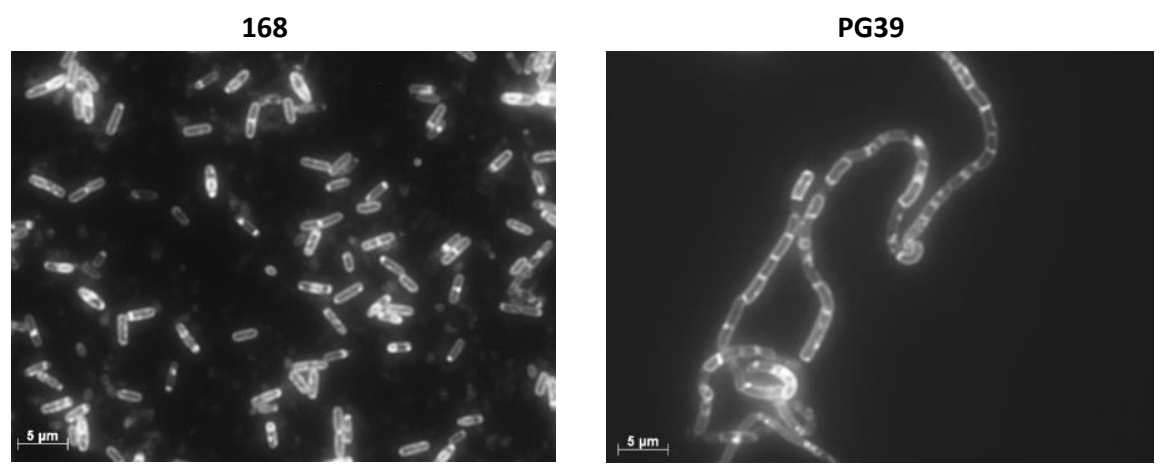

Figure 12: The comparison of the cell morphology of the wild type strain and the strain PG39. The cells were treated with the membrane dye FM 4-64 to visualize the cell membrane.

In a previously constructed MiniBacillus strain, the problem of genomic instability occurred and the reason for that was unknown (Reuß, 2017). However, to sustain a stable DNA replication process, the basic structure of the chromosome should be maintained. The origin and the termination of replication are located on opposite sides of the genome. In Figure 13 it can be seen, that the deletion regions are scattered around the genome and that the balance between the origin and the termination of replication is sustained.

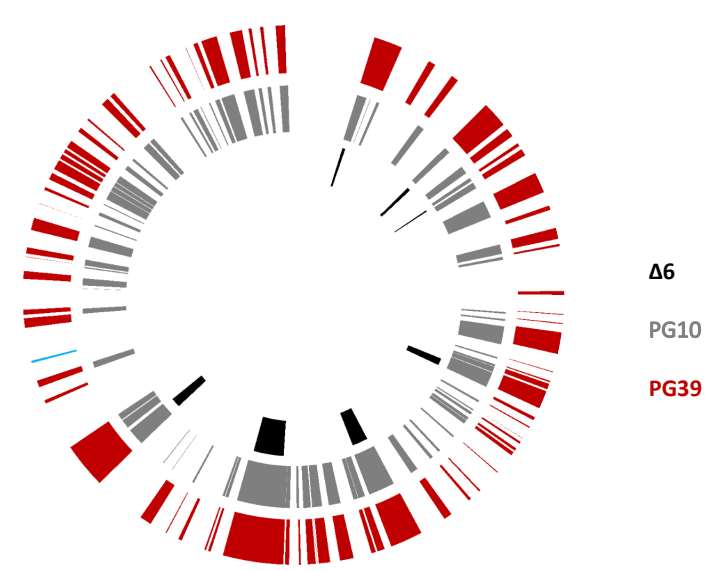

Figure 13: The chromosomes of the strains $\Delta 6$, PG10 and PG39. The deleted regions are shown, which are scattered around the genome. 
Previously, multi-omics experiments were performed for the strain PG10 in comparison to the ancestor strain $\Delta 6$. A lot of information can be obtained from these experiments. Therefore, proteome analysis was performed for the strains $\Delta 6, \mathrm{PG} 10$ and PG38. Furthermore, the genes that remained in the genome were divided into the five categories: cellular processes, metabolism, information processing, lifestyles and others. The results of the gene categorization and the proteome analyse are shown in Figure 14.

\section{A Gene count}

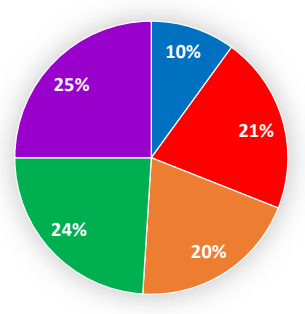

B Relative protein fraction

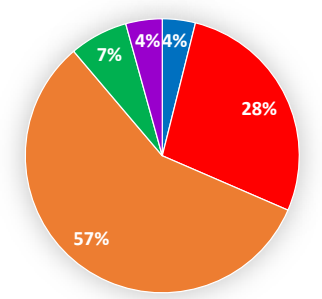

$\Delta 6$
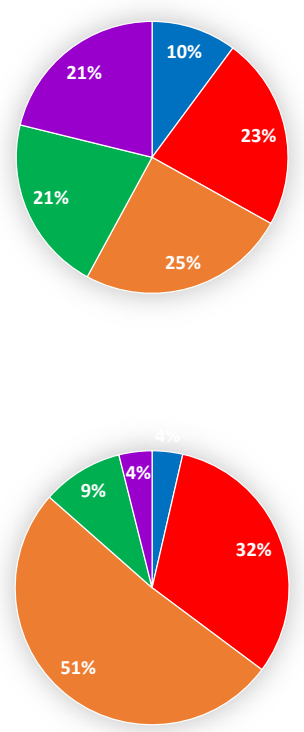

PG10

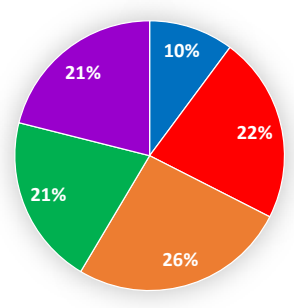

- Cellular processes

- Metabolism

Information processing

- Lifestyles

- Other

Figure 14: The relative results of the proteome analysis and the categorization of the genes. A The remaining genes in the genome are divided into the five different categories. $\mathbf{B}$ The relative protein fractions that were determined are shown for the five functional categories. (Data processing: Bingyao Zhu)

Around $2 \%$ of the genes in the chromosome of strain $\Delta 6$ are encoding for lifestyle genes. However, this number is reduced in PG10 and PG38 to 21\%, since most of the genes responsible for the sporulation and other not necessary processes are deleted. The final MiniBacillus strain will not need several pathways for different lifestyles. In contrast, many of the genes from the information process are needed to sustain life. This is reason why the percentage of these genes even increase in the relative gene count. The relative protein fractions for each category was determined from the proteome data. Interestingly, the amount of proteins in the cell involved in the information processing is the biggest fraction with $57 \%$ in the strain $\Delta 6$. Although the genes in this fraction make up only $20 \%$. This was already observed by Reuß et al., (2017). Therefore, the relative protein fractions for cellular processes, lifestyle and other are in comparison smaller. However, the percentages for each fraction are not changing significantly in PG10 and PG39. This means that there are no major changes in the proteome balance. However, some proteins show a different 
protein count in PG39 in comparison to PG10, there seems to be no significant up- or downregulation of a specific pathway (data not shown).

\subsubsection{Deletion impact}

Although the PG39 MiniBacillus strain shows almost no phenotypic difference in comparison to the PG18 ancestor strain in LB-glc medium, the deletions could have an impact under other conditions. First of all, the complex metabolism of $B$. subtilis was reduced. Since the known amino acid importers should remain in the cell, the biosynthesis pathways become dispensable. Therefore, some biosynthesis genes are already deleted, which means that the strain is already auxotrophic for the amino acids tryptophan, valine, isoleucine, leucine, proline and arginine. Furthermore, in PG38, the biosynthesis genes for biotin (bioWAFDBI) were deleted, which means the biotin importer YhfU is now essential for the cell. The strain is biotin auxotroph. B. subtilis wild type cells are able to use different carbon sources. In contrast, the MiniBacillus strain has some deletions in the uptake systems for several carbon sources (Table 4).

Table 4: The deletion impact on strain PG39

\begin{tabular}{|l|l|l|}
\hline Auxotrophic for & $\begin{array}{l}\text { No longer utilizable carbon } \\
\text { sources }\end{array}$ & $\begin{array}{l}\text { Deleted genes coding for } \\
\text { sigma factors }\end{array}$ \\
Valine & Maltose & sigE - sporulation \\
Isoleucine & Oligo-ß-glucoside & sigG - sporulation \\
Leucine & Oligo-ß-mannoside & sigF - sporulation \\
Proline & Fructose & sigZ - ECF-sigma factor \\
Arginine & Inositol & \\
& Arabinose & \\
Biotin & Ribose & \\
& Xylose & \\
\hline
\end{tabular}

For the final MiniBacillus strain, the alternative sigma factors of no interest. Therefore, PG39 has already deleted some of the responsible genes that are listed in Table 4. However, this has also an effect on the lifestyle category, since less stress responses are available. Furthermore, a lot of genes related to sporulation were deleted so that PG39 forms no spores anymore. However, the main part of the deleted genes are of unknown function.

The WGS data of the strains PG10, PG18, PG31, PG34, PG37 and PG39, were analysed for potential mutations that occured during the deletion process. All single nucleotide polymorphisms 
(SNPs) and variations were compared to the strain PG10, which was already analysed (Reuß et al., 2017). Potential mutations that are often found during WGS of $B$. subtilis strains are rejected. Some mutations were found for the strains PG18, PG31 and PG39 which are listed in Table 5.

Table 5: The mutations found in the MiniBacillus WGS data in comparison to PG10

\begin{tabular}{|l|l|l|}
\hline Strain & Mutation & function \\
\hline PG18 & Promoter tagAB & Biosynthesis of teichoic acid \\
\cline { 2 - 3 } & Promoter ywaG & Putative transcriptional regulator \\
\cline { 2 - 3 } & YqgS L220LTEM (Insertion) & Minor lipoteichoic acid synthase \\
\hline PG31 & MtIA M11R & Mannitol-specific permease \\
\cline { 2 - 3 } & YgaE F31S & Unknown \\
\cline { 2 - 3 } & YhbD G152S & Unknown \\
\hline PG39 & AmtB A76V & Ammonium transporter \\
\cline { 2 - 3 } & mfd (bp 475 deleted $\rightarrow$ frameshift) & Transcription-repair coupling factor \\
\cline { 2 - 3 } & YqgN T9A & Unknown \\
\hline
\end{tabular}

Some mutations were found in genes with unknown function. In the strain PG18, the promoter of the $\operatorname{tag} A B$ operon is mutated. These genes are essential and encode for proteins involved in the biosynthesis of teichoic acid, which is an important component of the cell wall. The mutation is a substitution of $\mathrm{G}$ to $A 69 \mathrm{bp}$ in front of the tagA gene, but it is not located in the -35 or -10 region of the promoter. It was previously described, that the $\operatorname{tag} A B$ operon is repressed by PhoPR system (Liu et al., 1998). This system is activated by phosphate starvation (Prágai et al., 2004). It was already described that the MiniBacillus strains from PG10 to PG31 harbour a point mutation in the lowaffinity transporter Pit. This mutation might have reduced the phosphate level in the cell, by which the PhoPR system was activated. Therefore, the $\operatorname{tag} A B$ operon expression was repressed. But the cell needs to synthesize the essential cell wall component lipoteichoic acid. Although, the mutation in the promoter region of $\operatorname{tag} A B$ is not in the known PhoP binding sides, it could have led to a derepression of the operon. Interestingly, the mutated gene yqgS is also involved in the synthesis of lipoteichoic acid. But the resulting enzyme is only a minor lipoteichoic acid synthase and it is only active during sporulation (Wörmann et al., 2011).

The mutation in $m t / A$, encoding for the mannitol-specific permease, is interesting for the MiniBacillus project, since the comKS system is induced by mannitol. Although, the effect of several mutations on the mannitol-specific phosphotransferase system (PTS) was already studied, it is not known which effect this mutation might cause (Bouraoui et al., 2013). Another transporter was also 
found to be mutated in PG39, the ammonium transporter AmtB. But the gene amtB is not part of the final MiniBacillus blueprint.

To conclude, the current MiniBacillus strain PG39 has a genome reduction of $40.51 \%$ and shows almost no differences in growth and cell morphology. However, the strain is auxotrophic for several amino acids and the import systems for several carbon sources are deleted. The deletions also led to the accumulation of some mutations.

\subsection{The role of the TCA cycle in the MiniBacillus project}

The TCA cycle is a central point in the metabolism of $B$. subtilis and several other organisms. However, some organisms, like M. pneumoniae, live without a TCA cycle (Manolukas et al., 1988; Halbedel et al., 2007). Furthermore, the final MiniBacillus strain will only use glycolysis and the pentose-phosphate pathway to produce energy. Therefore, the main carbon source will be glucose (Reuß et al., 2016). To estimate the impact of the TCA cycle deletion and its possible effect on the MiniBacillus strain, a TCA cycle mutant in the wild type background should be created.

\subsubsection{The two citrate synthases of Bacillus subtilis}

In glycolysis, glucose is converted to pyruvate, which is used by the pyruvate dehydrogenase to produce acetyl-CoA. The initial step of acetyl-CoA into the TCA cycle is catalysed by the citrate synthase. Two citrate synthase genes are annotated in the genome of B. subtilis, citZ and citA. The genes and the transcription levels of two enzymes were already studied and compared (Jin and Sonenshein, 1994a). The enzyme CitZ was characterized as the major citrate synthase, since the deletion of citZ has a greater impact on growth than the deletion of citA (Zschiedrich, 2014). CitA seems to be a minor citrate synthase, but it is poorly characterized and has an unknown function in the cell. The co-transcribed gene citR seems to encode for a transcriptional repressor of citA. The effect of the deletion of the genes and the expression of citA under different conditions was already analysed (Zschiedrich, 2014). However, the role of CitA and also CitZ in the TCA cycle needs to be clarified to estimate which of the genes has to be deleted to construct a complete TCA cycle deletion strain.

The growth of the single and double deletion mutants of citZ, citA and citR in LB and Cmalate medium was analysed (Figure 15). In C-malate medium, the strains need a functional citrate synthase to produce citrate and glutamate, which is essential for the cell.All the single and double mutants grow similarly in the complex medium LB, which provides a lot of nutrients and amino acids. A deletion of these genes in the MiniBacillus strain would lead to no growth defect in the selected medium LB-glc. However, the strains with a deletion in citZ show a growth disadvantage in C-malate medium. 
A

LB medium

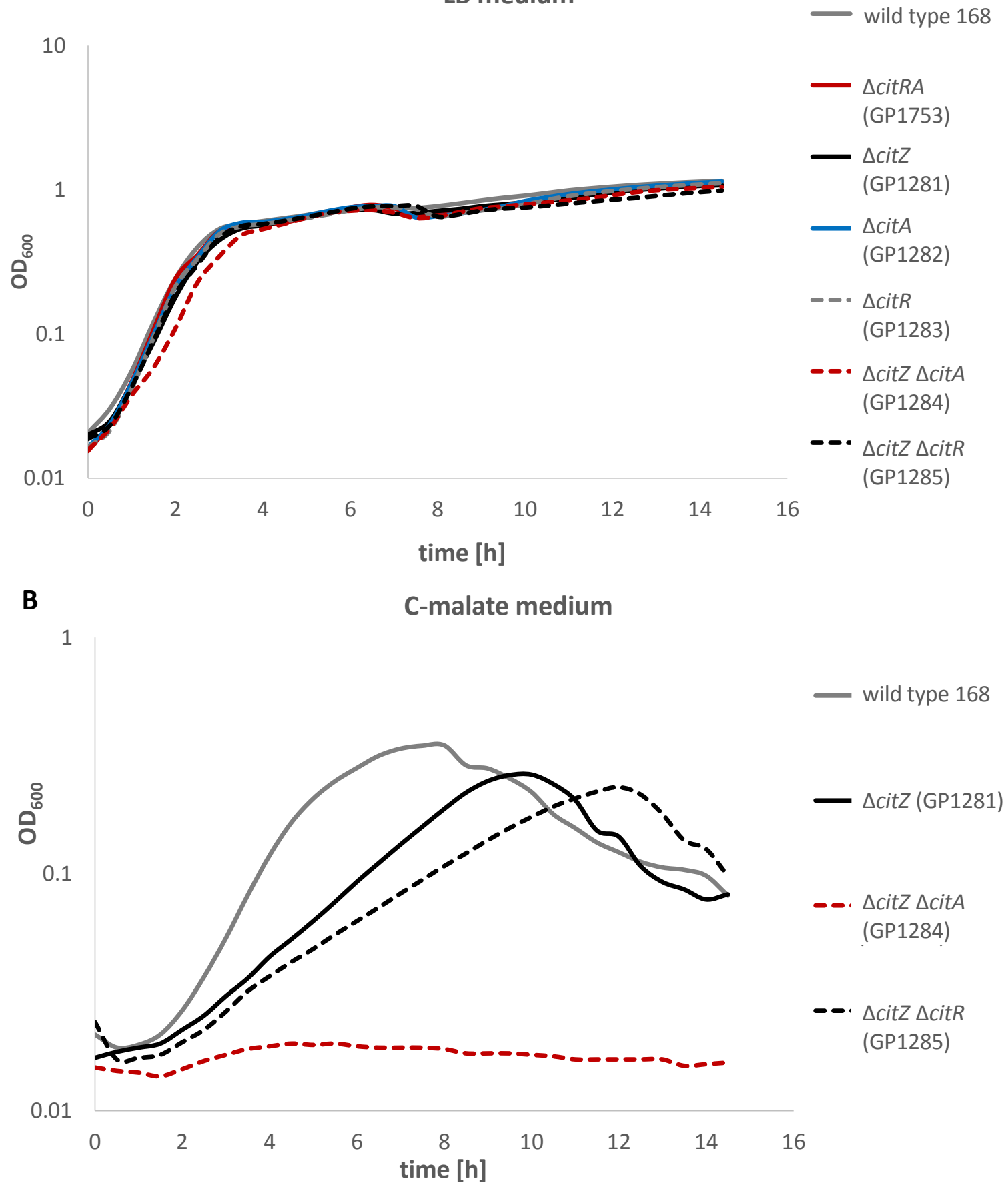

Figure 15: The growth of the citZ, citA and citR deletion mutants in LB medium (A) and C-malate medium (B). The precultures were prepared in LB medium and the growth was monitored at $37^{\circ} \mathrm{C}$.

The $\Delta c i t Z$ mutant grows slower than the wild type or the $\Delta c i t A$ mutant, which supports the theory, that CitZ is the major citrate synthase. The strains $\Delta c i t A, \Delta c i t R$ and $\triangle c i t R A$ grow like the wild type (data not shown). The double mutant $\Delta c i t z \Delta c i t A$ cannot grow at all in this medium. This indicates that CitA also functions as a citrate synthase and that CitZ and CitA are the only enzymes that can catalyse the reaction to citrate under these conditions. The growth defect is probably caused by the reduced glutamate production, which was already described before (Jin and Sonenshein, 1996). 
Interestingly, if citR is deleted in addition to citz, the growth is even worse than the growth of the $\Delta c i t Z$ mutant. In theory, in this strain the transcription of citA should be no longer repressed by CitR and so this strain should be able to produce more citrate. However, the mechanism of repression is poorly understood and it could be more complex which might lead to this observed growth disadvantage.

The activity of the major citrate synthase CitZ was already described before (Jin and Sonenshein, 1996) and a first attempt to compare the activities of the two citrate synthases in vitro was performed by Zschiedrich (2014). However, the verification of these results and the determination of the $\mathrm{Km}$ and $\mathrm{Vmax}$ values will further characterize the activities of both enzymes. Therefore, the $\mathrm{N}$-terminal Strep-tagged proteins were each overexpressed in $E$. coli from the plasmids pGP2515 (Strep-CitZ) and pGP2516 (Strep-CitA). The cells were disrupted with the French press and the proteins were purified via Strep-Tactin as described (chapter 2.2.7). The SDS page shows the expected sizes for the purified CitZ and CitA proteins (Figure 16).

A

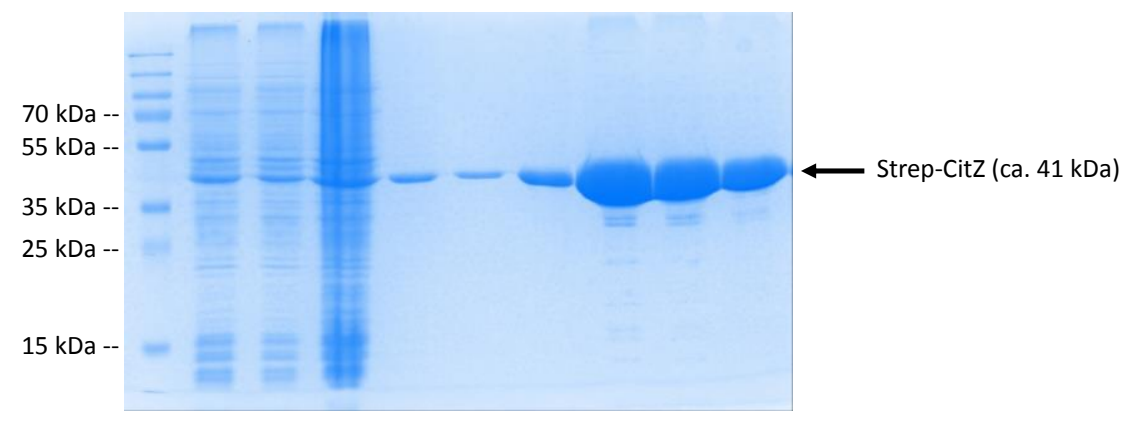

B

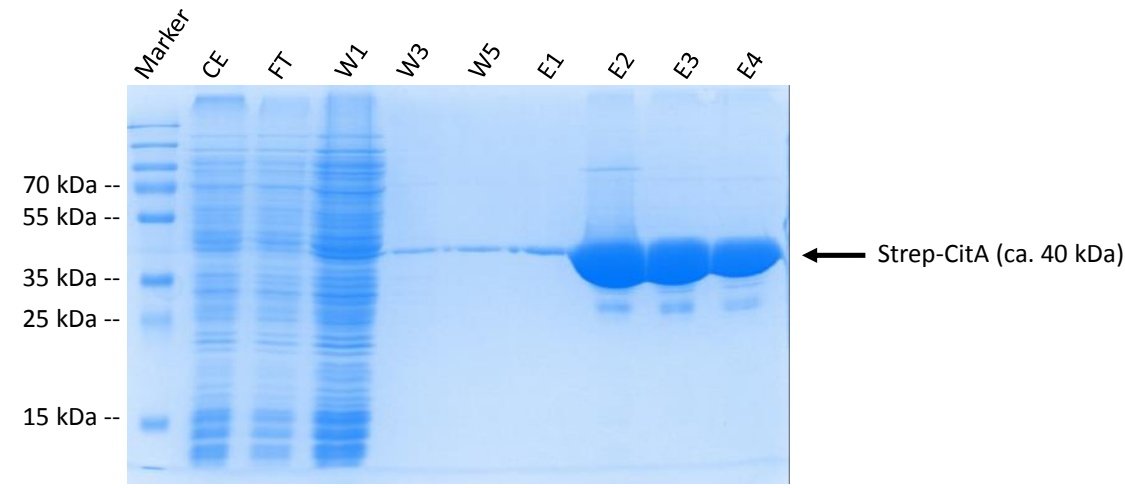

Figure 16: The SDS gel of the citrate synthase protein purifications. A The major citrate synthase CitZ was eluted with the size of about $41 \mathrm{kDa}$. B The strep-tagged CitA protein was purified with a size of about $40 \mathrm{kDa}$. ( $\mathrm{CE}=$ crude extract, $\mathrm{FT}=$ flow through, $\mathrm{W}=$ wash fraction, $\mathrm{E}=$ elution fraction)

The amount of purified protein was measured and the enzymes were used in a citrate synthase activity assay. The citrate synthase converts oxaloacetate and acetyl-CoA to citrate and coenzyme 
A. In this assay the released coenzyme $A$ can react with the added ellmanns reagent, 5,5'-dithiobis (2-nitrobenzoic acid) (DTNB) (Ellman, 1959). The resulting product 2-nitro-5-chlorobenzaldehyde (TNB) can be measured spectrophotometrically at a wavelength of $412 \mathrm{~nm}$. The assay was performed with each enzyme as described in chapter 2.2.7. The change in absorption over time was measured with a plate reader at $25^{\circ} \mathrm{C}$ and a blank measurement without enzyme was subtracted (Figure 17).

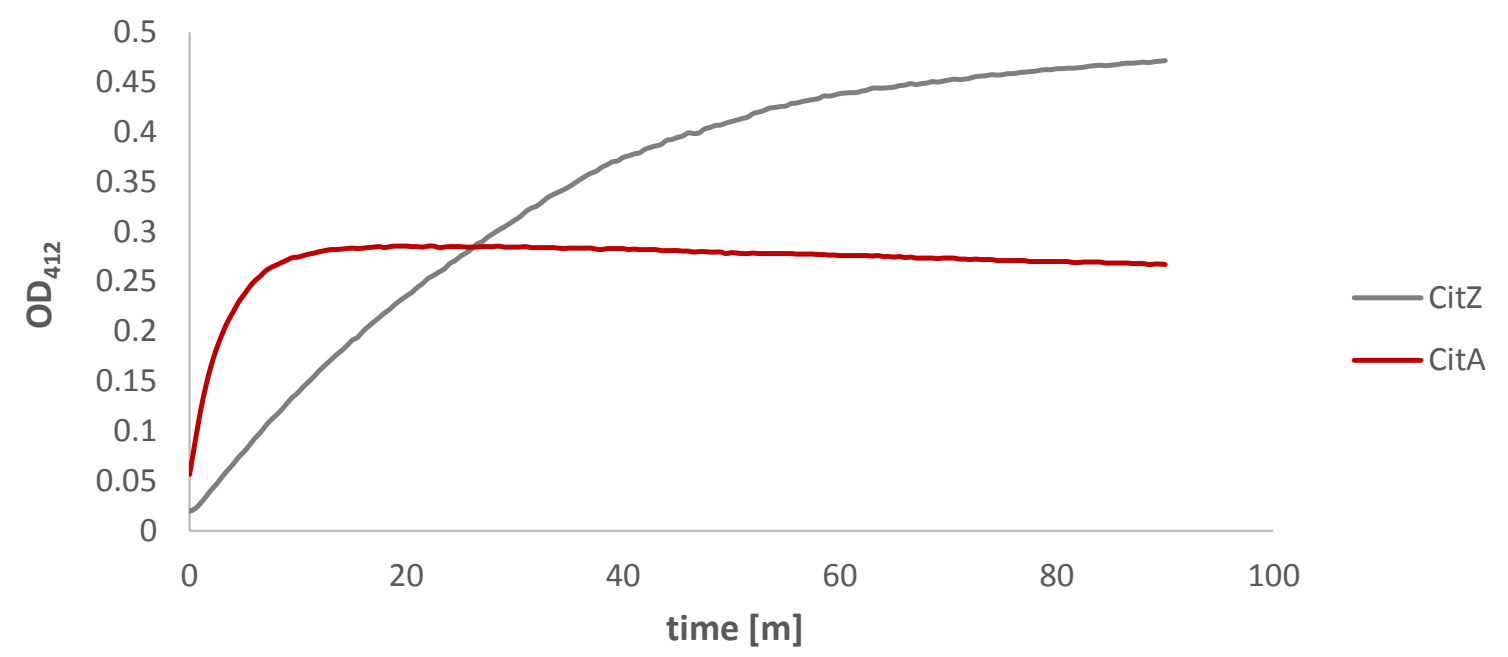

Figure 17: The activity assay of the two citrate synthases. The reaction was measured spectophotometrically at $412 \mathrm{~nm}$ and at $25^{\circ} \mathrm{C}$.

The activity assay of the two enzymes shows that both harbour citrate synthase activity, since the produced coenzyme A could react with the DTNB. Interestingly, the previously described minor citrate synthase CitA seems to catalyse the reaction faster. However, the major citrate synthase CitZ seems to produce almost the double amount of citrate in comparison to CitA.

Table 6: The $K_{m}$ and $V_{\max }$ values for the two citrate synthases CitZ and CitA for the substrates oxaloacetate and acetyl-CoA.

\begin{tabular}{|c|c|c|c|}
\hline \multirow{2}{*}{$K_{m}$} & Substrate & CitZ & CitA \\
\hline & Acetyl-CoA & $164.41 \mu \mathrm{M}$ & $14.24 \mu \mathrm{M}$ \\
\cline { 2 - 4 } & Oxaloacetate & $64.14 \mu \mathrm{M}$ & $53.95 \mu \mathrm{M}$ \\
\hline \multirow{2}{*}{$\mathbf{V}_{\max }$} & Acetyl-CoA & $26.7 \mathrm{mM} / \mathrm{min}$ & $3.46 \mathrm{mM} / \mathrm{min}$ \\
& Oxaloacetate & $9.71 \mathrm{mM} / \mathrm{min}$ & $10.28 \mathrm{mM} / \mathrm{min}$ \\
\hline
\end{tabular}

The Km and Vmax values were determined for each of the substrates, oxaloacetate and acetyl-CoA. Several measurements were performed with one substrate in a constant concentration and the 
substrate of interest in varying concentrations $(0.03-0.45 \mathrm{mM})$. The $\mathrm{Km}$ and Vmax values for the two citrate synthases and both substrates were calculated as described in chapter 2.2.7. and they are listed in Table 6.

The higher Km values of CitZ for both substrates indicate a weaker binding of the enzyme to the substrates in comparison to CitA. So CitZ needs a higher concentration of substrate to reach Vmax. These results suggest that both proteins can be active as citrate synthases and should therefore be deleted to obtain a clean TCA cycle deletion mutant. Nevertheless, CitZ seems to be the major citrate synthase. Additionally, the deletion of the repressor CitR will also be included, since its physiological role is still unknown, but it could be linked to the TCA cycle.

\subsubsection{The deletion of the TCA cycle}

For the deletion of the complete TCA cycle, the single genes for the involved enzymes have to be deleted (Table 7).

Table 7: The genes and operons encoding for enzymes that are involved in the TCA cycle.

\begin{tabular}{|l|l|}
\hline Genes & Protein function \\
\hline citZ & Major citrate synthase \\
\hline icd & Isocitrate dehydrogenase \\
\hline mdh & Malate dehydrogenase \\
\hline citA & Minor citrate synthase \\
\hline citB & Transcriptional repressor of citA \\
\hline odhA & Aconitase \\
\hline odhB & 2-oxoglutarate dehydrogenase (E1 subunit) \\
\hline sucC & Succinyl-CoA synthetase (beta subunit) \\
\hline sucD & Succinyl-CoA synthetase (alpha subunit) \\
\hline sdhC & Succinate dehydrogenase (cytochrome b558 subunit) \\
\hline sdhA & Succinate dehydrogenase (flavoprotein subunit) \\
\hline sdhB & Succinate dehydrogenase \\
\hline citG & Fumarase \\
\hline
\end{tabular}

The first deletion attempts were performed in the wild type strain 168 and every single gene or operon should be exchanged step by step with a resistance cassette. However, the strain could not 
be finished, since just a few deletions lead to the loss of genetic competence. In the MiniBacillus strain, the mannitol inducible comKS system is used to increase the transformation efficiency. An additional copy of the competence genes comK and comS is introduced into the chromosome and the addition of mannitol to the medium induces the $m$ t/A promoter in front of the genes and thereby genetic competence. It could be shown, that the competence increases around 7-fold in comparison to the wild type (Rahmer et al., 2015). Therefore, the next deletion attempt was performed in the background of a strain with the inducible comKS system (GP2973). This strain also harbours the xylose-inducible Cre recombinase from P1 bacteriophage, integrated into sacA. This enzyme is used in the Cre-lox system to remove the resistance cassette from a deletion, by recombining the additionally introduced lox sites. Only a small scar, the lox72 site is left behind. This method is necessary, since the available resistance cassettes are not sufficient to enable the deletion of all genes. The stepwise created strains of the TCA cycle deletion are listed in Table 8.

Table 8: The strains of the stepwise TCA cycle deletion

\begin{tabular}{|c|c|}
\hline Strain & Genotype \\
\hline GP2973 & $\operatorname{trpC2}$ yvcA-P $P_{m t / A}-c o m K S-m / s-h i s /$ sacA::(phl-Pxy/A-cre) \\
\hline GP2974 & GP2973 $\Delta$ citZ-icd-mdh::lox72 \\
\hline GP2975 & GP2973 $\Delta$ citZ-icd-mdh::lox72 $\Delta$ sucCD::tet \\
\hline GP3024 & GP2973 $\Delta$ citZ-icd-mdh::lox72 $\Delta$ sucCD::tet $\Delta$ sdhCAB::Iox72 \\
\hline GP3025 & GP2973 $\Delta$ citZ-icd-mdh::lox72 $\Delta$ sucCD::tet $\Delta$ sdhCAB::lox72 $\Delta$ citG::spec \\
\hline GP3026 & GP2973 $\Delta$ citZ-icd-mdh::lox72 $\Delta$ sucCD::tet $\Delta$ sdhCAB::lox72 $\Delta$ citG::spec $\Delta$ odhAB::cat \\
\hline GP3027 & $\begin{array}{l}\text { GP2973 } \Delta \text { citZ-icd-mdh::lox72 } \Delta \text { sucCD::tet } \triangle \text { sdhCAB::lox72 } \Delta \text { citG::spec } \triangle \text { odhAB::cat } \\
\Delta \text { citB::lox72 }\end{array}$ \\
\hline GP3028 & $\begin{array}{l}\text { GP2973 } \Delta \text { citZ-icd-mdh::lox72 } \Delta \text { sucCD::tet } \triangle \text { sdhCAB::lox72 } \Delta \text { citG::spec } \triangle \text { odhAB::cat } \\
\Delta \text { citB::lox72 } \Delta \text { citRA::lox79 }\end{array}$ \\
\hline
\end{tabular}

The first step was to delete the genes citZ, icd and $m d h$ that are encoded in one operon. The operon was exchanged with a kanamycin resistance cassette, flanked by the two lox sites lox71 and lox66. This cassette was then removed by the Cre recombinase, which was expressed upon the addition of xylose, leaving the lox72 site behind. The next step was to delete the succinyl-CoA synthetase encoded by sucCD operon by the introduction of a tetracycline resistance cassette. For the deletion 
of the succinate dehydrogenase encoded by the $s d h C A B$ operon, the Cre-lox system was used and the gene citG (fumarase) was deleted with a spectinomycin resistance cassette. The 2-oxoglutarate dehydrogenase consists of three subunits OdhAB and PdhD. However, the deletion of odhAB is sufficient to delete the pathway. The protein PdhD is additionally involved in the conversion of pyruvate to acetyl-CoA as a part of the pyruvate dehydrogenase complex. Furthermore, a $\Delta p d h D$ strain is unable to grow on glucose as the single carbon source which makes this gene essential for the MiniBacillus strain (Gao et al., 2002). The operon odhAB was deleted by the replacement with a chloramphenicol resistance cassette. Afterwards, citB and citRA were one after another deleted with the Cre-lox system. The final strain (GP3028) has a deletion in all genes encoding for enzymes involved in the TCA cycle.

\subsubsection{The phenotype of the TCA cycle deletion strain}

Although the competence needed to be improved, a B. subtilis strain without TCA cycle could be constructed, which means that $B$. subtilis can live without a TCA cycle. Growth experiments were performed to investigate the impact of the TCA cycle deletion on the growth (Figure 18). The growth of the TCA cycle deletion mutant was compared to the wild type and the parental strain GP2973. All three strains show a good growth in LB-glc medium. The TCA cycle mutant does not seem to have a growth disadvantage, which leads to the assumption that the deletion of the TCA cycle will not lead to a growth disadvantage in the MiniBacillus strain. However, the growth test in C minimal medium with only glucose shows that the lack of the TCA cycle leads to a growth defect under these conditions. The reason for this might be that the cells cannot produce glutamate anymore, which is essential for the organism. The addition of glutamate to the medium (CE-glc) leads then again to a better growth of the TCA cycle deletion strain. This supports also the assumption that the strain is auxotrophic for glutamate.

Furthermore, the strain lacking both citrate synthases was also unable to grow on minimal medium without glutamate, as described above. However, all other amino acids need to be synthesized in the CE-glc minimal medium, since they cannot be imported from the medium. Some are derived from glycolysis or the added glutamate, but aspartate is produced from oxaloacetate, an intermediate of the TCA cycle. But since the strain can grow without additional aspartate in the medium, aspartate must be synthesized. The pyruvate carboxylase PycA catalyses the reaction from pyruvate to oxaloacetate, which can then be converted to aspartate by the aspartate transaminase AspB (Figure 19). 
A

LB-glc medium

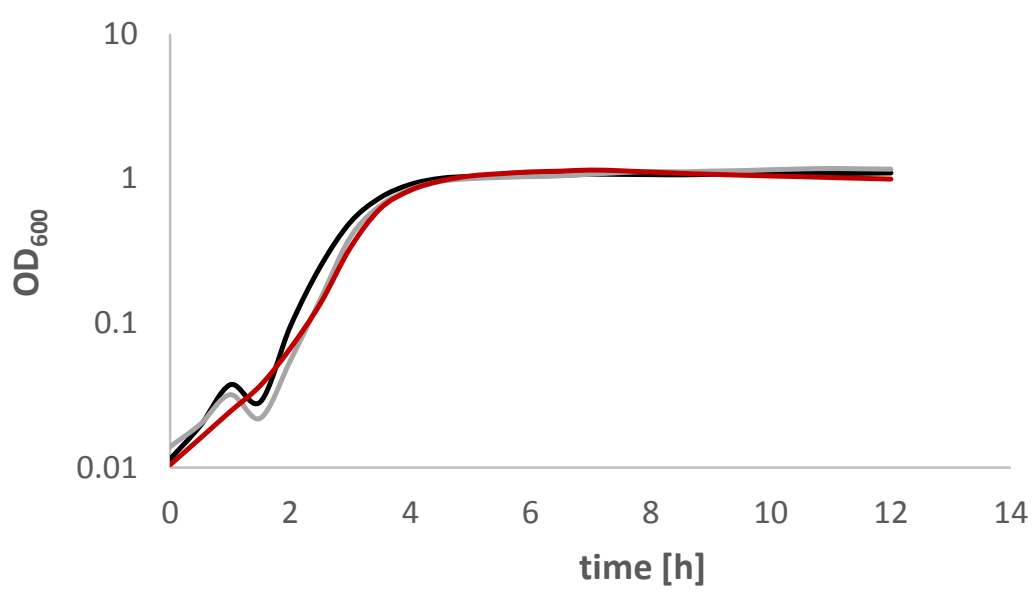

— wild type 168

_ GP2973

$-\triangle T$ TCA cycle

(GP3028)

B

C-glc medium

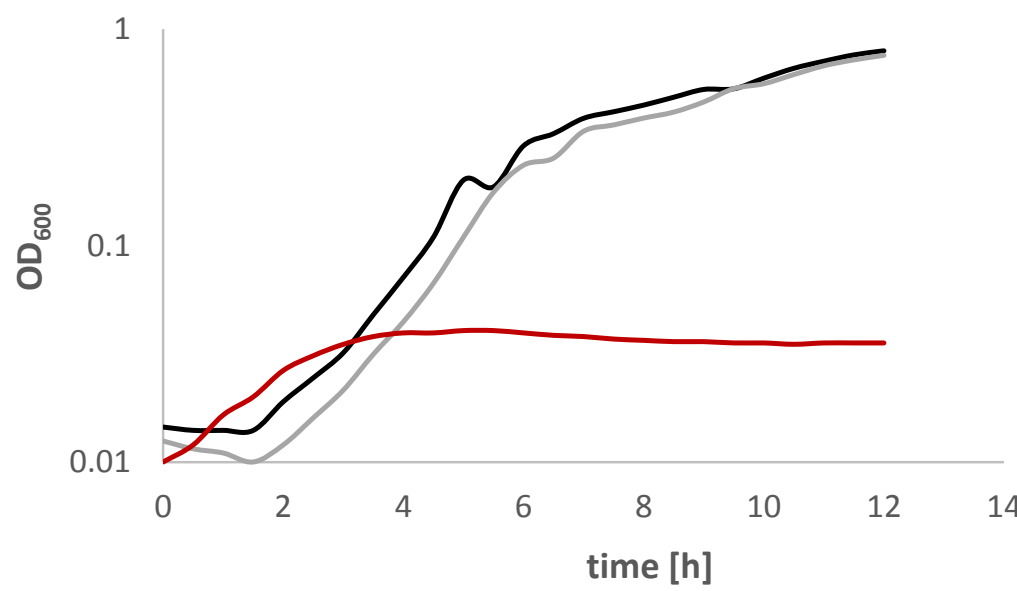

— wild type 168

- GP2973

$-\triangle \mathrm{TCA}$ cycle

(GP3028)

C

CE-glc medium

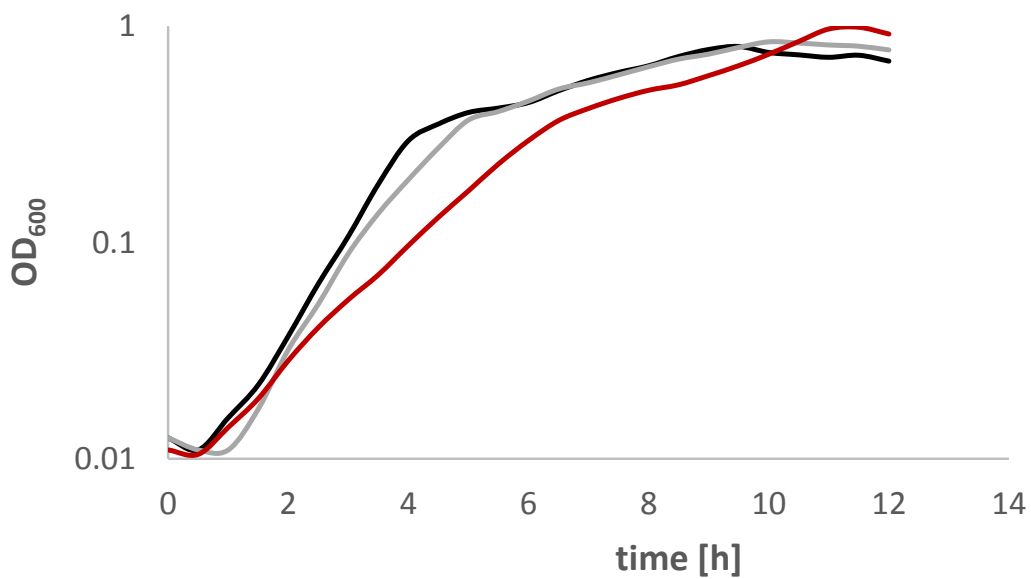

— wild type 168

— GP2973

$\longrightarrow \triangle \mathrm{TCA}$ cycle (GP3028)

Figure 18: The growth curves for the TCA cycle deletion strain (GP3028) in comparison to the wild type strain 168 and the parental strain GP2973. Precultures were prepared in LB medium. The strains were grown at $37^{\circ} \mathrm{C}$ in LB-glc medium (A), C-glc medium (B) and CE-glc medium (C). 


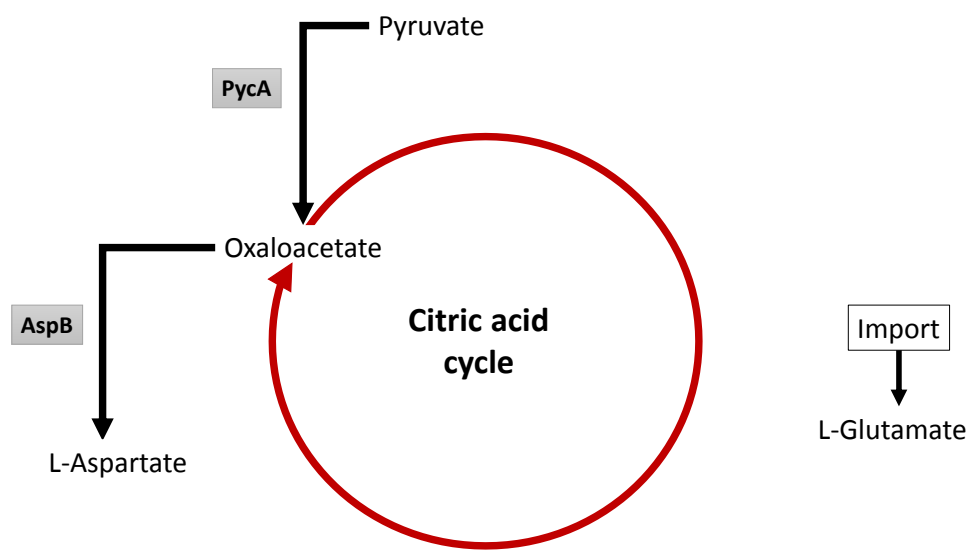

Figure 19: The impact of the TCA cycle deletion on the amino acid metabolism. The deletion of the TCA cycle causes an auxotrophy for glutamate. However, pyruvate can still be converted to oxaloacetate by PycA which is then converted to aspartate by AspB.

Since the competence was reduced in the first attempt to delete the TCA cycle, the competence of the final strain and the intermediate steps were tested. The protocol for the preparation of competente cells with the comKS system was used as described (chapter 2.2.5.), but the competent cells were each diluted to an $\mathrm{OD}_{600}$ of 0.5 and $1 \mathrm{ml}$ of them were transformed with $50 \mathrm{ng}$ pAC7 plasmid DNA (Figure 20).

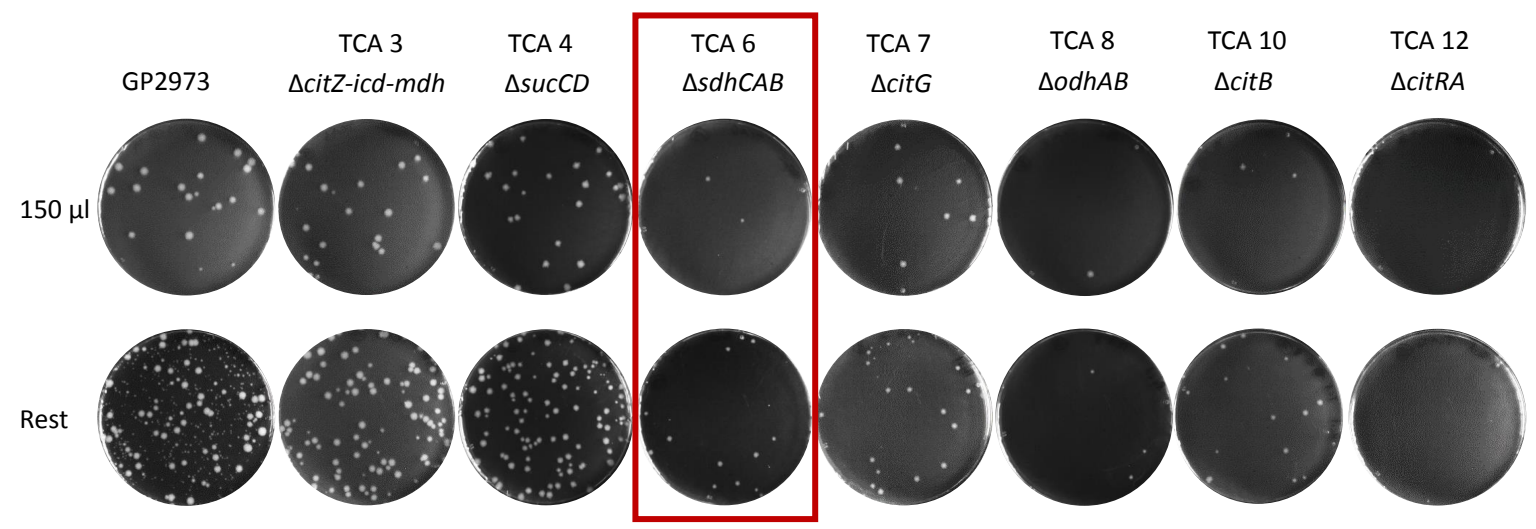

Figure 20: The competence test of the final and the intermediate strains of the TCA cycle deletion mutant. The strains were diluted to the same $\mathrm{OD}_{600}$ of 0.5 and transformed with $50 \mathrm{ng}$ pAC7 plasmid. The cells were finally plated on LB-glc plates with kanamycin.

The competence test shows that the TCA cycle deletion strain has a highly reduced competence in comparison to the parental strain GP2973. However, some transformation colonies are still visible. Interestingly, the deletion of $s d h C A B$ seems to have the greatest impact on the competence. To investigate, if the deletion of $s d h C A B$ or the combination of the different deletions is causing this phenotype, a strain was created in the GP2973 background with a deletion of only sdhCAB and tested for the competence (Figure 21).

The competence of the strain with the single $s d h C A B$ deletion is highly reduced in comparison to the parental strain GP2973. $50 \mathrm{ng}$ of the plasmid pAC7 was added to $1 \mathrm{ml}$ of cells 
$\left(\mathrm{OD}_{600}\right.$ of 0.3$)$. This leads to a calculated transformation efficienty of 1,720 transformants per $\mu \mathrm{g}$ DNA of GP2973 and 280 transformants per $\mu \mathrm{g}$ DNA of GP2973 with the deletion of $s d h C A B$. This indicates that this operon is necessary to maintain the competence.

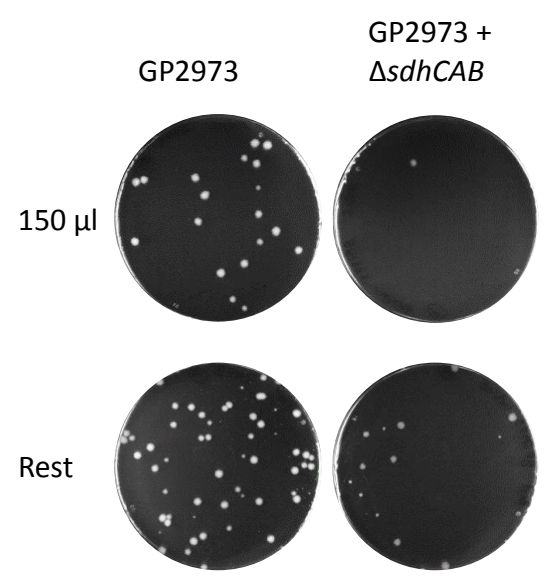

Figure 21: The competence test of the $s d h C A B$ single deletion strain. The strains were diluted to the same $\mathrm{OD}_{600}$ of 0.3 and mixed with $50 \mathrm{ng}$ of the plasmid pAC7. After another inoculation step for 1 hour, the cells were plated onto LB-glc plates with kanamycin.

The protein complex SdhCAB is not only involved in the TCA cycle, but it is also involved in the respiratory chain. SdhC is the membrane anchor of the complex and is involved in the electron transfer to menaquinone, since it is a part of cytochrome b558 (Hederstedt and Rutberg, 1983; Baureder and Hederstedt, 2011). The deletion of genes involved in the respiratory chain was already observed to lead to a reduction or loss of competence, e.g for the NADH dehydrogenase Ndh (Koo et al., 2017). However, the loss of competence in the final TCA cycle deletion strain could also result from the combination of all mutations, since the $s d h C A B$ single deletion strain seems to be still more competent than the final TCA cycle deletion strain. Since the comKS system is also used in the MiniBacillus strain, the deletion of the TCA cycle would also lead to a reduced competence. This would be detrimental for the project, since the cells need to be transformed with a plasmid in the deletion system. The reduction of genetic competence would complicate the deletion process.

Some deletion mutants of TCA cycle genes are described to have a defect in sporulation. The icd deletion mutant e.g. exhibit a reduced ability to sporulate. The reason fo this was the accumulation of citrate and isocitrate. However, the additional deletion of the citrate synthase citz seems to be able to counteract this effect (Matsuno et al., 1999). In a deletion of the complete TCA cycle, no intermediates can accumulate. Furthermore, the effect of the comKS system on the sporulation was never investigated before. This might be interesting since the amount of the phosphorylated sporulation master regulator SpoOA influences the amount of ComK in the cell and thereby also competence (Fujita et al., 2005). The sporulation of the TCA cycle deletion strain was tested as described in chapter 2.2.9. (Figure 22). 


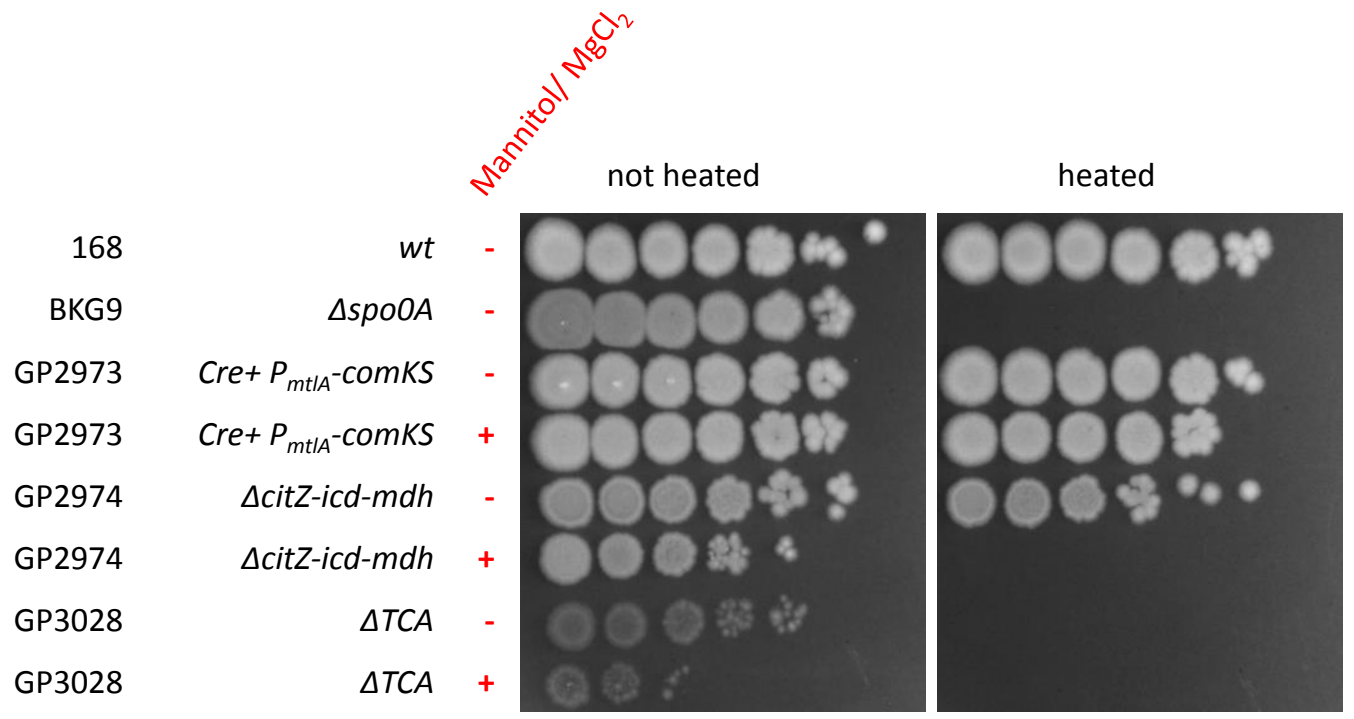

Figure 22: The sporulation test of the TCA cycle mutant in SP medium. The wild type strain 168 serves as a positive control and the BKG9 $(\triangle s p o O A)$ as a negative control for sporulation. The strains with the inducible comKS system were tested with and without the inducer mannitol. The cells were plated on LB plates.

In the sporulation assay, the number of spores formed in a SP culture from different strains are compared. One sample of each strain was heated at to remove all living cells, so that only the spores survive. The wild type strain 168 formed many spores which were able to grow again on the LB plates after the heating step. In contrast, the $\triangle$ spoOA mutant is not able to form spores at all, since spoOA is the regulator of a phosphorelay to initiate sporulation (Burbulys et al., 1991). The strain GP2973, with the comKS system and the integrated cre recombinase, forms the same number of spores as the wild type. In this case, the addition of mannitol and $\mathrm{MgCl}_{2}$ for the induction of the comKS system, has almost no effect on the spore formation. However, the $\Delta c i t Z$-icd-mdh operon mutant has a reduced number of spores in SP medium without mannitol and $\mathrm{MgCl}_{2}$. This was already reported previously for the single deletion mutants $\Delta c i t Z$ and $\Delta i c d$ (Jin and Sonenshein, 1994b; Jin et al., 1997). If the comKS system is induced by the two components, there is no sporulation detectable anymore. This indicates an influence of the ComK level on sporulation. Additionally, the growth of the not heated samples is also reduced in comparison to the sample with only SP medium. The TCA cycle deletion strain GP3028 shows very weak growth in both media, especially with the addition of mannitol and $\mathrm{MgCl}_{2}$. Additionally, the strain cannot form spores anymore. The reason for the loss of sporulation was often explained by the accumulation of intermediates of the TCA cycle. Especially the accumulation of citrate seemed to have a great impact, since citrate builds a complex with divalent cations which are required for the Spo0A phosphorelay (Craig et al., 1997; Matsuno et al., 1999). However, in a TCA cycle deletion mutant, no intermediates accumulate, since the complete pathway is deleted. But as previously described, there are still pathways that produce intermediates that are normally used by the TCA cycle, like e.g. oxaloacetate by the pyruvate carboxylase PycA. 
A

$\triangle T$ TA cycle

(GP3028)
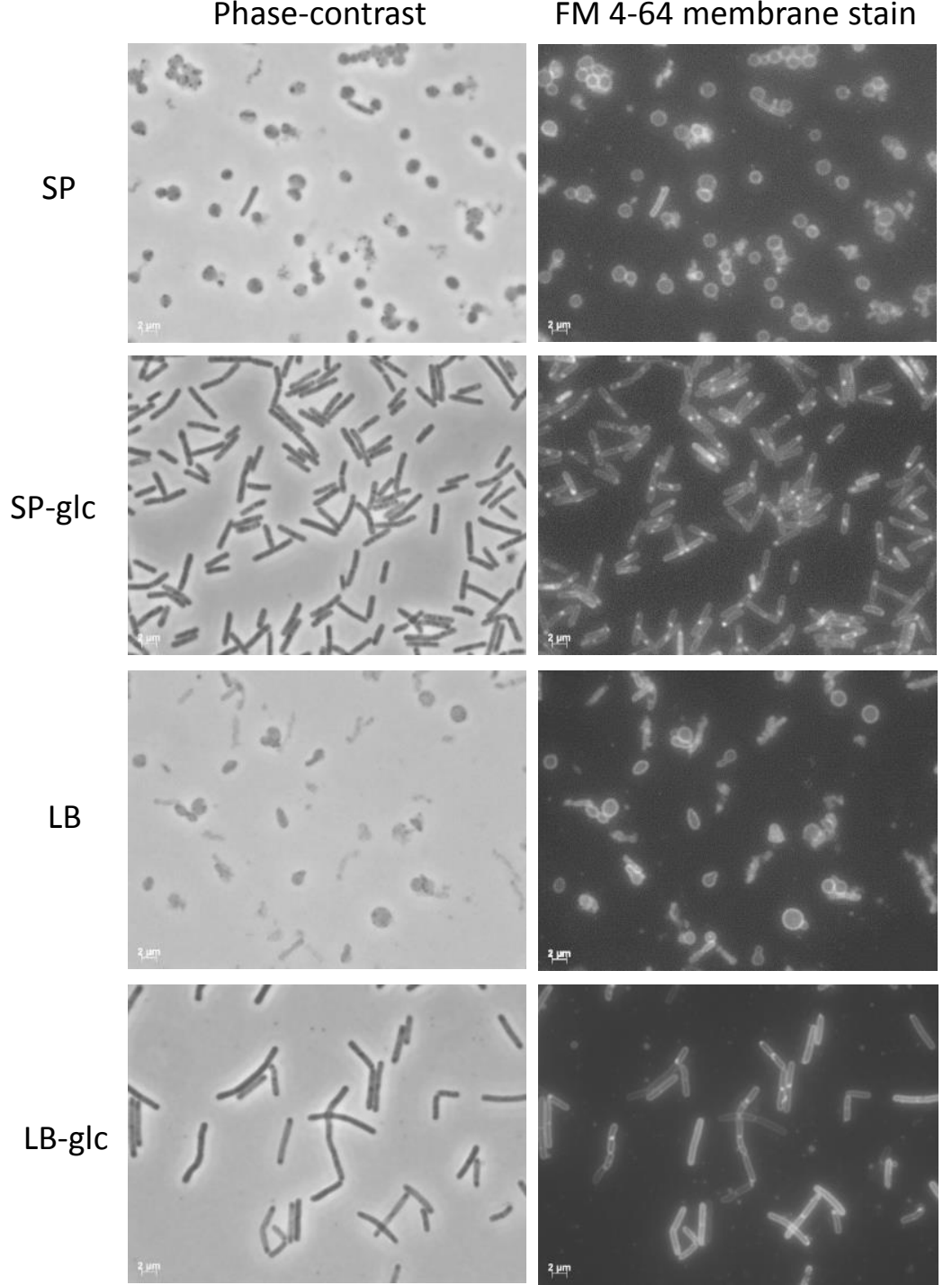

B

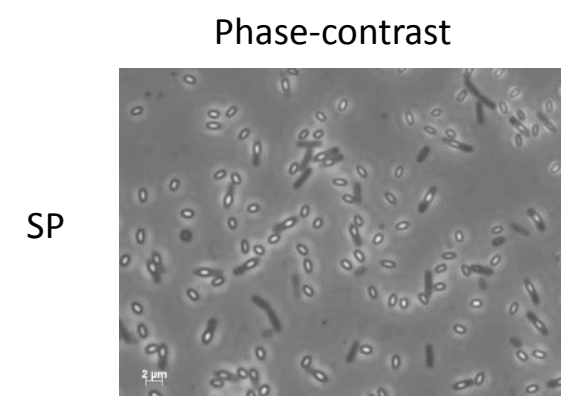

FM 4-64 membrane stain

Wild type 168
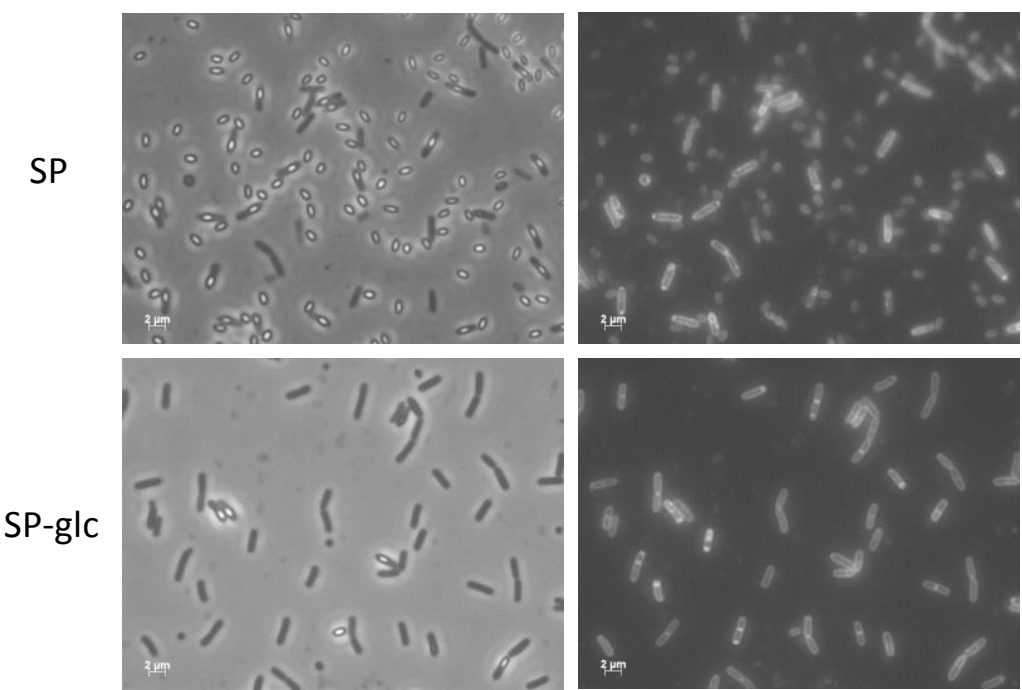

Figure 23: The microscopy of the TCA cycle deletion strain (GP3028) grown in LB and SP medium with and without $\mathbf{0 . 5 \%}$ glucose (A). The wild type 168 was grown in SP and SP-glc medium as a control (B). 
Finally, the TCA cycle deletion strain was also analysed at the single cell level under the microscope. The strain was grown in LB and SP medium with the addition of $0.5 \%$ glucose or without. The cells were additionally stained with the FM4-64 membrane stain (Figure 23). The TCA cycle deletion cells from the SP-glc cultures are rod-shaped, like the wild type cells of the control. However, they are not able to form spores what confirms the results from the sporulation assay. The wild type 168 forms spores in SP medium. In contrast, almost all cells of the TCA cycle deletion strain from the SP medium without additional carbon source are forming round structures. In the FM 4-64 stain, the lipids of the membrane are stained and can therefore be observed surrounding the round structures. This effect can also be seen in LB cultures without additional glucose.

These round structures can always be observed, if the peptidoglycan cell wall, is missing, in either protoplasts or L-form cells. L-form cells are lacking the peptidoglycan completely and are still able to grow.

Phase-contrast

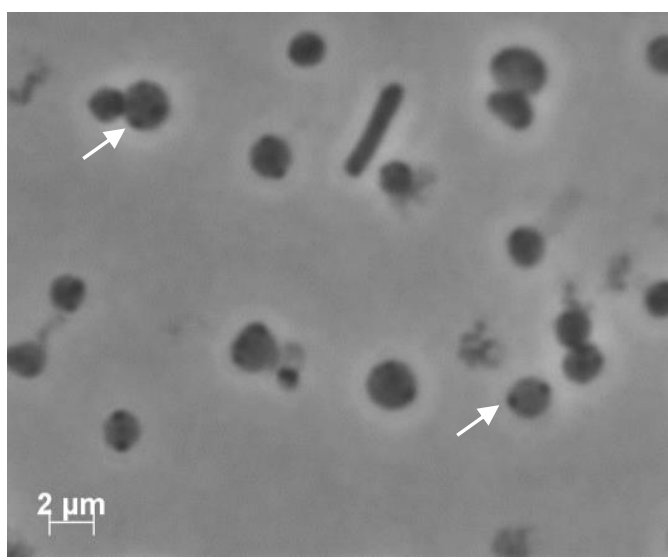

FM 4-64 membrane stain

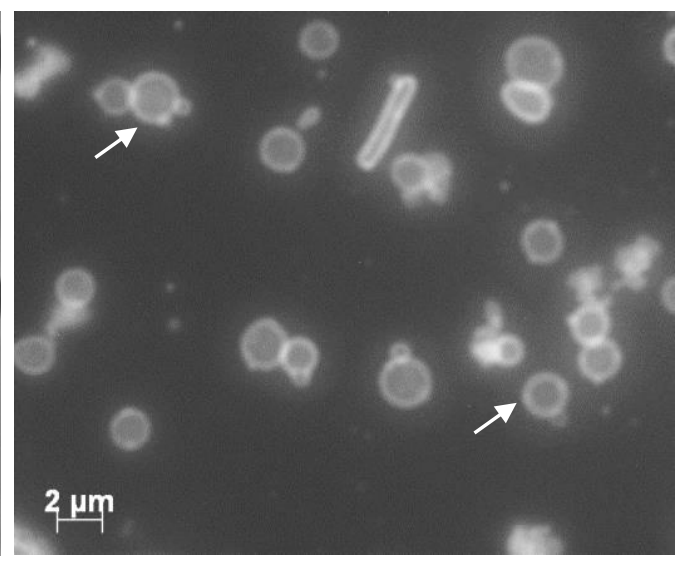

Figure 24: The spherical cells grown in SP medium without additional carbon source. The arrows indicate small darker dots on the surface of the cells.

On the magnified cells in Figure 24, small black dots can be observed on the surface. They cannot be found in the image with the membrane stain, which means those are no clusters of lipids. However, since L-form cells have often a block in peptidoglycan synthesis, theses dots could be leftover peptidoglycan, since the spherical cells are not as dark as the single rod-shaped cell in the Figure. Since the missing additional carbon source seems to be the reason for the appearance of the spherical cells, this could mean that the TCA cycle deletion strain is in this case not able to produce enough peptidoglycan to sustain the rod-shaped cell morphology. For the production of peptidoglycan, fructose-phosphate and phosphoenolpyruvate from glycolysis are used, as well as acetyl-CoA, which is produced from pyruvate (Daniel and Errington, 1993). If additional glucose is added, the cells can produce enough intermediates from glycolysis to form the peptidoglycan cell wall and the rod-shaped form. However, it still needs to be elucidated if these cells are protoplasts or L-form cells. L-form cells are able to divide and therefore also to grow in a culture. This was tested 
with a preculture of the TCA cycle deletion strain in SP medium. From this culture, different cultures were inoculated, and growth was monitored (Figure 25).
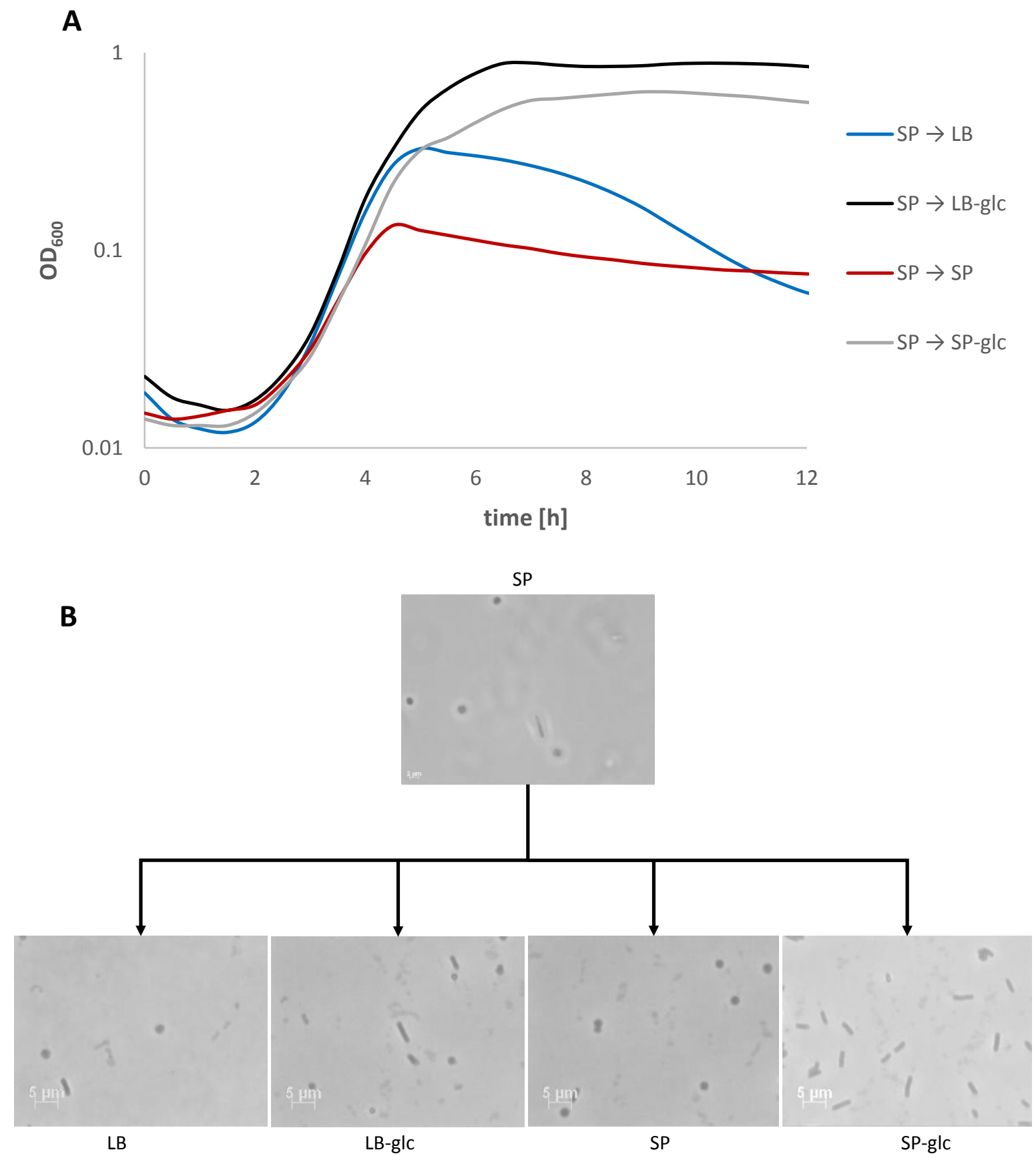

Figure 25: The growth of the TCA cycle deletion strain from a SP preculture in different media. A The growth of the strain in LB, LB-glc, SP and SP-glc medium, inoculated from the same SP preculture. B The cell morphology of the different cultures after 12 hours.

Almost all cells from the preculture showed the spherical phenotype, that was observed before. Only a few cells are still rod-shaped (Figure 25 B). The main cultures that were inoculated from this preculture showed first the same growth rate. However, the final $\mathrm{OD}_{600}$ of the cultures differ. The LB medium seemed to be better for the growth than the SP medium, since the cells from the LB 
medium reached a higher $\mathrm{OD}_{600}$. However, the addition of glucose to either LB or SP medium caused an even higher final $\mathrm{OD}_{600}$. For the decision, if the spherical cells are still able to divide and grow, the growth curve in SP is the most interesting. It seemed that the cells are still able to grow, since the $\mathrm{OD}_{600}$ rises. However, there were still rod-shaped cells in the SP preculture which could have caused the raised $\mathrm{OD}_{600}$. But since this is not a perfectly osmotically balanced medium, it could be possible that the spherical cells are able to grow, but some are burst because of the osmotic imbalance. Deletion mutants with reduced peptidoglycan synthesis are capable to change into the L-form growth. If e.g. the peptidoglycan precursor pathway is inhibited by different antibiotics, the cells can switch into the L-form state (Mercier et al., 2014). Furthermore, the mutations or repression of genes involved in the respiratory chain are also capable of inducing L-form cells. This was observed for the, already previously mentioned, NADH dehydrogenase Ndh (Kawai et al., 2015). Since the succinate dehydrogenase complex SdhCAB is also involved in the respiratory chain, the L-form state could be similarly triggered in the TCA cycle deletion strain. These facts all may lead to the assumption that the TCA cycle deletion strain forms L-form cells instead of protoplasts.

To conclude, the TCA cycle deletion would probably lead to no effect on the growth of the MiniBacillus strain in LB-glc medium. However, since the MiniBacillus strain uses the same comKS system to induce the competence, the TCA cycle deletion will lead to strong decrease of the competence. This would be a drawback for the project. Furthermore, the deletion of the TCA cycle might drastically change the cell morphology. Although, the MiniBacillus strain is already not able to sporulate, the peptidoglycan synthesis will be hindered, and this might lead to the formation of L-form cells, if no additional carbon source is added. However, the effect on the MiniBacillus strain could be different in comparison to the effect on the wild type strain 168 , since over $40 \%$ of the genome is already deleted.

\subsection{The identification of serine transporters in Bacillus subtilis}

The amino acid transport is interesting for the MiniBacillus project, since the uptake of amino acids requires less genes than the biosynthesis pathways. However, in B. subtilis some amino acids have no characterized importer, e.g. tyrosine. This means the biosynthesis pathways remains in the blueprint of a minimal cell.

\subsubsection{The serine importer $\mathrm{YbeC}$}

For the identification of potential amino acid transporters, the database SubtiWiki was searched for candidates that show similarities to known transporters (Michna et al., 2016). Eight different candidates were chosen for a screening approach (Table 9). 
Table 9: The putative amino acid importers, that were chosen from SubtiWiki database.

\begin{tabular}{|l|l|l|l|}
\hline Gene & Product & Protein length & Deletion strain \\
\hline aapA & Amino acid permease & 459 aa & GP2377 \\
\hline steT & Serine/threonine exchanger transporter & 438 aa & GP2378 \\
\hline yfnA (mtrA) & Methylthioribose transporter & 461 aa & GP2379 \\
\hline ytnA & Unknown (similar to proline permease) & 463 aa & GP1885 \\
\hline ybeC & Unknown & 539 aa & GP1886 \\
\hline alsT & Putative glutamine transporter & 465 aa & GP1888 \\
\hline yveA & Similar to aspartate/ glutamate transporter & 520 aa & GP2385 \\
\hline yodF & Unknown & 496 aa & GP1887 \\
\hline
\end{tabular}

The protein SteT shows similarity to amino acid transporters from humans and it was shown to have a function in the exchange of serine and threonine in proteoliposomes (Reig et al., 2007). To elucidate its function directly in B. subtilis, this gene was also chosen for this screening approach. Furthermore, the gene $y f n A$ (now: $m t r A$ ) was also annotated as being similar to steT and therefore also chosen in this experiment (Reig et al., 2007). However, a recent publication of Borriss et al. (2018) stated, that MtrA is involved in the uptake of methylthioribose.

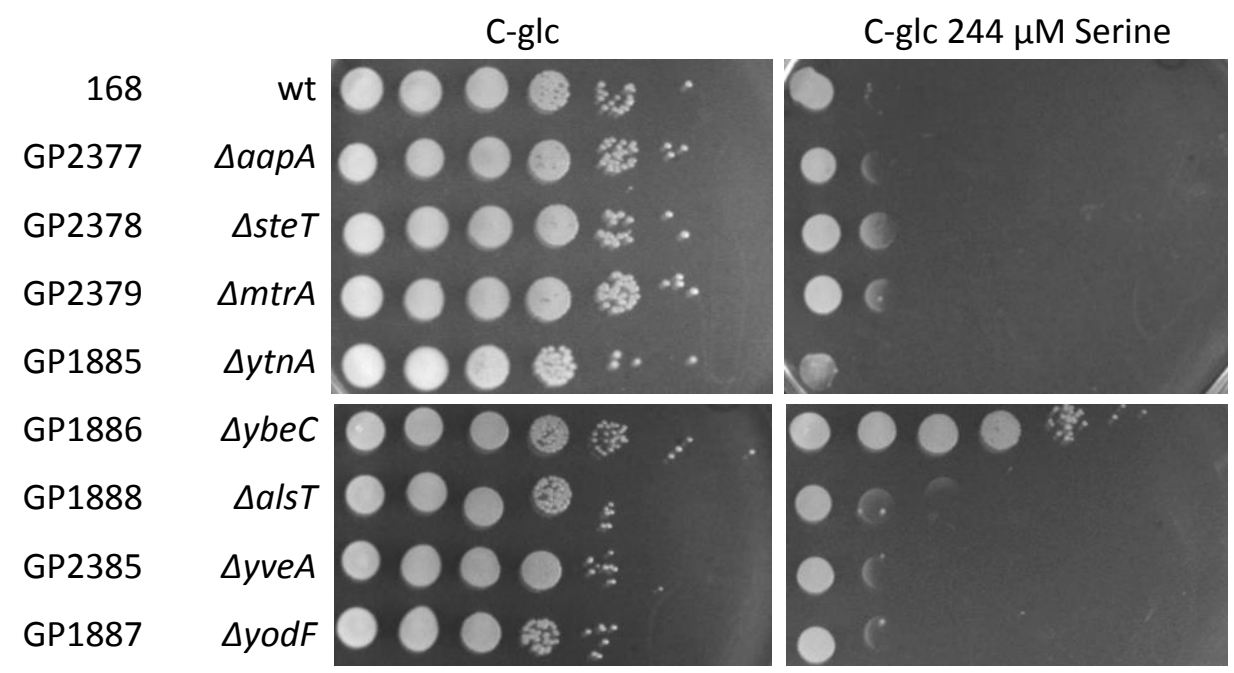

Figure 26: The drop dilution assay of the chosen potential transporters. A sample of $O D_{600}$ of 1 was prepared for each strain. The cells were diluted $10^{-1}$ in several steps and plated on C-glc-minimal plates with and without $244 \mu \mathrm{M}$ of one additional amino acid. The plates were grown for two days at $37^{\circ} \mathrm{C}$.

In the screening approach, performed in this work, the deletion strains were tested for their growth on C-glc minimal media with each one amino acid added. The aim was to observe differences in 
growth of mutant strains in comparison to the wild type. Interestingly, it could be observed, that the wild type strain 168 and all other mutants except for the ybeC deletion mutant showed a growth defect on C-glc plates with $244 \mu \mathrm{M}$ serine (Figure 26).

Serine alone in minimal plates was shown to be toxic for the cells, although the reason for the toxicity is still unknown (Lachowicz et al., 1996). However, the ybeC deletion mutant of seems to be resistant to serine. In theory, a deletion of a serine importer would lead to a decrease of serine import and therefore a minimization of the toxic effect. The next step was to test if the $\Delta y b e C$ mutant is also resistant on higher serine concentrations (Figure 27).

\begin{tabular}{|c|c|c|c|c|}
\hline & & C-glc $244 \mu \mathrm{M}$ Serine & C-glc $1 \mathrm{mM}$ Serine & C-glc 17 mM Serine \\
\hline 168 & wt & 8. & & \\
\hline
\end{tabular}

Figure 27: The growth of the $\Delta y b e C$ mutant in comparison to the wild type. The cells of the strains GP1886 and the wild type were diluted in several steps and plates on C-glc medium with $244 \mu \mathrm{M}, 1 \mathrm{mM}$ and $17 \mathrm{M}$ serine. The plates were grown for two days at $37^{\circ} \mathrm{C}$.

The $\Delta y b e C$ mutant is able to grow on an even higher serine concentration of $1 \mathrm{mM}$. However, if the concentration is increased further to even $17 \mathrm{mM}$, the strain is not able to grow anymore. In comparison, the wild type strain could not grow on $1 \mathrm{mM}$ serine and only weakly on $244 \mu \mathrm{M}$.

Furthermore, the emergence of suppressor mutants can be observed for the wild type strain 168 and the strain GP2392 ( $\Delta$ serA) and many of these were isolated and characterized. The suppressor wt 1 (GP2324) was isolated on C-glc plates with 1 mM serine and analysed by WGS. The strain harbours a single basepair deletion in the gene ybeC (bp 340), which leads to a frameshift and a shorter protein. This supports the theory, that the $\mathrm{YbeC}$ protein is responsible for the serine import. Several other isolated suppressor mutants were tested via PCR and sequencing for a mutation in ybec. Furthermore, another suppressor of the wild type strain, isolated on $1 \mathrm{mM}$ serine (GP2325), and two suppressors of the strain $\triangle$ serA (GP2392), isolated on C-glc plates with $244 \mu \mathrm{M}$ (GP3049 and GP3050), showed different mutations in the ybeC gene (Table 10). All four ybeC suppressor mutants show a better growth on C-glc plates with serine in comparison to their parental strains (Figure 28).

The $\Delta$ serA strain needs to take up serine from the medium, since it cannot synthesis it. However, the growth is very weak and comparable with the growth of the wild type strain. The suppressors that were isolated show a better growth on the minimal medium. Although the suppressor $\Delta$ serA 1 grows better on $244 \mu \mathrm{M}$ serine, the formation of new suppressors on $1 \mathrm{mM}$ serine can be observed. 


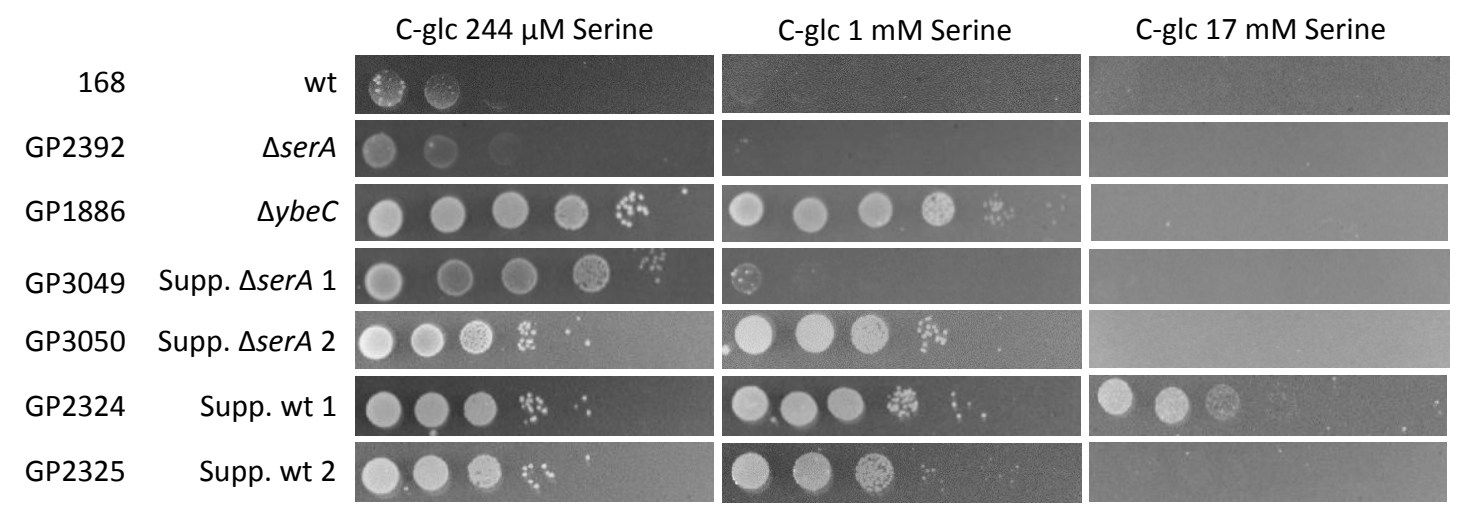

Figure 28: The drop dilution experiment of isolated suppressors in comparison to their parental strains and the $\Delta y b e C$ deletion sstrain. The suppressors of the wild type strain 168, GP2324 and GP2325 were isolated on C-glc plates with $1 \mathrm{mM}$ serine and the $\Delta$ serA suppressors GP3049 and GP3050 were isolated on C-glc paltes with $244 \mu \mathrm{M}$ serine. Precultures were prepared in C-glc medium and the plates were grown for two days at $37^{\circ} \mathrm{C}$.

The acquired mutation in this suppressor $\triangle$ serA 1 seems to be not as beneficial as the mutation in suppressor $\Delta s e r A 2$. This strain is able to grow on $1 \mathrm{mM}$ serine comparable to the $\Delta y b e C$ mutant. Interestingly, the suppressor wt 1 is even able to grow on $17 \mathrm{mM}$ serine.

Table 10: The suppressor mutations found in ybeC, isolated on C-glc serine plates.

\begin{tabular}{|c|c|c|}
\hline Strain & Suppressor mutant & Mutation in YbeC \\
\hline GP3049 & Suppr. $\Delta$ serA 1 & E522* \\
\hline GP3050 & Suppr. $\Delta$ serA 2 & $\begin{array}{l}\text { In frame deletion of } 708 \text { bp after bp } 306 \\
\text { (236 aa missing after aa 102) }\end{array}$ \\
\hline GP2324 & Suppr. wt 1 & $\begin{array}{l}\text { bp } 340 \text { is deleted, leading to a frameshift } \\
\text { and a shorter protein }\end{array}$ \\
\hline GP2325 & Suppr. wt 2 & $\begin{array}{l}\text { bp } 974 \text { is deleted, leading to a frameshift } \\
\text { and a shorter protein }\end{array}$ \\
\hline
\end{tabular}

Different mutations can be observed in the suppressor mutants. The mutation in the strain GP3049 is interesting, since it leads to a stop codon $\mathrm{n}$ the $\mathrm{C}$-terminus of the $\mathrm{YbeC}$ protein (compare Figure 29). This missing 18 amino acids seemed to lead to an impaired function of the protein, which indicates a special function of the C-terminal end of the protein. However, this suppressor mutant grows not as good as the deletion mutant of $y b e c$, which means, that the mutation of the Cterminus probably does not lead to a completely non-functional protein. The suppressor $\triangle s e r A 2$ in contrast, has a mutation of $708 \mathrm{bp}$, after bp 306, which leads to the precise deletion of 236 amino acids in the middle of the YbeC protein. The suppressor wt 2 (GP2325) harbours, similar to the strain GP2324, a single basepair deletion which leads to a frameshift and a shorter protein. Since the 
suppressor mutants GP3049, GP3050 and GP2325 were analysed by PCR and sequencing, further unidentified mutations might lead to the observed growth advantage in comparison to the parental strains.

Conclusively, the toxic effect of serine alone in the minimal plates can be compensated by the mutation of $y b e c$. This leads also to the assumption that $\mathrm{YbeC}$ is a serine importer and the suppressors are protected by the toxic effect of serine since the strains import less serine into the cell. Some of the isolated and analysed suppressor mutants showed no mutation in ybeC.

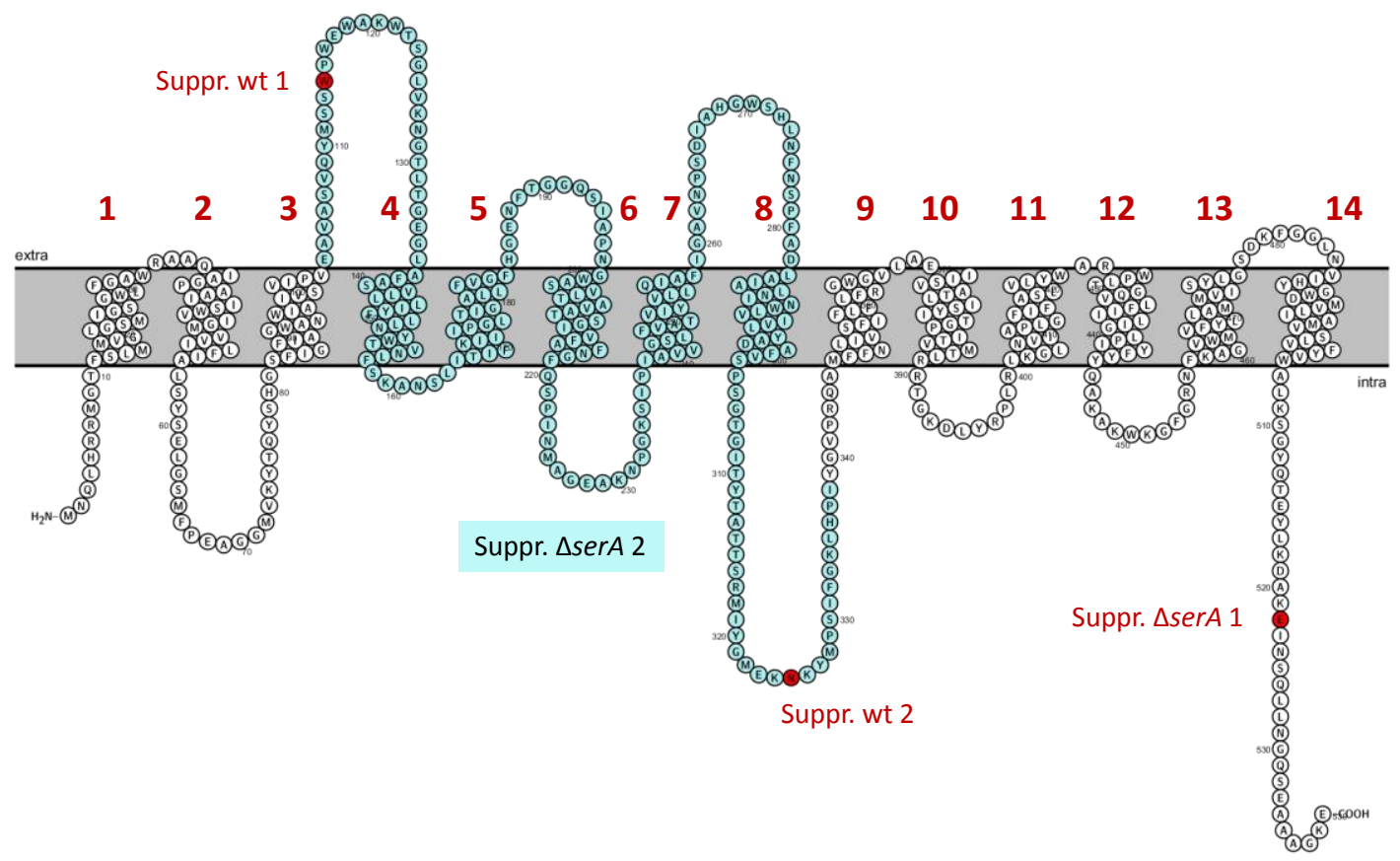

Figure 29: The observed suppressor mutations of the membrane protein YbeC. The amino acids marked in red are mutated in the corresponding suppressor mutants. The amino acids marked in blue are deleted in the suppressor $\Delta$ serA 2. The YbeC protein is shown according to UniProt and Protter (http://wlab.ethz.ch/protter) (Omasits et al., 2014).

Since the $\Delta y b e C$ mutation seems to decrease the uptake of serine, the emerging of suppressor mutants in the strain $\triangle$ serA (GP2392) is interesting. The gene serA codes for the phosphoglycerate dehydrogenase and the enzyme catalyses the first step in the biosynthesis of serine (Chi et al., 2013). The deletion mutant $\Delta s e r A$ is auxotrophic for serine and it is dependent on the import of serine. The strain GP2392 is therefore not able to grow on C-glc plates without serine. The ybeC suppressor mutation in the strain $\Delta$ serA indicates, that $\mathrm{YbeC}$ is not the only serine importer. To test this hypothesis the deletion mutation of ybeC was introduced into the strain $\triangle \operatorname{ser} A$ and the resulting strain GP2941 was tested for the growth on C-glc plates with serine (Figure 30). Similar to the serA deletion strain, the strain GP2941 is not able to grow on C-glc medium without serine. 
The growth of the double mutant $\Delta y b e C \Delta s e r A$ on C-glc medium with serine is comparable to the one of the $\triangle y b e C$ mutant. This leads to the assumption, that $\mathrm{YbeC}$ cannot be the only serine importer in B. subtilis, since the serine auxotrophic mutant with the ybeC deletion is still viable.

\begin{tabular}{|c|c|c|c|c|}
\hline & & C-glc & C-glc $244 \mu \mathrm{M}$ Serine & C-glc 1 mM Serine \\
\hline GP2392 & $\Delta s e r A$ & & 00 & \\
\hline GP1886 & $\Delta y b e C$ & $\therefore$ & O 0 종 $:$ & 00 is \\
\hline GP2941 & $\Delta y b e c \Delta s e r A$ & & $80.3 \therefore$ & 00 \\
\hline
\end{tabular}

Figure 30: The combination of the ybec deletion with the deletion of serA. The double deletion strain was compared to the single deletion strains of serA and $y b e C$. A serial dilution assay was performed for each strain and the cells were plated on C-glc medium with and without serine. The plates were grown for two days at $37^{\circ} \mathrm{C}$.

Furthermore, the $\Delta y b e C$ mutant shows better growth in the presence of a toxic derivative of serine, DL-serine hydroxamate, in comparison to the wild type (chapter 6.5. supplementary information). This also indicates, that the deletion strain of ybec takes up less serine or in this case toxic derivative, than the wild type.

The next step was to test if the expression of $y b e C$ is influenced by the presence of serine. Therefore, the $y b e C$ promoter fuzed to a lacz gene by cloning the promoter into the plasmid pAC5. The wild type strain 168 was transformed with the resulting plasmid pGP2287, which integrates into the amyE locus of the chromosome (Martin-Verstraete et al., 1992). The activity of this translational fusion of the $y b e c$ promoter to the lacz gene was measured with a $\beta$-galactosidase activity. The strain was grown in different media to an $\mathrm{OD}_{600}$ of 0.5 and the $\beta$-galactosidase activity was measured as described in chapter 2.2.7. (Table 11).

Table 11: The $\beta$-galactosidase activity assay of the ybeC promoter fused to lacz.

\begin{tabular}{|c|c|c|c|c|}
\hline Medium & C-glc & $\begin{array}{l}\text { C-glc } 244 \mu \mathrm{M} \\
\text { Serine }\end{array}$ & LB-glc & $\begin{array}{l}\text { C-glc } \\
+244 \mu \mathrm{M} \text { serine } \\
+500 \mu \mathrm{M} \\
\text { glutamate }\end{array}$ \\
\hline $\begin{array}{l}\beta \text {-galactosidase } \\
\text { activity [Miller units] }\end{array}$ & $144.8 \pm 2.11$ & $132.4 \pm 8.21$ & $135.2 \pm 9.67$ & $142.2 \pm 2.85$ \\
\hline
\end{tabular}

The activity of the promoter is very similar in all media. The addition of serine or serine with glutamate does not seem to influence the expression of $y b e C$ in comparison to the measurements in C-glc minimal medium. 
Interestingly, serine is also toxic to E. coli in high amounts (Hama et al., 1990). To study if YbeC can also mediate the serine import in E.coli, a copy of the ybeC gene from B. subtilis was cloned into the vector pWH844 and transformed into the E. coli strain JM109 (Krüger, unpublished data). The growth of this strain was compared with a strain harbouring the empty vector (EV) on plates with increasing serine concentrations (Figure 31).

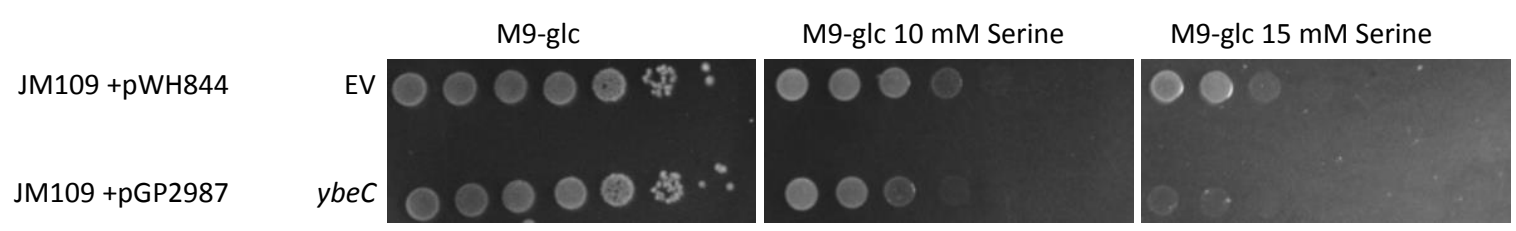

Figure 31: The growth of the E. coli strain JM109 with the ybeC plasmid pGP2987. Precultures were prepared in M9-glc medium and a serial dilution assay was performed and the cells were plated on M9-glc plates with or without serine. The plates were incubated for two days at $37^{\circ} \mathrm{C}$.

It can be observed, that the strain with the $y b e C$ plasmid (pGP2987) shows a growth disadvantage on M9 minimal plates with serine. The M9-glc medium was modified by removing all other amino acids to ensure that the toxic effect of serine can be seen (chapter 2.1.2.). It has to be mentioned, that the plasmid pWH844 is used to induce the expression of proteins by the addition of IPTG (Schirmer et al., 1997). However, there is no IPTG added to the M9-glc plates, but the basal expression from the plasmid seems to be sufficient to increase the sensitivity against serine. This experiment proves, that the $\mathrm{YbeC}$ protein can also act as a serine importer in E. coli. However, the suppressor analyses that was performed before, showed that an YbeC protein without the Cterminus is not completely functional. The plasmid pGP2988 harbours the modified ybec gene in the vector pWH844 (Krüger, unpublished data). The strain JM109 was transformed with the plasmid and also tested on M9-glc minimal plates with different concentrations of serine (Figure 32).

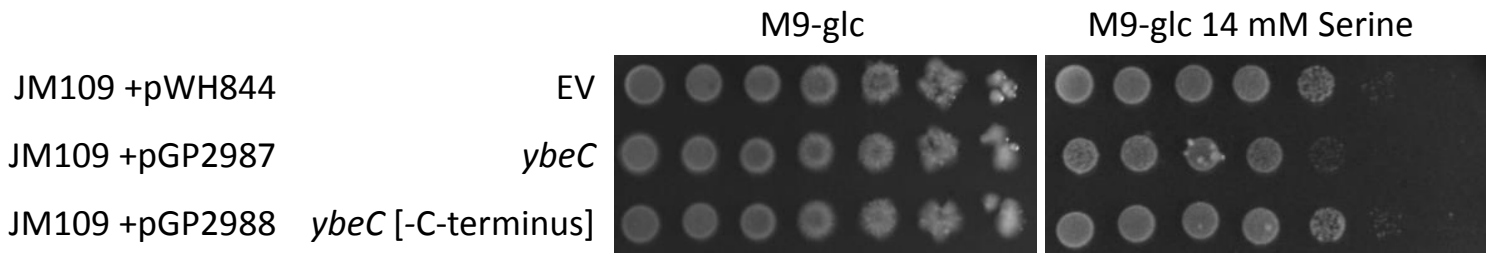

Figure 32: The growth of $E$. coli strains with different plasmids on M9-glc medium. The empty vector control was compared with a strain harbouring the $y b e C$ gene from $B$. subtilis and a strain with the $y b e C$ gene, lacking the C-terminus of the encoding protein. Precultures were prepared in M9-glc medium and the plates were incubated at $37^{\circ} \mathrm{C}$ for two days.

The $E$. coli strain with the $y b e C$ gene growths again worse than the EV control. However, the strain with the deletion of the C-terminus grows again as the EV control. These results indicate, that the YbeC protein is a serine importer and that it needs the C-terminus to form a functional protein. The 
deletion of the C-terminus might lead to a structural change of the protein, which leads to a nonfunctional protein. Furthermore, the C-terminal part of the $\mathrm{YbeC}$ protein could be important to sense a signal from the cell, which leads then to the activation of the import.

To ensure that the phenotype of the $\Delta y b e C$ deletion in $B$. subtilis is caused by the absence of the protein, a complementation assay was performed in which a new copy of ybeC on the plasmid pGP2980, was integrated into the $y k d E$ locus of the $\Delta y b e C$ strain (GP2948). The plasmid pGP2980 is based on the plasmid pGP886 (Gerwig et al., 2014; Krüger, unpublished data). The growth of the strain GP2948 on C-glc plates with xylose were compared to the strain GP2947 with the integrated empty vector (Figure 33).

\begin{tabular}{|c|c|c|c|}
\hline & C-glc $1 \%$ Xylose & $\begin{array}{l}\text { C-glc } 1 \% \text { Xylose } \\
244 \mu \mathrm{M} \text { Serine }\end{array}$ & $\begin{array}{l}\text { C-glc } 1 \% \text { Xylose } \\
1 \mathrm{mM} \text { Serine }\end{array}$ \\
\hline 168 & wt $00000 \div$ & $00 \div$ & 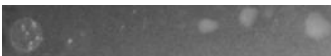 \\
\hline 1886 & $\Delta y b e c \bigcirc$ & 00000 & 00008 \\
\hline P2947 & $\Delta y b e c+\mathrm{EV} O \mathrm{OO}$ & 00000 : & $00008=$ \\
\hline 2948 & $\Delta y b e c P_{x y l}-y b e c 00$ & $0 O C$ & 29 \\
\hline
\end{tabular}

Figure 33: The complementation of the $\Delta y b e C$ mutant with a xylose inducible copy of $y b e C$. The strains were grown in C-glc medium and the serial drop dilution assay was performed on C-glc plates without and $244 \mu \mathrm{M}$ or $1 \mathrm{mM}$ serine. The $\mathrm{C}$-glc plates are incubated at $37^{\circ} \mathrm{C}$ for two days.

The expression of the new copy of the $y b e C$ gene can be induced by the addition of xylose. An empty vector integration into the $\Delta y b e C$ strain shows no difference on C-glc plates with serine, even in the presence of $1 \%$ xylose. However, if $y b e C$ is expressed in the $\triangle y b e C$ strain by the addition of xylose, the growth is similar to the growth of the wild type strain. This confirms the previous results that $y b e C$ is the reason for the resistance against serine and the effect is independent from the localisation on the chromosome.

To conclude, YbeC is a serine importer from $B$. subtilis. The deletion mutant is more resistant to serine in minimal medium than the wild type. Suppressor mutants of the wild type strain 168 and the $\Delta$ serA mutant emerge on C-glc plates with serine. Some of these mutations affect the ybeC gene. Furthermore, it is possible to introduce $y b e C$ into $E$. coli, which makes the strain more sensitive towards serine. However, $\mathrm{YbeC}$ is not the only serine importer in B. subtilis, since the combination with a serine auxotrophic mutant $\Delta s e r A$ is possible.

\subsubsection{Additional serine importers BcaP and YbxG}

The experiments in chapter 3.3.1. strongly suggest, that there are more serine importer than only YbeC. The combination of another serine importer deletion with $\Delta y b e C$ would lead to a strain that shows either an even better growth on C-glc serine medium when there are still more serine transporters. The combined strain could also show a worse growth than the $\Delta y b e C$ mutant, if all 
serine importers are deleted and the synthesis of serine is not enough to sustain the growth. The protein SteT was already mentioned as a potential serine / threonine exchanger and the deletion mutant $\Delta s t e T$ was combined with the $\triangle y b e C$ mutant. The growth of the single and double mutant was tested on C-glc serine plates (Figure 34).

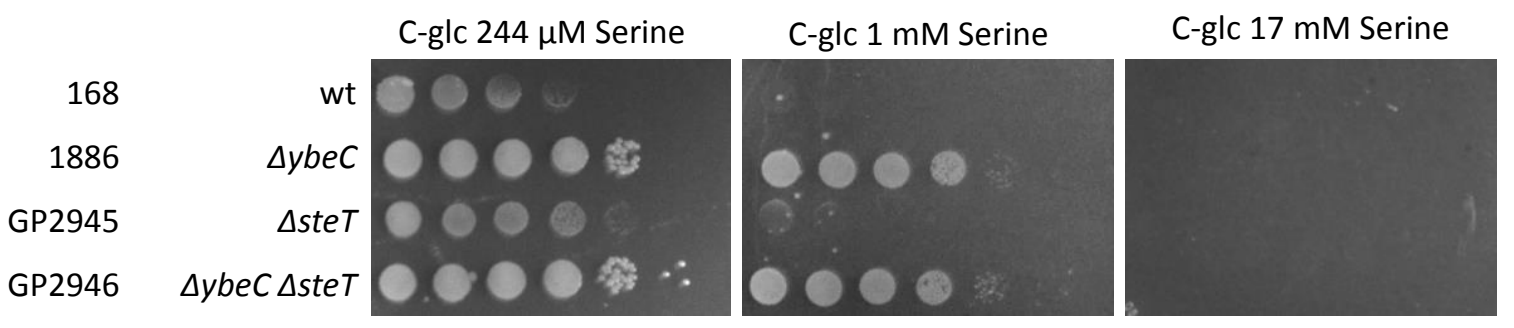

Figure 34: The gene steT was tested for its potential role in serine import on C-glc serine medium. The growth of the double deletion $\Delta y b e C \Delta s t e T$ was compared to the growth of the single deletion mutants at $37^{\circ} \mathrm{C}$. The plates were incubated for two days.

The additional deletion of ste $T$ in the $\triangle y b e C$ mutant shows no difference to the growth of the single deletion $\Delta y b e C$. Furthermore, the growth of the $\Delta s t e T$ single deletion has also no growth advantage on C-glc minimal medium with serine. These data indicate that the protein SteT has no function in the uptake of serine under the tested conditions.

Serine is often imported by the same protein that also imports threonine, e.g. in E. coli (Hama et al., 1987). Therefore, the known threonine transporters of $B$. subtilis were analysed for their potential role in the serine import. The transporter BcaP was shown to import isoleucine, valine and threonine. The import of isoleucine was shown to decrease if threonine, serine, valine, leucine, cysteine and asparagine was present in the medium (Belitsky, 2015). This leads to the assumption, that BcaP might also import serine into the cell. Furthermore, the protein YbxG was described as a potential threonine transporter since the gene was mutated in a suppressor that was found on the toxic threonine derivative 4-hydroxy-L-threonine (Commichau et al., 2015). The genes $b c a P$ and $y b x G$ were analysed as single deletion mutants and in combination with the ybeC deletion for their growth on C-glc serine (Figure 35).

The single deletion mutants of $b c a P$ and $y b x G$ grow slightly better than the wild type on Cglc medium with $244 \mu \mathrm{M}$ serine. If the strains are combined with the $\Delta y b e c$ deletion, the growth seems to be better in comparison to the $\triangle y b e C$ single deletion. Interestingly, the strain $\Delta y b e C \Delta b c a P$ $\Delta y b x G$ grows even on $17 \mathrm{mM}$ serine, which means the strain is highly resistant to the toxic effect of serine. This strongly indicates, that the $\triangle y b e C \triangle b c a P \Delta y b x G$ strain imports less serine than the $\triangle y b e C$ single deletion. Therefore, $\mathrm{YbxG}$ and BcaP are most likely playing a role in serine uptake. 


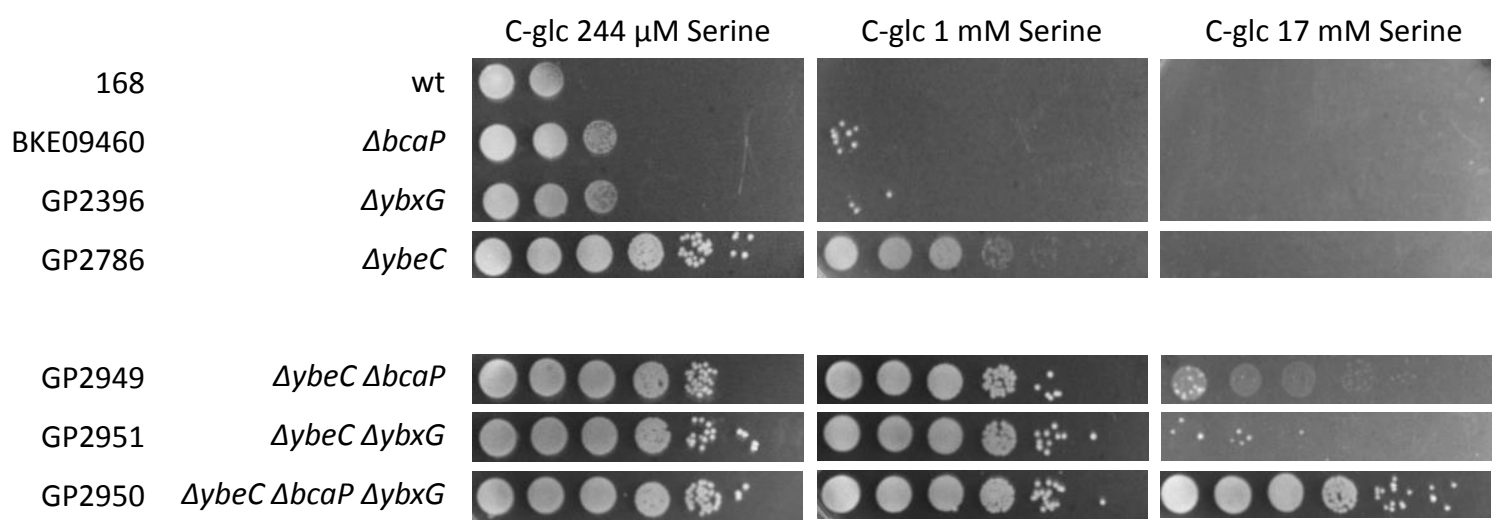

Figure 35: The drop dilution assay to test the resistance of the $\Delta b c a P$ and $\Delta y b x G$ to serine. The single mutants were combined with the deletion of the known transporter ybeC. Precultures were grown in C-glc medium. A serial drop dilution was performed and the plates were incubated for two days at $37^{\circ} \mathrm{C}$.

Since only three serine concentrations were tested, the strains could possibly show more growth differences. Therefore, a wide range of serine concentrations were tested, and the highest serine concentration was noted on which the strains could still grow. This was analysed one and two days after inoculation on C-glc plates (Figure 36).

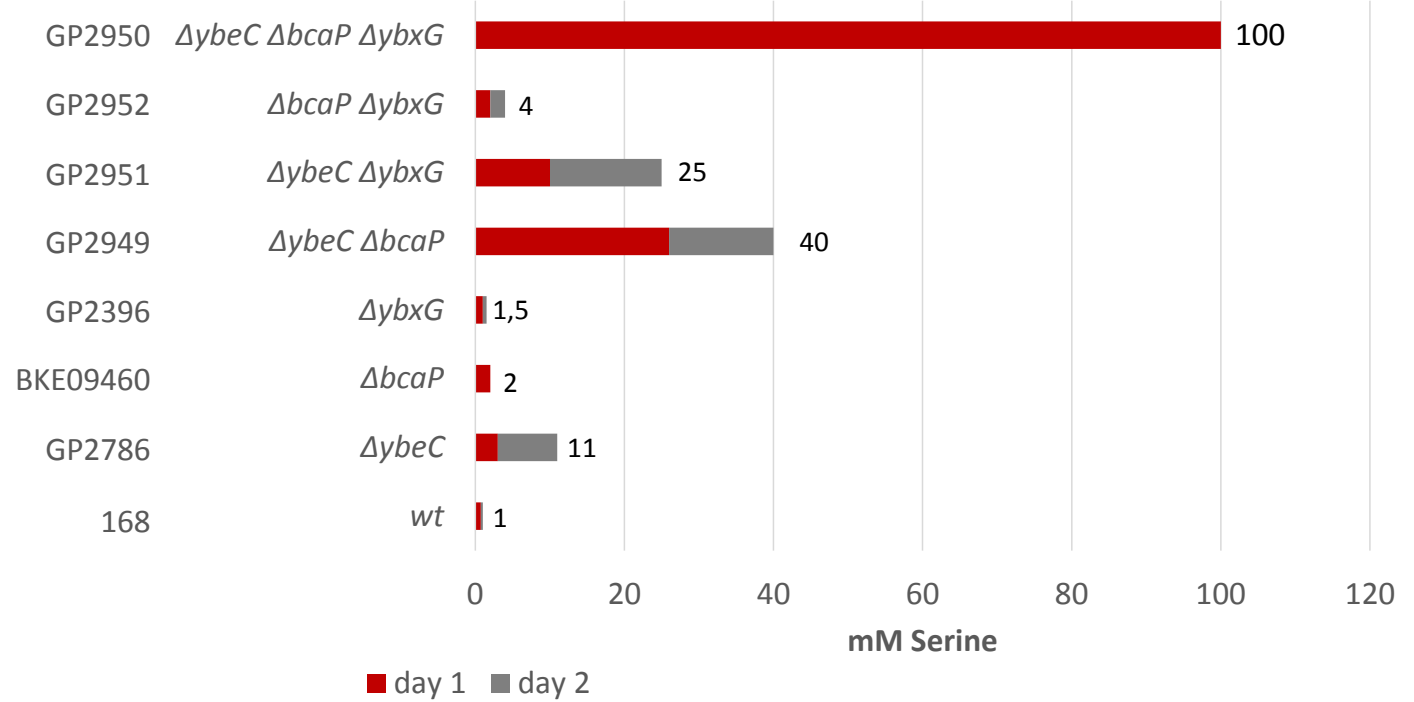

Figure 36: The different deletion strains of potential serine importers are tested for their growth of serine. The growth was observed one and two days after inoculation and the numbers indicate the highest concentration of serine that the mutant was able to grow on, on the second day.

The wild type was able to grow weakly on $1 \mathrm{mM}$ serine after day two. In contrast, the $\Delta y b e C$ strain grows on up to $11 \mathrm{mM}$ serine. The deletion mutants of the threonine and potential serine transporters $b c a P$ and $y b x G$, showed a higher resistance to serine than the wild type, but not as good as the $\triangle y b e C$ strain. So, the serine import by BcaP and YbxG seems to be less than the import of YbeC under these conditions. The double deletion mutant $\triangle b c a P \Delta y b x G$ can tolerate up to $4 \mathrm{mM}$ serine in the medium, which also fits to the assumption that the two proteins are involved in serine 
uptake. The combination of the $\triangle y b e C$ strain with either the $\triangle b c a P$ or the $\triangle y b x G$ deletion leads to strains, that are capable to grow on higher serine concentrations than the $\Delta y b e C$ single deletion. However, the deletion of $b c a P$ has a greater impact than the deletion of $y b x G$, which could also hint to differences in the uptake efficiency of the two proteins. Therefore, BcaP seems to import more serine into the cell, than $\mathrm{YbxG}$. Interestingly, the combined deletion of all three genes $\Delta y b e C \Delta b c a P$ $\Delta y b x G$ leads to a strain, that is highly resistant to serine. It can tolerate up to $100 \mathrm{mM}$ serine in the $\mathrm{C}$-glc plates. These growth differences of the single and combined strains could also be shown in growth curves in liquid C-glc medium (Figure 37).

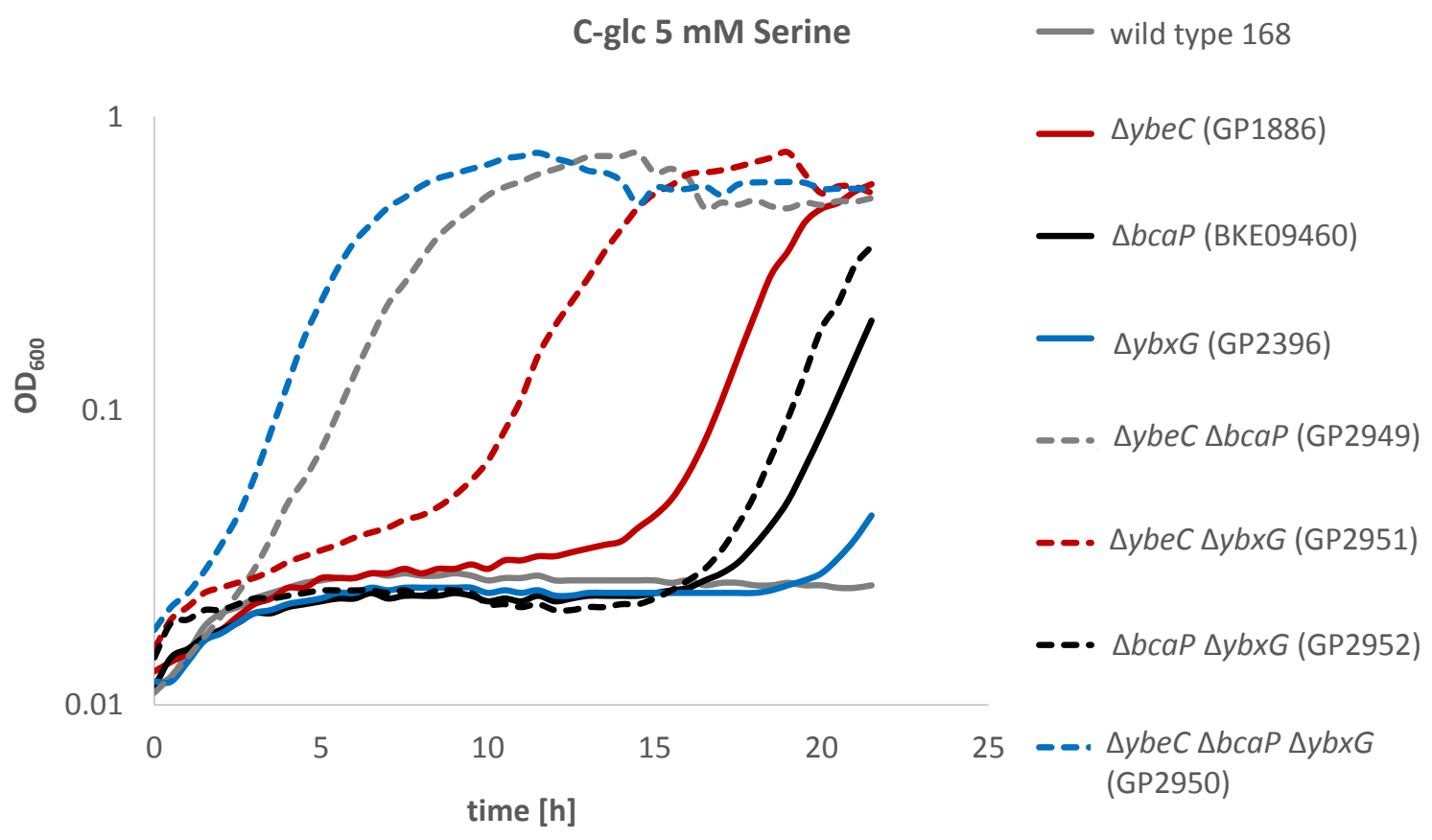

Figure 37: The growth curves of the single and combined deletion mutants of the serine importers in C-glc medium with $5 \mathrm{mM}$ serine. Precultures of all strains in $\mathrm{C}$-glc were used to inoculate the $\mathrm{C}$-glc medium with 5 $\mathrm{mM}$ serine. The growth was analysed at $37^{\circ} \mathrm{C}$.

The 168 wild type cannot grow with $5 \mathrm{mM}$ serine in C-glc liquid medium at all. The single deletion strains show the same order of resistance against serine, like it was observed before. The $\Delta y b e C$ strain grows the best, followed by the $\triangle b c a P$ strain and finally the $\triangle y b x G$ strain. Combining the bcaP and $y b x G$ deletion leads to a strain that grows better than the single mutants, but not as good as the $\triangle y b e C$ strain. The combination of $\triangle y b e C$ with the deletion of bcaP is cleary more beneficial for the growth in these serine concentrations than the combination with the $y b x G$ deletion. However, it can be observed that the triple deletion strain $\triangle y b e c \triangle b c a P \Delta y b x G$, grows without a growth disadvantage in these media. These results confirm the role of all three proteins in the import of serine. 
To analyse if the transporters could also import serine into $E$. coli cells, the $B$. subtilis genes $b c a P$ and $y b x G$ were also cloned into the pWH844 vector and transferred into the E. coli strain JM109. The resulting strains were compared with the EV and the strains with the complete ybeC gene and the $y b e C$ gene without the C-terminal part of the protein (Figure 38).

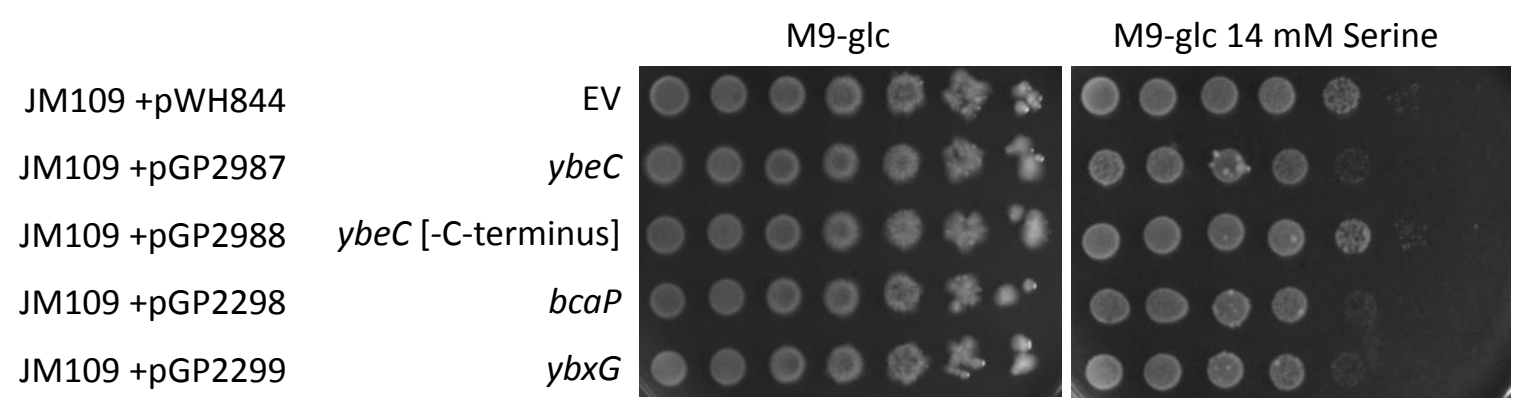

Figure 38: The drop dilution assay of $E$. coli strain harbouring the plasmids encoding for the $B$. subtilis serine importers. The genes encoding for serine transporters $y b e C, b c a P$ and $y b x G$ were cloned into a plasmid and transferred into $E$. coli JM109 cells. Additionally, the ybeC gene without the C-terminal part of the protein was tested. The plates were incubated at $37^{\circ} \mathrm{C}$ for two days.

The $E$. coli strains with the plasmid integrated gene ybeC shows again a growth disadvantage on $\mathrm{M9}$-glc plates with serine. In contrast, the $y b e \mathrm{C}$ mutant without the $\mathrm{C}$-terminal part of the protein growth like the strain with the EV, since the protein is not functional. Interestingly, the bcaP and $y b x G$ strains also show a similar growth disadvantage than the strain with $y b e c$. The reason for that might be the expression in a different organism. The regulatory mechanism that control the expression and activity of the proteins might be only existing in B. subtilis but not in E. coli. Furthermore, the E. coli strain JM109 still harbours all serine importers of E. coli. A very severe growth disadvantage could be adjusted by regulating the native $E$. coli serine importers, like SstT (Ogawa et al., 1997). Additionally, the expression of the plasmid coded genes is not induced by IPTG in these experiments. The basal, leaky transcription is enough to produce a few proteins that are responsible for this effect. However, these results show that the E. coli strains with the plasmid based $y b e C, b c a P$ and $y b x G$ take up more serine, which is in certain concentrations also toxic for $E$. coli.

The very high resistance of the $B$. subtilis triple deletion strain $\Delta y b e C \Delta b c a P \Delta y b x G$ to serine could indicate that all serine importers of $B$. subtilis might be deleted in this strain. To test this hypothesis, the gene serA was deleted in the triple deletion strain. It was already mentioned, that serA is involved in the biosynthesis of serine and a deletion of it leads to a serine auxotrophic strain. However, the deletion of serA was possible in the $\Delta y b e c \Delta b c a P \Delta y b x G$ deletion strain and the cells were viable. The resulting strain was then tested for its growth on C-glc medium with and without serine (Figure 39). 


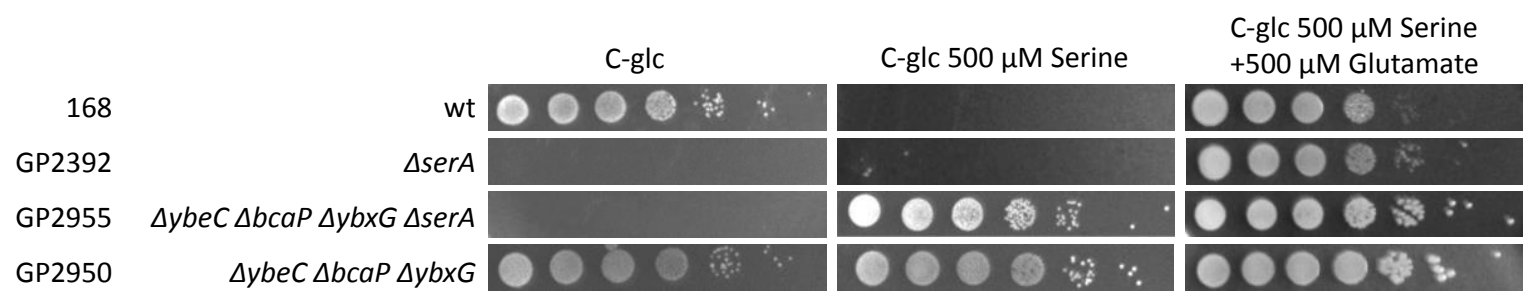

Figure 39: The growth of the strain GP2955 ( $\Delta y b e C \Delta b c a P \Delta y b x G \Delta s e r A$ ) compared to the $\Delta$ serA deletion mutant and the triple deletion strain GP2950. The growth was tested on C-glc medium, on C-glc medium with $500 \mu \mathrm{M}$ serine and on C-glc medium with $500 \mu \mathrm{M}$ serine and $500 \mu \mathrm{M}$ glutamate. The plates were incubated at $37^{\circ} \mathrm{C}$ for two days

The single deletion strain $\Delta s e r A$ is not able to grow on C-glc medium without additional serine, since it is serine auxotrophic. The combined mutant $\triangle y b e c \Delta b c a P \Delta y b x G \Delta s e r A$ shows also no growth on the C-glc medium, which means the gene serA was successfully deleted and the strain is indeed serine auxotroph. However, this strain is also still able to grow on C-glc plates with $500 \mu \mathrm{M}$ serine like the strain without the serA deletion. This means, that the import of serine is still possible, although three serine importers are deleted. So $\mathrm{YbeC}, \mathrm{BcaP}$ and $\mathrm{YbxG}$ are not the only membrane proteins that are able to take up serine from the medium. However, the amount of imported serine by the missing transporter is just enough that the cells do not suffer.

To conclude, the transporter BcaP is not only able to import valine, isoleucine and threonine into the cell, but also serine. The potential threonine transporter YbxG plays also a role in serine import, but the amount of serine transported into the cell by BcaP is higher. Nevertheless, YbeC seems to be the main importer of serine under the tested conditions, since a deletion of the $y b e c$ gene leads to the highest resistance against high serine concentrations.

\subsubsection{Threonine uptake function of YbeC, BcaP and YbxG}

The protein BcaP was described to transport threonine into the cell and also YbxG could have a threonine import function (Belitsky, 2011; Commichau et al., 2015). Therefore, the role of bcaP, $y b \times G$ and also ybeC in threonine import should be analysed in this chapter. YbeC could also import threonine, since threonine and serine are structurally similar, and the transport of both amino acids is often coupled. The growth of the single, double and triple deletion mutants of the transporters $y b e C, b c a P$ and $y b x G$ was tested in C-glc medium with $10 \mathrm{mM}$ threonine (Figure 40). 


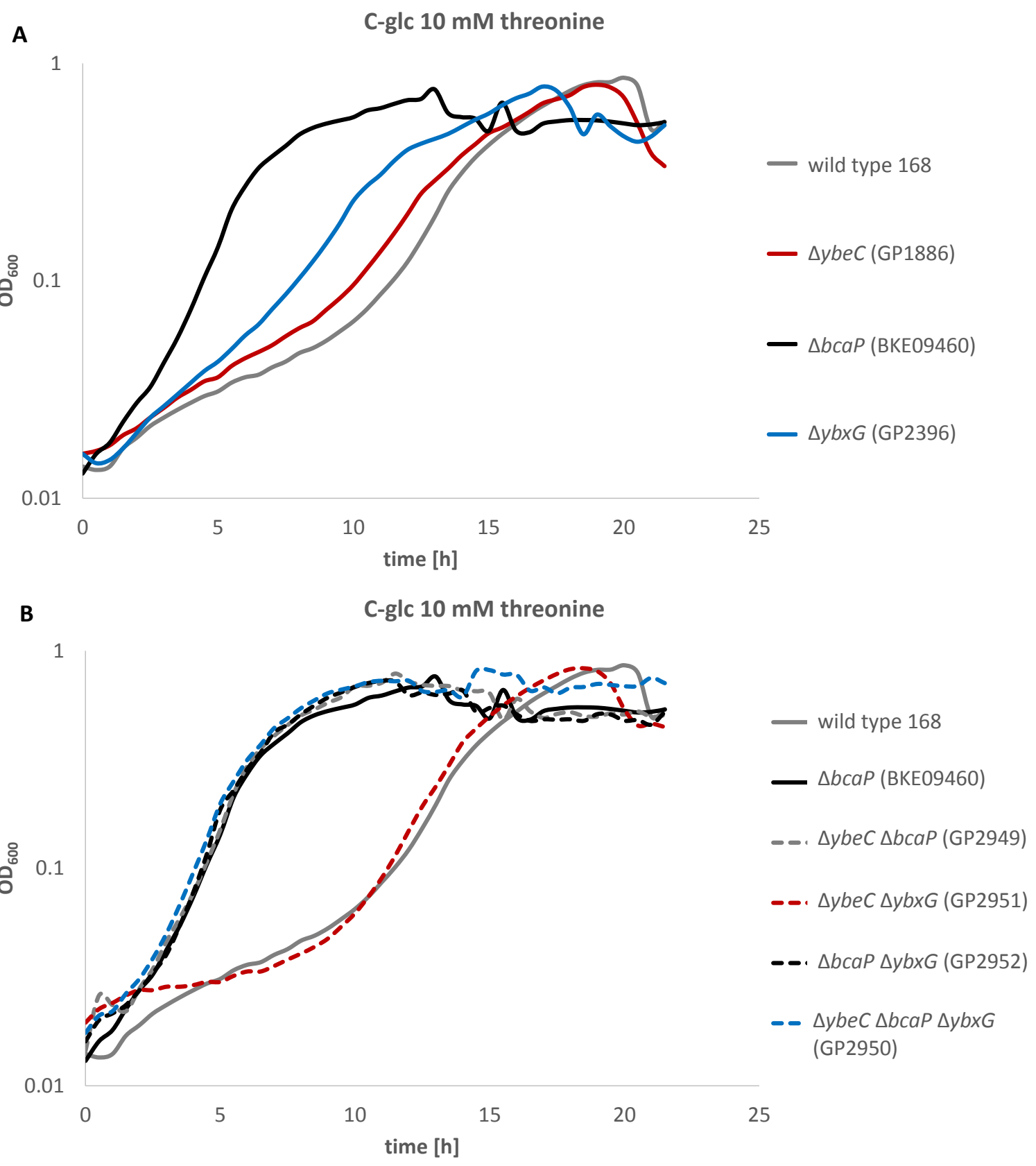

Figure 40: The growth of the potential threonine importer deletion strains in C-glc medium with $10 \mathrm{mM}$ threonine. Precultures were prepared in LB medium. A The single deletion mutants of $y b e C, y b x G$ and $b c a P$ are compared to the wild type strain 168. B The growth of the double and triple deletion strains of $y b e C, y b x G$ and $b c a P$ are compared to the growth of the wild type strain 168 and the bcaP deletion strain. The growth was analysed at $37^{\circ} \mathrm{C}$.

Interestingly, high concentrations of threonine are toxic for $B$. subtilis, since it inhibits growth and sporulation (Lamb and Bott, 1979b). The $\Delta b c a P$ deletion mutant has a clear growth advantage in comparison to the wild type strain 168 , which means that $\mathrm{BcaP}$ is responsible for the uptake of threonine. However, also the $\Delta y b x G$ mutant can transport threonine, since it shows a better growth under these conditions. The ybec deletion mutant in contrast grows just a little bit better than the wild type. This probably means, that a small amount of threonine can be imported to the cell by 
$\mathrm{YbeC}$, but since the effect is not as high as in the case of serine, the import is not as high as the import of serine. However, the double deletion strains that harbour a deletion in bcaP and also the triple deletion strain $\triangle y b e c \Delta b c a P \Delta y b x G$ grow, like the $\Delta b c a P$ single deletion, very good under these conditions. This indicates that BcaP is the main low-affinity transporter for threonine. In contrast, the strain $\Delta y b e c \Delta y b x G$ has almost no growth advantage in comparison to the wild type, which indicates a weak threonine import function of the both proteins. It needs to be noted, that the growth of the $\Delta y b e C \Delta y b x G$ deletion mutant is even worse than the growth of the $\Delta y b x G$ single deletion strain, but the reason for this is unclear.

To analyse if all threonine transporters are deleted in the strain $\Delta y b e C \Delta b c a P \Delta y b x G$, the strain was combined with a deletion of $t h r C$. The gene $\operatorname{thr} C$ encodes for the threonine synthase, a protein of the threonine biosynthesis pathway. A $\Delta t h r C$ deletion mutant is auxotrophic for threonine. The created deletion mutant was viable and it was tested for its growth in C-glc medium with varying concentrations of threonine (Figure 41).

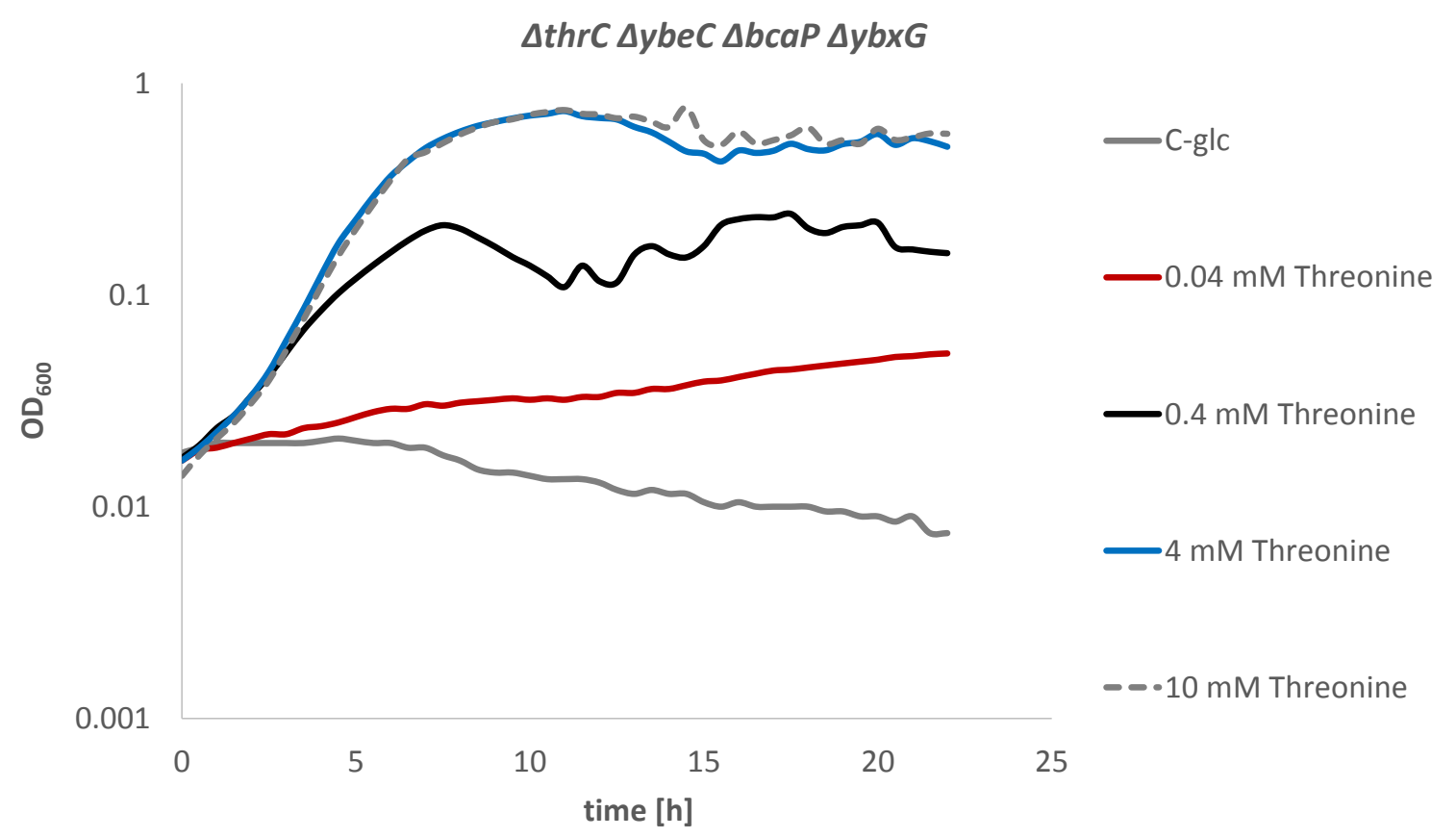

Figure 41: The growth of GP3037 ( $\Delta$ thrC $\Delta y b e C \Delta b c a P \Delta y b x G$ ) in C-glc medium with different concentrations of threonine. Precultures for this experiment were grown in LB medium. The growth was analysed in the plate reader at $37^{\circ} \mathrm{C}$.

The strain cannot grow in C-glc medium, which indicates, that thrC was successfully deleted and the strain is auxotrophic for threonine. A concentration of $0.04 \mathrm{mM}$ threonine is not enough to sustain a robust growth. This might be due to the reason that the strain needs a higher threonine concentration to efficiently take up the amino acid. If the threonine concentration is further increased to $0.4 \mathrm{mM}$, the strain grows better in comparison to the lower concentration, but not as 
good as with 4 or $10 \mathrm{mM}$ threonine. The $10 \mathrm{mM}$ threonine concentration was previously shown to be toxic for the cell, but the strain $\Delta t h r C \Delta y b e c \Delta b c a P \Delta y b x G$ is resistant to it.

Furthermore, the gene thrC was deleted in all single and double deletion strains of the potential threonine transporters $b c a P, y b x G, y b e C$. The resulting strains were also tested in C-glc medium with different concentrations of threonine and the results are shown in Figure 42.

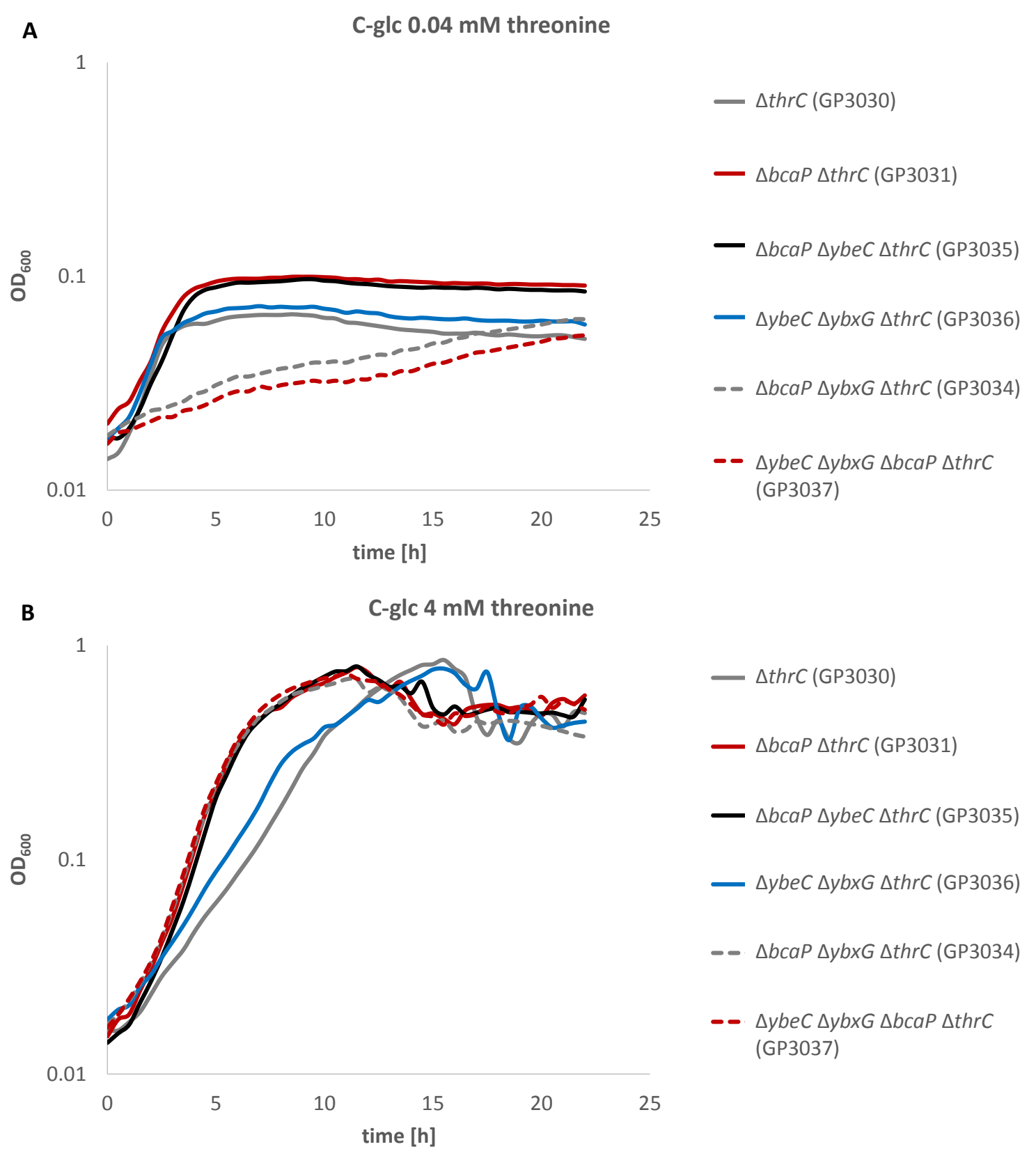

Figure 42: The growth of the potential threonine importer deletion strains in the background of $\Delta t h r C$. The strains were grown at $37^{\circ} \mathrm{C}$ in C-glc medium with $0.04 \mathrm{mM}$ threonine (A) and $4 \mathrm{mM}$ threonine (B).

Growth of the $\Delta t h r C$ strains in $0.04 \mathrm{mM}$ threonine is overall weak, and the $\Delta t h r C \Delta b c a P$ deletion strain grows the best in this medium. As already mentioned, the deletion of all three transporters and $t h r C$ leads to a reduced growth. Interestingly, this is also the case for the $\Delta \operatorname{thr} C \Delta b c a P \Delta y b x G$ 72 
deletion strain. The absence of the proteins BcaP and $\mathrm{YbxG}$ seems to have a more severe effect, than the absence of $\mathrm{YbeC}$ and $\mathrm{BcaP}$ or $\mathrm{YbxG}$. This indicates, that if $\mathrm{YbeC}$ has a role in the uptake of threonine, it might be a rather small one. In contrast, the major role of BcaP in the uptake of threonine can be observed in the growth curves at $4 \mathrm{mM}$ threonine. The only strain, in which the gene bcaP is not deleted ( $\Delta \operatorname{thr} \triangle \mathrm{ybeC} \Delta y b x G$ ) grows the worst. This might be caused by the threonine concentration of $4 \mathrm{mM}$, that is already harmful for the cell. The remaining transporter BcaP imports high amounts of threonine into the cell. Therefore, BcaP seems to be the main transporter for threonine.

Interestingly, threonine can counteract the toxic effect of serine to a certain limit, if the two amino acids are present in the medium (Figure 43).

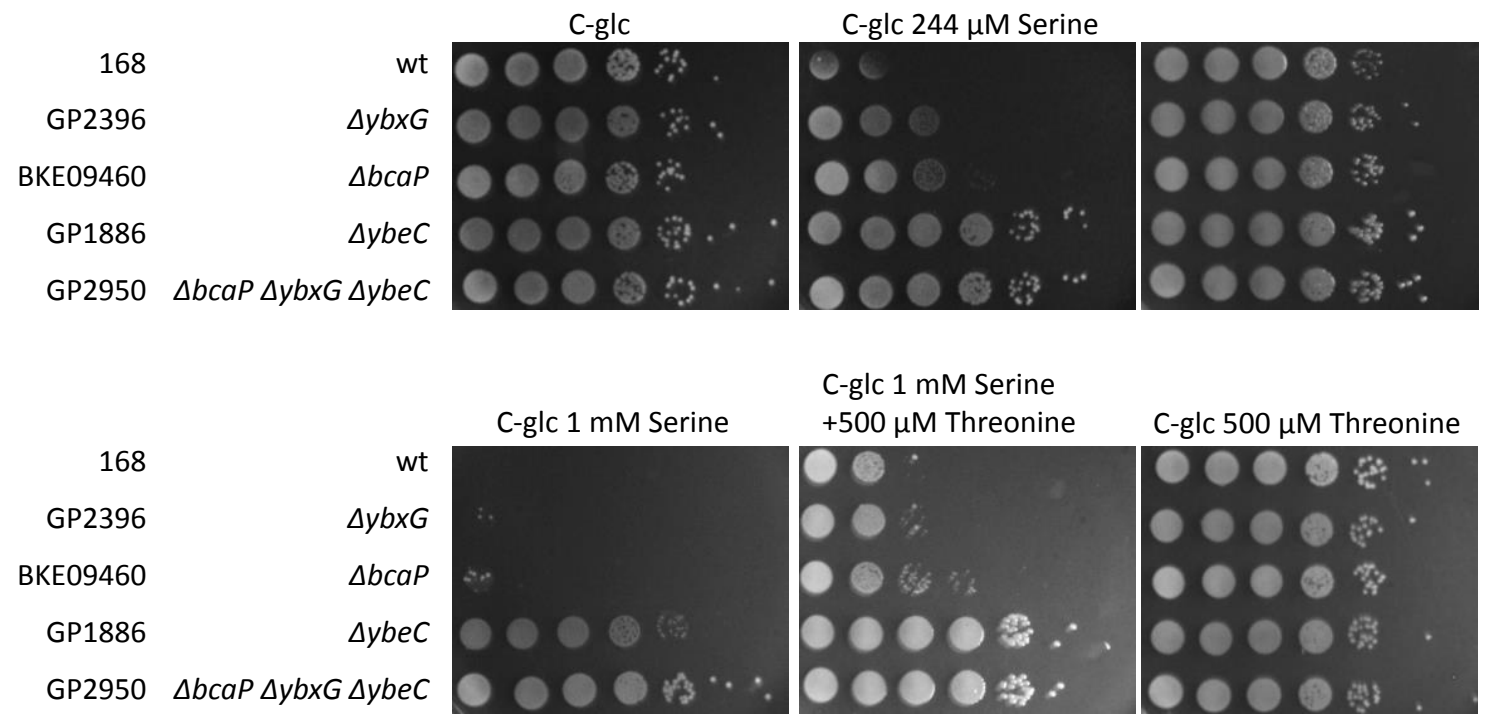

Figure 43: The drop dilution assay on different serine and threonine concentrations. The growth of the single deletion strain of ybec (GP1886), ybxG (GP2396) and bcaP (BKE09460) is compared to the triple deletion strain GP2950. The C-glc plates contain $244 \mu \mathrm{M}$ or $1 \mathrm{mM}$ serine with and without $500 \mu \mathrm{M}$ serine. The C-glc plates without additional amino acid serves as a control.

The wild type strain 168 , the $\Delta y b x G$ and the $\triangle b c a P$ mutants show a growth defect on C-glc plates with $244 \mu \mathrm{M}$ and $1 \mathrm{mM}$ serine. However, the addition of $500 \mu \mathrm{M}$ threonine to the medium with $244 \mu \mathrm{M}$ serine can compensate this observed growth defect. This is also possible for $1 \mathrm{mM}$ serine with $500 \mu \mathrm{M}$ threonine, but the growth disadvantage is again visible. Furthermore, $500 \mu \mathrm{M}$ threonine alone in C-glc plates is not toxic for the strains. This indicates, that serine and threonine are transported by the same importer into the cell. If only serine is present, the importer only transports serine, but if threonine is also present, the two amino acids are imported into the cell. Therefore, a lower amount of serine is imported into the cell and the toxic effect is less pronounced. Another possibility is that a higher threonine concentration in the cell can compensate directly the toxic effect of serine. This will be further analysed and discussed in chapter 3.4. 
To conclude this chapter, the transporter BcaP is the main threonine importer of B. subtilis. However, the protein YbxG is also able to transport threonine into the cell. The previously identified serine transporter YbeC could also have a function in threonine import, but the amount of threonine transported by the protein is just very small. However, these are not all of the threonine importers in B. subtilis, since the deletion strain $\Delta \operatorname{thr} \triangle \Delta y b e C \Delta b c a P \Delta y b x G$ is still viable, although it is auxotrophic for threonine.

\subsection{The toxic effect of serine}

High concentrations of serine are toxic for the growth of $B$. subtilis. Although, this observation is published in the literature, the mechanism itself and also the physiological role of it is unknown (Lachowicz et al., 1996; Koo et al., 2017). Therefore, the target of the serine toxicity in B. subtilis should be analysed further in this work.

It was already shown that a mutation in the $y b e C$ gene, encoding for a serine importer can cause resistance to serine (chapter 3.3.1.). This indicates that the toxic effect of serine acts on something in the cell. However, the suppressor mutations in ybec were not the only suppressor mutants that were isolated on high concentrations of serine (Figure 44).

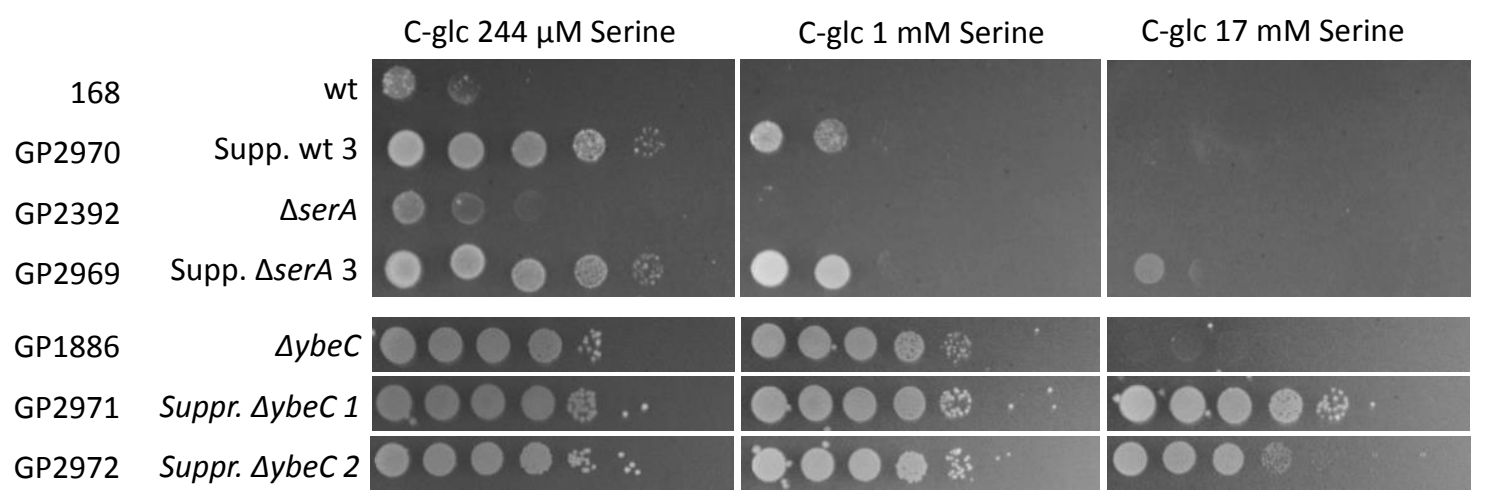

Figure 44: The drop dilution experiment of the isolated suppressor mutants in comparison to the parental strains. GP2970 was solated from the wild type strain 168, grown on C-glc with $244 \mu \mathrm{M}$ serine. Strain GP2969 derives from the $\triangle$ serA strain GP2392, grown on $244 \mu \mathrm{M}$ serine. The strains GP2971 and GP2972 were isolated from C-glc plates containing $10 \mathrm{mM}$ and $17 \mathrm{mM}$ serine and both derive from the $\triangle y b e C$ strain GP1886. A serial dilution was performed for all strains and the cells were plated on C-glc plates with $244 \mu \mathrm{M}, 1 \mathrm{mM}$ and 17 $\mathrm{mM}$ serine. The plates were incubated at $37^{\circ} \mathrm{C}$ for two days.

Suppressor mutants from the wild type strain 168 and the $\Delta$ serA strain (GP2392) were isolated, since they appear fast on C-glc plates with $244 \mu \mathrm{M}$ and $1 \mathrm{mM}$ serine. The suppressor wt 3 (GP2970) and the suppressor $\Delta$ serA 3 (GP2969) were isolated on $244 \mu \mathrm{M}$ serine and a PCR and sequencing analysis of each $y b e c$ gene showed no mutation. The $y b e c$ deletion strain cannot grow on high serine concentrations and the emerge of suppressor mutants can also be observed. The suppressor mutants GP2971 and GP2972 were isolated on C-glc plates with $10 \mathrm{mM}$ and $17 \mathrm{mM}$ serine. All of 
these isolated mutants grow better than their parental strains. To identify the suppressor mutation of each strain, WGS was applied to all suppressor strains (Table 12).

Table 12: The serine suppressor mutants and their identified mutations

\begin{tabular}{|l|l|l|l|}
\hline Strain & Parental strain & Isolated on & Mutation found in WGS \\
\hline GP2969 & GP2392 $(\Delta s e r A)$ & $244 \mu \mathrm{M}$ serine & $t h r R^{*}(\mathrm{bp} 90$ deleted $\rightarrow$ frameshift $)$ \\
\hline GP2970 & 168 & $244 \mu \mathrm{M}$ serine & $\begin{array}{l}\text { Duplication /amplification of a genomic } \\
\text { region, including } i / v A\end{array}$ \\
\hline GP2971 & GP1886 $(\Delta y b e C)$ & $10 \mathrm{mM}$ serine & sdaAB promoter mutation $(-70 \mathrm{bp}, \mathrm{C} \rightarrow \mathrm{A})$ \\
\hline GP2972 & GP1886 $(\Delta y b e C)$ & $17 \mathrm{mM}$ serine & hom promoter mutation $(-56 \mathrm{bp}, \mathrm{C} \rightarrow \mathrm{A})$ \\
\hline
\end{tabular}

The suppressor $\Delta$ serA 3 (GP2969) has a mutation in the gene thrR, encoding a transcriptional repressor of the genes of the threonine biosynthesis pathway (Rosenberg et al., 2016). The mutation is a missing base pair at position 90 of the gene and leads to a frameshift and finally to a very short version of the protein. This probably inactivated protein is no longer able to repress the promoters of $t h r D$ and of the hom-thrBC operon (Figure 45). These genes are involved in threonine biosynthesis and the inactivation of the repressor probably leads to an increase of threonine biosynthesis.
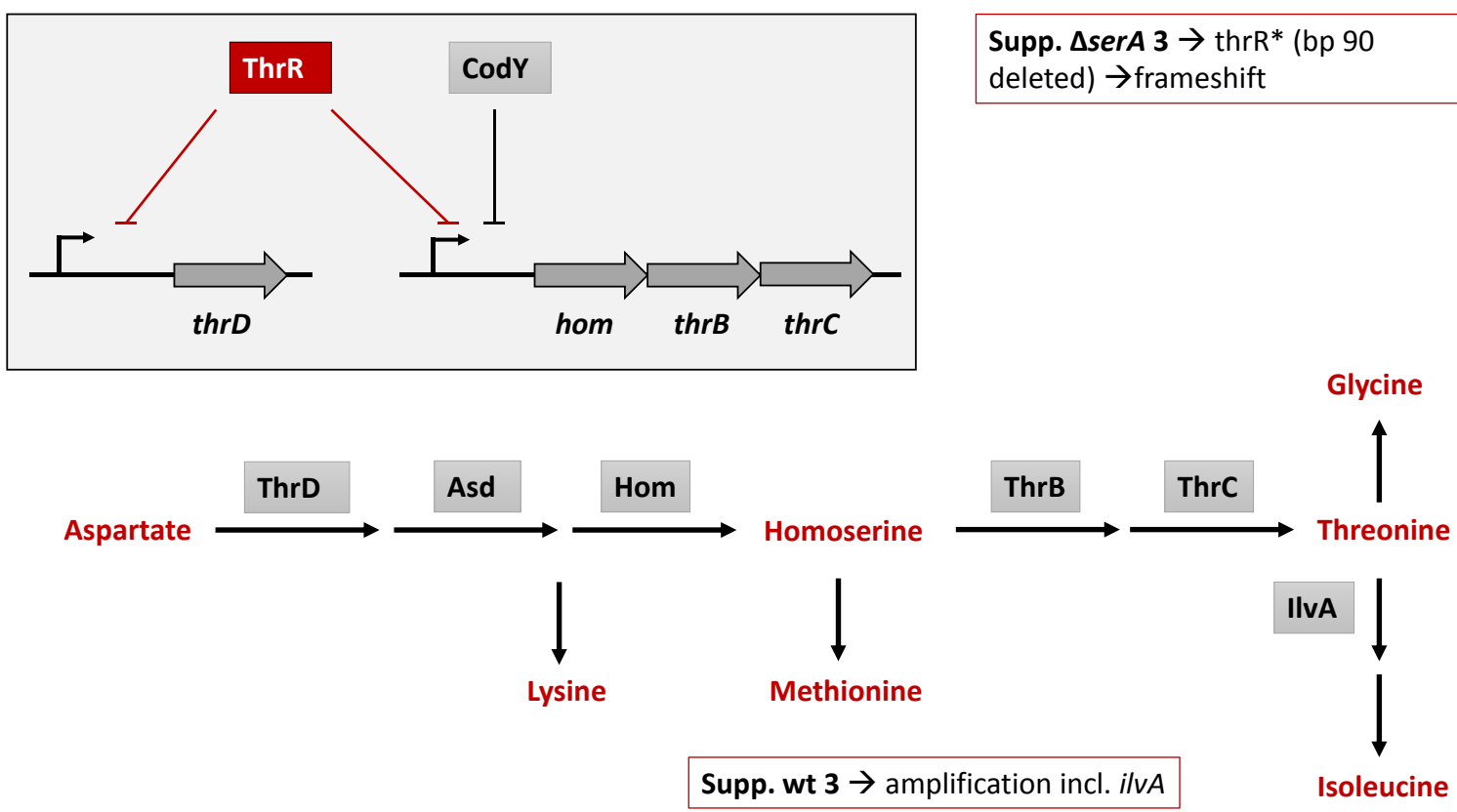

Figure 45: The suppressor mutations, that were found on high serine, are in genes that are involved in the threonine and isoleucine biosynthesis. The suppressor $\Delta$ serA 3 (GP2369) has a mutation in the transcriptional repressor gene thrR and the suppressor wt 3 (GP2970) shows amplification of the region including ilvA. 
The second suppressor wt 3 (GP2970) has no mutation, but a duplication of a genomic region of $15.7 \mathrm{~kb}$. This region includes 20 genes and shows a higher coverage in the WGS (Figure $46 \mathrm{~A}$ ). The region is duplicated and inserted into the ilvD gene. The insertion borders can be amplified in the strain GP2970 with the primer pairs AK356/357 and AK353/354 and thereby confirm the insertion (Figure 46 B). However, the ilvD gene can still be amplified in the wild type size. Furthermore, the coverage across the duplication region varies, since the genes yokABCD show an even 3-fold higher coverage of around 183 reads in comparison to the coverage to the rest of the genome (around 60 reads) (Figure $46 \mathrm{C}$ ). This indicates that the duplication or even 3 - fold amplification might be more complex.

A

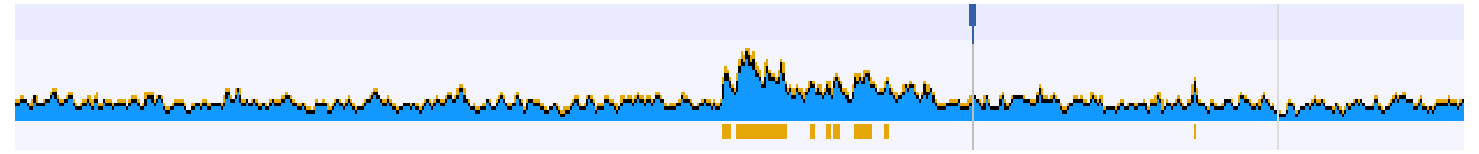

B
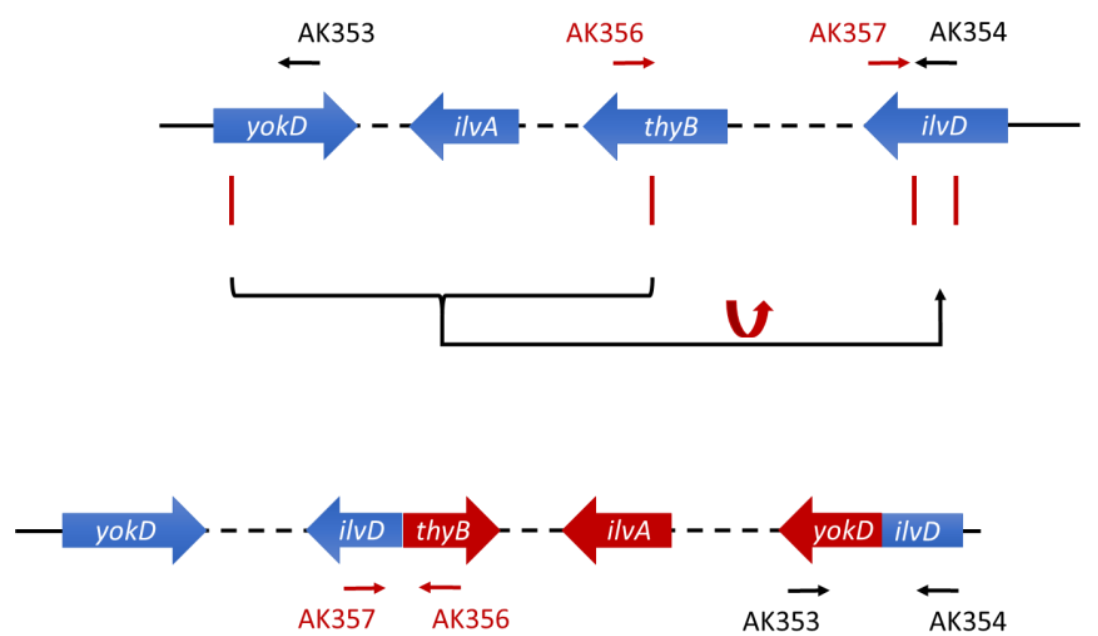

C

3-fold amplification (mean coverage 183)

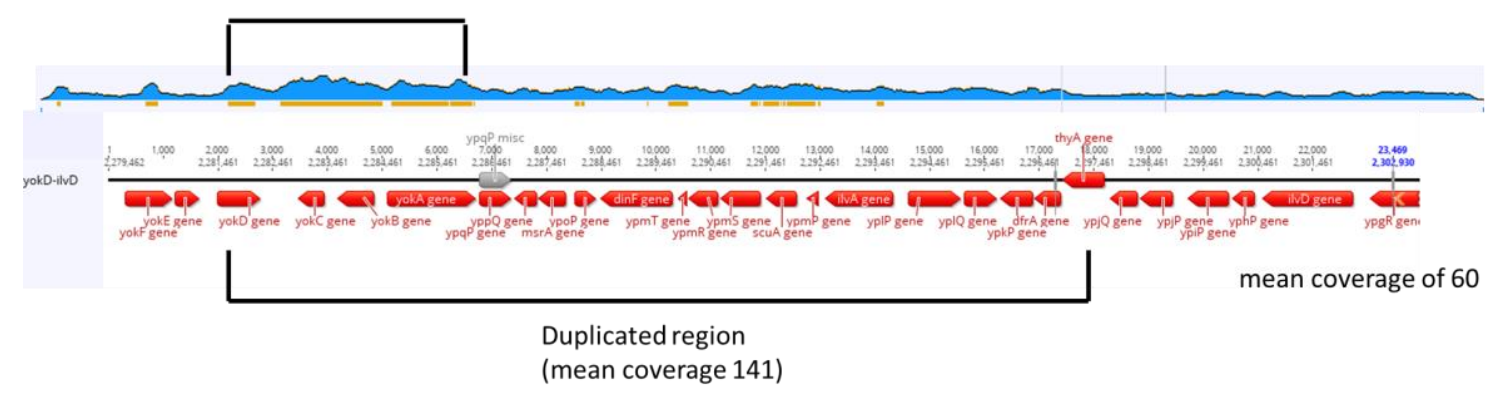

Figure 46: The duplication region in the suppressor mutant GP2970. A The duplication region was identified by WGS, since the coverage of reads is higher in this region. B The region from yokD to thy $B$ is duplicated and inserted into the ilvD gene. The designed primer pairs AK356/357 and AK353/354 can amplifiy the boarders in the suppressor mutant. C The coverage of the genomic region including yokABCD is even higher and indicates that an additional amplification of this region might be possible. 
The amplification region also includes ilvA, encoding the threonine dehydratase, which is involved in the production of isoleucine from threonine. To test, if the amplification of ilvA is the reason for the improved growth on C-glc plates with serine, an overexpression plasmid of ilvA was constructed (pGP2289). The gene ilvA was cloned into the vector pBQ200, which can be used for the constitutive overexpression. The plasmid pGP2289 was transformed into the wild type strain 168. This ilvA overexpression strain and a deletion mutant of thrR (BKE27910) (Koo et al., 2017) were compared with the growth of the suppressor mutants GP2969 and GP2970 (Figure 47).

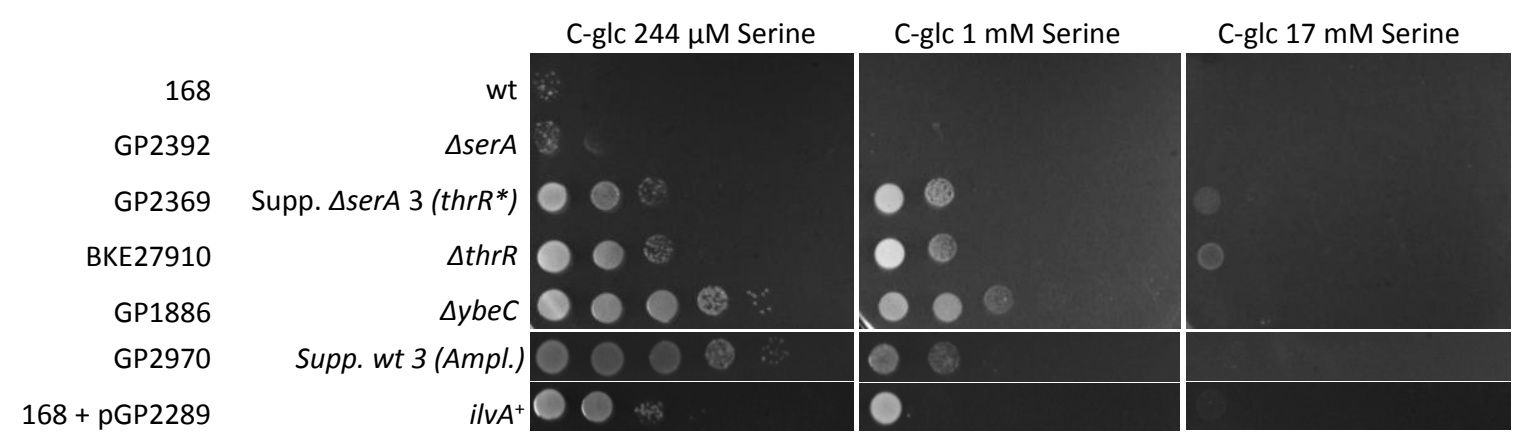

Figure 47: The drop dilution assay to test if the mutation in thrR and the amplification of the region including ilvA, influence the growth on C-glc serine medium. The mutations were found as suppressor mutants on high serine concentrations and are used as a comparison. The plates were incubated two days at $37^{\circ} \mathrm{C}$.

It can be observed, that the suppressor $\Delta$ serA 3 (GP2369) shows the same growth as the $\Delta t h r R$ deletion mutant. This leads to the assumption that the inactivation of ThrR results in a derepression of the genes involved in the threonine pathway and finally in a growth advantage on high serine concentrations. The overexpression of ilvA leads also to a growth advantage on C-glc serine plates, but the suppressor wt 3 (GP2970) grows even better. This probably means that the amplification of another gene of the amplified region is also beneficial, beside ilvA. The genes in the amplified regions are listed in Table 13. Many unknown genes can be found in the amplification region. However, further analysis needs to be done. It is also possible, that a protein with a known function, has a second minor activity that rescues the serine toxicity phenotype. This was already observed for the threonine synthase ThrC, which has a minor threonine dehydratase activity (Rosenberg et al., 2016).

The growth of the $\Delta t h r R$ mutant and the ilvA is not as good as the growth of the $\Delta y b e C$ mutant (Figure 47). The reduction of serine import seems to be of more advantage than deleting the repressor thrR. This indicates, that ThrR might not be the target of serine in the cell. To exclude a potential regulation of ThrR by serine, the expression of the genes, regulated by ThrR are analysed in the presence and absence of serine (Table 14). The strains BP558 and BP562 harbour a promoter lacZ fusion of the hom and the thrD promoter, integrated of into the amyE locus of the wild type 
strain 168. The construction is based on the pAC5 plasmid. Furthermore, the strains BP557 and BP563 also harbour these hom and thrD promoter lacZ fusion constructs, but in a strain with an additional deletion in thrR (Rosenberg et al., 2016).

Table 13: The genes of the amplification region including ilvA

\begin{tabular}{|l|l|}
\hline Gene & Description of the function \\
\hline yokD & Unknown \\
\hline yokC & Unknown \\
\hline yokB & Unknown \\
\hline sprA & Excision of the SP-beta prophage \\
\hline ypqP & Spore envelope polysaccharide biosynthesis \\
\hline$m s r B$ & Regeneration of methionine, restoration of protein function after oxidative damage \\
\hline$m s r A$ & Regeneration of methionine, restoration of protein function after oxidative damage \\
\hline$y p o P$ & Unknown \\
\hline$y p n P$ & Unknown \\
\hline$y p m T$ & Unknown \\
\hline$y p m S$ & Unknown \\
\hline$y p m R$ & Unknown \\
\hline$s c o$ & Maturation of cytochrome c oxidase caa3 \\
\hline$y p m P$ & Unknown \\
\hline$i l v A$ & biosynthesis of branched-chain amino acids \\
\hline$y p I P$ & required for survival at low temperatures \\
\hline$y p k Q$ & Unknown \\
\hline$y p k P$ & Unknown \\
\hline$d f r A$ & biosynthesis of folate \\
\hline$t h y B$ & biosynthesis of thymidine nucleotides \\
\hline
\end{tabular}

Table 14: The $\beta$-galactosidase activities of the hom and thrD promoters under the availability of serine

\begin{tabular}{|c|c|c|c|c|}
\hline & Strain & & C-glc & C-glc $244 \mu \mathrm{M}$ serine \\
\hline \multirow{4}{*}{$\begin{array}{l}\beta \text {-galactosidase activity } \\
\text { [Miller units] }\end{array}$} & $\mathbf{P}_{\text {hom }}$ & (BP558) & $283.1 \pm 42.6$ & $302.8 \pm 46.6$ \\
\hline & $P_{\text {hom }} \Delta t h r R$ & (BP557) & $1427.8 \pm 239.2$ & $1423.9 \pm 175.2$ \\
\hline & $\mathbf{P}_{\text {thrD }}$ & (BP562) & $148.6 \pm 0.9$ & $146.6 \pm 15.6$ \\
\hline & $\mathbf{P}_{t h r D} \Delta t h r R$ & (BP563) & $1502.7 \pm 284.4$ & $1394 \pm 226.1$ \\
\hline
\end{tabular}


The activity of the promoter from the hom operon and the thrD promoter were analysed in C-glc medium with and without serine. There is no difference in promoter activity detectable in both strains if serine is added to the medium. The deletion of thrR leads to an increase of the hom operon and thrD expression. This can also be observed here, but the addition of serine also leads to no change in expression. These results indicate that the expression of both promoters is not directly influenced by serine and that the activity of the repressor ThrR is not regulated by serine. To conclude, ThrR is not the target of serine toxicity in $B$. subtilis.

The suppressors GP2971 and GP2972 derive from the ybeC deletion strain and were isolated on 10 and $17 \mathrm{mM}$ serine. The suppressor strain GP2971 exhibits a mutation in the promoter of the operon $s d a A B-A A$, which encodes the L-serine deaminase. This protein is involved in the serine utilization by converting serine to pyruvate (Xu and Grant, 2013). The suppressor (GP2972) has a mutation in the hom operon promoter, that was already mentioned. Interestingly, the mutation is located in the binding side of the transcriptional repressor ThrR (Figure 48).

A

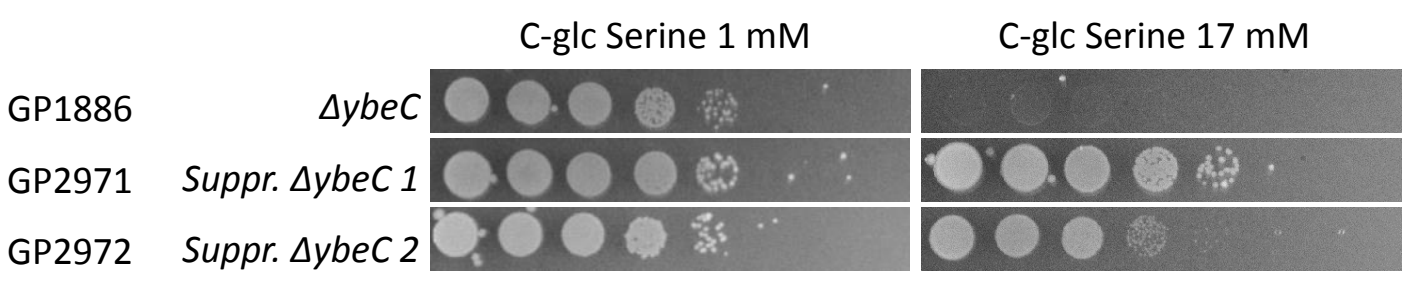

B

sdaAB promoter (GP2971)

Mutation

$C \rightarrow A$

CCCGCTTGCCATTTTATATCTGATCAATTAGTCTAGAAGCAAAGCGCTAATAAGCTCTGGTTCATAAGGAGGAATAGCATGAAA

sdaAB start

hom promoter (GP2972)

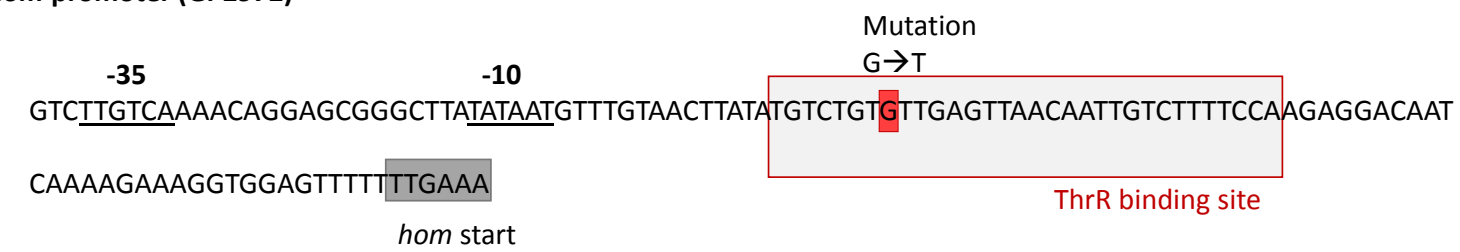

Figure 48: The suppressor mutations in the promoter regions of $s d a A B-A A$ and the hom operon. A The Drop dilution assay of the suppressor mutants GP2971 and GP2972 in comparison to the parental strain GP1886 B The suppressor mutations can be found in the promoter regions of the sdaAB-AA operon and the hom operon. Furthermore, the mutation in front of the hom gene is in a binding site of the transcription repressor ThrR. 
To analyse the effect of the mutation on the expression of the genes, lacZ fusions of the $s d a A B$ and the hom promoters, with and without mutations, were constructed. The strain BP558 has a native hom promoter lacZ-fusion integrated into the amyE locus. The primerst MT24 and MT25 that were used for the construction of BP558, were used in this work to amplify the hom promoter with the mutation in the ThrR binding site (Rosenberg et al., 2016). This mutated hom promoter was cloned into the vector pAC5, to construct the plasmid pGP2296, which was further transformed into the strain 168 (GP2968). The native and the mutated $s d a A B$ promoter was amplified with the primers AK379/380 and cloned into the pAC5 plasmids. The plasmids pGP2294 (sdaAB mutated) and pGP2295 (sdaAB native) were transformed into the wild type strain 168 to construct strains GP2966 (sdaAB mutated) and GP2967 (sdaAB native). The activity of these translational promoter-lacZ fusions was measured with a $\beta$-galactosidase activity. The strains was grown in different media to an $\mathrm{OD}_{600}$ of 0.5 and the $\beta$-galactosidase activity was measured as described in chapter 2.2.7. (Table 15).

Table 15: The $\beta$-galactosidase activity assay to analyse the influence of the promoter mutations on the expression of $s d a A B$ and hom

\begin{tabular}{|l|l|l|l|l|}
\hline Promoter & $\begin{array}{l}\mathbf{P}_{\text {hom }} \\
\text { (BP558) }\end{array}$ & $\begin{array}{l}\mathbf{P}_{\text {hom }} \text { * G56T } \\
\text { (GP2968) }\end{array}$ & $\begin{array}{l}\mathbf{P}_{\text {sdaAB }} \\
\text { (GP2967) }\end{array}$ & $\begin{array}{l}\mathbf{P}_{\text {sdaAB }} \text { * C70A } \\
\text { (GP2966) }\end{array}$ \\
\hline $\begin{array}{l}\text { B-galactosidase } \\
\text { activity [Miller units] }\end{array}$ & $273.6 \pm 34.3$ & $973.3 \pm 83.2$ & $7.4 \pm 2.2$ & $368.4 \pm 47.8$ \\
\hline
\end{tabular}

These measurements show that the mutations of the promoter region of each operon lead to an increase in the expression. For the $s d a A B-A A$ operon, these results indicate, that more copies of the L-serine deaminase are in the cell and more serine is converted to pyruvate. The toxic effect of serine is compensated by reducing the amount of serine in the cell. As already mentioned, the mutation in the hom operon promoter is in the binding site of the repressor ThrR and leads probably to a reduced binding of the protein to the DNA. Therefore, the expression of the promoter is not repressed and more enzymes from the threonine biosynthesis pathway are produced.

To summarize, two kinds of suppressor mutations could be identified for the growth on high serine concentrations. The first one leads either to the reduction of serine uptake by mutating the importer YbeC or to an increase of serine utilization by the upregulation of the $s d a A B-A A$ expression. The second kind of mutation is the upregulation of threonine and isoleucine biosynthesis. Interestingly, the threonine biosynthesis is often upregulated by directly inactivating the repressor ThrR or by mutating the DNA binding targets of this protein. This link to the biosynthesis of threonine and especially to the hom-thrB-thrC operon is interesting, since it is known for E. coli, that serine can inhibit the homoserine dehydrogenase (ThrA) (Hama et al., 1990). 
However, also the genes thrB and thrC and their products could be potential targets. In B. subtilis, some amino acids can compensate the toxic effect of serine, if they are added additionally to the medium. Threonine, glycine, methionine, tryptophan, tyrosine are described to compensate low concentrations of $125 \mu \mathrm{M}$ serine and furthermore, alanine, arginine, aspartate, glutamate and proline can compensate even $500 \mu \mathrm{M}$ of serine. However, not all amino acids were tested for their potential compensation (Lachowicz et al., 1996). The compensatory effect of some amino acids would also give another hint to whether the threonine pathway is affected by the serine toxicity. A drop dilution assay was performed with the wild type strain 168 on medium with 244 or $500 \mu \mathrm{M}$ serine and one additional amino acid in a concentration of $500 \mu \mathrm{M}$ (Figure 49).

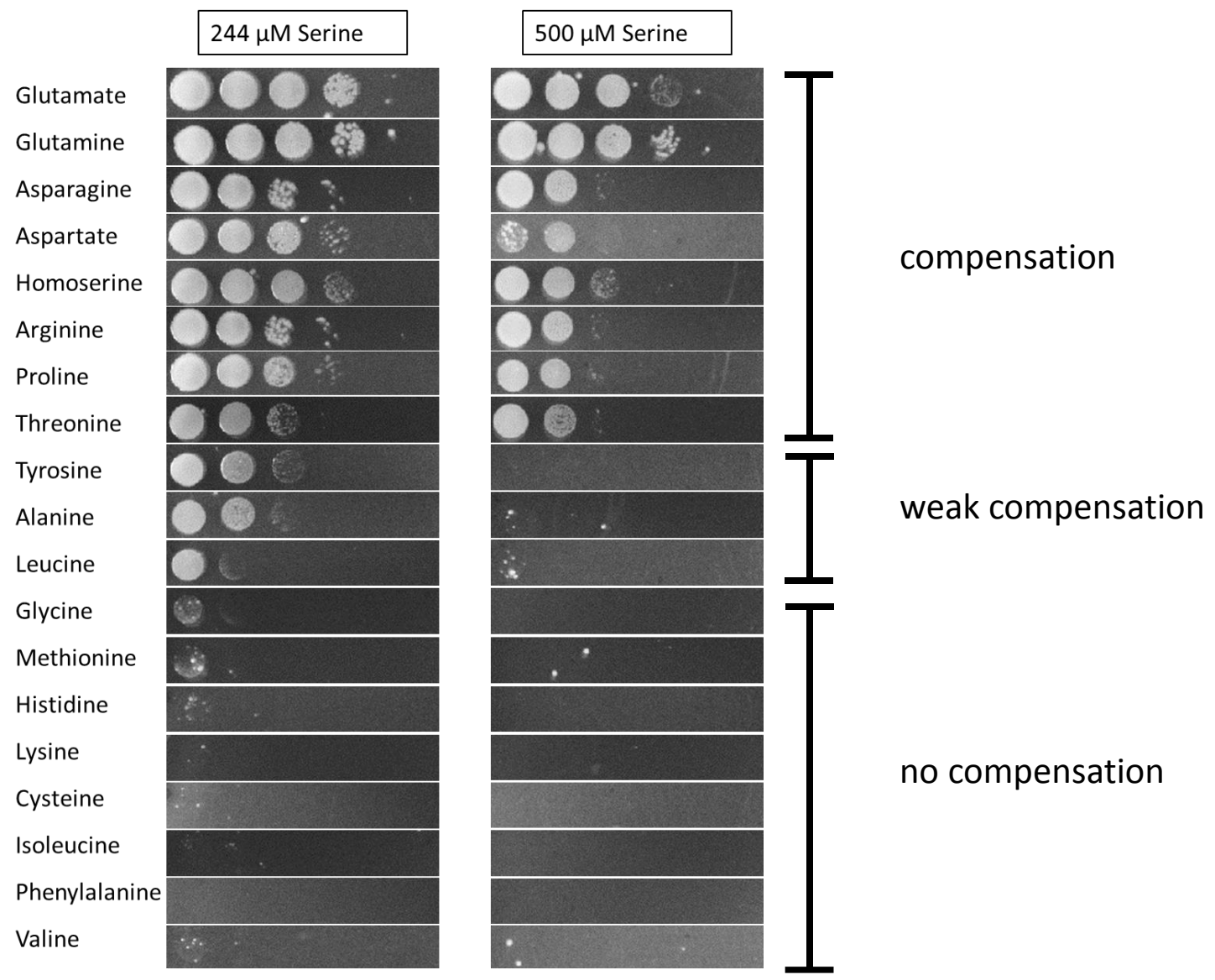

Figure 49: The drop dilution assay to test which amino acids can compensate the toxic effect of serine. The effect of $500 \mu \mathrm{M}$ of each amino acid on the growth of the wild type strain 168 was analysed on plates with each $244 \mu \mathrm{M}$ or $500 \mu \mathrm{M}$ serine.

The addition of some amino acids lead to the compensation of the toxic effect of serine, since the wild type is able to grow on these plates. However, for some amino acids (tyrosine, alanine and leucine) the compensation is only possible with the low concentration of serine $(244 \mu \mathrm{M})$ and some amino acids are not able to compensate the effect of serine. It was previously mentioned (chapter 3.3.3.) that the compensation could be also due to the import of the amino acid by the same transporter as serine. The obtained results were illustrated in Figure 50. 
Interestingly, aspartate and asparagine were detected to compensate the toxic serine effect. Aspartate is a precursor of the threonine biosynthesis and a high amount of it in the cell could also lead to a higher production of threonine. The addition of glutamate, glutamine, asparagine and proline might also lead to the same effect, because glutamate can be converted to $\alpha$-ketoglutarate, an intermediate of the TCA cycle. Aspartate can then be synthesized from oxaloacetate. Furthermore, high glutamate concentrations are also needed for the transaminase reactions, that are involved in all amino acid biosynthesis pathways.

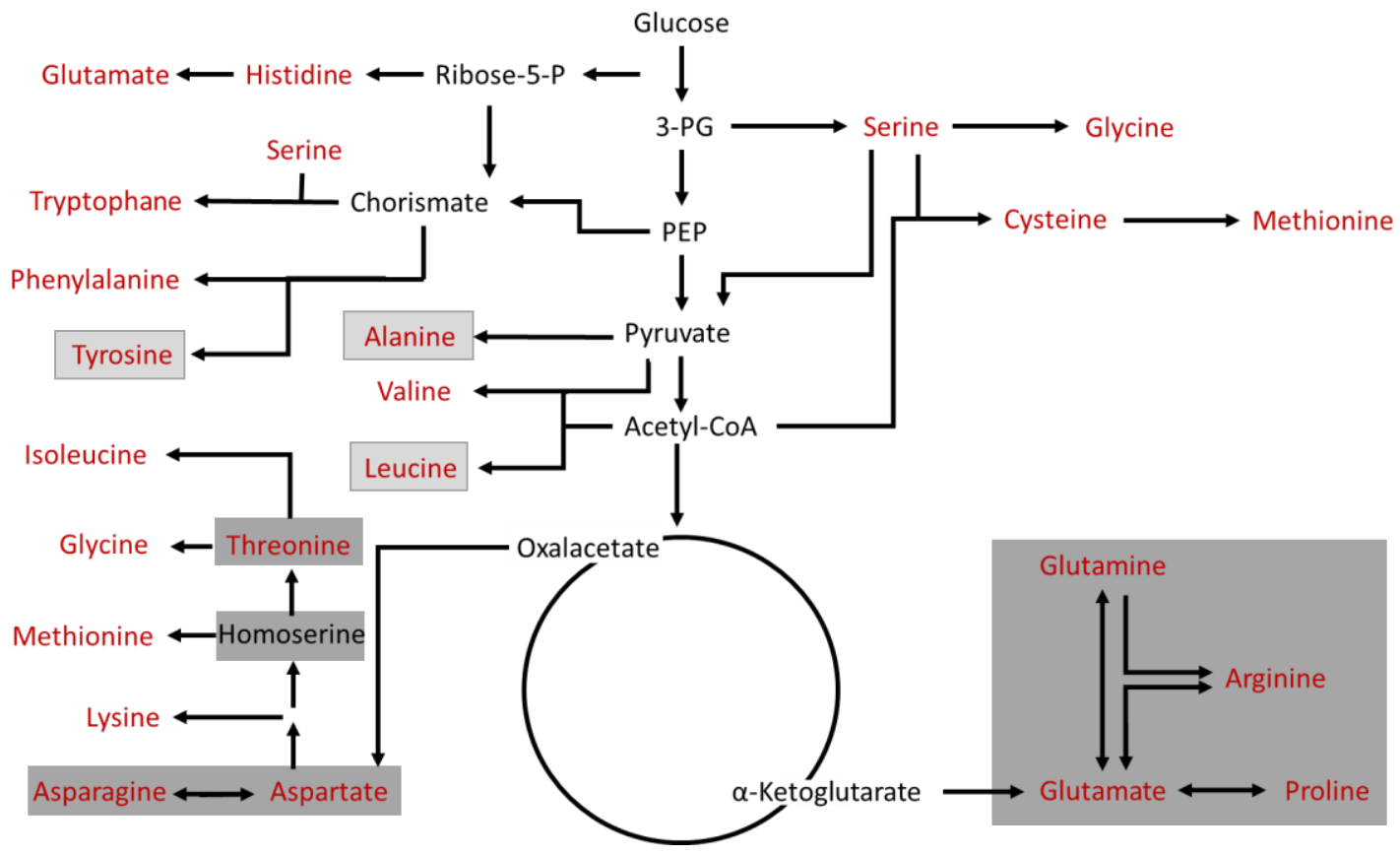

compensation

weak compensation

Figure 50: The amino acid biosynthesis pathways in B. subtilis. The amino acids that can compensate the toxic effect of serine are marked in grey.

It has to be noticed, that these mentioned amino acids were already detected in the screening for threonine importers (chapter 3.3.3.). Therefore, it is also possible, that some of these amino acids are also transported into cell by one of the serine/ threonine transporters. Furthermore, also threonine was detected, which could also be an effect of the shared transporter with serine. However, beside all amino acids, also homoserine was tested for its compensatory effect. Homoserine is formed by the homoserine dehydrogenase Hom and if this enzyme is affected by the serine concentration, the addition of homoserine can compensate the toxic effect. The obtained results support this theory, that the enzyme activity of the Hom protein must be altered by serine. To test this hypothesis, a Hom enzyme activity assay was performed. The reverse reaction of the homoserine dehydrogenase from L-homoserine and $\mathrm{NADP}^{+}$to L-aspartate 4-semialdehyde and $\mathrm{NADPH}$ was measured by the conversion of $\mathrm{NADP}^{+}$to $\mathrm{NADH}$ and the resulting change in absorbance 
at $340 \mathrm{~nm}$. The Hom protein was overexpressed from a plasmid in E. coli and purified. The first version of the protein was purified with a N-terminal SUMO-tag that could be cleaved by the SUMO protease (pGP2297). However, the cleavage was always only successful for half of the proteins and the protein solution showed no activity in vitro. As a second attempt a C-terminal strep-tagged construct was designed, but unfortunately this enzyme was also not active in vitro (data not shown).

Although it was not possible to measure the Hom activity in vitro and furthermore the serine influence on it, the results suggest a regulatory role of serine on the Hom activity. Some of the suppressor mutants that were isolated showed a link to the threonine pathway. It could be excluded that the transcriptional repressor ThrR of the threonine pathway is the serine target. Nevertheless, the operon hom-thrBC seems to be involved. The compensatory effect of homoserine on the serine toxicity strongly suggest, that the target of serine inhibition is the Hom enzyme. However, it could be shown, that the expression of the hom-thrBC operon is not influenced by the presence of serine.

\subsection{The consequences for the MiniBacillus project (Blueprint 2.0)}

The genes $y b e c, y b x G$ and $b c a P$ were identified to encode for serine/ threonine transporters. However, these are not the only serine and threonine importers in B. subtilis. The blueprint of the desired MiniBacillus strain includes the known amino acid importers, instead of the biosynthesis pathways. However, importers for serine were previously unknown in B. subtilis, therefore the biosynthesis pathway of serine was annotated to remain in the MiniBacillus strain (Reuß et al., 2016). But the results of this work allow to keep one of the importers of serine, instead of the biosynthesis pathway. Although, $\mathrm{YbeC}$ seems to be the major serine importer, there is no need for this protein to remain in the final MiniBacillus strain. An increased concentration of serine in the cell leads to the inhibition of the threonine pathway. In theory, one of the minor serine importers YbxG or BcaP should import enough serine into the cell to sustain good growth. The transporter BcaP will remain in the MiniBacillus strain anyway, since the transport of valine, isoleucine and also threonine is facilitated by this protein. To analyse if BcaP alone is able to sustain growth in LB-glc, growth curves were performed (Figure 51). The effect of the deletion of $y b e C$ and $y b x G$ was tested to confirm that the cell can survive with BcaP as the only known serine/ threonine transporter. Since the LB medium provides a lot of amino acids, the strains grow all similar. Even the combination with a $\Delta s e r A$ or $\Delta t h r C$ mutant leads to no change in growth. These additional deletions were done to mimic the situation in the final MiniBacillus strain, which will be auxotrophic for most amino acids. To conclude, although we do not know all serine transporters in $B$. subtilis, the protein BcaP will probably import enough serine to sustain growth. The deletion of the major serine importer YbeC will probably have no effect on the growth of the strain. Furthermore, the current MiniBacillus 
strain PG39 has already deleted $y b e C$ and $y b x G$. However, the biosynthesis gene for serine are still in the genome, but the obtained results indicate, that the deletion of those will not lead to a serine shortage.

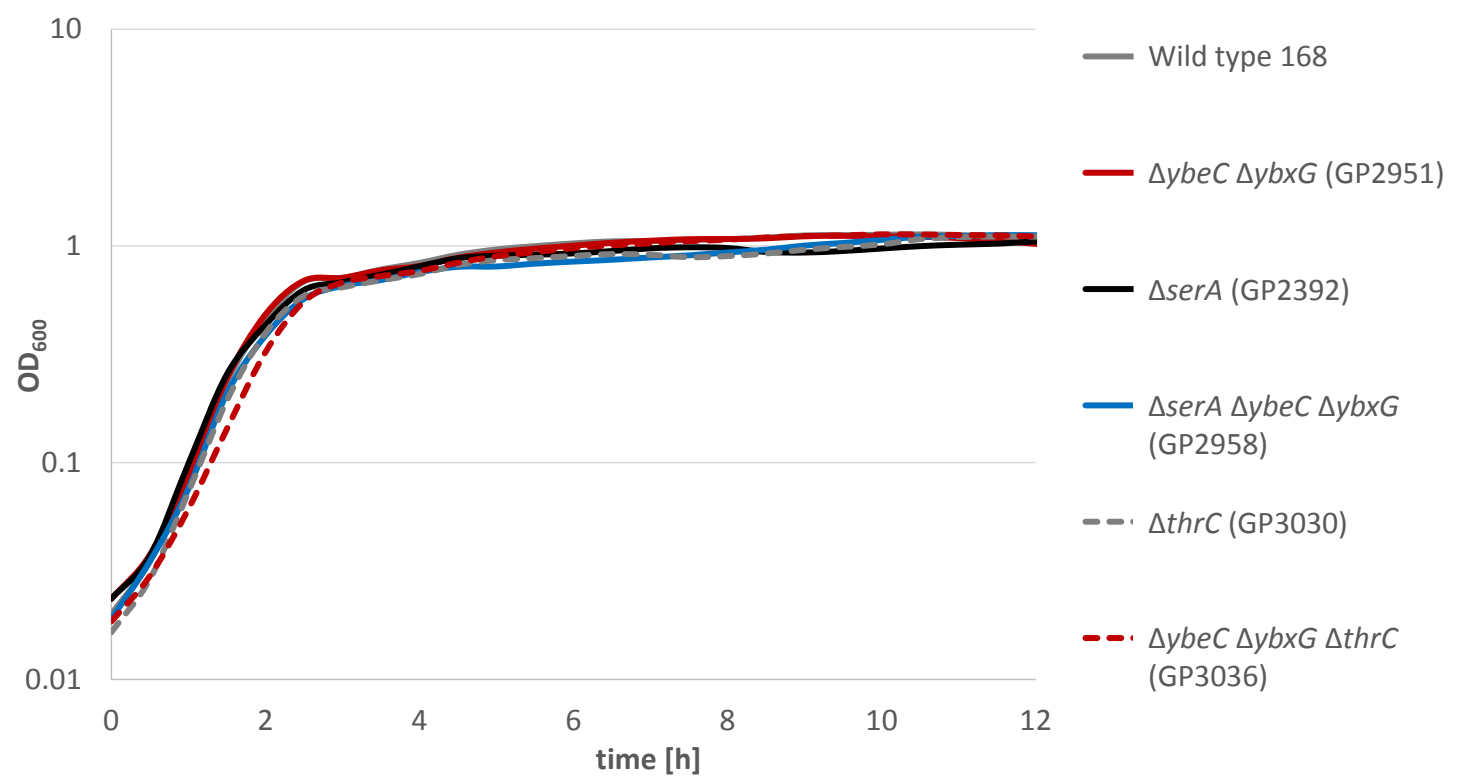

Figure 51: Growth curves in LB-glc medium to test if the lack of ybeC and ybxG might lead to a growth defect. The serA deletion leads to a serine auxotrophic strain and the $t h r C$ deletion to a threonine auxotrophic strain. The strains show no growth differences.

The results of this work can now contribute to an updated version of the MiniBacillus design, the blueprint 2.0. The genes encoding for the serine biosynthesis serA, serB and serC can be deleted and $b c a P$ remains as the isoleucine, valine, threonine and serine importer. Furthermore, new data from e.g. the publication by Koo et al. (2018) can be included. They revealed new insights about the essentiality of genes by creating two deletion mutant libraries. Furthermore, the competence of the constructed deletion strains was tested, which is also from high importance for the MiniBacillus project. The number of genes in each category of the blueprint 2.0 are shown in Table 16.

Table 16: The comparison of the blueprint 1.0 and 2.0

\begin{tabular}{|l|c|c|c|c|}
\hline Function & \multicolumn{2}{|c|}{ Blueprint 1.0 } & \multicolumn{2}{c|}{ Blueprint 2.0 } \\
\hline & Protein genes & RNA genes & Protein genes & RNA genes \\
\hline Information & $197(125)$ & $119(2)$ & $201(131)$ & $119(2)$ \\
\hline Metabolism & $218(59)$ & & $215(74)$ & \\
\hline Cell division & $81(52)$ & & $84(57)$ & \\
\hline Integrity of the cell & $16(5)$ & & $17(6)$ & \\
\hline Other/ unknown & $11(2)$ & & $16(7)$ & \\
\hline Total & $523(243)$ & $119(2)$ & $537(275)$ & $119(2)$ \\
\hline
\end{tabular}


The total number of protein coding genes increases to 537 genes, from which 275 are essential. However, the number of RNA coding genes remains the same. The complete list of all included genes can be found in the supplementary information (chapter 6.5.). This new version of the MiniBacillus blueprint and the results of this work can contribute to improve the deletion process. 


\section{Discussion}

\subsection{The MiniBacillus project}

One approach to understand the complexity of life is to reduce it. Several attempts in different organisms were performed. Especially, the minimal genome strains of $B$. subtilis show enhanced protein productivity and are therefore interesting for biotechnological applications (Morimoto et al., 2008; Aguilar Suárez et al., 2019). The MiniBacillus project attempts to create a minimal B. subtilis cell, in which every gene has an assigned function (Reuß et al., 2016). An important goal in this project is to gain more knowledge about the organism and to utilize this information again to adapt the deletion process. During this work, a MiniBacillus strain with a genome reduction of $40.51 \%$ was created, which is the most extensive described reduction in a top-down approach and also for the model organism B. subtilis. The strain PG39 is still stable and shows good growth in the standard conditions LB-glc medium at $37^{\circ} \mathrm{C}$. However, the maximal biomass that is reached in the growth curves is slightly reduced. But since the overall growth is stable, this will probably have no disadvantage for the MiniBacillus project. A slightly reduced growth of a genome reduced $B$. subtilis strain was already observed before, but this had no effect on the protein productivity (Ara et al., 2007). Similarly, the strain PG39 could also show a similar or even better protein productivity than the ancestor strain PG10, although PG39 cannot reach the final biomass of PG10 in LB-glc medium (Aguilar Suárez et al., 2019).

The multi-omics analysis is very important for the project, since it provides a multi-layered picture of the situation in the cell. Especially the transcriptome data has a high impact, since changes in the expression of genes caused by the deletions can be identified. The operons paiAB and mhqNOP were shown to be higher expressed in the strain PG10 in comparison to the ancestor strain $\Delta 6$ (Reuß et al., 2017). Since PG10 was the last strain to be analysed with the multi-omics approach, a new set of data for the current strains would provide new insights. The proteome of the strain PG38 was analysed during this work. The data indicate, that the proteome of PG10 and PG39 are very similar. There is no evidence for the overexpression of a certain pathway. To get insights into every aspect of the strain and to ensure a stable strain, a complete multi-omics data set is necessary.

The described point mutation in the low-affinity phosphate transporter gene pit, lead to a high impact in the MiniBacillus strain (Reuß et al., 2017). The phosphate level in the cell decreased and the PhoPR system was activated. This leads to the activation of genes for the acquisition of phosphate, like the phosphate $A B C$ transporter for the high-affinity uptake of phosphate. On the other hand, the operons $\operatorname{tag} A B$ and $\operatorname{tag} D E F G H$ for the biosynthesis of teichoic acid are repressed (Allenby et al., 2005). This might have caused the observed mutations in the tagAB promoter and 
in the gene yqgS, encoding for the minor lipoteichoic acid synthase to counteract the constant regulation by the PhoPR system (chapter 3.1.3). Similarly, the phosphodiesterase / alkaline phosphatase PhoD would also be higher expressed in the strain PG18 with the pit mutation. PhoD is active during phosphate starvation conditions and degrades wall teichoic acid (Eder et al., 1996). This could lead to an unwanted damage of the cell wall. Because of this reason, the analysis of the MiniBacillus strains by WGS is very important to detect such point mutations and to adapt the deletion process, before counteracting suppressor mutations accumulate.

A main problem of the MiniBacillus project is, that the deletion process becomes more difficult, since the deletion regions become smaller and thereby the deletion process decelerates. A solution for this might be the defragmentation approach to cluster functionally related genes together in one locus. The insertion of the glycolytic cassette shows, that the defragmentation approach in general is possible and that it could increase the deletion process. The clustering of functionally related genes was already used in metabolic engineering (Qi et al., 2015). Metabolic engineering enables the construction of customized strains (Oesterle et al., 2017). Such strains would be beneficial for biotechnology, since they can be easily modified, if a certain pathway should be deleted to increase the production of a certain product. The expression of certain pathways can also be regulated easily. But metabolic engineering is a complicated process, since the transcriptional units need to be considered. Some genes can be transferred with their natural promoter or terminator, but some genes have to be fused to a new promoter or terminator to sustain the correct transcription. If an artificial promoter is used, it has to maintain a certain level of expression and also a similar regulation that was given by the natural promoter. Otherwise the different regulation will lead to an imbalance in the cell. As an example, the higher expression of the serine biosynthesis genes, would lead to a growth inhibition by the increasing serine concentrations (Lachowicz et al., 1996). Furthermore, the insertion of the modified DNA segments needs to be tested for functionality, before the natural locus is deleted. Several B. subtilis production strains were already constructed by metabolic engineering. By the insertion of several genes, a $B$. subtilis strain was created, that is able to produce D-lactic acid, a compound of poly lactic acid (Awasthi et al., 2018). However, it is questionable if the defragmentation approach provides the final solution for the decrease of the deletion process.

A genomic approach of metabolic engineering is represented by the bottom-up genome reduction strain JCVI-syn3.0. The strain is synthesized. A first approach with a set of genes, which are known to be necessary for the strain, was unsuccessful. Therefore, the genome of the final minimized strain still contains 149 genes with unknown function (Hutchison et al., 2016).

Since a main goal of the MiniBacillus project is to gain more knowledge about the cell, this work focuses additionally on the analysis of different pathways, like the TCA cycle or the 
biosynthesis and uptake of amino acids. The results of this work and the new information from several publications were incorporated to a new blueprint 2.0, based on the original blueprint of a minimal cell (Reuß et al., 2016). Especially the work of Koo et al. (2017) identified many new essential genes and competence genes, which are important for the MiniBacillus strains. Furthermore, the missing gene of the biosynthesis of serine was discovered to be $y s a A$, which was therefore renamed to serB. The constant adaptation and re-evaluation of the blueprint is necessary to improve the deletion process. Similarly, the characterization of genes with unknown function is important to reduce the risk of dead ends in the MiniBacillus project.

\subsection{The functions of the two citrate synthases}

The role of the two citrate synthases of $B$. subtilis is unknown. They are differently regulated and the deletion of citZ has a greater impact on the growth in C-malate medium than the deletion of citA (chapter 3.2.1.) (Jin and Sonenshein, 1994a). The reaction of the citrate synthase is important, since it provides an important link to glycolysis (Jin and Sonenshein, 1994b). This could mean that the two citrate synthase enzymes catalyse the same reaction under different conditions to sustain a certain level of influx into the TCA cycle. Interestingly, the expression of the aconitase CitB and the isocitrate dehydrogenase gene Icd, which convert citrate further, are also regulated like the major citrate synthase CitZ (Jourlin-Castelli et al., 2000; Kim et al., 2002). The expression is repressed in the presence of glucose and glutamate. This means that the citrate which is produced under these conditions by the minor citrate synthase CitA, cannot be metabolized further. This would lead to an increase in the intracellular citrate concentration, which was shown to affect the ability to sporulate and is therefore a disadvantage for the cell (Craig et al., 1997).

The activity assay of the two citrate synthases shows that the major citrate synthase CitZ can form more citrate than the minor citrate synthase CitA. This leads to the assumption, that oxaloacetate and acetyl-CoA might not be the ideal substrates for the CitA enzyme. Another paralogous protein of the citrate synthases is annotated in the genome of $B$. subtilis, the 2methylcitrate synthase MmgD (Reddick and Williams, 2008). This enzyme catalyses the reaction of propionyl-CoA and oxaloacetate to 2-methylcitrate and $\mathrm{CoA}$, which is similar to the citrate synthase reaction (Reddick et al., 2017). The enzyme is part of the methylcitric acid cycle to produce pyruvate from propionate. For organisms like E. coli, this metabolic pathway is important to grow with propionate as the single carbon source (Brock et al., 2002). In B. subtilis, the gene $m m g D$ is encoded in the $m m g$ operon, which is part of the mother cell metabolism. This operon is only active at a certain time during sporulation, mediated by the sigma factor $\sigma^{\mathrm{E}}$ controlled promoter. Additionally, the promoter is controlled by carbon catabolite repression through $\operatorname{CcpA}$, which leads to a repression in the presence of glucose. The enzyme MmgD shows also citrate synthase activity 
(Bryan et al., 1996; Reddick et al., 2017). This could mean that the citrate synthase CitA also favours other substrates and is therefore involved in another metabolism. This would also explain the observation, that CitA can only partially compensate the loss of the major citrate synthase CitZ.

Interestingly, even three citrate synthase genes were identified in Saccharopolyspora erythraea, a species of actinobacteria (Oliynyk et al., 2007). The first gene gltA-2 is highly similar to the citZ gene from B. subtilis and it is similarly repressed by a regulator of carbon catabolite repression. In contrast, the genes citA and citA4 from S. erythraea are similar to the citA gene from B. subtilis. The citA and citA4 genes are transcriptionally repressed by $G \ln R$, a regulator of the nitrogen metabolism and DasR, a regulator of the amino-sugar metabolism (Yao et al., 2014; Liao et al., 2014). These similarities to the genes of $S$. erythraea might hint to a related regulation in B. subtilis. The fact that glutamate stimulates the expression of citA in glucose containing medium could also hintto a regulation by the nitrogen metabolism. In contrast, the expression of citZ is even higher repressed in medium containing glucose and glutamate, than in medium with only glutamate (Jin and Sonenshein, 1994a). However, this topic needs to be further analysed to elucidate the main function of the CitA protein.

\subsection{The role of the TCA cycle in B. subtilis}

The TCA cycle is not only a central point of carbon metabolism. It also provides important links to nitrogen metabolism and to several amino acid pathways. This work provides evidence, that the enzymes involved in this pathway are furthermore linked to other important processes in the cell.

The deletion of the complete TCA cycle is possible in B. subtilis and the involved enzymes are not necessary for the growth in LB-glc medium. Other organisms, like Mycoplasma pneumoniae harbour no TCA cycle. This is due to the fact, that the host provides all nutrients, which are imported into the cell (Manolukas et al., 1988; Halbedel et al., 2007). Accordingly, the TCA cycle deficient B. subtilis strain was not able to grow in C-glc minimal medium. Only the addition of glutamate led to the restoration of growth, since the TCA cycle deletion strain is glutamate auxotroph (chapter 3.2.3.). The glutamate auxotrophy was already observed for the single deletion mutants of the citrate synthase and the aconitase (Jin and Sonenshein, 1994b; Craig et al., 1997).

Interestingly, the deletion of the TCA cycle led to a reduced competence of the strain. This link of the TCA cycle to the competence could be narrowed down to the deletion of the succinate dehydrogenase complex, encoded by $s d h C A B$. This complex has an additional function in the respiratory chain, since SdhC is part of the cytochrome b558 (Hederstedt and Rutberg, 1983; Baureder and Hederstedt, 2011). It was already mentioned, that a similar protein, the NADH dehydrogenase $\mathrm{Ndh}$, is also important for competence, since the deletion strain shows no transformants (Koo et al., 2017). In contrast, the single deletion strains of $s d h C, s d h B$ and $s d h A$ are 
transformable (Koo et al., 2017). The deletion of $s d h C$ has therefore not the same impact as the deletion of $n d h$, although both are involved in the electron transfer to menaquinone (Matsson et al., 2000). Furthermore, the $n d h$ deletion mutation facilitates the growth without a cell wall, as Lform cells. This was due to the reduction of oxidative stress (Kawaii et al., 2015). Similarly, other genes involved in the respiratory chain, like qoxB, encoding for the cytochrome aa3 quinol oxidase subunit I, also showed L-form cell formation (Santana et al., 1992; Kawaii et al., 2015). Interestingly, this phenotype could also be observed for the TCA deletion strain, if no carbon source is added to the medium. Due to the similarity to $\mathrm{Ndh}$ and the role in the respiratory chain, the deletion of $s d h C$ might be the reason for the observed phenotype. Furthermore, the deletion of the complete TCA cycle could also have an impact. The TCA cycle produces the major part of the reducing agents $\mathrm{FADH}_{2}, \mathrm{NADH}+\mathrm{H}^{+}$and $\mathrm{NADPH}+\mathrm{H}^{+}$, which are further used in the respiratory chain to produce the energy equivalent ATP (Nakamura et al., 2011) (Figure 52).

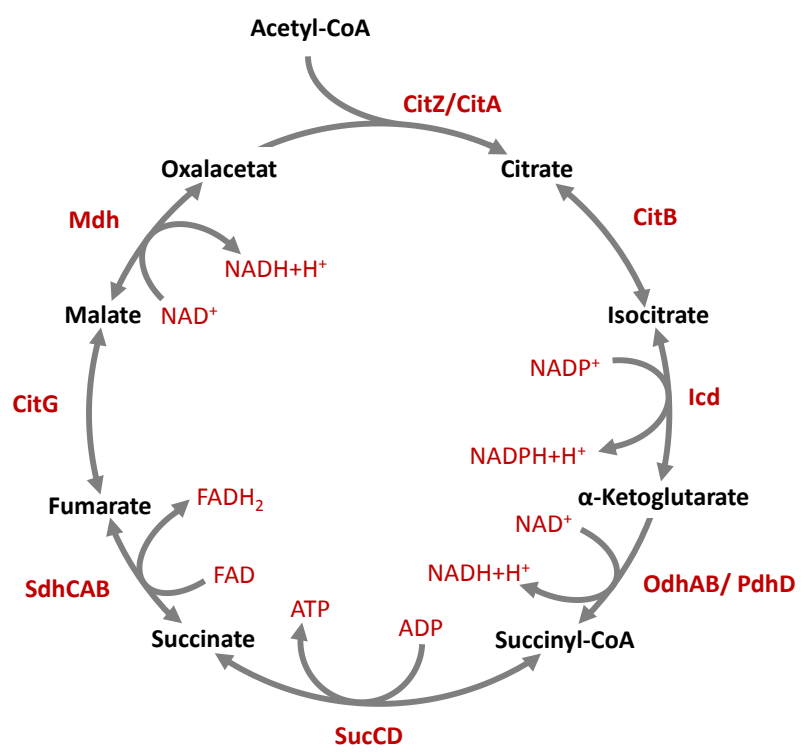

Figure 52: The TCA cycle of $B$. subtilis. The intermediates $\mathrm{NADH}+\mathrm{H}^{+}, \mathrm{NADPH}+\mathrm{H}^{+}, \mathrm{FADH} 2$ and ATP are produces in different steps in the TCA cycle.

If the TCA cycle does not operate, less reducing agents are produced, which leads to a decrease of the activity of the respiratory chain. This is necessary to promote L-form growth since the oxidative stress is reduced (Kawai et al., 2015). The addition of glucose leads to a the production of more $\mathrm{NADH}+\mathrm{H}+$ by the glycolysis, and thereby to an increase of the respiratory chain activity and the oxidative stress. This could prevent the formation of L-form cells. However, the L-form phenotype is probably combination of several effects. The observation that the addition of glucose can reverse the L-form formation indicates that the TCA cycle strain cannot produce enough peptidoglycan to sustain a stable cell wall. This might be due to the aspartate level in the cell. Aspartate can be formed from oxaloacetate, catalysed by the aspartate transaminase AspB (Dajnowicz et al., 2017). A deletion mutant of this enzyme is auxotrophic for aspartate and asparagine. The aspartate 90 
limitation leads to a decrease of 2-6-diaminopimelate (mDAP), a peptidoglycan precursor, which finally also reduces the peptidoglycan synthesis (Zhao et al., 2018). In the TCA cycle deletion strain, less oxaloacetate is produced and therefore less aspartate by AspB. This might be the reason for the decrease in peptidoglycan formation. In contrast, the addition of glucose leads to the increased production of pyruvate that can be further metabolised by the pyruvate carboxylase PycA to oxaloacetate. This leads again to an increase in aspartate and furthermore in an increase of peptidoglycan production. Additionally, the reaction catalysed by AspB is a transaminase reaction, which needs high amounts of glutamate (Zhao et al., 2018). Since the TCA cycle deletion mutant is also glutamate auxotroph, this might have also an effect on the production of aspartate. Interestingly, Mycoplasma cells have no TCA cycle and additionally no cell wall (Fraser et al., 1995). They do not produce peptidoglycan and the TCA cycle is not necessary as a link to this pathway.

Furthermore, the reduced peptidoglycan synthesis could also have an effect on sporulation. The TCA cycle deletion strain is not able to form spores. Since spores are coated with a layer of peptidoglycan, the lack of peptidoglycan might be one of the reasons for the blockage of sporulation (Tocheva et al., 2013). In previous studies, the block in sporulation was always narrowed to the accumulation of TCA intermediates, due to the deletion of single TCA cycle enzymes. The deletion of the aconitase CitB results in the accumulation of citrate, which builds chelating complexes with divalent ions like $\mathrm{Mn}^{2+}$ and $\mathrm{Fe}^{2+}$. These ions are used to initiate the SpoOA phosphorelay and thereby sporulation (Craig et al., 1997). This could also be shown for the icd mutant, but the addition of divalent ions and the lowering of the $\mathrm{pH}$ could lead to a complementation of the sporulation effect. Furthermore, the additional deletion of the major citrate synthase CitZ leads to a decrease of citrate in the cell and also to a compensation of the sporulation defect (Matsuno et al., 1999). Since the complete TCA cycle was deleted, no intermediates should have accumulated that lead to a block in sporulation. However, the TCA cycle deletion strain is still unable to form spores and the reason for that still needs to be further investigated.

Spo0A plays also as role in genetic competence (Mirouze et al., 2012). The level of phosphorylated SpoOA (SpoOA*) in the cell is an important indicator for the cell faith. At low SpoOA* concentrations the competence is inhibited, since ComK is repressed by the AbrB-Rok mechanism. At a certain intermediate level of $\mathrm{SpoOA}^{*}$, the competence is again possible. But if the SpoOA* reaches high levels, the competence is again inhibited, in this case by the Sinl-SinR mechanism (Fujita et al., 2005). In contrast, ComK can also regulate sporulation during competence. ComK activates the expression of RapH, a part of the Rap system, which dephosphorylates again SpoOF, a phosphotransferase of the sporulation initiation phosphorelay (Smits et al., 2007). This might also be the reason for the observation, that high comKS levels have a negative effect on the sporulation 
of the citZ-icd-mdh mutant. The lack of peptidoglycan synthesis cannot be the reason for the reduced spore formation in this case, since the sugar and carbon source mannitol is added to induce competence.

To conclude, the TCA cycle enzymes are not only important for central carbon metabolism, furthermore they also provide links to genetic competence, the cell morphology and sporulation. The blueprint of a minimal cell does not include the TCA cycle, since in the complex LB-glc medium, enough glucose is provided to gain energy from glycolysis and pentose-phosphate pathway (Reuß et al., 2016). Furthermore, the genes of the TCA cycle and also the respiratory chain are repressed in the presence of glucose, whereas the genes of glycolysis and overflow metabolism are upregulated (Blencke et al., 2003).. Although, the current MiniBacillus strain is already unable to form spores, competence is needed to sustain a stable working strain. Furthermore, the cell morphology can be critical, since this might have further impact on other cellular processes and the stability of the strain.

\subsection{The serine/ threonine transporter of $B$. subtilis}

High concentrations of serine in minimal medium have a growth inhibitory effect on $B$. subtilis cells (Lachowicz et al., 1996). This indicates a potential complex regulation of serine uptake to sustain a non-toxic level of serine in the cell. Similarly, serine inhibits also the growth of $E$. coli cells in high concentrations (Hama et al., 1990). Several serine uptake systems are described in E. coli, namely the serine-threonine system ( $\mathrm{SstT}, \mathrm{TdcC}$ ), the osmotic shock sensitive alanine, serine, threonine and leucine system (CycA) and a high-affintiy import system, specific for L-serine ( $\mathrm{SdaC}$ ) (Robbins and Oxender, 1973; Hama et al., 1988; Shao et al., 1994; Ogawa et al., 1998). This suggest that B. subtilis also harbours several serine uptake systems.

The results of this work indicate that the genes $y b e C, y b x G$ and $b c a P$ encode for serine/ threonine importers. YbeC seems to be a low-affinity serine transporter, which transports the major part of serine into cell. The $y b e C$ deletion strain has a clear growth advantage on C-glc minimal plates with a serine concentration that inhibits the growth of the wild type cells. The low-affinity transporters for the uptake of valine and isoleucine are not characterized, but they are only expressed and active under high substrate conditions (Belitsky, 2015). Although there was no change in expression of $\mathrm{YbeC}$ in the absence of serine, the expression needs to be further investigated under different serine concentrations to characterize YbeC further. Many suppressor mutants can be isolated that mutated $\mathrm{YbeC}$ in a way, that destroys the protein or remove the Cterminal part. Especially the mutation in the C-terminus of the protein is very interesting, since the C-terminus is most likely located in the cytoplasma and could harbour a regulatory function. It could sense a signal from the cell and adapt the import of certain intermediates. In contrast, the C- 
terminus could also transmit a signal to a regulatory mechanism in the cell. The L- and D-serine importer YhaO of enterohaemorrhagic E. coli (EHEC), is involved in the activation of a type II secretion system, which is essential for virulence (Connolly et al., 2016; Pifer et al., 2018). This suggests, that YbeC could also have a second regulatory function in the cell. The expression of the YbeC protein without the C-terminal part in E. coli shows, that it has not the same activity as the complete enzyme, since the deletion of the C-terminal part could also lead to a conformational change of the complete protein and thereby to an inactive protein.

Previous studies suggested the protein SteT to be a serine/ threonine of from the L-amino acid transporter (LAT) family. The transport activity was tested by the construction of proteoliposomes with the desired transporter (Bartoccioni et al., 2010; Rodríguez-Banqueri et al., 2016). However, this protein showed no serine import activity under the tested conditions in this work. But steT could be important under different conditions or since it is annotated as a serine/ threonine exchanger, it could also export serine out of the cell.

The transporters $\mathrm{YbxG}$ and $\mathrm{BcaP}$ were identified to be also involved in the serine import in this work. The triple deletion mutant of $y b e c, y b x G$ and $b c a P$ is resistant to even very high concentrations of $100 \mathrm{mM}$ serine. Interestingly, the single deletion of $b c a P$ and $y b x G$ leads not to the same growth advantage as the deletion of $y b e C$. Nevertheless, BcaP seems to have a greater impact than YbxG. Both proteins could be active as high-affinity transporters, or they are just minor low-affinity serine uptake systems. This needs to be further analyzed by testing the growth of the deletion strains in lower serine concentrations. Especially, the serine auxotrophic mutants need to be tested, since the serine uptake is here essential. Furthermore, the expression of the transporters at different serine and also threonine concentrations could be interesting. The expression of YbeC, $\mathrm{BcaP}$ and $\mathrm{YbxG}$ in E. coli shows almost the same growth inhibition. This could lead to the assumption that the transporters are differently regulated. Similarly, the valine and isoleucine transporter BrnQ has just a minor high-affinity transport activity, since its expression is lower than the expression of BcaP (Belitsky, 2015).

Not all serine importers of $B$. subtilis could be identified in this study, since the combination with the serA deletion is still possible. The serine auxotrophic strain with the deletion in the three identified serine transporters is still able to grow on plates with serine. This indicates, that serine can still be imported into the cell and that the transport is sufficient to sustain good growth and no excess of serine is imported to the cell that could be harmful. In the yeast like fungus Pneumocystis carinii, leucine, serine and glutamine are imported by the same transporter. But the transport is not dependent on sodium or the energy from the ATP hydrolysis and it is suspected to be a diffusion mechanism (Basselin et al., 2001). This could also be possible for the detected rest import by the 
deletion mutant of the three serine transporters and serA. Serine could diffuse into the cell by a channel protein, that is not energy dependent.

Furthermore, all three transporters have additionally a threonine import function. The two amino acids serine and threonine have both polar side chains and are very similar to each other (Figure 53).

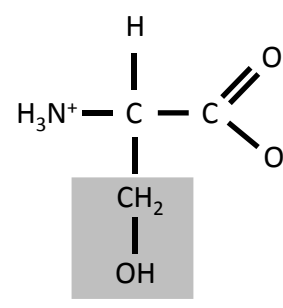

Serine

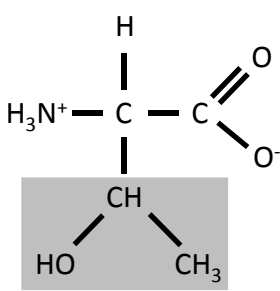

Threonine

Figure 53: The amino acids serine and threonine are very similar. Both molecules harbour a polar side chain.

Some serine/ threonine importers were already described in other organisms. The protein SstT from E. coli, is a $\mathrm{Na}+$ / serine importer with an additional threonine import function (Ogawa et al., 1998). Furthermore, a similar protein SstT from Porphyromonas gingivalis is known (Dashper et al., 2001). The threonine import activities of the three serine/threonine importers $\mathrm{YbeC}, \mathrm{BcaP}$ and $\mathrm{YbxG}$ of $B$. subtilis show also differences. Since high threonine concentrations were shown to be toxic for the cell, this effect was used in this work to test if the deletion of one transporter might lead to a growth advantage (Teas, 1950). Under the tested conditions, the deletion of bcaP led to the highest growth. This leads to the assumption, that $\mathrm{BcaP}$ is the low-affinity transporter for threonine. The deletion of $y b x G$ and $y b e C$ have just small effects and can possibly be considered as high-affinity transporter, but this needs to be further analysed. BcaP mediates additionally the high-affinity uptake of isoleucine and valine as a permease and it could be already shown that it is involved in the uptake of threonine (Belitsky, 2015). YbxG is a putative threonine transporter, since the deletion mutant shows resistance to the toxic threonine derivative 4-hydroxy-threonine (Commichau et al., 2015). The three proteins $\mathrm{YbeC}, \mathrm{YbxG}$ and BcaP are not the only threonine transporter, encoded in the genome of $B$. subtilis, since the combination with the threonine auxotrophic strain, the thrC deletion, is still possible. ThrC is the threonine synthase, that catalyses the last step in the threonine biosynthesis pathway from O-phospho-L-homoserine to threonine (Parsot, 1986). Interestingly, the triple importer deletion mutant with the thrC deletion cannot grow on very low concentrations of threonine. This might indicate, that all high-affinity threonine transporters are deleted, that would be active under low threonine conditions. If $y b e C$ is still in the strain ( $\Delta t h r C \Delta b c a P \Delta y b x G$ ), almost no difference to the deletion mutant of all three transporters can be observed. In contrast, if $y b x G$ remains in the strain ( $\Delta t h r C \Delta b c a P \Delta y b e C$ ), the strain growth better than the triple transporter 
deletion strain. These results suggest, that $\mathrm{YbxG}$ might be important at low threonine concentrations and could be therefore a high-affinity transporter.

Most amino acids transporters are able to transport several amino acids. It was previously reported that the branched-chain amino acid permease BcaP from Lactococcus lactis seems to additionally transport methionine into the cell (Den Hengst et al., 2006). The B. subtilis BcaP protein imports less isoleucine into the cell in the presence of the amino acids valine, serine, threonine, alanine and asparagine (Belitsky, 2015). A possible co-transport with serine was also tested in this work. The growth inhibitory effect of serine was used to identify amino acids that can compensate the toxicity. The amino acids threonine, asparagine, aspartate, glutamate, glutamine, arginine and proline were found to compensate the toxic effect of serine. Additionally, tyrosine, alanine and leucine could weakly compensate the effect. However, the compensation can be due to the import competition with another amino acid or due to the compensation of the toxic effect directly within the cell. Since alanine and asparagine were also found to reduce the isoleucine import function of BcaP, this transporter most likely imports these amino acids. The other identified amino acids are candidates for the co-transport by $\mathrm{YbeC}, \mathrm{YbxG}$ or the unidentified serine or threonine importer. This can only be elucidated, if all serine and threonine transporters are known. Strains with only one serine/ threonine transporter can then be tested for the reduced toxicity of serine or threonine in the presence of the identified amino acids. Especially the combination with the data from the the threonine toxicity compensation experiment might be interesting. Amino acids that are found in both experiments with serine and threonine are most ikely co-transported into the cell. In contrast, those amino acids that can be only found in the serine or threonine competition experiment are counteracting the toxicity in the cell.

The results of this work indicate, that $B$. subtilis harbours at least three serine/ threonine importers. The reason for that might be that the uptake of the different co-transported amino acids needs to be adapted to the environmental conditions. The expression of the transporters can therefore be differentially regulated. BcaP expression is under control of the global regulator CodY and it is repressed under high branched-chain amino acid concentrations (Molle et al., 2003). The regulation of $\mathrm{YbeC}$ is independent on the presence of serine in the cell (chapter 3.3.1.). Furthermore, the regulation of $y b x G$ and $y b e c$ need to be further analysed.

In the future, the missing serine/ threonine transporters need to be identified and a first approach therefore would be the screening of paralogous proteins of BcaP, YbeC and YbxG (Table 17). Several of these paralogous proteins are of known function, like GabP, the gamma-amino butyric acid permease and minor proline permease (Zaprasis et al., 2014). Some proteins are also of unknown function, that still need to be characterized. Another candidate for a threonine 
importer might also be SteT, since no serine uptake function of this serine/ threonine exchanger could be shown in this work.

Table 17: Paralogous proteins of the known serine/ threonine importers

\begin{tabular}{|l|l|}
\hline Serine/ threonine importer & Paralogous proteins \\
\hline BcaP & MtrA \\
\hline YbxG & RocC, RocE, YbgF, YdgF, AapA, GabP, YtnA, HutM, YcbW \\
\hline YbeC & YveA \\
\hline
\end{tabular}

\subsection{The regulatory role of serine}

Some amino acids were described to inhibit the cell growth and sporulation in high concentrations and are therefore toxic for $B$. subtilis. Especially, threonine, valine and isoleucine have an inhibitory effect (Lamb and Bott, 1979b; Lamb and Bott, 1979a). These toxic effects were used to analyze the regulatory mechanisms of amino acids in the cell. The threonine inhibitory effect was described as a block in valine biosynthesis. The cells starved of valine and their growth is therefore inhibited (Lamb and Bott, 1979b; Lamb and Bott, 1979a).

Serine can also inhibit the growth of $B$. subtilis if it is present in minimal medium as the single amino acid (Lachowicz et al., 1996). Since the exact mechanism of toxicity is unknown, this work also analyzed the target of serine inhibition further. B. subtilis rapidly forms different suppressor mutants on minimal plates with serine. The first characterized mutations affect the import of serine, by the mutation of the previously characterized serine/ threonine importer ybec. Several serine importers are known, but $\mathrm{YbeC}$ seems to be the low-affinity transporter, that is active under the tested high serine conditions. Different mutations in ybec could be identified, that destroy the protein or remove the C-terminal part, that seems to be important for the active protein. The second type of mutations upregulated the serine degradation pathway to lower the intracellular serine concentration. Finally, the third type of mutations lead to an increase in the threonine/ isoleucine biosynthesis pathway. This leads to the assumption, that serine might have a function in the inhibition of the threonine pathway. Some amino acids, like aspartate and glutamate can compensate the toxic effect of serine (Lachowicz et al., 1996). Furthermore, threonine can compensate the toxic effect, but more interestingly, also homoserine was able to compensate it. Therefore, the point of inhibition must be in the threonine pathway upstream of homoserine. The enzyme that catalyzes the reaction of L-aspartate semi-aldehyde to homoserine is the homoserine dehydrogenase Hom (Parsot and Cohen, 1988). The homoserine dehydrogenase is the target of the serine toxicity in E. coli, so this enzyme could also be the serine target in B. subtilis (Hama et al., 1990; Hama et al., 1991). The Hom enzyme from B. subtilis is partially inhibited by methionine, 
isoleucine, threonine and casamino acids. However, the repression of CAA was higher, than the effect of any tested combination of aspartate derived amino acids (Yeggy and Stahly, 1980). This indicates, that there must be at least one additional amino acid that can inhibit the activity of the homoserine dehydrogenase. The results of this work indicate that the repressing amino acid could be serine.

The Hom protein harbors a C-terminal ACT-domain (Parsot and Cohen, 1988). ACT-domains are named after the enzymes in which they were discovered first: aspartate kinase-chorismate mutase-TyrA. These domains can be found in different proteins from bacteria, archaea and eukaryotes. Most of them are involved in amino acid related pathways and the binding of amino acids to the ACT-domain often regulate the protein activity (Aravind and Koonin, 1999). The first described crystal structure was the ACT-domain of the phosphoglycerate dehydrogenase (PGDH) from $E$. coli, which is responsible for the first step in the biosynthesis of serine from pyruvate. Lserine can bind to the ACT-domain of this protein and regulate its activity. However, the exact mechanism is unknown (Schuller et al., 1995). Although the function of many ACT-domains from other organisms like E. coli is known, it is not much known about the ACT-domains from B. subtilis proteins. The L-serine dehydratase SdaAB-AA from B. subtilis also contains an ACT-domain in the $\beta$ chain encoded by $S d a A B$. It shows similarities to the ACT-domain in the PGDH of E. coli and furthermore the serine binding motif is also similar ( $\mathrm{Xu}$ and Grant, 2013). The ACT-domain of the homoserine dehydrogenase from $B$. subtilis could also be bound by serine and the activity is therefore down regulated. To conclude, the reason for the toxicity of serine might be the binding of serine to the ACT-domain of the Hom protein and the resulting inhibition of the enzyme activity. This leads finally to a lack of threonine and the amino acids downstream of the pathway. In Corynebacterium glutamicum, the deletion of the C-terminus of the homoserine dehydrogenase leads to the inactivation of the threonine inhibitory effect on this protein (Archer et al., 1991). To analyse if the ACT-domain of the Hom enzyme is responsible for the toxic serine effect, a strain could be constructed, which harbours the Hom protein without the ACT-domain. This strain can be then tested on C-glc plates with high concentrations of serine. If the strain is resistant to serine and grows better than the wild type strain, serine might bind to the ACT-domain of the homoserine dehydrogenase and change thereby its activity. The modified Hom, without ACT-domain protein needs to be still active. The deletion of the C-terminus could also result in the inactivation of the enzyme. However, this experiment could prove that serine inhibits the threonine biosynthesis.

The fact that serine and threonine are imported by the same proteins also supports this thesis. In natural conditions, both amino acids are imported into the cell. Since threonine has also a regulatory function in the cell and is toxic in high concentrations, the amount of serine that should be similar, seems to regulate the threonine biosynthesis (Lamb and Bott, 1979b). Therefore, the 
amount of threonine does not increase by the biosynthesis and threonine cannot inhibit other pathways, like valine biosynthesis. But in C-glc minimal medium with serine, only serine is present and is transported into the cell. This leads to a decrease in threonine biosynthesis, which is essential under these conditions, since no threonine is available in the medium. Therefore, suppressor mutations accumulate in the uptake and degradation of serine or in the threonine pathway to upregulate it again.

Since isoleucine is produced out of threonine, the addition of isoleucine to the C-glc medium with serine could possibly also reverse the toxic effect, at least partially. This compensation could be shown for E. coli cells (Hama et al., 1990). However, this effect was not visible in B. subtilis during this work (chapter 3.3.3.). This might be due to the fact, that high levels of isoleucine activate the global repressor CodY, which further represses the expression of the hom operon (Kriel et al., 2014). The repression leads than again to a decrease of the threonine level and this is a disadvantage for the cell. Furthermore, isoleucine can inhibit the Hom enzyme activity in B. subtilis (Yeggy and Stahly, 1980). In contrast, the ilvA mutation was found to counteract the toxic serine effect partially (chapter 3.4.). This could be due to the production of an intermediate level of isoleucine in the cell that is not too high to activate CodY, but enough to compensate the toxic effect partially. Another possibility is that the IlvA enzyme has a second minor function that is able to counteract the threonine auxotrophy. Similarly, the enzyme ThrC, which is normally involved in the threonine pathway, can partially take over the function if IlvA (Rosenberg et al., 2016). This needs to be further investigated. An interesting experiment could also be to measure the intracellular amino acid concentrations of the wild type strain. This could be measured in minimal medium supplied with high concentrations of serine, threonine or isoleucine. This might indicate the changes of amino acid composition in the cell upon the excess of each amino acid.

In E. coli, several other effects of high intracellular serine concentration were observed. It could be shown, that peptidoglycan synthesis and cell division was inhibited (Zhang and Newman, 2008; Zhang et al., 2010). In an attempt to create a serine producing E. coli strain, which tolerates high amounts of serine, several point mutations occurred. Beside mutations in serine importers and the homoserine dehydrogenase, also mutations in serine exporters occurred (Mundhada et al., 2016; Mundhada et al., 2017). So far are no serine exporters have been described in B. subtilis. The toxic effect of serine could also have other effects on the $B$. subtilis cell, except of the inhibition of the threonine pathway. Serine is often found to be a regulatory intermediate in the cell. L-serine shows a regulatory influence in mammalian cancer cells, since it is involved in cell proliferation by modulating the flux of glycolytic intermediates (Ye et al., 2012; Newman and Maddocks, 2017). Furthermore, serine has an influence in the expression of respiratory genes in plants (Timm et al., 2013). 
To conclude, high concentrations of serine cause probably an inhibition of the homoserine dehydrogenase Hom of $B$. subtilis. This leads to a decrease of threonine in the cell. Serine acts therefore as a regulator of threonine biosynthesis, which is part of the cells complex regulatory mechanism to fit the needs of amino acids in the cell. This indicates an additional regulatory mechanism of amino acid homeostasis in the cell. The import of amino acids is tightly controlled by different transporter proteins, that are active under different amino acid concentrations or different lifestyles. Furthermore, the biosynthesis is often regulated by feedback inhibition of a biosynthetic enzyme by a produced amino acid. This works indicates a third level of regulation by amino acids that are not involved in the particular pathway. Furthermore, this adds an additional link of the import of an amino acid to its biosynthesis. Many genes for the biosynthesis of amino acids were shown to be higher expressed in the absence of casamino acids (Mäder et al., 2002). This might indicate that even more, similar regulatory mechanism can be found in $B$. subtilis. The biosynthesis pathways of all amino acids will be deleted in the MiniBacillus strain. Interestingly, the results of the toxicity of several amino acids indicate a highly regulated network of amino acid biosynthesis. The deletion of one amino acid biosynthesis pathway might have an effect on another pathway. Furthermore, it should be considered to not delete the degradation pathway of serine, before the biosynthesis of serine is deleted. This avoids the accumulation of serine in the cell. 


\section{References}

Aguilar Suárez, R., Stülke, J., and Dijl, J.M. van (2019) Less Is More: Toward a Genome-Reduced Bacillus Cell Factory for "Difficult Proteins". ACS Synth Biol 8: 99-108.

Alén, C., and Sonenshein, A.L. (1999) Bacillus subtilis aconitase is an RNA-binding protein. Proc Natl Acad Sci U S A 96: 10412-10417.

Allenby, N.E.E., O'Connor, N., Pragai, Z., Ward, A.C., Wipat, A., and Harwood, C.R. (2005) GenomeWide Transcriptional Analysis of the Phosphate Starvation Stimulon of Bacillus subtilis. J Bacteriol 187: 8063-8080.

Ara, K., Ozaki, K., Nakamura, K., Yamane, K., Sekiguchi, J., and Ogasawara, N. (2007) Bacillus minimum genome factory: effective utilization of microbial genome information. Biotechnol Appl Biochem 46: 169-178.

Aravind, L., and Koonin, E. V (1999) Gleaning non-trivial structural, functional and evolutionary information about proteins by iterative database searches. J Mol Biol 287: 1023-1040.

Archer, J.A., Solow-Cordero, D.E., and Sinskey, A.J. (1991) A C-terminal deletion in Corynebacterium glutamicum homoserine dehydrogenase abolishes allosteric inhibition by Lthreonine. Gene 107: 53-59.

Awasthi, D., Wang, L., Rhee, M.S., Wang, Q., Chauliac, D., Ingram, L.O., and Shanmugam, K.T. (2018) Metabolic engineering of Bacillus subtilis for production of D-lactic acid. Biotechnol Bioeng 115: 453-463.

Bartoccioni, P., Rio, C. Del, Ratera, M., Kowalczyk, L., Baldwin, J.M., Zorzano, A., et al. (2010) Role of transmembrane domain 8 in substrate selectivity and translocation of SteT, a member of the L-Amino acid Transporter (LAT) family. J Biol Chem 285: 28764-28776.

Basselin, M., Qiu, Y.H., Lipscomb, K.J., and Kaneshiro, E.S. (2001) Uptake of the neutral amino acids glutamine, leucine, and serine by Pneumocystis carinii. Arch Biochem Biophys 391: 90-98.

Baureder, M., and Hederstedt, L. (2011) Production, purification and detergent exchange of isotopically labeled Bacillus subtilis cytochrome b558 (SdhC). Protein Expr Purif 80: 97-101.

Belitsky, B.R. (2011) Indirect Repression by Bacillus subtilis CodY via Displacement of the Activator of the Proline Utilization Operon. J Mol Biol 413: 321-336.

Belitsky, B.R. (2015) Role of branched-chain amino acid transport in Bacillus subtilis CodY activity. J Bacteriol 197: 1330-1338.

Belitsky, B.R., Brinsmade, S.R., and Sonenshein, A.L. (2015) Intermediate Levels of Bacillus subtilis CodY Activity Are Required for Derepression of the Branched-Chain Amino Acid Permease, BraB. PLoS Genet 11: 1-22.

Belitsky, B.R., and Sonenshein, A.L. (1998) Role and regulation of Bacillus subtilis glutamate dehydrogenase genes. J Bacteriol 180: 6298-6305.

Bennett, B.D., Kimball, E.H., Gao, M., Osterhout, R., Dien, S.J. Van, and Rabinowitz, J.D. (2009) Absolute metabolite concentrations and implied enzyme active site occupancy in Escherichia coli. Nat Chem Biol 5: 593-599.

Bennett, G.M., and Moran, N.A. (2013) Small, smaller, smallest: the origins and evolution of ancient dual symbioses in a Phloem-feeding insect. Genome Biol Evol 5: 1675-1688.

Blencke, H.-M., Homuth, G., Ludwig, H., Mäder, U., Hecker, M., and Stülke, J. (2003) Transcriptional profiling of gene expression in response to glucose in Bacillus subtilis: regulation of the central metabolic pathways. Metab Eng 5: 133-149. 
Blötz, C., Commichau, F.M., and Stülke, J. (2017) Methods in Molecular Biology of Bacteria. 3rd ed., Georg-August-University Göttingen.

Borriss, R., Danchin, A., Harwood, C.R., Médigue, C., Rocha, E.P.C., Sekowska, A., and Vallenet, D. (2018) Bacillus subtilis, the model Gram-positive bacterium: 20 years of annotation refinement. Microb Biotechnol 11: 3-17.

Bosdriesz, E., Wortel, M.T., Haanstra, J.R., Wagner, M.J., la Torre Cortés, P. de, and Teusink, B. (2018) Low affinity uniporter carrier proteins can increase net substrate uptake rate by reducing efflux. Sci Rep 8: 5576.

Bouraoui, H., Ventroux, M., Noirot-Gros, M.F., Deutscher, J., and Joyet, P. (2013) Membrane sequestration by the EIIB domain of the mannitol permease MtIA activates the Bacillus subtilis $m t /$ operon regulator MtIR. Mol Microbiol 87: 789-801.

Bradford, M.M. (1976) A rapid and sensitive method for the quantitation of microgram quantities of protein utilizing the principle of protein-dye binding. Anal Biochem 72: 248-254.

Brock, M., Maerker, C., Schütz, A., Völker, U., and Buckel, W. (2002) Oxidation of propionate to pyruvate in Escherichia coli. Involvement of methylcitrate dehydratase and aconitase. Eur J Biochem 269: 6184-6194.

Bryan, E.M., Beall, B.W., and Moran, C.P. (1996) A sigma E dependent operon subject to catabolite repression during sporulation in Bacillus subtilis. J Bacteriol 178: 4778-4786.

Burbulys, D., Trach, K.A., and Hoch, J.A. (1991) Initiation of sporulation in B. subtilis is controlled by a multicomponent phosphorelay. Cell 64: 545-552.

Burgard, A.P., Vaidyaraman, S., and Maranas, C.D. (2001) Minimal reaction sets for Escherichia coli metabolism under different growth requirements and uptake environments. Biotechnol Prog 17: 791-797.

Carlsson, P., and Hederstedt, L. (1989) Genetic characterization of Bacillus subtilis odhA and odhB, encoding 2-oxoglutarate dehydrogenase and dihydrolipoamide transsuccinylase, respectively. J Bacteriol 171: 3667-3672.

Chen, S., Xu, X.L., and Grant, G.A. (2012) Allosteric activation and contrasting properties of L-serine dehydratase types 1 and 2. Biochemistry 51: 5320-5328.

Chi, B.K., Roberts, A.A., Huyen, T.T.T., Bäsell, K., Becher, D., Albrecht, D., et al. (2013) Sbacillithiolation protects conserved and essential proteins against hypochlorite stress in firmicutes bacteria. Antioxid Redox Signal 18: 1273-1295.

Commichau, F.M. (2006) Regulation of glutamate synthesis in Bacillus subtilis by the glutamate dehydrogenase RocG and the activator protein GltC.

Commichau, F.M., Alzinger, A., Sande, R., Bretzel, W., Reuß, D.R., Dormeyer, M., et al. (2015) Engineering Bacillus subtilis for the conversion of the antimetabolite 4-hydroxy-l-threonine to pyridoxine. Metab Eng 29: 196-207.

Commichau, F.M., Pietack, N., and Stülke, J. (2013) Essential genes in Bacillus subtilis: a reevaluation after ten years. Mol Biosyst 9: 1068-1075.

Commichau, F.M., and Stülke, J. (2008) Trigger enzymes: bifunctional proteins active in metabolism and in controlling gene expression. Mol Microbiol 67: 692-702.

Condon, C., Rourera, J., Brechemier-Baey, D., and Putzer, H. (2002) Ribonuclease M5 has few, if any, mRNA substrates in Bacillus subtilis. J Bacteriol 184: 2845-2849.

Connolly, J.P.R., Gabrielsen, M., Goldstone, R.J., Grinter, R., Wang, D., Cogdell, R.J., et al. (2016) A Highly Conserved Bacterial D-Serine Uptake System Links Host Metabolism and Virulence. PLoS Pathog 12: e1005359. 
Craig, J.E., Ford, M.J., Blaydon, D.C., and Sonenshein, A.L. (1997) A null mutation in the Bacillus subtilis aconitase gene causes a block in Spo0A-phosphate-dependent gene expression. J Bacteriol 179: 7351-7359.

Dajnowicz, S., Parks, J.M., Hu, X., Gesler, K., Kovalevsky, A.Y., and Mueser, T.C. (2017) Direct evidence that an extended hydrogen-bonding network influences activation of pyridoxal $5^{\prime}$ phosphate in aspartate aminotransferase. J Biol Chem 292: 5970-5980.

Daniel, R.A., and Errington, J. (1993) DNA sequence of the murE-murD region of Bacillus subtilis 168. J Gen Microbiol 139: 361-370.

Dashper, S.G., Brownfield, L., Slakeski, N., Zilm, P.S., Rogers, A.H., and Reynolds, E.C. (2001) Sodium ion-driven serine/threonine transport in Porphyromonas gingivalis. $J$ Bacteriol 183: 4142-4148.

Davidson, A.L., Dassa, E., Orelle, C., and Chen, J. (2008) Structure, Function, and Evolution of Bacterial ATP-Binding Cassette Systems. Microbiol Mol Biol Rev 72: 317-364.

Diethmaier, C., Pietack, N., Gunka, K., Wrede, C., Lehnik-Habrink, M., Herzberg, C., et al. (2011) A novel factor controlling bistability in Bacillus subtilis: the YmdB protein affects flagellin expression and biofilm formation. J Bacteriol 193: 5997-6007.

Driscoll, J.R., and Taber, H.W. (1992) Sequence organization and regulation of the Bacillus subtilis menBE operon. J Bacteriol 174: 5063-5071.

Dümmler, A., Lawrence, A.-M., and Marco, A. de (2005) Simplified screening for the detection of soluble fusion constructs expressed in E. coli using a modular set of vectors. Microb Cell Fact 4: 34.

Eder, S., Shi, L., Jensen, K., Yamane, K., and Hulett, F.M. (1996) A Bacillus subtilis secreted phosphodiesterase/alkaline phosphatase is the product of a Pho regulon gene, phoD. Microbiology 142: 2041-2047.

Eisenreich, W., Slaghuis, J., Laupitz, R., Bussemer, J., Stritzker, J., Schwarz, C., et al. (2006) 13 C isotopologue perturbation studies of Listeria monocytogenes carbon metabolism and its modulation by the virulence regulator PrfA. Proc Natl Acad Sci 103: 2040-2045.

Ellman, G.L. (1959) Tissue sulfhydryl groups. Arch Biochem Biophys 82: 70-77.

Fisher, S.H. (1999) Regulation of nitrogen metabolism in Bacillus subtilis: vive la différence! Mol Microbiol 32: 223-232.

Fraser, C.M., Gocayne, J.D., White, O., Adams, M.D., Clayton, R.A., Fleischmann, R.D., et al. (1995) The minimal gene complement of Mycoplasma genitalium. Science 270: 397-403.

Fujita, M., Gonzalez-Pastor, J.E., and Losick, R. (2005) High- and Low-Threshold Genes in the SpoOA Regulon of Bacillus subtilis. J Bacteriol 187: 1357-1368.

Fujita, Y. (2009) Carbon Catabolite Control of the Metabolic Network in Bacillus subtilis. Biosci Biotechnol Biochem 73: 245-259.

Gao, H., Jiang, X., Pogliano, K., and Aronson, A.I. (2002) The E1beta and E2 subunits of the Bacillus subtilis pyruvate dehydrogenase complex are involved in regulation of sporulation. J Bacteriol 184: $2780-2788$.

Gerwig, J., Kiley, T.B., Gunka, K., Stanley-Wall, N., and Stülke, J. (2014) The protein tyrosine kinases EpsB and PtkA differentially affect biofilm formation in Bacillus subtilis. Microbiology 160: 682691.

Gibson, D.G., Benders, G.A., Andrews-Pfannkoch, C., Denisova, E.A., Baden-Tillson, H., Zaveri, J., et al. (2008) Complete Chemical Synthesis, Assembly, and Cloning of a Mycoplasma genitalium Genome. Science (80-) 319: 1215-1220. 
Glaser, P., Frangeul, L., Buchrieser, C., Rusniok, C., Amend, A., Baquero, F., et al. (2001) Comparative genomics of Listeria species. Science 294: 849-852.

Gonzy-Tréboul, G., Zagorec, M., Rain-Guion, M.C., and Steinmetz, M. (1989) Phosphoenolpyruvate:sugar phosphotransferase system of Bacillus subtilis: nucleotide sequence of $p t s \mathrm{X}, \mathrm{pts} \mathrm{H}$ and the $5^{\prime}$-end of $p t s /$ and evidence for a ptsHI operon. Mol Microbiol 3: $103-112$.

Graves, L.M., and Switzer, R.L. (1990) Aspartokinase III, a new isozyme in Bacillus subtilis 168. J Bacteriol 172: 218-223.

Guérout-Fleury, A.M., Shazand, K., Frandsen, N., and Stragier, P. (1995) Antibiotic-resistance cassettes for Bacillus subtilis. Gene 167: 335-336.

Gundlach, J. (2017) Cyclic di-AMP signaling in Bacillus subtilis.

Gunka, K. (2011) The impact of the glutamate dehydrogenases on the link between carbon and nitrogen metabolism in Bacillus subtilis.

Halbedel, S., Hames, C., and Stülke, J. (2007) Regulation of carbon metabolism in the mollicutes and its relation to virulence. J Mol Microbiol Biotechnol 12: 147-154.

Hama, H., Kayahara, T., Tsuda, M., and Tsuchiya, T. (1991) Inhibition of homoserine dehydrogenase I by L-serine in Escherichia coli. J Biochem 109: 604-608.

Hama, H., Shimamoto, T., Tsuda, M., and Tsuchiya, T. (1987) Properties of a Na+-coupled serinethreonine transport system in Escherichia coli. Biochim Biophys Acta 905: 231-239.

Hama, H., Shimamoto, T., Tsuda, M., and Tsuchiya, T. (1988) Characterization of a novel L-serine transport system in Escherichia coli. J Bacteriol 170: 2236-2239.

Hama, H., Sumita, Y., Kakutani, Y., Tsuda, M., and Tsuchiya, T. (1990) Target of serine inhibition in Escherichia coli. Biochem Biophys Res Commun 168: 1211-1216.

Hammerschlag, M.R. (2001) Mycoplasma pneumoniae infections. Curr Opin Infect Dis 14: 181-186.

Hanington, P.C., Barreda, D.R., and Belosevic, M. (2006) A novel hematopoietic granulin induces proliferation of goldfish (Carassius auratus L.) macrophages. J Biol Chem 281: 9963-9970.

Hanson, R.S., and Cox, D.P. (1967) Effect of different nutritional conditions on the synthesis of tricarboxylic acid cycle enzymes. J Bacteriol 93: 1777-1787.

Hao, T., Han, B., Ma, H., Fu, J., Wang, H., Wang, Z., et al. (2013) In silico metabolic engineering of Bacillus subtilis for improved production of riboflavin, Egl-237, (R,R)-2,3-butanediol and isobutanol. Mol Biosyst 9: 2034-2044.

Hashimoto, M., Ichimura, T., Mizoguchi, H., Tanaka, K., Fujimitsu, K., Keyamura, K., et al. (2005) Cell size and nucleoid organization of engineered Escherichia coli cells with a reduced genome. Mol Microbiol 55: 137-149.

Hederstedt, L., and Rutberg, L. (1981) Succinate dehydrogenase--a comparative review. Microbiol Rev 45: 542-555.

Hederstedt, L., and Rutberg, L. (1983) Orientation of succinate dehydrogenase and cytochrome b558 in the Bacillus subtilis cytoplasmic membrane. J Bacteriol 153: 57-65.

Hengst, C.D. Den, Groeneveld, M., Kuipers, O.P., and Kok, J. (2006) Identification and Functional Characterization of the Lactococcus lactis Acid Permease BcaP ( CtrA ). J Bacteriol 188: 32803289.

Henke, S.K., and Cronan, J.E. (2014) Successful conversion of the Bacillus subtilis BirA Group II biotin protein ligase into a Group I ligase. PLoS One 9: e96757. 
Herzberg, C., Weidinger, L.A.F., Dörrbecker, B., Hübner, S., Stülke, J., and Commichau, F.M. (2007) SPINE: a method for the rapid detection and analysis of protein-protein interactions in vivo. Proteomics 7: 4032-4035.

Higgins, C.F., Hiles, I.D., Salmond, G.P., Gill, D.R., Downie, J.A., Evans, I.J., et al. (1986) A family of related ATP-binding subunits coupled to many distinct biological processes in bacteria. Nature 323: $448-450$.

Himmelreich, R., Hilbert, H., Plagens, H., Pirkl, E., Li, B.C., and Herrmann, R. (1996) Complete sequence analysis of the genome of the bacterium Mycoplasma pneumoniae. Nucleic Acids Res 24: 4420-4449.

Hirokawa, Y., Kawano, H., Tanaka-Masuda, K., Nakamura, N., Nakagawa, A., Ito, M., et al. (2013) Genetic manipulations restored the growth fitness of reduced-genome Escherichia coli. J Biosci Bioeng 116: 52-58.

Hoch, J.A., and Nester, E.W. (1973) Gene-enzyme relationships of aromatic acid biosynthesis in Bacillus subtilis. J Bacteriol 116: 59-66.

Holberger, L.E., Garza-Sánchez, F., Lamoureux, J., Low, D.A., and Hayes, C.S. (2012) A novel family of toxin/antitoxin proteins in Bacillus species. FEBS Lett 586: 132-136.

Hullo, M.-F., Auger, S., Soutourina, O., Barzu, O., Yvon, M., Danchin, A., and Martin-Verstraete, I. (2007) Conversion of methionine to cysteine in Bacillus subtilis and its regulation. J Bacteriol 189: 187-197.

Hutchison, C.A., Chuang, R.-Y., Noskov, V.N., Assad-Garcia, N., Deerinck, T.J., Ellisman, M.H., et al. (2016) Design and synthesis of a minimal bacterial genome. Science 351: aad6253.

Ishii, Y., Matsuura, Y., Kakizawa, S., Nikoh, N., and Fukatsu, T. (2013) Diversity of bacterial endosymbionts associated with Macrosteles leafhoppers vectoring phytopathogenic phytoplasmas. Appl Environ Microbiol 79: 5013-5022.

Jack, D.L., Paulsen, I.T., and Saier, J. (2000) The amino acid/polyamine/organocation (APC) superfamily of transporters specific for amino acids, polyamines and organocations. Microbiology 146: 1797-1814.

Jin, S., Jesús-Berríos, M. De, and Sonenshein, A.L. (1996) A Bacillus subtilis malate dehydrogenase gene. J Bacteriol 178: 560-563.

Jin, S., Levin, P.A., Matsuno, K., Grossman, A.D., and Sonenshein, A.L. (1997) Deletion of the Bacillus subtilis isocitrate dehydrogenase gene causes a block at stage I of sporulation. J Bacteriol 179: 4725-4732.

Jin, S., and Sonenshein, A.L. (1994a) Transcriptional Regulation of Bacillus subtilis Citrate Synthase Genes. J Bacteriol 176: 4680-4690.

Jin, S., and Sonenshein, A.L. (1994b) Identification of two distinct Bacillus subtilis citrate synthase genes. J Bacteriol 176: 4669-4679.

Jin, S., and Sonenshein, A.L. (1996) Characterization of the major citrate synthase of Bacillus subtilis. J Bacteriol 178: 3658-3660.

Johansson, C.J., and Pettersson, G. (1974) Kinetics of the inhibition of citrate synthase from pig heart by substrate analogues. Eur J Biochem 46: 5-11.

Jourlin-Castelli, C., Mani, N., Nakano, M.M., and Sonenshein, A.L. (2000) CcpC, a novel regulator of the LysR family required for glucose repression of the citB gene in Bacillus subtilis. J Mol Biol 295: 865-878.

Juhas, M., Eberl, L., and Glass, J.I. (2011) Essence of life: essential genes of minimal genomes. Trends Cell Biol 21: 562-568. 
Juhas, M., Reuss, D.R., Zhu, B., and Commichau, F.M. (2014) Bacillus subtilis and Escherichia coli essential genes and minimal cell factories after one decade of genome engineering. Microbiology 160: 2341-2351.

Jung, T., and Mack, M. (2018) Interaction of enzymes of the tricarboxylic acid cycle in Bacillus subtilis and Escherichia coli: a comparative study. FEMS Microbiol Lett 365.

Kawai, Y., Mercier, R., Wu, L.J., Domínguez-Cuevas, P., Oshima, T., and Errington, J. (2015) Cell Growth of Wall-Free L-Form Bacteria Is Limited by Oxidative Damage. Curr Bio/ 25: 1613-1618.

Kim, H.-J., Kim, S.-I., Ratnayake-Lecamwasam, M., Tachikawa, K., Sonenshein, A.L., and Strauch, M. (2003a) Complex regulation of the Bacillus subtilis aconitase gene. J Bacteriol 185: 16721680.

Kim, H.-J., Mittal, M., and Sonenshein, A.L. (2006) CcpC-dependent regulation of citB and Imo0847 in Listeria monocytogenes.J Bacteriol 188: 179-190.

Kim, H.J., Roux, A., and Sonenshein, A.L. (2002) Direct and indirect roles of CcpA in regulation of Bacillus subtilis Krebs cycle genes. Mol Microbiol 45: 179-190.

Kim, S.I., Jourlin-Castelli, C., Wellington, S.R., and Sonenshein, A.L. (2003b) Mechanism of repression by Bacillus subtilis CcpC, a LysR family regulator. J Mol Biol 334: 609-624.

Kobashi, N., Nishiyama, M., and Yamane, H. (2001) Characterization of aspartate kinase III of Bacillus subtilis. Biosci Biotechnol Biochem 65: 1391-1394.

Kobayashi, K., Ehrlich, S.D., Albertini, A., Amati, G., Andersen, K.K., Arnaud, M., et al. (2003) Essential Bacillus subtilis genes. Proc Natl Acad Sci U S A 100: 4678-4683.

Koo, B.M., Kritikos, G., Farelli, J.D., Todor, H., Tong, K., Kimsey, H., et al. (2017) Construction and Analysis of Two Genome-Scale Deletion Libraries for Bacillus subtilis. Cell Syst 4: 291-305.e7.

Kriel, A., Brinsmade, S.R., Tse, J.L., Tehranchi, A.K., Bittner, A.N., Sonenshein, A.L., and Wang, J.D. (2014) GTP dysregulation in Bacillus subtilis cells lacking (p)ppGpp results in phenotypic amino acid auxotrophy and failure to adapt to nutrient downshift and regulate biosynthesis genes. J Bacteriol 196: 189-201.

Kumpfmüller, J., Kabisch, J., and Schweder, T. (2013) An optimized technique for rapid genome modifications of Bacillus subtilis. J Microbiol Methods 95: 350-352.

Kunst, F., Ogasawara, N., Moszer, I., Albertini, A.M., Alloni, G., Azevedo, V., et al. (1997) The complete genome sequence of the gram-positive bacterium Bacillus subtilis. Nature 390: 249256.

Lachowicz, T.M., Morzejko, E., Panek, E., and Piatkowski, J. (1996) Inhibitory Action of Serine on Growth of Bacteria of the Genus Bacillus on Mineral Synthetic Media. Folia Microbiol (Praha) 41: 21-25.

Laemmli, U.K. (1970) Cleavage of structural proteins during the assembly of the head of bacteriophage T4. Nature 227: 680-685.

Lamb, D.H., and Bott, K.F. (1979a) Inhibition of Bacillus subtilis growth and sporulation by threonine. J Bacteriol 137: 213-220.

Lamb, D.H., and Bott, K.F. (1979b) Threonine inhibition of growth of Bacillus subtilis: positive selection for isoleucine auxotrophy. J Gen Microbiol 111: 433-435.

Lartigue, C., Glass, J.I., Alperovich, N., Pieper, R., Parmar, P.P., Hutchison, C.A., et al. (2007) Genome transplantation in bacteria: changing one species to another. Science 317: 632-638. 
Liao, C.-H., Yao, L.-L., and Ye, B.-C. (2014) Three genes encoding citrate synthases in Saccharopolyspora erythraea are regulated by the global nutrient-sensing regulators $G \ln R$, DasR, and CRP. Mol Microbiol 94: 1065-1084.

Lineweaver, H., and Burk, D. (1934) The Determination of Enzyme Dissociation Constants. J Am Chem Soc 56: 658-666.

Liu, W., Eder, S., and Hulett, F.M. (1998) Analysis of Bacillus subtilis tagAB and tagDEF expression during phosphate starvation identifies a repressor role for PhoP P. J Bacteriol 180: 753-758.

Lowe, P.N., Hodgson, J.A., and Perham, R.N. (1983) Dual role of a single multienzyme complex in the oxidative decarboxylation of pyruvate and branched-chain 2-oxo acids in Bacillus subtilis. Biochem J 215: 133-140.

Ludwig, H., Homuth, G., Schmalisch, M., Dyka, F.M., Hecker, M., and Stülke, J. (2001) Transcription of glycolytic genes and operons in Bacillus subtilis: evidence for the presence of multiple levels of control of the gapA operon. Mol Microbiol 41: 409-422.

Luttinger, A., Hahn, J., and Dubnau, D. (1996) Polynucleotide phosphorylase is necessary for competence development in Bacillus subtilis. Mol Microbiol 19: 343-356.

Maamar, H., and Dubnau, D. (2005) Bistability in the Bacillus subtilis K-state (competence) system requires a positive feedback loop. Mol Microbiol 56: 615-624.

Mäder, U., Hennig, S., Hecker, M., and Homuth, G. (2004) Transcriptional organization and posttranscriptional regulation of the Bacillus subtilis branched-chain amino acid biosynthesis genes. J Bacteriol 186: 2240-2252.

Mäder, U., Homuth, G., Scharf, C., Büttner, K., Bode, R., and Hecker, M. (2002) Transcriptome and proteome analysis of Bacillus subtilis gene expression modulated by amino acid availability. $J$ Bacteriol 184: 4288-4295.

Manolukas, J.T., Barile, M.F., Chandler, D.K., and Pollack, J.D. (1988) Presence of anaplerotic reactions and transamination, and the absence of the tricarboxylic acid cycle in mollicutes. $J$ Gen Microbiol 134: 791-800.

Martin-Verstraete, I., Débarbouillé, M., Klier, A., and Rapoport, G. (1992) Mutagenesis of the Bacillus subtilis ' $-12,-24$ ' promoter of the levanase operon and evidence for the existence of an upstream activating sequence. J Mol Biol 226: 85-99.

Matsson, M., Tolstoy, D., Aasa, R., and Hederstedt, L. (2000) The distal heme center in Bacillus subtilis succinate:quinone reductase is crucial for electron transfer to menaquinone. Biochemistry 39: 8617-8624.

Matsuno, K., Blais, T., Serio, A.W., Conway, T., Henkin, T.M., and Sonenshein, A.L. (1999) Metabolic imbalance and sporulation in an isocitrate dehydrogenase mutant of Bacillus subtilis. J Bacteriol 181: 3382-3391.

Melin, L., Magnusson, K., and Rutberg, L. (1987) Identification of the promoter of the Bacillus subtilis sdh operon. J Bacteriol 169: 3232-3236.

Mercier, R., Kawai, Y., and Errington, J. (2014) General principles for the formation and proliferation of a wall-free (L-form) state in bacteria. Elife 3: e04629.

Meyer, F.M., Gerwig, J., Hammer, E., Herzberg, C., Commichau, F.M., Völker, U., and Stülke, J. (2011) Physical interactions between tricarboxylic acid cycle enzymes in Bacillus subtilis: evidence for a metabolon. Metab Eng 13: 18-27.

Michna, R.H., Zhu, B., Mäder, U., and Stülke, J. (2016) SubtiWiki 2.0--an integrated database for the model organism Bacillus subtilis. Nucleic Acids Res 44: D654-662. 
Miles, J.S., and Guest, J.R. (1985) Complete nucleotide sequence of the fumarase gene (citG) of Bacillus subtilis 168. Nucleic Acids Res 13: 131-140.

Mirouze, N., Bidnenko, E., Noirot, P., and Auger, S. (2015) Genome-wide mapping of TnrA-binding sites provides new insights into the TnrA regulon in Bacillus subtilis. Microbiologyopen 4: 423435.

Mirouze, N., Desai, Y., Raj, A., and Dubnau, D. (2012) SpoOA P imposes a temporal gate for the bimodal expression of competence in Bacillus subtilis. PLoS Genet 8: e1002586.

Molle, V., Nakaura, Y., Shivers, R.P., Yamaguchi, H., Losick, R., Fujita, Y., and Sonenshein, A.L. (2003) Additional targets of the Bacillus subtilis global regulator CodY identified by chromatin immunoprecipitation and genome-wide transcript analysis. J Bacteriol 185: 1911-1922.

Morimoto, T., Kadoya, R., Endo, K., Tohata, M., Sawada, K., Liu, S., et al. (2008) Enhanced recombinant protein productivity by genome reduction in Bacillus subtilis. DNA Res 15: 73-81.

Moses, S., Sinner, T., Zaprasis, A., Stöveken, N., Hoffmann, T., Belitsky, B.R., et al. (2012) Proline utilization by Bacillus subtilis: uptake and catabolism. J Bacteriol 194: 745-758.

Mundhada, H., Schneider, K., Christensen, H.B., and Nielsen, A.T. (2016) Engineering of high yield production of L-serine in Escherichia coli. Biotechnol Bioeng 113: 807-816.

Mundhada, H., Seoane, J.M., Schneider, K., Koza, A., Christensen, H.B., Klein, T., et al. (2017) Increased production of L-serine in Escherichia coli through Adaptive Laboratory Evolution. Metab Eng 39: 141-150.

Nakamura, K., Ikeda, S., Matsuo, T., Hirata, A., Takehara, M., Hiyama, T., et al. (2011) Patch clamp analysis of the respiratory chain in Bacillus subtilis. Biochim Biophys Acta 1808: 1103-1107.

Nester, E.W., Jensen, R.A., and Nasser, D.S. (1969) Regulation of enzyme synthesis in the aromatic amino acid pathway of Bacillus subtilus. J Bacteriol 97: 83-90.

Newman, A.C., and Maddocks, O.D.K. (2017) Serine and Functional Metabolites in Cancer. Trends Cell Biol 27: 645-657.

Oesterle, S., Wuethrich, I., and Panke, S. (2017) Toward Genome-Based Metabolic Engineering in Bacteria. Adv Appl Microbiol 101: 49-82.

Ogawa, W., Kayahara, T., Tsuda, M., Mizushima, T., and Tsuchiya, T. (1997) Isolation and characterization of an Escherichia coli mutant lacking the major serine transporter, and cloning of a serine transporter gene. J Biochem 122: 1241-1245.

Ogawa, W., Kim, Y.M., Mizushima, T., and Tsuchiya, T. (1998) Cloning and expression of the gene for the Na+-coupled serine transporter from Escherichia coli and characteristics of the transporter. J Bacteriol 180: 6749-6752.

Oh, Y.-K., Palsson, B.O., Park, S.M., Schilling, C.H., and Mahadevan, R. (2007) Genome-scale reconstruction of metabolic network in Bacillus subtilis based on high-throughput phenotyping and gene essentiality data. J Biol Chem 282: 28791-28799.

Oliynyk, M., Samborskyy, M., Lester, J.B., Mironenko, T., Scott, N., Dickens, S., et al. (2007) Complete genome sequence of the erythromycin-producing bacterium Saccharopolyspora erythraea NRRL23338. Nat Biotechnol 25: 447-453.

Omasits, U., Ahrens, C.H., Müller, S., and Wollscheid, B. (2014) Protter: interactive protein feature visualization and integration with experimental proteomic data. Bioinformatics 30: 884-886.

Parsot, C. (1986) Evolution of biosynthetic pathways: a common ancestor for threonine synthase, threonine dehydratase and D-serine dehydratase. EMBO J 5: 3013-3019. 
Parsot, C., and Cohen, G.N. (1988) Cloning and nucleotide sequence of the Bacillus subtilis hom gene coding for homoserine dehydrogenase. Structural and evolutionary relationships with Escherichia coli aspartokinases-homoserine dehydrogenases I and II. J Biol Chem 263: 1465414660.

Pechter, K.B., Meyer, F.M., Serio, A.W., Stülke, J., and Sonenshein, A.L. (2013) Two roles for aconitase in the regulation of tricarboxylic acid branch gene expression in Bacillus subtilis. J Bacteriol 195: 1525-1537.

Pifer, R., Russell, R.M., Kumar, A., Curtis, M.M., and Sperandio, V. (2018) Redox, amino acid, and fatty acid metabolism intersect with bacterial virulence in the gut. Proc Natl Acad Sci U S A 115: E10712-E10719.

Piggot, P.J., and Hilbert, D.W. (2004) Sporulation of Bacillus subtilis. Curr Opin Microbiol 7: 579586.

Pohl, S., Bhavsar, G., Hulme, J., Bloor, A.E., Misirli, G., Leckenby, M.W., et al. (2013) Proteomic analysis of Bacillus subtilis strains engineered for improved production of heterologous proteins. Proteomics 13: 3298-3308.

Ponce-de-Leon, M.M., and Pizer, L.I. (1972) Serine biosynthesis and its regulation in Bacillus subtilis. J Bacteriol 110: 895-904.

Postma, P.W., Lengeler, J.W., and Jacobson, G.R. (1993) Phosphoenolpyruvate:carbohydrate phosphotransferase systems of bacteria. Microbiol Rev 57: 543-594.

Prágai, Z., Allenby, N.E.E., O'Connor, N., Dubrac, S., Rapoport, G., Msadek, T., and Harwood, C.R. (2004) Transcriptional Regulation of the phoPR Operon in Bacillus subtilis. J Bacteriol 186: 1182-1190.

Qi, H., Li, B.-Z., Zhang, W.-Q., Liu, D., and Yuan, Y.-J. (2015) Modularization of genetic elements promotes synthetic metabolic engineering. Biotechnol Adv 33: 1412-1419.

Qin, X., and Taber, H.W. (1996) Transcriptional regulation of the Bacillus subtilis menp1 promoter. J Bacteriol 178: 705-713.

Quentin, Y., Fichant, G., and Denizot, F. (1999) Inventory, assembly and analysis of Bacillus subtilis $A B C$ transport systems. J Mol Biol 287: 467-484.

Rahmer, R., Morabbi Heravi, K., and Altenbuchner, J. (2015) Construction of a Super-Competent Bacillus subtilis 168 Using the P mt/A -comKS Inducible Cassette. Front Microbiol 6: 1431.

Ramaley, R.F., and Hudock, M.O. (1973) Purification and properties of isocitrate dehydrogenase (NADP) from Thermus aquaticus YT-1, Bacillus subtilis-168 and Chlamydomonas reinhardti-Y2. Biochim Biophys Acta 315: 22-36.

Reddick, J.J., Sirkisoon, S., Dahal, R.A., Hardesty, G., Hage, N.E., Booth, W.T., et al. (2017) First Biochemical Characterization of a Methylcitric Acid Cycle from Bacillus subtilis Strain 168. Biochemistry 56: 5698-5711.

Reddick, J.J., and Williams, J.K. (2008) The $m m g A$ gene from Bacillus subtilis encodes a degradative acetoacetyl-CoA thiolase. Biotechnol Lett 30: 1045-1050.

Reig, N., Rio, C. Del, Casagrande, F., Ratera, M., Gelpí, J.L., Torrents, D., et al. (2007) Functional and structural characterization of the first prokaryotic member of the L-amino acid transporter (LAT) family: A model for APC transporters. J Biol Chem 282: 13270-13281.

Reuß, D.R. (2017) Large-scale genome reduction in bacteria: From Bacillus subtilis to MiniBacillus.

Reuß, D.R., Altenbuchner, J., Mäder, U., Rath, H., Ischebeck, T., Sappa, P.K., et al. (2017) Largescale reduction of the Bacillus subtilis genome: consequences for the transcriptional network, resource allocation, and metabolism. Genome Res 27: 289-299. 
Reuß, D.R., Commichau, F.M., Gundlach, J., Zhu, B., and Stülke, J. (2016) The Blueprint of a Minimal Cell: MiniBacillus. Microbiol Mol Biol Rev 80: 955-987.

Robbins, J.C., and Oxender, D.L. (1973) Transport systems for alanine, serine, and glycine in Escherichia coli K 12. J Bacteriol 116: 12-18.

Rodionov, D.A., Vitreschak, A.G., Mironov, A.A., and Gelfand, M.S. (2003) Regulation of lysine biosynthesis and transport genes in bacteria: yet another RNA riboswitch? Nucleic Acids Res 31: 6748-6757.

Rodríguez-Banqueri, A., Errasti-Murugarren, E., Bartoccioni, P., Kowalczyk, L., Perálvarez-Marín, A., Palacín, M., and Vázquez-Ibar, J.L. (2016) Stabilization of a prokaryotic LAT transporter by random mutagenesis. J Gen Physiol 147: 353-368.

Rosales-Mendoza, S., and Angulo, C. (2015) Bacillus subtilis comes of age as a vaccine production host and delivery vehicle. Expert Rev Vaccines 14: 1135-1148.

Rosenberg, J., Müller, P., Lentes, S., Thiele, M.J., Zeigler, D.R., Tödter, D., et al. (2016) ThrR, a DNAbinding transcription factor involved in controlling threonine biosynthesis in Bacillus subtilis. Mol Microbiol 101: 879-893.

Saier, Jr, M.H. (2000) Families of transmembrane transporters selective for amino acids and their derivatives. Microbiology 146: 1775-1795.

Saier, M.H. (2000) A functional-phylogenetic classification system for transmembrane solute transporters. Microbiol Mol Biol Rev 64: 354-411.

Saier, M.H., and Reizer, J. (1992) Proposed uniform nomenclature for the proteins and protein domains of the bacterial phosphoenolpyruvate: sugar phosphotransferase system. J Bacteriol 174: $1433-1438$.

Saizieu, A. de, Vankan, P., Vockler, C., and Loon, A.P.G.M. van (1997) The trp RNA-binding attenuation protein (TRAP) regulates the steady-state levels of transcripts of the Bacillus subtilis folate operon. Microbiology 143: 979-989.

Sambrook, J., Fritsch, E.F., and Maniatis, T. (1989) Molecular cloning: a laboratory manual. 2nd ed., Cold Spring Harbor Laboratory Press, Cold Spring Harbor, New York.

Sanger, F., Nicklen, S., and Coulson, A.R. (1992) DNA sequencing with chain-terminating inhibitors. 1977. Biotechnology 24: 104-108.

Santana, M., Kunst, F., Hullo, M.F., Rapoport, G., Danchin, A., and Glaser, P. (1992) Molecular cloning, sequencing, and physiological characterization of the qox operon from Bacillus subtilis encoding the aa3-600 quinol oxidase. J Biol Chem 267: 10225-10231.

Sauer, U., Hatzimanikatis, V., Bailey, J.E., Hochuli, M., Szyperski, T., and Wüthrich, K. (1997) Metabolic fluxes in riboflavin-producing Bacillus subtilis. Nat Biotechnol 15: 448-452.

Saxild, H.H., Brunstedt, K., Nielsen, K.I., Jarmer, H., and Nygaard, P. (2001) Definition of the Bacillus subtilis PurR operator using genetic and bioinformatic tools and expansion of the PurR regulon with glyA, guaC, pbuG, xpt-pbuX, yqhZ-folD, and pbuO. J Bacteriol 183: 6175-6183.

Schilling, O., Herzberg, C., Hertrich, T., Vörsmann, H., Jessen, D., Hübner, S., et al. (2006) Keeping signals straight in transcription regulation: specificity determinants for the interaction of a family of conserved bacterial RNA-protein couples. Nucleic Acids Res 34: 6102-6115.

Schirmer, F., Ehrt, S., and Hillen, W. (1997) Expression, inducer spectrum, domain structure, and function of MopR, the regulator of phenol degradation in Acinetobacter calcoaceticus NCIB8250. J Bacteriol 179: 1329-1336.

Schuller, D.J., Grant, G.A., and Banaszak, L.J. (1995) The allosteric ligand site in the Vmax-type cooperative enzyme phosphoglycerate dehydrogenase. Nat Struct Biol 2: 69-76. 
Shao, Z., Lin, R.T., and Newman, E.B. (1994) Sequencing and characterization of the sdaC gene and identification of the sdaCB operon in Escherichia coli K12. Eur J Biochem 222: 901-907.

Singer, E., Silas, Y.B., Ben-Yehuda, S., and Pines, O. (2017) Bacterial fumarase and L-malic acid are evolutionary ancient components of the DNA damage response. Elife 6: e30927.

Smits, W.K., Bongiorni, C., Veening, J.-W., Hamoen, L.W., Kuipers, O.P., and Perego, M. (2007) Temporal separation of distinct differentiation pathways by a dual specificity Rap-Phr system in Bacillus subtilis. Mol Microbiol 65: 103-120.

Smits, W.K., Eschevins, C.C., Susanna, K.A., Bron, S., Kuipers, O.P., and Hamoen, L.W. (2005) Stripping Bacillus: ComK auto-stimulation is responsible for the bistable response in competence development. Mol Microbiol 56: 604-614.

Speck, E.L., and Freese, E. (1973) Control of metabolite secretion in Bacillus subtilis. J Gen Microbiol 78: 261-275.

Stephenson, K., and Harwood, C.R. (1998) Influence of a cell-wall-associated protease on production of alpha-amylase by Bacillus subtilis. Appl Environ Microbiol 64: 2875-2881.

Stülke, J., and Hillen, W. (2000) Regulation of carbon catabolism in Bacillus species. Annu Rev Microbiol 54: 849-880.

Sun, D.X., and Setlow, P. (1991) Cloning, nucleotide sequence, and expression of the Bacillus subtilis ans operon, which codes for L-asparaginase and L-aspartase. J Bacteriol 173: 38313845.

Teas, H.J. (1950) Mutants of Bacillus subtilis that require threonine or threonine plus methionine. J Bacteriol 59: 93-104.

Timm, S., Florian, A., Wittmiß, M., Jahnke, K., Hagemann, M., Fernie, A.R., and Bauwe, H. (2013) Serine acts as a metabolic signal for the transcriptional control of photorespiration-related genes in Arabidopsis. Plant Physiol 162: 379-389.

Tocheva, E.I., López-Garrido, J., Hughes, H.V., Fredlund, J., Kuru, E., Vannieuwenhze, M.S., et al. (2013) Peptidoglycan transformations during Bacillus subtilis sporulation. Mol Microbiol 88: 673-686.

Töwe, S., Leelakriangsak, M., Kobayashi, K., Duy, N. Van, Hecker, M., Zuber, P., and Antelmann, H. (2007) The MarR-type repressor MhqR (YkvE) regulates multiple dioxygenases/glyoxalases and an azoreductase which confer resistance to 2-methylhydroquinone and catechol in Bacillus subtilis. Mol Microbiol 66: 40-54.

Trachtenberg, S. (1998) Mollicutes-wall-less bacteria with internal cytoskeletons. J Struct Biol 124: 244-256.

Vandeyar, M.A., and Zahler, S.A. (1986) Chromosal insertions of Tn917 in Bacillus subtilis. J Bacteriol 167: 530-534.

Wacker, I., Ludwig, H., Reif, I., Blencke, H.-M., Detsch, C., and Stülke, J. (2003) The regulatory link between carbon and nitrogen metabolism in Bacillus subtilis: regulation of the gltAB operon by the catabolite control protein CcpA. Microbiology 149: 3001-3009.

Weinrauch, Y., Msadek, T., Kunst, F., and Dubnau, D. (1991) Sequence and properties of comQ, a new competence regulatory gene of Bacillus subtilis. J Bacteriol 173: 5685-5693.

Wenzel, M., and Altenbuchner, J. (2015) Development of a markerless gene deletion system for Bacillus subtilis based on the mannose phosphoenolpyruvate-dependent phosphotransferase system. Microbiology 161: 1942-1949.

Westers, H., Dorenbos, R., Dijl, J.M. van, Kabel, J., Flanagan, T., Devine, K.M., et al. (2003) Genome engineering reveals large dispensable regions in Bacillus subtilis. Mol Biol Evol 20: 2076-2090. 
Wolf, M., Müller, T., Dandekar, T., and Pollack, J.D. (2004) Phylogeny of Firmicutes with special reference to Mycoplasma (Mollicutes) as inferred from phosphoglycerate kinase amino acid sequence data. Int J Syst Evol Microbiol 54: 871-875.

Woodcock, D.M., Crowther, P.J., Doherty, J., Jefferson, S., DeCruz, E., Noyer-Weidner, M., et al. (1989) Quantitative evaluation of Escherichia coli host strains for tolerance to cytosine methylation in plasmid and phage recombinants. Nucleic Acids Res 17: 3469-3478.

Wörmann, M.E., Corrigan, R.M., Simpson, P.J., Matthews, S.J., and Gründling, A. (2011) Enzymatic activities and functional interdependencies of Bacillus subtilis lipoteichoic acid synthesis enzymes. Mol Microbiol 79: 566-583.

Wray, L. V., and Fisher, S.H. (1994) Analysis of Bacillus subtilis hut operon expression indicates that histidine-dependent induction is mediated primarily by transcriptional antitermination and that amino acid repression is mediated by two mechanisms: Regulation of transcription initiation . J Bacteriol 176: 5466-5473.

Wray, L. V, Ferson, A.E., Rohrer, K., and Fisher, S.H. (1996) TnrA, a transcription factor required for global nitrogen regulation in Bacillus subtilis. Proc Natl Acad Sci U S A 93: 8841-8845.

Xu, X.L., and Grant, G.A. (2013) Identification and characterization of two new types of bacterial Iserine dehydratases and assessment of the function of the ACT domain. Arch Biochem Biophys 540: 62-69.

Yan, X., Yu, H.J., Hong, Q., and Li, S.P. (2008) Cre/lox system and PCR-based genome engineering in Bacillus subtilis. Appl Environ Microbiol 74: 5556-5562.

Yang, M., Galizzi, A., and Henner, D. (1983) Nucleotide sequence of the amylase gene from Bacillus subtilis. Nucleic Acids Res 11: 237-249.

Yanisch-Perron, C., Vieira, J., and Messing, J. (1985) Improved M13 phage cloning vectors and host strains: nucleotide sequences of the M13mp18 and pUC19 vectors. Gene 33: 103-119.

Yao, L.-L., Liao, C.-H., Huang, G., Zhou, Y., Rigali, S., Zhang, B., and Ye, B.-C. (2014) GlnR-mediated regulation of nitrogen metabolism in the actinomycete Saccharopolyspora erythraea. Appl Microbiol Biotechnol 98: 7935-7948.

Ye, J., Mancuso, A., Tong, X., Ward, P.S., Fan, J., Rabinowitz, J.D., and Thompson, C.B. (2012) Pyruvate kinase $\mathrm{M} 2$ promotes de novo serine synthesis to sustain mTORC1 activity and cell proliferation. Proc Natl Acad Sci U S A 109: 6904-6909.

Yeggy, J.P., and Stahly, D.P. (1980) Sporulation and regulation of homoserine dehydrogenase in Bacillus subtilis. Can J Microbiol 26: 1386-1391.

Yoshida, K., Fujita, Y., and Ehrlich, S.D. (1999) Three asparagine synthetase genes of Bacillus subtilis. J Bacteriol 181: 6081-6091.

Yu, J., Ge, J., Heuveling, J., Schneider, E., and Yang, M. (2015) Structural basis for substrate specificity of an amino acid ABC transporter. Proc Natl Acad Sci U S A 112: 5243-5248.

Zaprasis, A., Hoffmann, T., Stannek, L., Gunka, K., Commichau, F.M., and Bremer, E. (2014) The Yaminobutyrate permease GabP serves as the third proline transporter of Bacillus subtilis. J Bacteriol 196: 515-526.

Zeigler, D.R., Prágai, Z., Rodriguez, S., Chevreux, B., Muffler, A., Albert, T., et al. (2008) The origins of 168, W23, and other Bacillus subtilis legacy strains. J Bacteriol 190: 6983-6995.

Zhang, X., El-Hajj, Z.W., and Newman, E. (2010) Deficiency in L-serine deaminase interferes with one-carbon metabolism and cell wall synthesis in Escherichia coli K-12. J Bacteriol 192: 55155525 . 
Zhang, X., and Newman, E. (2008) Deficiency in L-serine deaminase results in abnormal growth and cell division of Escherichia coli K-12. Mol Microbiol 69: 870-881.

Zhao, H., Roistacher, D.M., and Helmann, J.D. (2018) Aspartate deficiency limits peptidoglycan synthesis and sensitizes cells to antibiotics targeting cell wall synthesis in Bacillus subtilis. Mol Microbiol 109: 826-844.

Zschiedrich, C.P. (2014) Nebenwege des zentralen Kohlenstoffmetabolismus von Bacillus subtilis: Regulation der Methylglyoxalsynthase und der Zitratsynthase CitA. 


\section{Appendix}

\subsection{Materials}

\subsubsection{Chemicals}

Acetyl-CoA

Acrylamide

Agar

Agarose

Alanine

Ammonium iron (III) citrate

Ammonium Peroxydisulfate

Antibiotics

Arginine

Asparagine

Aspartate

B-Mercaptoethanol

Bacto agar

BSA

Bromphenol blue

$\mathrm{CaCl}_{2}$

CAA

CDP*

Coomassie Brilliant Blue, G250

Cysteine

Desthiobiotin

DMSO

dNTPs
Sigma-Aldrich, Munich

Roth, Karlsruhe

Roth, Karlsruhe

Peqlab, Erlangen

Roth, Karlsruhe

Sigma-Aldrich, Munich

Roth, Karlsruhe

Sigma-Aldrich, Munich

Roth, Karlsruhe

Sigma-Aldrich, Munich

Sigma-Aldrich, Munich

Merck, Darmstadt

Becton, Dickinson and Company, Heidelberg

Roth, Karlsruhe

Serva, Heidelberg

Sigma-Aldrich, Munich

Sigma-Aldrich, Munich

Roche Diagnostics, Mannheim

Roth, Karlsruhe

Sigma-Aldrich, Munich

IBA, Göttingen

Carl Roth, Karlsruhe

Roche Diagnostics, Mannheim 


\begin{tabular}{|c|c|}
\hline DTNB & Roth, Karlsruhe \\
\hline DTT & Roth, Karlsruhe \\
\hline Ethidium bromide & Roth, Karlsruhe \\
\hline $\mathrm{FeCl}_{3} \times 6 \mathrm{H}_{2} \mathrm{O}$ & Sigma-Aldrich, Munich \\
\hline $\begin{array}{l}\mathrm{FM}^{\mathrm{TM}} \text { 4-64 Dye (N-(3-Triethylammoniumpropyl)-4- } \\
\text { (6-(4-(Diethylamino) Phenyl) Hexatrienyl) } \\
\text { Pyridinium Dibromide) }\end{array}$ & ThermoFisher, Braunschweig \\
\hline D-Fructose-1,6-bisphosphate & Sigma-Aldrich, Munich \\
\hline D-Glucose & Merck, Darmstadt \\
\hline Glutamate & Sigma-Aldrich, Munich \\
\hline Glutamine & Roth, Karlsruhe \\
\hline Glycerine & Merck, Darmstadt \\
\hline Glycine & Sigma-Aldrich, Munich \\
\hline HDGreen ${ }^{\mathrm{TM}}$ plus & Intas, Göttingen \\
\hline Histidine & AppliChem, Darmstadt \\
\hline Homoserine & Sigma-Aldrich, Munich \\
\hline Imidazole & Sigma-Aldrich, Munich \\
\hline Isoleucine & Roth, Karlsruhe \\
\hline Isopropyl ß-D-1-thiogalactopyranoside & Peqlap, Erlangen \\
\hline $\mathrm{KCl}$ & Oxoid, Heidelberg \\
\hline $\mathrm{KHCO}_{3}$ & Roth, Karlsruhe \\
\hline Leucine & Sigma-Aldrich, Munich \\
\hline Lysine & Sigma-Aldrich, Munich \\
\hline Methionine & Sigma-Aldrich, Munich \\
\hline $\mathrm{MgCl}_{2}$ & Sigma-Aldrich, Munich \\
\hline $\mathrm{MgSO}_{4} \times 7 \mathrm{H}_{2} \mathrm{O}$ & Roth, Karlsruhe \\
\hline $\mathrm{MnCl}_{2} \times 4 \mathrm{H}_{2} \mathrm{O}$ & Roth, Karlsruhe \\
\hline $\mathrm{NADP}^{+}$ & Sigma-Aldrich, Munich \\
\hline
\end{tabular}




\begin{tabular}{|c|c|}
\hline $\mathrm{Ni}^{2+}$-nitrilotriacetic acid superflow & Qiagen, Hilden \\
\hline Nutrient Broth & Merck, Darmstadt \\
\hline ONPG & AppliChem, Darmstadt \\
\hline Oxaloacetate & Sigma-Aldrich, Munich \\
\hline Phenylalanine & Roth, Karlsruhe \\
\hline Proline & Sigma-Aldrich, Munich \\
\hline Serine & Sigma-Aldrich, Munich \\
\hline Skim milk powder, fat-free & Roth, Karlsruhe \\
\hline Sodium succinate & Fluka, Buchs, Switzerland \\
\hline Sodium Dodecyl Sulfate & Roth, Karlsruhe \\
\hline Strep-Tactin Sepharose & IBA, Göttingen \\
\hline Tetramethylethylenediamine (TEMED) & Roth, Karlsruhe \\
\hline Thiamine & Sigma-Aldrich, Munich \\
\hline Threonine & AppliChem, Darmstadt \\
\hline Tris(hydroxymethyl)aminomethane & Roth, Karlsruhe \\
\hline Trypton & Oxoid, Heidelberg \\
\hline Tween 20 & Sigma-Aldrich, Munich \\
\hline Tyrosine & Sigma-Aldrich, Munich \\
\hline Valine & Roth, Karlsruhe \\
\hline X-Gal & Peqlab, Erlangen \\
\hline Yeast extract & Oxoid, Hampshire, U.K. \\
\hline
\end{tabular}

Other chemicals were purchase from Merck, Serva, Sigma or Roth.

\subsubsection{Enzymes}

\begin{tabular}{ll}
\hline FastAP $^{\text {TM }}$ & ThermoFisher, Braunschweig \\
DreamTaq DNA polymerase & ThermoFisher, Braunschweig \\
Lysozym & Merck, Darmstadt \\
Phusion $^{\text {TM }}$ DNA polymerase & ThermoFisher, Braunschweig \\
\hline
\end{tabular}




\subsubsection{Materials}

\begin{tabular}{ll}
\hline 96-Well plates & Sarstedt, Nümbrecht \\
Centrifuge cups & Beckmann, München \\
Cuvettes (microliter, plastic) & Greiner, Nürtingen \\
Dialysis tube & Serva, Heidelberg \\
Falcon tubes (15 ml, $50 \mathrm{ml})$ & Sarstedt, Nümbrecht \\
Gene Amp Reaction Tubes (PCR) & Perkin Elmer, Weiterstadt \\
Glass pipettes & Brandt, Wertheim \\
Microlitre pipettes (2 $\mu \mathrm{l}, 20 \mu \mathrm{l}, 200 \mu \mathrm{l}, 1000 \mu \mathrm{l}$, & Eppendorf, Hamburg and Gilson, Düsseldorf \\
$5000 \mu l$ & \\
Petri dishes & Greiner, Nürtingen \\
Pipette tips & Sarstedt, Nümbrecht \\
Poly-Prep Chromatography Columns & Bio-Rad Laboratories GmbH, Munich \\
Polyvinylidene fluoride membrane (PVDF) & Bio-Rad Laboratories GmbH, Munich \\
Reaction tubes & Greiner, Nürtingen \\
Single-use syringes $(5 \mathrm{ml}, 10 \mathrm{ml})$ & Becton Dickson Drogheda, Ireland \\
\hline
\end{tabular}

\subsubsection{Instruments/ Equipment}

\begin{tabular}{ll}
\hline Autoclave & Zirbus technology, Bad Grund \\
Biofuge fresco & Heraeus Christ, Osterode \\
Fluorescence microscope Axioskop 40FL + & Zeiss, Göttingen \\
AxioCam MRm & \\
French pressure cell press & SLM Aminco, Lorch \\
GelDoc ${ }^{\mathrm{TM}}$ XR+ & Biorad, Munich \\
Gel electrophoresis apparatus & PeqLab, Erlangen
\end{tabular}




\begin{tabular}{ll}
\hline Ice machine & Ziegra, Isernhagen \\
Heating block Dri Block DB3 & Waasetec, Göttingen \\
Horizontal shaker 3006 & GFL, Burgwedel \\
LabCycler & SensorQuest, Göttingen \\
Mini-Protean III System & Bio-Rad, Munich \\
Nanodrop ND-1000 & ThermoFisher, Braunschweig \\
Open air shaker Innova 2300 & New Brunswick, Neu-Isenburg \\
pH meter Calimatic & Knick, Berlin \\
Microplate Reader SynergyMx & BioTek, Bad Friedrichshall \\
Refrigerated centrifuge & Kendro, Hanau \\
Scale & Sartorius, Göttingen \\
Special accuracy weighing machine & Sartorius, Göttingen \\
Spectral photometer & Amersham, Freiburg \\
Standard power pack & Bio-Rad, Munich \\
Thermocycler & Biometra, Göttingen \\
Ultra centrifuge, Sorvall Ultra Pro 80 & ThermoFisher, Braunschweig \\
Ultrasonic device & Millepore, Schwalbach \\
UV Transilluminator 2000 & \\
\hline & \\
Vortex & Bater desalination plant \\
\hline
\end{tabular}

\subsubsection{Commercial systems}

\begin{tabular}{ll}
\hline HDGreen DNA Stain & Intas, Göttingen \\
peqGOLD Bacterial DNA Kit & PeqLab, Erlangen \\
NucleoSpin Plasmid-Kit & Macherey-Nagel, Düren \\
Prestained Protein Marker (PageRuler) & ThermoFisher, Braunschweig \\
QIAquick PCR Purification Kit & Qiagen, Hilden \\
\hline
\end{tabular}




\subsubsection{Software and webpages}

\begin{tabular}{|c|c|c|}
\hline Program & Provider & Application \\
\hline AxioVision & Zeiss & Microscopy imaging \\
\hline ChemoStar Imager & Intas & Western Blot imaging \\
\hline Gen5 $^{\mathrm{TM}}$ Data Analysis Software & BioTek $^{\circledR}$ & Plate reader analysis \\
\hline Geneious 10.0.5 & Biomatters & DNA analysis \\
\hline ImageLab ${ }^{\mathrm{TM}}$ Software & BioRad & Geldoc imaging \\
\hline Mendeley Desktop & PDFTron $^{\text {TM }}$ Systems Inc. & Reference Manager \\
\hline Microsoft Office 365 & Microsoft Inc. & Data processing \\
\hline SubtiWiki 2.0 & Michna et al., 2016 & B. subtilis database \\
\hline Zen & Zeiss & Image processing \\
\hline
\end{tabular}

\subsection{Bacterial strains}

\subsubsection{B. subtilis strains constructed in this work}

\begin{tabular}{|c|c|c|}
\hline Strain & Genotype & Reference/ Construction \\
\hline GP2324 & $\begin{array}{l}\operatorname{trpC2} \text { ybec ( } \triangle \mathrm{bp} 340, \text { stop aa } 125) \\
\text { duplication (yokD [bp 43-819] -thyA [bp 510-795]) }\end{array}$ & $\begin{array}{l}\text { Suppressor mutant of wt on } \\
\text { high serine concentrations } \\
\text { (WGS) }\end{array}$ \\
\hline GP2325 & $\operatorname{trpC2}$ ybeC ( $\triangle$ bp 974, stop aa 350$)$ & $\begin{array}{l}\text { Suppressor mutant of wt on } \\
\text { high serine concentrations } \\
\text { (PCR) }\end{array}$ \\
\hline GP2326 & $\begin{array}{l}\operatorname{trpC2} \Delta \text { citRA::lox72 sacA::(phl-PxylA-cre) } \Delta \text { citZ-icd- } \\
\text { mdh::kan-lox }\end{array}$ & LFH-PCR $\rightarrow$ GP1755 \\
\hline GP2327 & $\begin{array}{l}\operatorname{trpC2} \Delta \text { citRA::lox72 sacA::(phl-PxylA-cre) } \Delta \text { citZ-icd- } \\
m d h:: l o x 72\end{array}$ & GP2326 + 1\% Xylose \\
\hline GP2328 & $\operatorname{trpC2}$ sacA::(phl-PxylA-cre) & pJK195 $\rightarrow 168$ \\
\hline GP2329 & $\begin{array}{l}\operatorname{trpC2} \Delta \text { citRA::lox72 sacA::(phl-PxylA-cre) } \Delta \text { citZ-icd- } \\
m d h:: l o x 72 \Delta \text { citB::kan-lox }\end{array}$ & LFH-PCR $\rightarrow$ GP2327 \\
\hline GP2330 & $\begin{array}{l}\operatorname{trpC2} \Delta \text { citRA::lox72 sacA::(phl-Pxy|A-cre) } \Delta \text { citZ-icd- } \\
m d h:: \text { lox72 } \Delta \text { citB::lox72 }\end{array}$ & GP2329 + 1\% Xylose \\
\hline
\end{tabular}




\begin{tabular}{|c|c|c|}
\hline GP2331 & $\operatorname{trpC2}$ sacA::(phl-PxylA-cre) $\Delta$ citZ-icd-mdh::kan-lox & LFH-PCR $\rightarrow$ GP2328 \\
\hline GP2332 & $\operatorname{trpC2}$ sacA::(phl-PxylA-cre) $\triangle$ odhAB::kan-lox & LFH-PCR $\rightarrow$ GP2328 \\
\hline GP2333 & $\operatorname{trpC2}$ sacA::(phl-Pxy|A-cre) $\Delta$ citZ-icd-mdh::lox72 & GP2331 + 1\% Xylose \\
\hline GP2334 & $\operatorname{trpC2}$ sacA::(phl-Pxy|A-cre) $\triangle$ odhAB::lox72 & GP2332 + 1\% Xylose \\
\hline GP2335 & $\begin{array}{l}\operatorname{trpC2} \Delta \text { citRA::lox72 sacA::(phl-PxylA-cre) } \Delta \text { citZ-icd- } \\
\text { mdh::lox72 } \Delta \text { citB::lox72 } \Delta \text { citG::lox72 } \Delta \text { sdhCAB::kan-lox }\end{array}$ & PCR GP2342 $\rightarrow$ GP2352 \\
\hline GP2336 & $\operatorname{trpC2}$ sacA::(phl-PxylA-cre) $\Delta$ citB::kan citA-3xFLAG spec & GP1287 $\rightarrow$ GP2338 \\
\hline GP2337 & $\begin{array}{l}\operatorname{trpC2} \Delta \text { citRA::lox72 sacA::(phl-PxylA-cre) } \Delta \text { citZ-icd- } \\
\text { mdh::lox72 } \Delta \text { citB::lox72 } \Delta \text { citG::lox72 } \Delta \text { sdhCAB::lox72 }\end{array}$ & GP2335 + 1\% Xylose \\
\hline GP2338 & $\operatorname{trpC2}$ sacA::(phl-PxylA-cre) $\Delta$ citB::kan-lox & LFH-PCR $\rightarrow$ GP2328 \\
\hline GP2339 & $\operatorname{trpC2}$ sacA::(phl-PxylA-cre) $\Delta$ citB::lox72 & GP2338 + 1\% Xylose \\
\hline GP2340 & $\operatorname{trpC2}$ sacA::(phl-P ${ }_{x y \mid A-c r e)} \Delta c i t G:: k a n-l o x$ & LFH-PCR $\rightarrow$ GP2328 \\
\hline GP2341 & $\operatorname{trpC2}$ sacA::(phl-PxylA-cre) $\Delta$ citG::lox72 & GP2340 + 1\% Xylose \\
\hline GP2342 & $\operatorname{trpC2}$ sacA::(phl-PxylA-cre) $\triangle$ sdhCAB::kan-lox & LFH-PCR $\rightarrow$ GP2328 \\
\hline GP2343 & $\operatorname{trpC2}$ sacA::(phl-PxylA-cre) $\triangle$ sdhCAB::lox72 & GP2342 + 1\% Xylose \\
\hline GP2344 & $\operatorname{trpC2}$ sacA::(phl-Pxy|A-cre) $\triangle$ sucCD::kan-lox & LFH-PCR $\rightarrow$ GP2328 \\
\hline GP2345 & $\operatorname{trpC2}$ sacA::(phl-Pxy|A-cre) $\triangle$ sucCD::lox72 & GP2344 + 1\% Xylose \\
\hline GP2346 & $\begin{array}{l}\operatorname{trpC2} \Delta \text { citRA::lox72 sacA::(phl-Pxy|A-cre) } \Delta \text { citZ-icd- } \\
\text { mdh::lox72 } \Delta \text { citB::lox72 } \Delta \text { citG::kan-lox }\end{array}$ & LFH-PCR $\rightarrow$ GP2330 \\
\hline GP2347 & $\operatorname{trpC2}$ lacA::(C-yfp kan) citA-3xFLAG spec & pGP888 $\rightarrow$ GP1287 \\
\hline GP2348 & $\operatorname{trpC2}$ lacA::(Pxyl citZ kan) citA-3xFLAG spec & pGP2261 $\rightarrow$ GP1287 \\
\hline GP2349 & $\operatorname{trpC2}$ lacA::(Pxyl citR kan) citA-3xFLAG spec & pGP2262 $\rightarrow$ GP1287 \\
\hline
\end{tabular}




\begin{tabular}{|c|c|c|}
\hline GP2350 & $\operatorname{trpC2}$ lacA::(PxylcitZ kan) & pGP2261 $\rightarrow 168$ \\
\hline GP2351 & $\operatorname{trpC2}$ lacA::(PxylcitR kan) & pGP2262 $\rightarrow 168$ \\
\hline GP2352 & $\begin{array}{l}\operatorname{trpC2} \Delta \text { citRA::lox72 sacA::(phl-PxylA-cre) } \Delta \text { citZ-icd- } \\
\text { mdh::Iox72 } \Delta \text { citB::lox72 } \Delta \text { citG::lox72 }\end{array}$ & GP2346 + 1\% Xylose \\
\hline GP2353 & $\operatorname{trp} C 2 \Delta \operatorname{citA}::$ cat lacA::(Pxyl citR kan) & pGP2262 $\rightarrow$ GP1282 \\
\hline GP2354 & trpC2 $\triangle$ aapA::tet $\triangle$ gltAB::erm & BP123 $\rightarrow$ GP2377 \\
\hline GP2355 & trpC2 $\Delta$ steT::cat $\Delta g / t A B:: e r m$ & BP123 $\rightarrow$ GP2378 \\
\hline GP2356 & $\begin{array}{l}\operatorname{trpC2} \text { sacA::(phl-PxylA-cre) } \Delta c i t Z-i c d-m d h:: / o x 72 \\
\Delta \text { sucCD::tet }\end{array}$ & PCR GP791 $\rightarrow$ GP2333 \\
\hline GP2357 & $\begin{array}{l}\operatorname{trpC2} \text { sacA::(phl-PxlA-cre) } \Delta \text { citZ-icd-mdh::lox72 } \triangle \text { sucCD::tet } \\
\triangle \text { sdhCAB::kan-lox }\end{array}$ & PCR GP2342 $\rightarrow$ GP2356 \\
\hline GP2358 & $\operatorname{trpC2} \Delta t y r A:: k a n$ & $\mathrm{LFH} \rightarrow 168$ \\
\hline GP2359 & trpC2 citA-3xFLAG spec $\Delta$ citR::aphA3 $\Delta$ citZ::erm & GP1281 $\rightarrow$ GP1289 \\
\hline GP2360 & $\operatorname{trpC2} \triangle$ citRA::erm-lox & $\mathrm{LFH} \rightarrow 168$ \\
\hline GP2361 & $\begin{array}{l}\operatorname{trpC2} \text { sacA::(phl-PxylA-cre) } \Delta c i t Z-i c d-m d h \Delta s u c C D:: t e t \\
\Delta s d h C A B:: 1 o x 72\end{array}$ & GP2357 + 1\% Xylose \\
\hline GP2362 & $\operatorname{trpC2} \Delta t y r A:: k a n \Delta y b e C:: c a t$ & GP1886 $\rightarrow$ GP2358 \\
\hline GP2363 & $\operatorname{trpC2} \Delta$ tyrA::kan $\Delta y \operatorname{tnA}:: s p e c$ & GP1885 $\rightarrow$ GP2358 \\
\hline GP2364 & $\operatorname{trpC2} \Delta$ serA::Tn917 (ermC) $\Delta y \operatorname{tnA}:: s p e c$ & GP1885 $\rightarrow 1 \mathrm{~A} 614$ \\
\hline GP2365 & $\operatorname{trpC2} \Delta$ serA::Tn917 (ermC) $\Delta a l s T:: t e t$ & GP1888 $\rightarrow 1 \mathrm{~A} 614$ \\
\hline GP2366 & $\operatorname{trpC2} \Delta$ serA::Tn917 (ermC) $\Delta y b e C:: c a t$ & GP1886 $\rightarrow 1 \mathrm{~A} 614$ \\
\hline GP2367 & $\operatorname{trpC2} \Delta$ tyrA::kan $\Delta a l s T:: t e t$ & GP1888 $\rightarrow$ GP2358 \\
\hline GP2368 & $\begin{array}{l}\operatorname{trpC2} \text { sacA::(phl-Pxy/A-cre) } \Delta c i t Z-i c d-m d h:: l o x 72 \Delta s u c C D:: t e t \\
\Delta s d h C A B:: / o x 72 \Delta c i t G:: s p c\end{array}$ & PCR GP718 $\rightarrow$ GP2361 \\
\hline
\end{tabular}




\begin{tabular}{|c|c|c|}
\hline GP2369 & MGB874 $\Delta$ tyrA::kan & PCR GP2358 $\rightarrow$ MGB874 \\
\hline GP2370 & $\operatorname{trpC2}$ asnS ( $\triangle \mathrm{bp} 473$, stop aa 195$)$ & $\begin{array}{l}\text { Suppressor mutant } 168 \text { on L- } \\
\text { Aspartic acid } \beta \text {-hydroxamate } \\
\text { (WGS) }\end{array}$ \\
\hline
\end{tabular}

\begin{tabular}{ll}
\hline GP2371 $\quad \operatorname{trpC2}$ Promoter asnS $(\triangle \mathrm{bp}-13)$ & Suppressor mutant 168 on L- \\
& Aspartic acid $\beta$-hydroxamate \\
& (PCR)
\end{tabular}

GP2372 trpC2 asnS ( $\Delta \mathrm{bp} 172$, stop aa 58) Suppressor mutant 168 on L-

Aspartic acid $\beta$-hydroxamate

(PCR)

\begin{tabular}{lll}
\hline GP2373 & $\operatorname{trpC2} \Delta$ yodF::neo asnS (bp $805 \mathrm{G} \rightarrow \mathrm{A}$, stop aa 269) & $\begin{array}{l}\text { Suppressor mutant GP1887 } \\
\text { on L-Aspartic acid } \beta \text { - } \\
\text { hydroxamate (PCR) }\end{array}$ \\
\hline GP2374 & $\operatorname{trpC2~} \Delta$ gltT::ermC $\triangle$ yodF::neo & GP1887 $\rightarrow$ GP2247 \\
\hline GP2375 & $\operatorname{trpC2~ansA~}(\Delta b p$ 367, stop aa 157) & Suppressor mutant 168 on L- \\
& & Aspartic acid $\beta$-hydroxamate \\
& & (WGS)
\end{tabular}

\begin{tabular}{lll}
\hline GP2376 & $\operatorname{trpC2} \Delta$ tyrA::kan yqiK $(b p$ 677 $T \rightarrow G$, aa 226 $D \rightarrow \mathrm{V})$ & Suppressor mutant GP2358 \\
& & on C-glc-tyrosin (WGS) \\
\hline GP2377 & $\operatorname{trpC2~} \triangle$ aapA::tet & LFH $\rightarrow 168$
\end{tabular}

\begin{tabular}{lll}
\hline GP2378 & $\operatorname{trpC2} \Delta$ steT::cat & LFH $\rightarrow 168$ \\
& & \\
\hline GP2379 & $\operatorname{trpC2} \Delta y f n A:: k a n$ & LFH $\rightarrow 168$
\end{tabular}

\begin{tabular}{lll}
\hline GP2380 & $\operatorname{trpC2}$ yqiK (D226V)-cat & LFH $\rightarrow$ GP2358 \\
& & \\
\hline GP2381 & $\operatorname{trpC2~} \triangle$ aapA::tet serA::Tn917 (ermC) & 1A614 $\rightarrow$ GP2377
\end{tabular}

\begin{tabular}{lll}
\hline GP2382 $\operatorname{trpC2} \Delta$ steT::cat serA::Tn917 (ermC) & 1A614 $\rightarrow$ GP2378
\end{tabular}

\begin{tabular}{lll}
\hline GP2383 & $\operatorname{trpC2} \Delta y f n A:: k a n$ serA::Tn917 (ermC) & 1A614 $\rightarrow$ GP2379 \\
& & \\
\hline GP2384 & trpC2 $\Delta$ yqiK::cat & LFH $\rightarrow 168$
\end{tabular}




\begin{tabular}{|c|c|c|}
\hline GP2385 & $\operatorname{trpC2} \Delta y v e A:: c a t$ & $\mathrm{LFH} \rightarrow 168$ \\
\hline GP2386 & $\begin{array}{l}\operatorname{trpC2} \Delta \text { yodF::kan tcyK (bp } 480 \mathrm{C} \rightarrow \mathrm{T} \text {, aa } 159 \mathrm{~V} \rightarrow \mathrm{I}) \mathrm{P}(\text { infC- } \\
\text { rpml-rp/T-ysdA) }(\Delta \mathrm{bp}-171)\end{array}$ & $\begin{array}{l}\text { Suppressor mutant GP1887 } \\
\text { on L-Aspartic acid } \beta \text { - } \\
\text { hydroxamate (WGS) }\end{array}$ \\
\hline GP2387 & $\operatorname{trpC2} \Delta y v e A:: c a t \Delta g / t T:: e r m C$ & GP2247 $\rightarrow$ GP2385 \\
\hline GP2388 & trpC2 yqiK (D226V)-cat $\Delta$ tyrA::kan & PCR GP2358 $\rightarrow$ GP2380 \\
\hline GP2389 & $\operatorname{trpC2} \Delta y q i K:: c a t \Delta t y r A:: k a n$ & PCR GP2358 $\rightarrow$ GP2384 \\
\hline GP2390 & $\operatorname{trpC2} \Delta y f n A:: k a n \Delta g / t A B:: e r m$ & PCR BP123 $\rightarrow$ GP2379 \\
\hline GP2391 & $\operatorname{trpC2} \Delta y s d A:: k a n$ & $\mathrm{LFH} \rightarrow 168$ \\
\hline GP2392 & $\operatorname{trpC2} \Delta$ serA::zeo & $\mathrm{LFH} \rightarrow 168$ \\
\hline GP2393 & $\operatorname{trpC2} \Delta a a p A:: t e t \Delta y f n A:: k a n$ & GP2379 $\rightarrow$ GP2377 \\
\hline GP2394 & $\operatorname{trpC2} \Delta$ aapA::tet $\triangle y f n A:: k a n \Delta s t e T:: c a t$ & GP2378 $\rightarrow$ GP2393 \\
\hline GP2395 & $\operatorname{trpC2} \Delta y h j B:: t e t$ & $\mathrm{LFH} \rightarrow 168$ \\
\hline GP2396 & $\operatorname{trpC2} \Delta y b x G:: c a t$ & $\mathrm{LFH} \rightarrow 168$ \\
\hline GP2397 & $\operatorname{trpC2} \Delta y f n A:: k a n \Delta s t e T:: c a t$ & GP2378 $\rightarrow$ GP2379 \\
\hline GP2398 & $\operatorname{trpC2} \triangle$ aapA::tet $\Delta$ steT::cat & GP2378 $\rightarrow$ GP2377 \\
\hline GP2399 & $\operatorname{trpC2} \Delta y$ hjB::tet $\Delta$ serA::Tn917 (ermC) & $1 \mathrm{~A} 614 \rightarrow \mathrm{GP} 2395$ \\
\hline GP2400 & $\operatorname{trpC2} \Delta y h j B::$ tet $\Delta t y r A:: k a n$ & GP2358 $\rightarrow$ GP2395 \\
\hline GP2926 & $\operatorname{trpC2} \Delta y h j B::$ tet $\Delta g l t T:: e r m$ & GP2247 $\rightarrow$ GP2395 \\
\hline GP2927 & $\operatorname{trpC2} \Delta y b x G:: c a t \Delta s e r A:: T n 917$ (ermC) & $1 \mathrm{~A} 614 \rightarrow \mathrm{GP} 2396$ \\
\hline GP2928 & $\operatorname{trpC2} \Delta y b x G:: c a t \Delta t y r A:: k a n$ & GP2358 $\rightarrow$ GP2396 \\
\hline
\end{tabular}




\begin{tabular}{|c|c|c|}
\hline GP2929 & $\operatorname{trpC2} \Delta y b x G:: c a t \Delta g / t T:: e r m$ & GP2247 $\rightarrow$ GP2396 \\
\hline GP2930 & $\operatorname{trpC2} \Delta y d g F:: c a t$ & LFH $\rightarrow 168$ \\
\hline GP2931 & $\operatorname{trpC2} \Delta y d g F::$ cat $\Delta g \mid t T:: e r m$ & GP2247 $\rightarrow$ GP2930 \\
\hline GP2932 & $\operatorname{trpC2} \Delta y d g F:: c a t \Delta t y r A:: k a n$ & GP2358 $\rightarrow$ GP2930 \\
\hline GP2933 & $\operatorname{trpC2} \Delta y d g F:: c a t \Delta s e r A:: T n 917$ (ermC) & $1 \mathrm{~A} 614 \rightarrow \mathrm{GP} 2930$ \\
\hline \multirow[t]{3}{*}{ GP2934 } & $\operatorname{trpC2}$ asnS ( $\triangle \mathrm{bp} 1014-1023$, frameshift, stop aa 342$)$ & Suppressor mutant 168 on L- \\
\hline & & Aspartic acid $\beta$-hydroxamate \\
\hline & & (PCR) \\
\hline \multirow[t]{3}{*}{ GP2935 } & $\operatorname{trpC2}$ asnS (bp $644 \mathrm{~T} \rightarrow \mathrm{C}$, aa $215 \mathrm{H} \rightarrow \mathrm{R})$ & Suppressor mutant 168 on L- \\
\hline & & Aspartic acid $\beta$-hydroxamate \\
\hline & & (PCR) \\
\hline
\end{tabular}

\begin{tabular}{|c|c|c|}
\hline GP2936 & $\begin{array}{l}\operatorname{trpC2} \text { asnS (bp } 369 \mathrm{G} \rightarrow \mathrm{T} \text {, stop aa 369) yvoD (bp } 239 \mathrm{G} \rightarrow \mathrm{T}, \\
\text { aа } 239 \mathrm{~A} \rightarrow \mathrm{D})\end{array}$ & $\begin{array}{l}\text { Suppressor mutant } 168 \text { on L- } \\
\text { Aspartic acid } \beta \text {-hydroxamate } \\
\text { (WGS) }\end{array}$ \\
\hline GP2937 & $\operatorname{trpC2}$ asnS ( $\Delta \mathrm{bp} 1207-1210$, frameshift, stop aa 405) & $\begin{array}{l}\text { Suppressor mutant } 168 \text { on } \mathrm{L} \text { - } \\
\text { Aspartic acid } \beta \text {-hydroxamate } \\
\text { (PCR) }\end{array}$ \\
\hline
\end{tabular}

\begin{tabular}{|c|c|c|}
\hline GP2938 & $\operatorname{trpC2} \Delta g / t T:: e r m$, asnS ( $\triangle \mathrm{bp} 900-1229$, in-frame deletion) & $\begin{array}{l}\text { Suppressor mutant GP2247 } \\
\text { on L-Aspartic acid } \beta \text { - } \\
\text { hydroxamate (PCR) }\end{array}$ \\
\hline GP2939 & $\begin{array}{l}\operatorname{trpC2} \Delta g / t T:: e r m, \text { asnS (duplication of bp 147-328; } \\
\text { in-frame) }\end{array}$ & $\begin{array}{l}\text { Suppressor mutant GP2247 } \\
\text { on L-Aspartic acid } \beta \text { - } \\
\text { hydroxamate (PCR) }\end{array}$ \\
\hline GP2940 & $\operatorname{trpC2} \Delta y b e C:: c a t \Delta t h r R:: e r m$ & BKE27910 $\rightarrow$ GP1886 \\
\hline GP2941 & $\operatorname{trpC2} \Delta$ serA::zeo $\triangle y b e C:: c a t$ & GP1886 $\rightarrow$ GP2392 \\
\hline GP2942 & $\operatorname{trpC2} \Delta$ serA::zeo $\Delta$ thrR::erm & BKE27910 $\rightarrow$ GP2392 \\
\hline GP2943 & $\operatorname{trpC2} \Delta y v o D:: c a t$ & LFH $\rightarrow 168$ \\
\hline GP2944 & $\operatorname{trpC2} \Delta y v o D:: c a t \Delta g l t T:: e r m$ & GP2247 $\rightarrow$ GP2943 \\
\hline
\end{tabular}




\begin{tabular}{|c|c|c|}
\hline GP2945 & $\operatorname{trpC2} \Delta$ steT::kan & $\mathrm{LFH} \rightarrow 168$ \\
\hline GP2946 & $\operatorname{trpC2} \Delta y b e C:: c a t \Delta s t e T:: k a n$ & GP2946 $\rightarrow$ GP1886 \\
\hline GP2947 & $\operatorname{trpC2} \Delta y b e C::$ cat $x k d E::(N-y f p$ ermR) & GP1171 $\rightarrow$ GP1886 \\
\hline GP2948 & $\operatorname{trpC2} \Delta y b e C:: c a t$ xkdE::(Pxyl-ybeC ermC) & GP3080 $\rightarrow$ GP1886 \\
\hline GP2949 & $\operatorname{trpC2} \Delta y b e C:: k a n \Delta b c a P:: e r m$ & BKE09460 $\rightarrow$ GP2786 \\
\hline GP2950 & $\operatorname{trpC2} \Delta y b e C:: k a n \Delta b c a P:: e r m \Delta y b x G:: c a t$ & GP2396 $\rightarrow$ GP2949 \\
\hline GP2951 & $\operatorname{trpC2} \Delta y b e C:: k a n \Delta y b x G:: c a t$ & GP2396 $\rightarrow$ GP2786 \\
\hline GP2952 & $\operatorname{trpC2} \Delta b c a P:: e r m \Delta y b x G:: c a t$ & GP2396 $\rightarrow$ BKE09460 \\
\hline GP2953 & $\operatorname{trpC2} \Delta y b e C:: k a n \Delta b c a P:: e r m \Delta$ serA::zeo & GP2392 $\rightarrow$ GP2949 \\
\hline GP2954 & $\operatorname{trpC2} \Delta b c a P:: e r m \Delta y b x G:: c a t \Delta$ serA::zeo & GP2392 $\rightarrow$ GP2952 \\
\hline GP2955 & $\operatorname{trpC2} \Delta y b e C:: k a n \Delta b c a P:: e r m \Delta y b x G:: c a t \Delta s e r A:: z e o$ & GP2392 $\rightarrow$ GP2950 \\
\hline GP2956 & $\operatorname{trpC2} \Delta y b x G::$ cat $\Delta$ serA::zeo & GP2392 $\rightarrow$ GP2396 \\
\hline GP2957 & $\operatorname{trpC2} \Delta y b e C:: k a n \Delta s e r A:: z e o$ & GP2392 $\rightarrow$ GP2786 \\
\hline GP2958 & $\operatorname{trpC2} \Delta y b e C:: k a n \Delta y b x G:: c a t \Delta s e r A:: z e o$ & GP2392 $\rightarrow$ GP2951 \\
\hline GP2959 & $\operatorname{trpC2} \triangle b c a P:: e r m \Delta$ serA::zeo & GP2392 $\rightarrow$ BKE09460 \\
\hline GP2960 & $\operatorname{trpC2}$ amyE::(aapA-lacZ cat) & pGP2273 $\rightarrow 168$ \\
\hline GP2961 & $\operatorname{trpC2}$ amyE::(ansB-lacZ cat) & pGP2274 $\rightarrow 168$ \\
\hline GP2962 & $\operatorname{trpC2}$ amyE::(steT-lacZ cat) & $\mathrm{pGP} 2275 \rightarrow 168$ \\
\hline GP2963 & $\operatorname{trpC2}$ amyE::(ytnA-lacZ cat) & pGP2276 \\
\hline
\end{tabular}




\begin{tabular}{|c|c|c|}
\hline GP2964 & $\operatorname{trpC2}$ amyE::(yfnA-lacZ cat) & pGP2278 $\rightarrow 168$ \\
\hline GP2965 & $\operatorname{trpC2}$ amyE::(ybeC-lacZ cat) & pGP2287 $\rightarrow 168$ \\
\hline GP2966 & $\operatorname{trpC2}$ amyE::(sdaAB* C7OA-lacZ cat) & pGP2294 $\rightarrow 168$ \\
\hline GP2967 & $\operatorname{trpC2}$ amyE::(sdaAB-lacZ cat) & pGP2295 $\rightarrow 168$ \\
\hline GP2968 & $\operatorname{trpC2}$ amyE::(hom* G56T-lacZ cat) & pGP2296 $\rightarrow 168$ \\
\hline GP2969 & $\operatorname{trpC2} \Delta$ serA::zeo; thrR ( $\Delta$ bp 90, stop aa 36) & $\begin{array}{l}\text { Suppressor mutant GP2392 } \\
\text { on C-glc-Serine (WGS) }\end{array}$ \\
\hline GP2970 & $\operatorname{trpC2}$ duplication (yokD [bp 43-819] -thyA [bp 510-795]) & $\begin{array}{l}\text { Suppressor mutant } 168 \text { on C- } \\
\text { glc-Serine (WGS) }\end{array}$ \\
\hline GP2971 & $\operatorname{trpC2} \triangle y b e C:: k a n$ Promoter sdaAB* $[b p-70 C \rightarrow A]$ & $\begin{array}{l}\text { Suppressor mutant GP1886 } \\
\text { on C-glc-Serine (WGS) }\end{array}$ \\
\hline GP2972 & $\operatorname{trpC2} \Delta y b e C:: k a n$ Promoter hom* [bp -56 G $\rightarrow T]$ & $\begin{array}{l}\text { Suppressor mutant GP1886 } \\
\text { on C-glc-Serine (WGS) }\end{array}$ \\
\hline GP2973 & 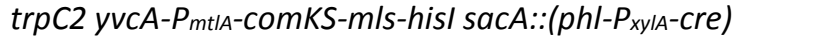 & pJK195 $\rightarrow$ GP2618 \\
\hline GP2974 & $\begin{array}{l}\operatorname{trpC2} \text { yvcA-Pmt/A-comKS-m/s-hisl sacA::(phl-Pxy/A-cre) } \Delta \text { citZ- } \\
\text { icd-mdh::lox72 }\end{array}$ & $\begin{array}{l}\text { PCR GP2331 } \rightarrow \text { GP2973 + 1\% } \\
\text { Xylose }\end{array}$ \\
\hline GP2975 & $\begin{array}{l}\operatorname{trpC2} \text { yvcA-Pmt/A-comKS-m/s-hisl sacA::(phl-PxylA-cre) } \Delta \text { citZ- } \\
\text { icd-mdh::lox72 } \Delta \text { sucCD::tet }\end{array}$ & PCR GP791 $\rightarrow$ GP2974 \\
\hline GP3024 & $\begin{array}{l}\operatorname{trpC2} \text { yvcA-Pmt/A-comKS-m/s-hisl sacA::(phl-PxylA-cre) } \Delta \text { citZ- } \\
\text { icd-mdh::lox72 } \Delta \text { sucCD::tet } \Delta \text { sdhCAB::lox72 }\end{array}$ & $\begin{array}{l}\text { PCR GP2342 } \rightarrow \text { GP2975 + 1\% } \\
\text { Xylose }\end{array}$ \\
\hline GP3025 & $\begin{array}{l}\operatorname{trpC2} \text { yvcA-Pmt/A-comKS-m/s-hisl sacA::(phl-PxylA-cre) } \Delta \text { citZ- } \\
\text { icd-mdh::lox72 } \Delta \text { sucCD::tet } \Delta \text { sdhCAB::lox72 } \Delta \text { citG::spec }\end{array}$ & PCR GP718 $\rightarrow$ GP3024 \\
\hline GP3026 & 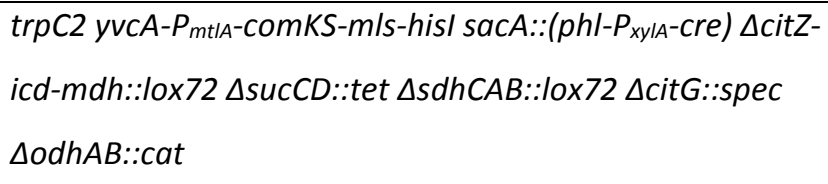 & PCR GP1276 $\rightarrow$ GP3025 \\
\hline GP3027 & $\begin{array}{l}\operatorname{trpC2} \text { yvcA-Pmt/A-comKS-m/s-hisl sacA::(phl-PxylA-cre) } \Delta \text { citZ- } \\
\text { icd-mdh::lox72 } \Delta \text { sucCD::tet } \Delta \text { sdhCAB::lox72 } \Delta \text { citG::spec } \\
\Delta \text { odhAB::cat } \Delta \text { citB::lox72 }\end{array}$ & $\begin{array}{l}\text { PCR GP2338 } \rightarrow \text { GP3026 +1\% } \\
\text { Xylose }\end{array}$ \\
\hline GP3028 & $\begin{array}{l}\text { trpC2 yvcA-Pmt/A-comKS-m/s-hisl sacA::(phl-PxylA-cre) } \Delta \text { citZ- } \\
\text { icd-mdh::lox72 } \Delta \text { sucCD::tet } \triangle \text { sdhCAB::lox72 } \Delta \text { citG::spec } \\
\Delta o d h A B:: \text { cat } \Delta \text { citB::lox72 } \Delta \text { citRA::lox72 }\end{array}$ & $\begin{array}{l}\text { PCR GP1753 } \rightarrow \text { GP3027 +1\% } \\
\text { Xylose }\end{array}$ \\
\hline
\end{tabular}




\begin{tabular}{|c|c|c|}
\hline \multirow[t]{2}{*}{ GP3029 } & $\operatorname{trpC2}$ yvcA-P $P_{m t / A}-c o m K S-m / s-h i s /$ sacA::(phl-PxylA-cre) & PCR GP2342 $\rightarrow$ GP2973 +1\% \\
\hline & $\triangle s d h C A B:: / o x 72$ & Xylose \\
\hline GP3030 & $\operatorname{trpC2} \Delta t h r C:: s p e c$ & $\mathrm{LFH} \rightarrow 168$ \\
\hline GP3031 & $\operatorname{trpC2} \Delta b c a P:: e r m \Delta t h r C:: s p e c$ & GP3030 $\rightarrow$ BKE09460 \\
\hline GP3032 & $\operatorname{trpC2} \Delta y b x G:: c a t \Delta t h r C:: s p e c$ & GP3030 $\rightarrow$ GP2396 \\
\hline
\end{tabular}

\begin{tabular}{|c|c|c|}
\hline GP3033 & $\operatorname{trpC2} \Delta y b e C:: k a n \Delta t h r C:: s p e c$ & GP3030 $\rightarrow$ GP2786 \\
\hline GP3034 & $\operatorname{trpC2} \Delta b c a P:: e r m \Delta y b x G:: c a t \Delta t h r C:: s p e c$ & GP3030 $\rightarrow$ GP2952 \\
\hline GP3035 & $\operatorname{trpC2} \Delta b c a P:: e r m \Delta y b e C:: k a n \Delta t h r C:: s p e c$ & GP3030 $\rightarrow$ GP2949 \\
\hline GP3036 & $\operatorname{trpC2} \Delta y b e C:: k a n \Delta y b x G:: c a t \Delta t h r C:: s p e c$ & GP3030 $\rightarrow$ GP2951 \\
\hline GP3037 & $\operatorname{trpC2} \Delta y b e C:: k a n \Delta y b x G:: c a t \Delta b c a P:: e r m \Delta$ & GP3030 $\rightarrow$ GP2950 \\
\hline
\end{tabular}

\begin{tabular}{lll}
\hline GP3038 & $\operatorname{trpC2} \Delta y v b W:: c a t$ & LFH $\rightarrow 168$ \\
& & \\
\hline GP3039 & $\operatorname{trpC2} \triangle$ yecA::cat & LFH $\rightarrow 168$
\end{tabular}

\begin{tabular}{lll}
\hline GP3040 $\quad$ trpC2 $\Delta y b g F:: c a t$ & LFH $\rightarrow 168$
\end{tabular}

\begin{tabular}{lll}
\hline GP3041 & trpC2 $\Delta$ yodF::cat $\Delta g / t$ : :erm & GP2247 $\rightarrow$ GP1887 \\
\hline GP3042 & trpC2 $\Delta$ yodF::cat $\Delta$ tyrA:: kan & GP2358 $\rightarrow$ GP1887 \\
& & \\
\hline GP3043 trpC2 $\Delta y v b W:: c a t \Delta g l t T:: e r m$ & GP2247 $\rightarrow$ GP3038
\end{tabular}

$\begin{array}{lll}\text { GP3044 } \operatorname{trpC2} \Delta y v b W:: c a t \Delta t y r A:: k a n & \text { GP2358 } \rightarrow \text { GP3038 }\end{array}$

\begin{tabular}{lcc}
\hline GP3045 & trpC2 $\Delta y e c A::$ cat $\Delta g l t T:: e r m$ & GP2247 $\rightarrow$ GP3039 \\
\hline GP3046 & trpC2 $\Delta y e c A::$ cat $\Delta$ tyrA:: kan & GP2358 $\rightarrow$ GP3039 \\
& & \\
\hline GP3047 & trpC2 $\Delta y b g F::$ cat $\Delta g l t T::$ erm & GP2247 $\rightarrow$ GP3040
\end{tabular}




\begin{tabular}{|c|c|c|}
\hline GP3048 & $\operatorname{trpC2} \Delta y b g F:: c a t \Delta t y r A::$ kan & GP2358 $\rightarrow$ GP3040 \\
\hline GP3049 & $\operatorname{trpC2} \Delta$ serA::zeo ybeC (bp $1564 \mathrm{G} \rightarrow \mathrm{T}$, stop aa 522) & $\begin{array}{l}\text { Suppressor mutant of GP2392 } \\
\text { on high serine concentrations } \\
\text { (PCR) }\end{array}$ \\
\hline GP3050 & $\operatorname{trpC2} \Delta$ serA::zeo ybeC ( $\Delta \mathrm{bp} 307-1014$, in-frame deletion) & $\begin{array}{l}\text { Suppressor mutant of GP2392 } \\
\text { on high serine concentrations } \\
\text { (PCR) }\end{array}$ \\
\hline PG29 & PG18 $\triangle m h q N O P$ & pGP2093 $\rightarrow$ PG18 \\
\hline PG30 & PG29 $\Delta(y u z G-s u f A)$ & pGP2094 $\rightarrow$ PG29 \\
\hline PG31 & PG30 restored wild type pit allele & pJOE3256 $\rightarrow$ PG30 \\
\hline PG32 & PG31 $\Delta y t r H:: p g i-f b a A-c a t$ & PCR product $\rightarrow$ PG31 \\
\hline PG33 & PG32 $\Delta$ (cat-ytrl-ytzJ)::ptsGHI-kan & PCR product $\rightarrow$ PG32 \\
\hline PG34 & PG33 $\Delta(y c g Q-y c k E)$ & pGP2098 $\rightarrow$ PG33 \\
\hline PG35 & PG34 $\Delta(y v a M-y v b K)$ & pGP2088 $\rightarrow$ PG34 \\
\hline PG36 & PG35 $\Delta($ nhaX-yhaX) & pGP2073 $\rightarrow$ PG35 \\
\hline PG37 & $\mathrm{PG} 36 \Delta(g / p Q-y c b K)$ & pGP2270 $\rightarrow$ PG36 \\
\hline PG38 & PG37 $\Delta(y q j F-y q j G)$ & pGP2282 $\rightarrow$ PG37 \\
\hline PG39 & PG38 $\Delta(y d d N-y d f M)$ & pGP2283 $\rightarrow$ PG38 \\
\hline
\end{tabular}




\subsubsection{Isolated suppressor mutants of this work}

\begin{tabular}{|c|c|c|}
\hline Strain & Phenotype relevant mutation & Remarks \\
\hline \multicolumn{3}{|c|}{ 168, C-glc $244 \mu \mathrm{M}$ serine } \\
\hline GP2970 & duplication (yokD[bp 43-819] -thyA[bp 510-795]) & WGS \\
\hline \multicolumn{3}{|c|}{ 168, C-glc $1 \mathrm{mM}$ serine } \\
\hline GP2324 & $\begin{array}{l}\text { ybeC }(\Delta \mathrm{bp} 340, \text { stop aa } 125) \\
\text { duplication (yokD[bp 43-819] -thyA[bp 510-795]) }\end{array}$ & WGS \\
\hline GP2325 & ybeC ( $\Delta$ bp 974, stop aa 350$)$ & PCR ybec \\
\hline \multicolumn{3}{|c|}{ GP2392 ( $\Delta$ serA::zeo), C-glc $244 \mu \mathrm{M}$ serine } \\
\hline GP2969 & thrR ( $\Delta \mathrm{bp} 90$, stop aa 36$)$ & WGS \\
\hline GP3049 & ybeC (bp $1564 \mathrm{G} \rightarrow \mathrm{T}$, stop aa 522) & PCR ybec \\
\hline GP3050 & $y b e c(\Delta \mathrm{bp} 307-1014$, in-frame deletion) & PCR ybec \\
\hline \multicolumn{3}{|c|}{ GP1886 ( $\triangle$ ybeC::cat), C-glc $10 \mathrm{mM}$ serine } \\
\hline GP2971 & Promoter $s d a A B^{*}[b p-70 C \rightarrow A]$ & WGS \\
\hline \multicolumn{3}{|c|}{ GP1886 (4ybeC::cat), C-glc $17 \mathrm{mM}$ serine } \\
\hline GP2972 & Promoter hom* $[b p-56 G \rightarrow T]$ & WGS \\
\hline \multicolumn{3}{|c|}{ 168, C-glc L-aspartic acid $\beta$-hydroxamate } \\
\hline GP2370 & asnS ( $\Delta$ bp 473, stop aa 195$)$ & WGS \\
\hline GP2371 & Promoter asns ( $\Delta \mathrm{bp}-13)$ & PCR asns \\
\hline GP2372 & $\operatorname{asnS}(\Delta \mathrm{bp} 172$, stop aa 58$)$ & PCR asns \\
\hline GP2934 & asns ( $\Delta$ bp 1014-1023, frameshift, stop aa 342) & PCR asns \\
\hline GP2935 & asnS (bp $644 \mathrm{~T} \rightarrow \mathrm{C}$, aa $215 \mathrm{H} \rightarrow \mathrm{R})$ & PCR asns \\
\hline GP2936 & asnS (bp $369 \mathrm{G} \rightarrow \mathrm{T}$, stop aa 369) yvoD (bp $239 \mathrm{G} \rightarrow \mathrm{T}$, aa $239 \mathrm{~A} \rightarrow \mathrm{D})$ & WGS \\
\hline GP2937 & asnS ( $\Delta$ bp 1207-1210, frameshift, stop aa 405$)$ & PCR asns \\
\hline \multicolumn{3}{|c|}{ GP1887 (DyodF::kan), C-glc L-aspartic acid $\beta$-hydroxamate } \\
\hline GP2373 & asnS (bp 805 G $\rightarrow$ A, stop aa 269) & PCR asns \\
\hline \multicolumn{3}{|c|}{ GP2247 ( $\Delta g / t T:: e r m)$, C-glc L-aspartic acid $\beta$-hydroxamate } \\
\hline GP2938 & asnS ( $\Delta$ bp $900-1229$, in-frame deletion & PCR asns \\
\hline GP2939 & asnS (duplication of bp 147-328, in-frame) & PCR asns \\
\hline
\end{tabular}

\subsubsection{B. subtilis strains used in this work}

\begin{tabular}{lll}
\hline Strain & Genotype & Reference/ Construction \\
& & \\
\hline $1 \mathrm{~A} 614$ & $\operatorname{trpC2} \operatorname{ser} A:: \operatorname{Tn} 917(\mathrm{ermC})$ & Vandeyar and Zahler, 1986
\end{tabular}




\begin{tabular}{|c|c|c|}
\hline $1 \mathrm{~A} 773$ & pheA1 trpC2 thrC::cat & Vandeyar and Zahler, 1986 \\
\hline BKE09460 & $\operatorname{trpC2} \Delta b c a P:: e r m$ & Koo et al., 2017 \\
\hline BKE27910 & trpC2 $\Delta$ thrR::erm & Koo et al., 2017 \\
\hline \multirow[t]{2}{*}{ BKG9 } & $\operatorname{trpC2} \triangle$ spoOA::kan & Katrin Gunka, Medical \\
\hline & & Microbiology Göttingen \\
\hline BP123 & $\operatorname{trpC2}$ gltAB::ermC & Victoria Keidel, AG Commichau \\
\hline BP557 & trpC2 $\Delta$ thrR::ermC amyE::(Phom * G56T-lacZ cat) & Rosenberg et al., 2016 \\
\hline BP558 & $\operatorname{trpC2}$ amyE::(Phom ${ }^{*}$ G56T-lacZ cat $)$ & Rosenberg et al., 2016 \\
\hline BP562 & $\operatorname{trpC2}$ amyE::(PthrD $*$ G56T-lacZ cat $)$ & Rosenberg et al., 2016 \\
\hline BP563 & trpC2 $\Delta$ thrR::ermC amyE::(PthrD* G56T-lacZ cat) & Rosenberg et al., 2016 \\
\hline GP718 & $\operatorname{trpC2} \Delta$ citG::spc amyE::(gltA-lacZ aphA3) & Commichau, 2006 \\
\hline
\end{tabular}

\begin{tabular}{lll}
\hline GP791 & $\operatorname{trpC2} \Delta$ sucCD ::tet & Zschiedrich, 2014 \\
& & \\
\hline GP1171 & $\operatorname{trpC2}$ xkdE::(N-yfp ermC) & Gunka, 2011
\end{tabular}

\begin{tabular}{lll}
\hline GP1173 & $\operatorname{trpC2}$ lacA::(C-yfp kan) & Diethmaier et al., 2011 \\
\hline GP1276 & $\operatorname{trpC2~} \triangle$ odhAB::cat & Zschiedrich, 2014 \\
\hline GP1281 & $\operatorname{trpC2~} \triangle$ citZ::erm & Zschiedrich, 2014
\end{tabular}

GP1282 trpC2 $\triangle$ citA::cat $\quad$ Zschiedrich, 2014

\begin{tabular}{lll}
\hline GP1283 & $\operatorname{trpC2} \triangle$ citR::aphA3 & Zschiedrich, 2014 \\
\hline GP1284 & $\operatorname{trpC2~} \Delta$ citZ::erm $\triangle$ citA::cat & Zschiedrich, 2014 \\
\hline GP1285 & $\operatorname{trpC2~} \triangle$ citZ::erm $\triangle$ citR::aphA3 & Zschiedrich, 2014
\end{tabular}




\begin{tabular}{lll}
\hline GP1286 & $\operatorname{trpC2} \Delta$ citA::cat $\triangle$ citR::aphA3 & Zschiedrich, 2014 \\
\hline GP1287 & $\operatorname{trpC2~citA-3xFLAG~spec~}$ & Zschiedrich, 2014 \\
\hline GP1288 & $\operatorname{trpC2~citA-3xFLAG~spec~} \Delta$ citZ::erm & Zschiedrich, 2014 \\
\hline GP1289 & $\operatorname{trpC2~citA-3xFLAG~spec~} \Delta$ citR::aphA3 & Zschiedrich, 2014 \\
\hline GP1752 & $\operatorname{trpC2~dnaE-pgi-fbaA-cat-ytrl~}$ & Zschiedrich, 2014 \\
\hline GP1753 & $\operatorname{trpC2~} \Delta$ citRA::aphA3-lox & Zschiedrich, 2014
\end{tabular}

GP1755 $\quad \operatorname{trpC2} \triangle$ citRA sacA::(phl-PxylA-cre) Zschiedrich, 2014

\begin{tabular}{lll}
\hline GP1757 & $\operatorname{trpC2}$ dnaE-pgi-fbaA-ptsGHI-aphA3-ackA $\Delta y$ trl- & Zschiedrich, 2014 \\
& moaB::aphA3 & \\
\hline GP1885 & $\operatorname{trpC2} \Delta y \operatorname{tnA}:: s p e c$ & Reuß, 2017 \\
& & \\
\hline GP1886 & $\operatorname{trpC2} \Delta y b e C::$ cat & Reuß, 2017
\end{tabular}

\begin{tabular}{lll}
\hline GP1887 & trpC2 $\Delta$ yodF::neo & Reuß, 2017 \\
& & \\
\hline GP1888 & trpC2 $\Delta$ alsT::tet & Reuß, 2017
\end{tabular}

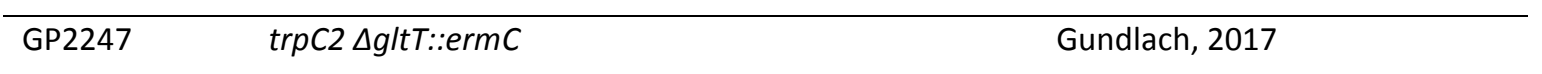

\begin{tabular}{lll}
\hline GP2618 $\operatorname{trpC2} y v c A-P_{m t / A}$-comKS-m/s-hisl & Martin Benda, PhD
\end{tabular}

\begin{tabular}{lll}
\hline GP2786 & $\operatorname{trpC2} \Delta y b e C:: k a n$ & Larissa Krüger, PhD \\
& & \\
\hline GP3080 & $\operatorname{trpC2} x k d E:: P x y l-y b e C$ ermC & Larissa Krüger, PhD
\end{tabular}

\begin{tabular}{lll}
\hline IIG-Bs168-1 trpC2 manPA::ermC & Rahmer et al., 2015
\end{tabular}

\begin{tabular}{lll}
\hline MGB874 & Genome reduced strain of $21 \%$ & Morimoto et al., 2008 \\
\hline PG10 & MiniBacillus genome reduced strain of 34.54\% & Reuß et al., 2017
\end{tabular}




\begin{tabular}{lll}
\hline PG18 & MiniBacillus genome reduced strain of 36.61\% & Reuß, 2017 \\
\hline$\Delta 6$ & deletion of 6 prophaes and AT-rich islands & Westers et al., 2003
\end{tabular}

\subsubsection{E. coli strains used in this work}

\begin{tabular}{|c|c|c|}
\hline Strain & Genotype & Reference/ Construction \\
\hline JM109 & $\begin{array}{l}\text { endA1, recA1, gyrA96, thi, hsdR17 }\left(\mathrm{r}_{\mathrm{k}}{ }^{-},\right. \\
\left.\mathrm{m}_{\mathrm{k}}{ }^{+}\right) \text {, relA1, supE44, } \Delta(\text { /ac-proAB), } \\
{\left[\mathrm{F}^{\prime} \text { traD36, proAB, laq }{ }^{9} \mathrm{Z} \Delta \mathrm{M} 15\right]}\end{array}$ & Yanisch-Perron et al. (1985) \\
\hline XL1 blue & $\begin{array}{l}\text { recA1 endA1 gyrA96 thi-1 hsdR17 supE44 relA1 lac }\left[F^{\prime}\right. \\
\text { proAB lacla } Z \triangle M 15 \text { Tn10 (Tetr) }]\end{array}$ & $\begin{array}{l}\text { Stratagene, Woodcock et al. } \\
\text { (1989) }\end{array}$ \\
\hline $\mathrm{DH} 5 \alpha$ & 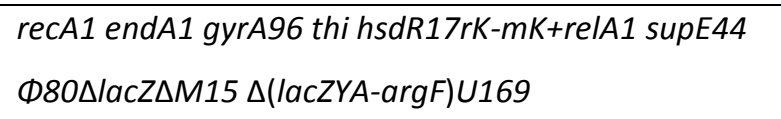 & Sambrook et al., 1989 \\
\hline $\mathrm{BL} 21$ & $\begin{array}{l}\text { B(834)-derivate F-lon ompT hsdS }(r B m B) g a l \\
d c m[D E 3]\end{array}$ & Novagen, Sambrook et al., 1989 \\
\hline
\end{tabular}

\subsection{Plasmids}

\subsubsection{Plasmids constructed in this work}

\begin{tabular}{lll}
\hline Plasmid & Vector & Reference/ Construction \\
\hline pGP1022 & pJOE6743 & modified pJOE (GeneArt) \\
\hline pGP1023 & pGP574/Sacl + BamHI & PCR-Prod.: citR/Sacl + BamHI with CZ238/CZ239 \\
\hline pGP1029 & pGP380/ BamHI + Pstl & \\
\hline pGP1030 & pGP382/ BamHI + Pstl & PCR-Prod.: citR/Pstl + BamHI with AK57/58 \\
& & PCR-Prod.: citR/PstI + BamHI with AK59/60 \\
\hline pGP2260 & pGP172/Sacl + BamHI & PCR-Prod.: citR/Sacl + BamHI with CZ224/CZ225 \\
\hline pGP2261 & pGP888/Kpnl + Xbal & PCR-Prod.: citZ/KpnI + Xbal with FM20/21 \\
\hline & &
\end{tabular}




\begin{tabular}{|c|c|c|}
\hline pGP2262 & pGP888/Kpnl + Xbal & PCR-Prod.: citR/KpnI + Xbal with AK89/90 \\
\hline pGP2263 & pGP1022/ BamHI + Ncol & $\begin{array}{l}\text { LFH-Prod.: yhfU for insertion between panB and } \\
\text { birA without promoters / BamHI +Ncol with } \\
\text { AK21/69 }\end{array}$ \\
\hline pGP2264 & pETM-11/ Ncol + BamHI & PCR-Prod.: citR/Ncol +BamHI with AK76/99 \\
\hline pGP2265 & pGP1022/ BamHI + Ncol & $\begin{array}{l}\text { LFH-Prod.: deletion yhfR-yhzC (bp 1.108.708- } \\
1.116 .817)\end{array}$ \\
\hline pGP2266 & pGP1022/ BamHI + Ncol & $\begin{array}{l}\text { LFH-Prod.: deletion } b g / C-y c g J(371,726-340,585) \\
\text { with integration of putP }\end{array}$ \\
\hline pGP2267 & pGP1022/ BamHI + Ncol & $\begin{array}{l}\text { LFH-Prod.: yhfU for insertion between panB and } \\
\text { birA without promotors, with long panB site / } \\
\text { BamHI +Ncol with AK21/69 }\end{array}$ \\
\hline pGP2268 & pBluescript II SK(-)/EcoRI + Xbal & $\begin{array}{l}\text { LFH-Prod.: ermR/EcoRI +Xbal, with AK101/ } \\
\text { AK102, lox sites added to the resistance with } \\
\text { primers }\end{array}$ \\
\hline pGP2269 & pGP1022/ BamHI + Xhol & $\begin{array}{l}\text { LFH-Prod. AK119/146 and AK147/148 for } \\
\text { deletion of ermR next to } y b g E\end{array}$ \\
\hline pGP2270 & pGP1022/ BamHI + Ncol & $\begin{array}{l}\text { LFH-Prod. AK206/207 and AK205/DR377 for the } \\
\text { deletion of alkA-ycbK }\end{array}$ \\
\hline pGP2271 & pGP1022/ BamHI + Ncol & $\begin{array}{l}\text { LFH-Prod. AK252/253 and AK254/AK255 for the } \\
\text { deletion of } y n f C \text {-iseA }\end{array}$ \\
\hline pGP2272 & pGP1022/ BamHI + Ncol & $\begin{array}{l}\text { LFH-Prod. AK258/259 and AK260/AK261 for the } \\
\text { deletion of pckA-mntA }\end{array}$ \\
\hline pGP2273 & $\mathrm{pAC5} / \mathrm{BamHI}+$ EcoRI & $\begin{array}{l}\text { PCR-Prod. promoter aapA/BamHI +EcoRI with } \\
\text { AK298/299 }\end{array}$ \\
\hline pGP2274 & $\mathrm{pAC5} / \mathrm{BamHI}+$ EcoRI & $\begin{array}{l}\text { PCR-Prod. promoter asnB/BamHI +EcoRI with } \\
\text { AK304/305 }\end{array}$ \\
\hline pGP2275 & $\mathrm{pAC5} / \mathrm{BamHI}+$ EcoRI & $\begin{array}{l}\text { PCR-Prod. promoter steT/BamHI +EcoRI with } \\
\text { AK300/301 }\end{array}$ \\
\hline pGP2276 & $\mathrm{pAC5} / \mathrm{BamHI}+$ EcoRI & $\begin{array}{l}\text { PCR-Prod. promoter ytnA/BamHI +ECoRI with } \\
\text { AK306/307 }\end{array}$ \\
\hline pGP2277 & $\mathrm{pAC5} / \mathrm{BamHI}+$ EcoRI & $\begin{array}{l}\text { PCR-Prod. promoter serA/BamHI +ECoRI with } \\
\text { AK308/309 }\end{array}$ \\
\hline pGP2278 & $\mathrm{pAC5} / \mathrm{BamHI}+$ EcoRI & $\begin{array}{l}\text { PCR-Prod. promoter yfnA/BamHI +EcoRI with } \\
\text { AK302/303 }\end{array}$ \\
\hline
\end{tabular}




\begin{tabular}{|c|c|c|}
\hline pGP2279 & pBQ200/BamHI + Sall & PCR-Prod. aapA/BamHI +Sall with AK240/241 \\
\hline pGP2280 & $\mathrm{pBQ} 200 /$ BamHI + Sall & PCR-Prod. ytnA/BamHI +Sall with AK246/247 \\
\hline pGP2281 & pBQ200/BamHI + Sall & PCR-Prod. yfnA/BamHI +Sall with AK244/245 \\
\hline pGP2282 & pGP1022/ BamHI + Ncol & $\begin{array}{l}\text { LFH-Prod. AK216/217 and AK215/DR393 for the } \\
\text { deletion of yqiF-yqiG }\end{array}$ \\
\hline pGP2283 & pGP1022/ BamHI + Ncol & $\begin{array}{l}\text { LFH-Prod. AK220/DR527 and AK219/DR387 for } \\
\text { the deletion of } y d d N-y d f M\end{array}$ \\
\hline pGP2284 & pGP1022/ BamHI + Ncol & $\begin{array}{l}\text { LFH-Prod. DR401/DR402 and AK222/AK223 for } \\
\text { the deletion of yqzK-yqjT }\end{array}$ \\
\hline pGP2285 & pBQ200/BamHI + Sall & PCR-Prod. steT/BamHI +Sall with AK242/243 \\
\hline pGP2286 & $\mathrm{pAC7} / \mathrm{BamHI}+\mathrm{EcoRI}$ & $\begin{array}{l}\text { PCR-Prod. promoter serA/BamHI +ECoRI with } \\
\text { AK308/309 }\end{array}$ \\
\hline pGP2287 & $\mathrm{pAC5} / \mathrm{BamHI}+$ EcoRI & $\begin{array}{l}\text { PCR-Prod. promoter ybeC/BamHI +EcoRI with } \\
\text { AK321/322 }\end{array}$ \\
\hline pGP2288 & pGP1022/ BamHI + Ncol & $\begin{array}{l}\text { LFH-Prod. AK313/314 and AK315/316 for the } \\
\text { deletion of } y q g M-p s t S\end{array}$ \\
\hline pGP2289 & pBQ200/BamHI + Sall & PCR-Prod. ilvA/BamHI +Sall with AK325/326 \\
\hline pGP2290 & pBQ200/BamHI + Sall & PCR-Prod. thrR/BamHI +Sall with AK333/DT126 \\
\hline pGP2291 & $\mathrm{pAC7} / \mathrm{BamHI}+$ EcoRI & $\begin{array}{l}\text { PCR-Prod. promoter thrR/BamHI +ECORI with } \\
\text { AK334/335 }\end{array}$ \\
\hline pGP2292 & pGP888/BamHI+EcoRI & PCR-Prod. ypkP/BamHI +EcoRI with AK360/361 \\
\hline pGP2293 & pGP888/BamHI+EcoRI & PCR-Prod. ypnP/BamHI +EcoRI with AK360/361 \\
\hline pGP2294 & $\mathrm{pAC5} / \mathrm{BamHI}+$ EcoRI & $\begin{array}{l}\text { PCR-Prod. promoter sdaAB mutated/BamHI } \\
+ \text { EcoRI with AK379/380 }\end{array}$ \\
\hline pGP2295 & $\mathrm{pAC5} / \mathrm{BamHI}+\mathrm{ECoRI}$ & $\begin{array}{l}\text { PCR-Prod. promoter sdaAB/BamHI +ECoRI with } \\
\text { AK379/380 }\end{array}$ \\
\hline pGP2296 & $\mathrm{pAC5} / \mathrm{BamHI}+\mathrm{ECORI}$ & $\begin{array}{l}\text { PCR-Prod. promoter hom mutated/BamHI +EcoRI } \\
\text { with } \mathrm{MT} 24 / 25\end{array}$ \\
\hline pGP2297 & pET-SUMOadapt/Eco31l + Xhol & PCR-Prod. hom/Eco31l + Xhol with AK349/350 \\
\hline
\end{tabular}




\begin{tabular}{lll}
\hline pGP2298 & pWH844/BamHI+ Sall & PCR-Prod. bcaP/BamHI + Sall with AK392/393 \\
\hline pGP2299 & pWH844/BamHI+Sall & PCR-Prod. ybxG /BamHI + Sall with AK394/395 \\
\hline pGP2300 & pWH844/BamHI+ Sacl & PCR-Prod. hom /BamHI + Sall with AK387/388
\end{tabular}

\subsubsection{Plasmids used in this work}

\begin{tabular}{|c|c|c|}
\hline Plasmid & Description & Reference \\
\hline pAC5 & $\begin{array}{l}\text { Construction of translational lacZ fusions in B. subtilis, } \\
\text { integrates into the amyE site }\end{array}$ & $\begin{array}{l}\text { Martin-Verstraete et al., } \\
1992\end{array}$ \\
\hline pAC7 & $\begin{array}{l}\text { Construction of translational lacZ fusions in B. subtilis, } \\
\text { integrates into the amyE site }\end{array}$ & Weinrauch et al., 1991 \\
\hline $\begin{array}{l}\text { pBlueskript } \\
\text { SK(-) }\end{array}$ & Vector for cloning & Stratagene \\
\hline pBQ200 & Constitutive overexpression of proteins in B. subtilis & $\begin{array}{l}\text { Martin-Verstraete et al., } \\
1992\end{array}$ \\
\hline pDG1726 & $\begin{array}{l}\text { Plasmid for the amplification of the spec cassette for } \\
\text { LFH-PCR }\end{array}$ & Guérout-Fleury et al., 1995 \\
\hline pDG780 & $\begin{array}{l}\text { Plasmid for the amplification of the kan cassette for } \\
\text { LFH-PCR }\end{array}$ & Guérout-Fleury et al., 1995 \\
\hline pETM-11 & $\begin{array}{l}\text { Overexpression of C-terminal His-Tag fusion proteins in } \\
\text { E. coli; the His-Tag can be cleaved off with the TEV } \\
\text { Protease }\end{array}$ & Dümmler et al., 2005 \\
\hline $\begin{array}{l}\text { pET-SUMO } \\
\text { adapt }\end{array}$ & $\begin{array}{l}\text { Fusion of SUMO protein and a His(6) tag at the } \mathrm{N} \text { - } \\
\text { terminus of a protein for inducible overexpression via } \\
\text { IPTG in E. coli }\end{array}$ & Hanington et al., 2006 \\
\hline pGEM-cm & $\begin{array}{l}\text { Plasmid for the amplification of the cat cassette for LFH- } \\
\text { PCR }\end{array}$ & Guérout-Fleury et al., 1995 \\
\hline pGP172 & $\begin{array}{l}\text { Fusion of Strep-tag at the N-terminus of a protein for } \\
\text { inducible overexpression via IPTG in E. coli }\end{array}$ & Merzbacher 2004 \\
\hline pGP2072 & $\begin{array}{l}\text { LFH-Prod. DR337/DR338 and DR339/DR340 for deletion } \\
\text { of yobQ-desR }\end{array}$ & Reuß, 2017 \\
\hline pGP2073 & $\begin{array}{l}\text { LFH-Prod. DR343/DR344 and DR345/DR346 for deletion } \\
\text { of nhaX-yhaX }\end{array}$ & Reuß, 2017 \\
\hline
\end{tabular}




\begin{tabular}{|c|c|c|}
\hline pGP2093 & $\begin{array}{l}\text { LFH-Prod. DR525/DR526 and DR527/DR528 for deletion } \\
\text { of } m h q N-m h q P\end{array}$ & Reuß, 2017 \\
\hline pGP2094 & $\begin{array}{l}\text { LFH-Prod. DR531/DR532 and DR533/DR534 for deletion } \\
\text { of yuzG-sufA }\end{array}$ & Reuß, 2017 \\
\hline pGP2514 & $\begin{array}{l}\text { Plasmid for amplification of kan-lox for the cre-lox } \\
\text { system }\end{array}$ & Zschiedrich, 2014 \\
\hline pGP2515 & $\begin{array}{l}\text { N-terminal Strep-tag Fusion to CitZ for IPTG induced } \\
\text { overexpression in E. coli }\end{array}$ & Zschiedrich, 2014 \\
\hline pGP2516 & $\begin{array}{l}\mathrm{N} \text {-terminal Strep-tag Fusion to CitA for IPTG induced } \\
\text { overexpression in E. coli }\end{array}$ & Zschiedrich, 2014 \\
\hline pGP2980 & pGP886 with ybeC & Larissa Krüger, $\mathrm{PhD}$ thesis \\
\hline pGP2987 & pWH844 with ybeC & Larissa Krüger, $\mathrm{PhD}$ thesis \\
\hline pGP2988 & pWH844 with ybeC without C-terminus & Larissa Krüger, PhD thesis \\
\hline pGP380 & $\begin{array}{l}\text { Expression of proteins in B. subtilis allows fusion to a } \\
\text { Strep-tag at the N-terminus of the protein }\end{array}$ & Herzberg et al., 2007 \\
\hline pGP382 & $\begin{array}{l}\text { Expression of proteins in B. subtilis allows fusion to a } \\
\text { Strep-tag at the C-terminus of the protein }\end{array}$ & Herzberg et al., 2007 \\
\hline pGP574 & $\begin{array}{l}\text { Fusion of Strep-tag at the C-terminus of a protein for } \\
\text { inducible overexpression via IPTG in E. coli }\end{array}$ & Schilling et al., 2006 \\
\hline pGP886 & $\begin{array}{l}\text { Integration vector (integrates in } y k d E \text { ); allowing the } \\
\text { expression of genes under the control of the xylose- } \\
\text { inducible PxylA promoter in B. subtilis }\end{array}$ & Gerwig et al., 2014 \\
\hline pGP888 & $\begin{array}{l}\text { Integration vector (integrates in ganA); allowing the } \\
\text { expression of genes under the control of the xylose- } \\
\text { inducible PxylA promoter in B. subtilis }\end{array}$ & Diethmaier et al., 2011 \\
\hline pJK195 & $\begin{array}{l}\text { Integration of the xylose, inducible cre recombinase in } \\
\text { the sacA locus }\end{array}$ & Kumpfmüller et al., 2013 \\
\hline pJOE6743.1 & Marker-free deletion in a manPA deletion strain & $\begin{array}{l}\text { Wenzel and Altenbuchner, } \\
2015\end{array}$ \\
\hline pJOE6981.2 & $\begin{array}{l}\text { Plasmid for the marker-free deletion of spollIAH-yqhV } \\
(2,532,960-2,538,080)\end{array}$ & $\begin{array}{l}\text { Josef Altenbuchner, } \\
\text { Stuttgart }\end{array}$ \\
\hline pJOE8670.1 & $\begin{array}{l}\text { Plasmid for the marker-free deletion of mta-rapB } \\
(3,764,119-3,772,301)\end{array}$ & $\begin{array}{l}\text { Josef Altenbuchner, } \\
\text { Stuttgart }\end{array}$ \\
\hline
\end{tabular}




\begin{tabular}{lll}
\hline pJOE9255 & pJOE6743 via BamHI, deletes ypsA-sspM & Josef Altenbuchner, \\
& & Stuttgart \\
\hline pJOE9256 & pJOE6743 via BamHI, deletes uxaC-spollSA & Josef Altenbuchner, \\
& & Stuttgart \\
\hline pWH844 & Fusion of His(6) tag at the N-terminus of a protein for & Schirmer et al., 1997 \\
& inducible overexpression via IPTG in E. coli & \\
\hline
\end{tabular}

\subsection{Oligonucleotides}

\subsubsection{Oligonucleotides constructed in this work}

\begin{tabular}{|c|c|c|}
\hline Name & Sequence & Purpose/ Reference \\
\hline AK18 & [phos]CCATCAAAAACCGGTCTGCCATACG & fwd, yhfU for LCR \\
\hline AK19 & [phos]TCATCCTCCTTTTGTAAACATCGTATCAGAAAG & rev, yhfU for LCR \\
\hline AK20 & [phos]TGTGTTGGTACAAGCCCGTTGATTTTG & $\begin{array}{l}\text { fwd, upstream LCR of yhfU, in } \\
\text { panB }\end{array}$ \\
\hline AK21 & [phos]TTTGGATCCACTTTATAGCCGCCCAGTACGCCG & rev, upstream LCR of yhfU \\
\hline AK22 & [phos]AAAGGATCCAAGCACGCAAAAAACGGCTCATGAGC & $\begin{array}{l}\text { fwd, downstream LCR of } y h f U \text {, } \\
\text { in birA }\end{array}$ \\
\hline AK23 & [phos] TTAGCCCAATTCGATATCGGCAGAATAG & $\begin{array}{l}\text { rev, downstream LCR of } y h f U \text {, in } \\
\text { birA }\end{array}$ \\
\hline AK24 & $\begin{array}{l}\text { CTATTCTGCCGATATCGAATTGGGCTAACCATCAAAAACCG } \\
\text { GTCTGCCATACG }\end{array}$ & $\begin{array}{l}\text { bridging oligo LCR of upstream } \\
+y h f U\end{array}$ \\
\hline AK25 & $\begin{array}{l}\text { CTTTCTGATACGATGTTTACAAAAGGAGGATGATGTGTTGG } \\
\text { TACAAGCCCGTTGATTTTG }\end{array}$ & $\begin{array}{l}\text { bridging oligo LCR of yhfU + } \\
\text { downstream }\end{array}$ \\
\hline AK26 & AAAGGATCCGATCCATAACGGAATGCTGAACCAGAC & $\begin{array}{l}\text { fwd, downstream LCR of } \\
\text { menaquinone, in } m n t A\end{array}$ \\
\hline AK27 & $\begin{array}{l}\text { [phos]GAAAAAAGCATTTTGCTATTTTGAATAAATGACACT } \\
\text { G }\end{array}$ & $\begin{array}{l}\text { rev, downstream LCR of } \\
\text { menaquinone, in } m n t A\end{array}$ \\
\hline AK28 & [phos] TTAACCATGCTGTGTGAAAACATCCATTTTGG & $\begin{array}{l}\text { fwd, upstream LCR of } \\
\text { menaquinone, in menC }\end{array}$ \\
\hline AK29 & TTTGAATTCCCTGGCGCCTCTTGACGATATG & $\begin{array}{l}\text { rev, upstream LCR of } \\
\text { menaquinone, upstream of } \\
\text { menC }\end{array}$ \\
\hline
\end{tabular}




\begin{tabular}{|c|c|c|}
\hline AK30 & [phos] TTATCGGAAATAGCTGATCAATAATCCGATC & fwd, menA or LCR \\
\hline AK31 & [phos] ATCAAAAATTCCCTTCCCGTTTTTTTCGACAATC & rev, menA for LCR, upstream \\
\hline AK32 & [phos]TTAAAATTTTCTTTTACCGATATATTTTGCGATGGC & fwd, hepT-menH-hepS for LCR \\
\hline AK33 & [phos] GCTGGCTGTCCCCGCTGTTAAAA & $\begin{array}{l}\text { rev, hepT-menH-hepS for LCR, } \\
\text { upstream }\end{array}$ \\
\hline AK34 & $\begin{array}{l}\text { CAGTGTCATTTATTCAAAATAGCAAAATGCTTTTTTCTTATC } \\
\text { GGAAATAGCTGATCAATAATCCGATC }\end{array}$ & $\begin{array}{l}\text { briging oligo for the LCR of } \\
\text { downstream+ menA }\end{array}$ \\
\hline AK35 & $\begin{array}{l}\text { GATTGTCGAAAAAACGGGAAGGGAATTTTTGATTTAAAATT } \\
\text { TTCTTTTACCGATATATTTTGCGATGGC }\end{array}$ & $\begin{array}{l}\text { briging oligo for the LCR of } \\
\text { menA+ hepT-menH-hepS }\end{array}$ \\
\hline AK36 & $\begin{array}{l}\text { TTTTAACAGCGGGGACAGCCAGCTTAACCATGCTGTGTGAA } \\
\text { AACATCCATTTTGG }\end{array}$ & $\begin{array}{l}\text { briging oligo for the LCR of } \\
\text { hepT-menH-hepS+ upstream }\end{array}$ \\
\hline AK37 & [phos] CTTGCCTATGGATACAGATCGCG & Fwd, CCR primer menA \\
\hline AK38 & [phos] CGCGATCTGTATCCATAGGCAAG & Rev, CCR primer menA \\
\hline AK39 & $\begin{array}{l}\text { AAAGTCGACCGCTTGTAAACCGTTTTGTGAAAAAATTTTTA } \\
\text { AAATAAAAAAG }\end{array}$ & $\begin{array}{l}\text { amplification of pJOE6743 from } \\
\text { the CDS ter }\end{array}$ \\
\hline AK40 & $\begin{array}{l}\text { TTTGTCGACCTTGTAAACCGTTTTGTGAAAAAATTTTTAAAAA } \\
\text { TAAAAAAGGGG }\end{array}$ & $\begin{array}{l}\text { rev CDS ter, construction of } \\
\text { modif. pJOE }\end{array}$ \\
\hline AK41 & [phos] CATCCGGTGGATGACCTTTTGAATGAC & $\begin{array}{l}\text { fwd CDS ter, construction of } \\
\text { modif. pJOE }\end{array}$ \\
\hline AK42 & [phos] CAATACGCAAACCGCCTCTCCC & $\begin{array}{l}\text { rev Insert pJOE, construction of } \\
\text { modif. pJOE }\end{array}$ \\
\hline AK43 & [phos] TCTAGAGGATCCCCGGGTACCAT & $\begin{array}{l}\text { fwd MCS of pJOE6743, } \\
\text { construction of modif. pJOE }\end{array}$ \\
\hline AK44 & $\begin{array}{l}\text { [phos]GTGACACTATAGAAGATCGATGAATTCGAGCTCGT } \\
\text { ACGC }\end{array}$ & $\begin{array}{l}\text { rev MCS of pJOE6743, } \\
\text { construction of modif. pJOE }\end{array}$ \\
\hline AK45 & $\begin{array}{l}\text { [phos]CTAAATCGTATGCCATCCGGTGGATGACCTTTTGAA } \\
\text { TGAC }\end{array}$ & $\begin{array}{l}\text { fwd CDS ter, construction of } \\
\text { modif. pJOE }\end{array}$ \\
\hline AK46 & $\begin{array}{l}\text { GTCATTCAAAAGGTCATCCACCGGATGGTAAAGCTTGCATG } \\
\text { CCTAATACGAC }\end{array}$ & $\begin{array}{l}\text { bridging oligo for the LCR of } \\
\text { CDS term +DR207 fragment }\end{array}$ \\
\hline AK47 & $\begin{array}{l}\text { GAGAGGCGGTTTGCGTATTGTCTAGAGGATCCCCGGGTAC } \\
\text { C }\end{array}$ & $\begin{array}{l}\text { bridging oligo for the LCR of } \\
\text { DR207 fragment + MCS }\end{array}$ \\
\hline
\end{tabular}




\begin{tabular}{|c|c|c|}
\hline AK48 & $\begin{array}{l}\text { CGAATTCATCGATCTTCTATAGTGTCACCTAAATCGTATGCC } \\
\text { ATCCGGTGGATGAC }\end{array}$ & $\begin{array}{l}\text { bridging oligo for the LCR of } \\
\mathrm{MCS}+\mathrm{CDS} \text { term }\end{array}$ \\
\hline AK49 & [phos] GTAAAGCTTGCATGCCTAATACGAC & DR207 with phosphorylation \\
\hline AK50 & CAGTTGCGCAGCCTGAATGGC & Sequencing primer modif. pJOE \\
\hline AK51 & CGCGGGGAGAGGCGGTTTG & Sequencing primer modif. pJOE \\
\hline AK52 & CTCTTCGCTATTACGCCAATCTAGATCC & Sequencing primer pGP1022 \\
\hline AK53 & GTTGGCCGATTCATTAATGCAGATCGATC & Sequencing primer pGP1022 \\
\hline AK54 & AAAGCTAGCGTCAACATACGTAGAAGTTGTGATCTCTCC & $\begin{array}{l}\text { fwd, downstream, LCR of } \\
\text { menaquinone, in metK }\end{array}$ \\
\hline AK55 & [phos] CAACTGAGTTCATATGAAACCTTCCTTTATCG & $\begin{array}{l}\text { rev, downstream LCR of } \\
\text { menaquinone, in metK }\end{array}$ \\
\hline AK56 & $\begin{array}{l}\text { CGATAAAGGAAGGTTTCATATGAACTCAGTTGTTATCGGAA } \\
\text { ATAGCTGATCAATAATCCGATC }\end{array}$ & $\begin{array}{l}\text { bridging oligo for the LCR of } \\
\text { metK+ menA }\end{array}$ \\
\hline AK57 & AAAGGATCCATGGATTTCAAATGGCTTCACACCTTTG & $\begin{array}{l}\text { fwd, citR for N-term. Strep-tag } \\
\text { fusion in pGP380 }\end{array}$ \\
\hline AK58 & $\begin{array}{l}\text { TTTCTGCAGCTCCTAAAAATGAAAATGTGATAAAAAATCCA } \\
\text { AGAAC }\end{array}$ & $\begin{array}{l}\text { rev, citR for } \mathrm{N} \text {-term. Strep-tag } \\
\text { fusion in } \mathrm{pGP380}\end{array}$ \\
\hline AK59 & AAAGGATCCCAGAGGGAGAATAGAAATGGATTTCAAATG & $\begin{array}{l}\text { fwd, citR for C-term. Strep-tag } \\
\text { fusion in pGP382 }\end{array}$ \\
\hline AK60 & $\begin{array}{l}\text { TTTCTGCAGAAAATGAAAATGTGATAAAAAATCCAAGAACT } \\
\text { TTTTTTCTTTC }\end{array}$ & $\begin{array}{l}\text { rev, citR for C-term. Strep-tag } \\
\text { fusion in pGP382 }\end{array}$ \\
\hline AK61 & AAAGAATCCCCCCAATGCCTTTTTTATAGTATATG & $\begin{array}{l}\text { fwd, upstream (comK) of yhfU } \\
\text { deletion region }\end{array}$ \\
\hline AK62 & AGAGATCGGCAGCTCCATCGTTTTC & $\begin{array}{l}\text { rev, upstream (comK) of yhfU } \\
\text { deletion region }\end{array}$ \\
\hline AK63 & CGGCTACACATCTGTCGGCAC & $\begin{array}{l}\text { fwd, downstream }(y h f Q) \text { of } \\
y h f U \text { deletion region }\end{array}$ \\
\hline AK64 & $\begin{array}{l}\text { CATATACTATAAAAAAGGCATTGGGGGGATTCTTT } \\
\text { CTTTTATTTCTTAGCAGCCGGCATCTCTTTTTG }\end{array}$ & $\begin{array}{l}\text { rev, downstream ( } y h f Q) \text { of } y h f U \\
\text { deletion region }\end{array}$ \\
\hline AK65 & $\begin{array}{l}\text { AAAGGTCTCATGGTATGGATTTCAAATGGCTTCACACCTTT } \\
\text { GTG }\end{array}$ & fwd, citR for pET-SUMOadapt \\
\hline
\end{tabular}




\begin{tabular}{|c|c|c|}
\hline AK66 & $\begin{array}{l}\text { TTTCTCGAGCTAAAAATGAAAATGTGATAAAAAATCCAAGA } \\
\text { ACTTTTTTTC }\end{array}$ & rev, citR for pET-SUMOadapt \\
\hline AK67 & $\begin{array}{l}\text { CGTATGGCAGACCGGTTTTTGATGGTTAGCCCAATTCGATA } \\
\text { TCGGCAGAATAG }\end{array}$ & $\begin{array}{l}\text { bridging oligo LCR of upstream } \\
+y h f U\end{array}$ \\
\hline AK68 & $\begin{array}{l}\text { CAAAATCAACGGGCTTGTACCAACACATCATCCTCCTTTTGT } \\
\text { AAACATCGTATCAGAAAG }\end{array}$ & $\begin{array}{l}\text { bridging oligo LCR of yhfU + } \\
\text { downstream }\end{array}$ \\
\hline AK69 & TTTCCATGGAAGCACGCAAAAAACGGCTCATGAGC & $\begin{array}{l}\text { fwd, downstream, LCR of yhfU, } \\
\text { in birA }\end{array}$ \\
\hline AK70 & $\begin{array}{l}\text { GTATAATGTATGCTATACGAACGGTACTGAATAAATCAGT } \\
\text { TGAATCTGTC }\end{array}$ & $\begin{array}{l}\text { rev, upstream LFH of citZ-icd- } \\
\text { mdh }\end{array}$ \\
\hline AK71 & $\begin{array}{l}\text { GTATAGCATACATTATACGAACGGTAGTTGTTGCTACAAC } \\
\text { CССTTC }\end{array}$ & $\begin{array}{l}\text { fwd, downstream LFH of citZ- } \\
\text { icd-mdh }\end{array}$ \\
\hline AK72 & $\begin{array}{l}\text { CCTATCACCTCAAATGGTTCGCTGCTAAAAATGAAAATGTG } \\
\text { ATAAAAAATCCA }\end{array}$ & $\begin{array}{l}\text { citR reverse, fusion of lacA-cat- } \\
\text { citR-lacA }\end{array}$ \\
\hline AK73 & $\begin{array}{l}\text { CAGCCATATTGATGGTGAAAAAGCCGTATCGTTCTGCTAA } \\
\text { TAAGC }\end{array}$ & $\begin{array}{l}\text { lacA reverse downstream, } \\
\text { fusion of IacA-cat-citR-lacA }\end{array}$ \\
\hline AK74 & $\begin{array}{l}\text { CTTTCTGATACGATGTTTACAAAAGGAGGATGACATGAAA } \\
\text { ACAAAACTGGATTTTCTAAAAATGAAGGA }\end{array}$ & $\begin{array}{l}\text { downstream fwd panB, yhfU } \\
\text { LFH without promoter }\end{array}$ \\
\hline AK75 & $\begin{array}{l}\text { CTATTCTGCCGATATCGAATTGGGCTAAATGCTGAAATTAA } \\
\text { TCGACATGATGCATATTGCG }\end{array}$ & $\begin{array}{l}\text { fwd, yhfU for LFH, without } \\
\text { promoter }\end{array}$ \\
\hline AK76 & AAACCATGGAAATGGATTTCAAATGGCTTCACACCTTTGTG & $\begin{array}{l}\text { fwd, citR for N-terminal His-tag } \\
\text { fusion in } \mathrm{pETM-11}\end{array}$ \\
\hline AK77 & $\begin{array}{l}\text { GTATAATGTATGCTATACGAACGGTACTATACCTGAATCTT } \\
\text { CTAACGC }\end{array}$ & rev, upstream LFH citB \\
\hline AK78 & $\begin{array}{l}\text { GTATAGCATACATTATACGAACGGTACATCCTTCAAATGGT } \\
\text { GCTTCG }\end{array}$ & fwd, downstream LFH citB \\
\hline AK79 & $\begin{array}{l}\text { GTATAGCATACATTATACGAACGGTACTGGAAGATCCTGA } \\
\text { ACAGCT }\end{array}$ & fwd, downstream LFH odhAB \\
\hline AK80 & $\begin{array}{l}\text { GTATAATGTATGCTATACGAACGGTAGTAAAAATCTTCCCA } \\
\text { ATTCATTC }\end{array}$ & rev, upstream LFH odhAB \\
\hline AK81 & $\begin{array}{l}\text { GTATAATGTATGCTATACGAACGGTACACTTTACCTTCAGG } \\
\text { AACAGATACCCC }\end{array}$ & rev, upstream LFH sucCD \\
\hline AK82 & $\begin{array}{l}\text { GTATAGCATACATTATACGAACGGTAGCAGAGACACCTTC } \\
\text { TGTCATGGGTG }\end{array}$ & fwd, downstream LFH sucCD \\
\hline AK83 & $\begin{array}{l}\text { GTATAATGTATGCTATACGAACGGTAGACGACTAAATGCT } \\
\text { GAATAAG }\end{array}$ & rev, upstream LFH sdhCAB \\
\hline
\end{tabular}




\begin{tabular}{|c|c|c|}
\hline AK84 & $\begin{array}{l}\text { GTATAGCATACATTATACGAACGGTAGATTGCAGCCTTGA } \\
\text { ATAGAG }\end{array}$ & fwd, downstream LFH sdhCAB \\
\hline AK85 & $\begin{array}{l}\text { GTATAGCATACATTATACGAACGGTACGGAAGACATGGTA } \\
\text { AAACCAAAGGCG }\end{array}$ & downstream LFH citG \\
\hline AK86 & $\begin{array}{l}\text { GTATAATGTATGCTATACGAACGGTACCATGGTGTCTCGTT } \\
\text { CAATTCTGTATTCC }\end{array}$ & rev, upstream LFH citG \\
\hline AK87 & $\begin{array}{l}\text { CCTATCACCTCAAATGGTTCGCTGGAGCACAGGCGTTTTGG } \\
\text { TTGCTCC }\end{array}$ & $\begin{array}{l}\text { rev, citR for the integration into } \\
\text { lacA (lacA-cat-citR-fusion) }\end{array}$ \\
\hline AK88 & CGTAATGTACCATTTGTATTCCCCCTATCC & $\begin{array}{l}\text { fwd, citR for the integration } \\
\text { into lacA (lacA-cat-citR-fusion) }\end{array}$ \\
\hline AK89 & AAATCTAGAAATGGATTTCAAATGGCTTCACACCTTTG & $\begin{array}{l}\text { fwd, citR for the integration } \\
\text { into lacA with pGP888 }\end{array}$ \\
\hline AK90 & $\begin{array}{l}\text { TTTGGTACCCTCCTAAAAATGAAAATGTGATAAAAAATCCA } \\
\text { AGAAC }\end{array}$ & $\begin{array}{l}\text { rev, citR for the integration into } \\
\text { lacA with pGP888 }\end{array}$ \\
\hline AK91 & $\begin{array}{l}\text { GATCGGATTATTGATCAGCTATTTCCGATAACAACTGAGTT } \\
\text { CATATGAAACCTTCCTTTATCG }\end{array}$ & $\begin{array}{l}\text { rev, downstream, LCR of menA, } \\
\text { in metK }\end{array}$ \\
\hline AK92 & $\begin{array}{l}\text { GATTGTCGAAAAAACGGGAAGGGAATTTTTTATTTAACCA } \\
\text { TGCTGTGTGAAAACATCCATTTTGG }\end{array}$ & $\begin{array}{l}\text { fwd, upstream, LCR of menA, in } \\
\text { menC }\end{array}$ \\
\hline AK93 & $\begin{array}{l}\text { CGATAAAGGAAGGTTTCATATGAACTCAGTTGTTATCGGA } \\
\text { AATAGCTGATCAATAATCCGATC }\end{array}$ & fwd, menA for LCR \\
\hline AK94 & $\begin{array}{l}\text { CCAAAATGGATGTTTTCACACAGCATGGTTAAATCAAAAA } \\
\text { TTCCСTTCCCGTTTTTTCGACAATC }\end{array}$ & $\begin{array}{l}\text { rev, menA for LCR, binds } \\
\text { upstream }\end{array}$ \\
\hline AK95 & AAAGAATCCCCCCAATGCCTTTTTTATAGTATATG & $\begin{array}{l}\text { rev, upstream yhfU deletion } \\
\text { region }\end{array}$ \\
\hline AK96 & AAAGGATCCAGAGATCGGCAGCTCCATCGTTTTC & $\begin{array}{l}\text { fwd, upstream yhfU deletion } \\
\text { region }\end{array}$ \\
\hline AK97 & TTTCCATGGCGGCTACACATCTGTCGGCAC & $\begin{array}{l}\text { rev, downstream yhfU deletion } \\
\text { region }\end{array}$ \\
\hline AK98 & $\begin{array}{l}\text { CATATACTATAAAAAAGGCATTGGGGGGATTCTTTCTTTTA } \\
\text { TTTCTTAGCAGCCGGCATCTCTTTTTG }\end{array}$ & $\begin{array}{l}\text { fwd, downstream yhfU deletion } \\
\text { region }\end{array}$ \\
\hline AK99 & $\begin{array}{l}\text { TTTGGATCCCTAAAAATGAAAATGTGATAAAAAATCCAAGA } \\
\text { ACTTTTTTTC }\end{array}$ & $\begin{array}{l}\text { rev, citR for } \mathrm{N} \text {-terminal His-tag } \\
\text { fusion in } \mathrm{pETM}-11\end{array}$ \\
\hline AK100 & AAAGGATCCCGATGGTTTCATCAATGCGCGTATATTGC & $\begin{array}{l}\text { rev, upstream, LCR of } y h f U \\
\text { (long) }\end{array}$ \\
\hline AK101 & $\begin{array}{l}\text { AAAGAATTCTACCGTTCGTATAGCATACATTATACGAAGTT } \\
\text { ATGATCCTTTAACTCTGGCAACCCTCAAAATTG }\end{array}$ & $\begin{array}{l}\text { fwd, erm resistance +lox71 for } \\
\text { pBluescript }\end{array}$ \\
\hline
\end{tabular}




\begin{tabular}{|c|c|c|}
\hline AK102 & $\begin{array}{l}\text { TTTTCTAGATACCGTTCGTATAATGTATGCTATACGAAGTTA } \\
\text { TGCCGACTGCGCAAAAGACATAATCG }\end{array}$ & $\begin{array}{l}\text { rev, erm resistance +lox71 for } \\
\text { pBluescript }\end{array}$ \\
\hline AK103 & $\begin{array}{l}\text { AAAGAATTCTACCGTTCGTATAGCATACATTATACGAAGTT } \\
\text { ATCGGCAATAGTTACCCTTATTATCAAGATAAGAAAG }\end{array}$ & $\begin{array}{l}\text { fwd, cat resistance +lox71 for } \\
\text { pBluescript }\end{array}$ \\
\hline AK104 & $\begin{array}{l}\text { TTTTCTAGATACCGTTCGTATAATGTATGCTATACGAAGTTA } \\
\text { TCCAGCGTGGACCGGCGAG }\end{array}$ & $\begin{array}{l}\text { rev, cat resistance +lox71 for } \\
\text { pBluescript }\end{array}$ \\
\hline AK105 & AAAGGATCCGCCAAGCATATTAAGGATGCGATAAATGAG & $\begin{array}{l}\text { fwd, downstream, } \\
\text { defragmentation of putP (LFH) }\end{array}$ \\
\hline AK106 & $\begin{array}{l}\text { CGACTACCAAGATACAATGTCGCAATAAAGCATTACACAG } \\
\text { AAACAGCATCAATAATATAAGTGTC }\end{array}$ & $\begin{array}{l}\text { rev, downstream, } \\
\text { defragmentation of putP (LFH) }\end{array}$ \\
\hline AK107 & $\begin{array}{l}\text { GAGGATGCCCATTTAGTCCCGCTTACATCGGCTGATAAAG } \\
\text { ATCCCAGC }\end{array}$ & $\begin{array}{l}\text { fwd, upstream, } \\
\text { defragmentation of putP (LFH) }\end{array}$ \\
\hline AK108 & AAACCATGGGTACAATGAATGTCATTCAGGCAAAAATGGC & $\begin{array}{l}\text { rev, upstream, defragmentation } \\
\text { of putP (LFH) }\end{array}$ \\
\hline AK109 & CTTTATTGCGACATTGTATCTTGGTAGTCG & $\begin{array}{l}\text { fwd, putP defragmentation of } \\
\text { putP (LFH) }\end{array}$ \\
\hline AK110 & GCGGGACTAAATGGGCATCCTC & $\begin{array}{l}\text { rev, putP defragmentation of } \\
\text { putP (LFH) }\end{array}$ \\
\hline AK111 & CTTCAACCGCAACTGTACAGGTGC & $\begin{array}{l}\text { rev, putP defragmentation of } \\
\text { putP (LFH) }\end{array}$ \\
\hline AK112 & $\begin{array}{l}\text { GCACCTGTACAGTTGCGGTTGAAGTTACATCGGCTGATAA } \\
\text { AGATCCCAGC }\end{array}$ & $\begin{array}{l}\text { fwd, upstream, } \\
\text { defragmentation putP (LFH) }\end{array}$ \\
\hline AK113 & AAAGGATCCGCTGTTTTGCTTATGCAAAACAGCTTTTTTGTC & $\begin{array}{l}\text { fwd, upstream, } \\
\text { defragmentation tcyP (LFH) }\end{array}$ \\
\hline AK114 & GTGACCATACTCCTATCTATGTATTAGAGCATG & $\begin{array}{l}\text { rev, upstream, defragmentation } \\
\operatorname{tcy} P(\mathrm{LFH})\end{array}$ \\
\hline AK115 & $\begin{array}{l}\text { CATGCTCTAATACATAGATAGGAGTATGGTCACGGTCAAA } \\
\text { TTTACAAACAGTTCTTTCAGCAAATATTATC }\end{array}$ & $\begin{array}{l}\text { fwd, tcyP, defragmentation tcyP } \\
\text { (LFH) }\end{array}$ \\
\hline AK116 & $\begin{array}{l}\text { GGGCTGCAGACAGCCCGTTATGATGTTACGCTTCTTCAGCT } \\
\text { TCAATCACTCTG }\end{array}$ & $\begin{array}{l}\text { rev, tcy } P \text {, defragmentation tcy } P \\
(\mathrm{LFH})\end{array}$ \\
\hline AK117 & ATCATAACGGGCTGTCTGCAGCCC & $\begin{array}{l}\text { fwd, downstream, } \\
\text { defragmentation tcy } P \text { (LFH) }\end{array}$ \\
\hline AK118 & TTTCCATGGCTTCGTCCACTGTGATCGTCAGCT & $\begin{array}{l}\text { rev, downstream, } \\
\text { defragmentation tcyP (LFH) }\end{array}$ \\
\hline AK119 & AAAGGATCCCCTGACAATCACAAACGTACCTGGATC & $\begin{array}{l}\text { fwd, upstream, } \\
\text { defragmentation ybgE (LFH) }\end{array}$ \\
\hline
\end{tabular}




\begin{tabular}{|c|c|c|}
\hline AK120 & $\begin{array}{l}\text { AATTGATCTTCCGCGCCCATGCAAACTTACTCCACAGTAAC } \\
\text { ACTCTTCGCAAGGTT }\end{array}$ & $\begin{array}{l}\text { rev, upstream, defragmentation } \\
y b g E(\mathrm{LFH})\end{array}$ \\
\hline AK121 & GTTTGCATGGGCGCGGAAGATCAATT & $\begin{array}{l}\text { fwd, ybgE, defragmentation } \\
y b g E(\mathrm{LFH})\end{array}$ \\
\hline AK122 & $\begin{array}{l}\text { GCCGGAACACCATTTCGTCATCAAGTCACACTTCCACTGTC } \\
\text { CAGTTAAACGG }\end{array}$ & $\begin{array}{l}\text { rev, ybgE, defragmentation } \\
y b g E(\mathrm{LFH})\end{array}$ \\
\hline AK123 & CTTGATGACGAAATGGTGTTCCGGC & $\begin{array}{l}\text { fwd, downstream, } \\
\text { defragmentation ybgE (LFH) }\end{array}$ \\
\hline AK124 & TTTCTCGAGCCATACGTGAGGGTCCATTGCATGA & $\begin{array}{l}\text { rev, downstream, } \\
\text { defragmentation ybgE (LFH) }\end{array}$ \\
\hline AK125 & AAAGGATCCCCGTCAGTGAAACGGTAGGTTTTCC & $\begin{array}{l}\text { fwd, downstream, } \\
\text { defragmentation } m n t H(L F H)\end{array}$ \\
\hline AK126 & CACGGGTGGTCGGTTATTGATTAAGTTTC & $\begin{array}{l}\text { rev, downstream, } \\
\text { defragmentation } m n t H(L F H)\end{array}$ \\
\hline AK127 & $\begin{array}{l}\text { GAAACTTAATCAATAACCGACCACCCGTGTTATCGAAACG } \\
\text { TATCTACAATTAAAAACACATTCAAAGC }\end{array}$ & $\begin{array}{l}\text { fwd, } m n t H \text {, defragmentation } \\
m n t H(\text { LFH) }\end{array}$ \\
\hline AK128 & CATTTTCGGTTGACAAGAAACCGGGATG & $\begin{array}{l}\text { rev, } m n t H \text {, defragmentation } \\
m n t H(\mathrm{LFH})\end{array}$ \\
\hline AK129 & $\begin{array}{l}\text { CATCCCGGTTTCTTGTCAACCGAAAATGCGGCCATTATCGG } \\
\text { GCTGAAACAATTTCGT }\end{array}$ & $\begin{array}{l}\text { fwd, upstream, } \\
\text { defragmentation } m n t H(L F H)\end{array}$ \\
\hline AK130 & TTTCTCGAGAGCGCCAATGCCAAAACGCCTGAA & $\begin{array}{l}\text { rev, upstream, defragmentation } \\
m n t H(\mathrm{LFH})\end{array}$ \\
\hline AK131 & CAGCCTGACAAAAGCAATACCTATGTCG & fwd, sequencing of yveA \\
\hline AK132 & CTTTATTTATCATAATCAATGGCGGCTTTTGGG & rev, sequencing of yveA \\
\hline AK133 & GCAATGAGAACTCCCGGCCAATTG & rev, sequencing of yveA \\
\hline AK134 & CTATGAGCACACACAAAAAGAGCCCAC & fwd, sequencing of $y \operatorname{tn} A$ \\
\hline AK135 & CTTGCGGATCTCTGGCGAATGCT & fwd, sequencing of yodF \\
\hline AK136 & CTGCCGCGTATGGTTTTTTTATAGCGC & rev, sequencing of yodF \\
\hline AK137 & GTCACACGTTCAATGGTGTTTGTTGTCATC & fwd, sequencing of yod $F$ \\
\hline AK138 & CTCGAGGCCGTGTCAGTGATGAAGGGCC & $\begin{array}{l}\text { rev, LFH deletion plasmid for } \\
m n t H \text { defragmentation }\end{array}$ \\
\hline
\end{tabular}




\begin{tabular}{|c|c|c|}
\hline AK139 & $\begin{array}{l}\text { GAGGGTTGCCAGAGTTAAAGGATCCACGGGTGGTCGGTT } \\
\text { ATTGATTAAGTTTC }\end{array}$ & $\begin{array}{l}\text { fwd, downstream, LFH for } m n t H \\
\text { defragmentation }\end{array}$ \\
\hline AK140 & $\begin{array}{l}\text { CGATTATGTCTTTTGCGCAGTCGGCTTATCGAAACGTATCT } \\
\text { ACAATTAAAAACACATTCAAAGC }\end{array}$ & $\begin{array}{l}\text { rev, } m n t H, \text { LFH for } m n t H \\
\text { defragmentation }\end{array}$ \\
\hline AK141 & GATCCTTTAACTCTGGCAACCCTC & fwd, $m / s$ for LFH $m n t H$ \\
\hline AK142 & GCCGACTGCGCAAAAGACATAATCG & rev, mls for LFH $m n t H$ \\
\hline AK143 & $\begin{array}{l}\text { GCCGGAACACCATTTCGTCATCAAGGTTTGCATGGGCGCG } \\
\text { GAAGATCAATT }\end{array}$ & $\begin{array}{l}\text { fwd, } y b g E \text {, defragmentation of } \\
y b g E(\mathrm{LFH})\end{array}$ \\
\hline AK144 & $\begin{array}{l}\text { CGATTATGTCTTTTGCGCAGTCGGCTCACACTTCCACTGTCC } \\
\text { AGTTAAACGG }\end{array}$ & $\begin{array}{l}\text { rev, } y b g E \text {, defragmentation of } \\
y b g E(\mathrm{LFH})\end{array}$ \\
\hline AK145 & $\begin{array}{l}\text { GAGGGTTGCCAGAGTTAAAGGATCTTACTCCACAGTAACA } \\
\text { CTCTTCGCAAGGTT }\end{array}$ & $\begin{array}{l}\text { rev, upstream, defragmentation } \\
y b g E(\mathrm{LFH})\end{array}$ \\
\hline AK146 & $\begin{array}{l}\text { CCGTTTAACTGGACAGTGGAAGTGTGATTACTCCACAGTA } \\
\text { АCACTCTTCGCAAGGTT }\end{array}$ & $\begin{array}{l}\text { rev, upstream, defragmentation } \\
y b g E \text { (deletion plasmid) }\end{array}$ \\
\hline AK147 & AAACTCGAGGAGCTGGAGAAAGATTGGGTTCCAAAG & $\begin{array}{l}\text { fwd, ybgE, defragmentation } \\
y b g E \text { (deletion plasmid) }\end{array}$ \\
\hline AK148 & TCACACTTCCACTGTCCAGTTAAACGG & $\begin{array}{l}\text { rev, } y b g E \text {, defragmentation } \\
y b g E \text { (deletion plasmid) }\end{array}$ \\
\hline AK149 & TTTGGATCCCGATACAAATTCCTCGTAGGCGCTC & $\begin{array}{l}\text { rev, kanR for the ligation of } \\
\text { glycolytic cassette fragment }\end{array}$ \\
\hline AK150 & AAAGGATCCAAGTCTACGAGGACCTTACTGATT & $\begin{array}{l}\text { fwd, } n r n A \text { for the ligation of } \\
\text { glycolytic cassette fragment }\end{array}$ \\
\hline AK151 & CCGTCAAACTGACAGTTGCCAAATGG & rev, sequencing yod $F$ \\
\hline AK152 & $\begin{array}{l}\text { GTATAGCATACATTATACGAACGGTAGATCCTAATCGCCA } \\
\text { TCTTCCAGCAG }\end{array}$ & $\begin{array}{l}\text { fwd, } P_{x y / A}-c r e \text { and } x y / R \text { for } \\
\text { integration into } c i t B\end{array}$ \\
\hline AK153 & $\begin{array}{l}\text { CGAAGCACCATTTGAAGGATGCTAACTTATAGGGGTAACA } \\
\text { CTTAAAAAAGAATCAATAACG }\end{array}$ & $\begin{array}{l}\text { rev, } \mathrm{P}_{x y / A}-c r e \text { and } x y / R \text { for } \\
\text { integration into citB }\end{array}$ \\
\hline AK154 & CATCCTTCAAATGGTGCTTCG & fwd, citB down \\
\hline AK155 & CTTGAGAAATCATATAATTGAATCTCATCCCATTG & $\begin{array}{l}\text { fwd, sequencing } x y / R \text { for } \\
\text { integration into } \operatorname{cit} B\end{array}$ \\
\hline AK156 & CGCTGGAGTTTCAATACCGGAGATC & $\begin{array}{l}\text { rev, sequencing cre for } \\
\text { integration into citB }\end{array}$ \\
\hline
\end{tabular}




\begin{tabular}{|c|c|c|}
\hline AK157 & GCTCTCCTGAGAATGTCCGTCTTTC & fwd, Sequencing asns \\
\hline AK158 & CATCGTCACACCTCTTACTGTAAAGGATTG & rev, Sequencing asnS \\
\hline \multirow[t]{2}{*}{ AK159 } & AGCAGCAACTGTTCTTACATACTTTCCCTTGGTATCATTGAT & rev, upstream pGP2270 \\
\hline & GCGGC & \\
\hline AK160 & СТTTCACTTCСАТСАТСТСТGTATCCC & fwd, upstream LFH aapA \\
\hline \multirow[t]{2}{*}{ AK161 } & ССTATCACCTCAAATGGTTCGCTGGGCCTCTAGACAATTTC & rev, upstream LFH aapA \\
\hline & TGTTGCTGG & \\
\hline \multirow[t]{2}{*}{ AK162 } & CGAGCGCCTACGAGGAATTTGTATCGAGGTGCAAACCCGC & fwd, downstream LFH aapA \\
\hline & AGAGGAC & \\
\hline AK163 & АСАTCTCCСCTTATGACAAGACTTTCC & rev, downstream LFH aapA \\
\hline AK164 & CTCAGCCTGATCAAAATGCTCTTCCG & rev, sequencing of LFH aapA \\
\hline AK165 & GGAGGAGCATATGCAGACCGTTC & fwd, sequencing of LFH aapA \\
\hline AK166 & GTCGCAATTGCGTTTGCCGTATCATG & rev, downstream LFH stet \\
\hline \multirow[t]{2}{*}{ AK167 } & CGAGCGCCTACGAGGAATTTGTATCGGCCGGACTGCCTGT & fwd, downstream LFH stet \\
\hline & TTATTACGG & \\
\hline \multirow[t]{2}{*}{ AK168 } & CCTATCACCTCAAATGGTTCGCTGCAAGAGGCCTATCTCTT & rev, upstream LFH stet \\
\hline & TTTTCAAACCG & \\
\hline AK169 & GTTCCTTGCCCTTCGACTGGTTC & fwd, upstream LFH stet \\
\hline AK170 & CTTACCCGAAATGCGACGTGGTG & fwd, sequencing of LFH stet \\
\hline AK171 & GGATCTACGATGTCTTTCGTAATGGTTTTG & rev, sequencing of LFH stet \\
\hline AK172 & GATACACCGTGGTGCCTGGTTTTTG & rev, downstream LFH yfnA \\
\hline \multirow[t]{2}{*}{ AK173 } & CGAGCGCCTACGAGGAATTTGTATCGGGCTTTCATTTGTCA & fwd, downstream LFH yfnA \\
\hline & TCTGGATCGCTG & \\
\hline \multirow[t]{2}{*}{ AK174 } & ССTATCACCTCAAATGGTTCGCTGCTGCGCACTCAATGTTT & rev, upstream LFH yfnA \\
\hline & CAAGCGG & \\
\hline
\end{tabular}




\begin{tabular}{|c|c|c|}
\hline AK176 & GCTGAACACCGCCGCATTGAC & fwd, sequencing of LFH yfnA \\
\hline AK177 & CGTCAGGCTCATGGAGCGTTC & rev, sequencing of LFH yfnA \\
\hline AK178 & CACATGTCAAAACAACTGAAGCAAAAGCTTC & $\begin{array}{l}\text { rev, sequencing of LFH yqiK } \\
\text { (mutated) }\end{array}$ \\
\hline AK179 & GCGCTGGCATTCCCGTAGTTG & $\begin{array}{l}\text { rev, downstream LFH yqiK } \\
\text { (mutated) }\end{array}$ \\
\hline AK180 & $\begin{array}{l}\text { CCTATCACCTCAAATGGTTCGCTGTTGTTAGAAGGAGGCTG } \\
\text { TTTGACGCAG }\end{array}$ & $\begin{array}{l}\text { fwd, downstream LFH yqiK } \\
\text { (mutated) }\end{array}$ \\
\hline AK181 & $\begin{array}{l}\text { CGAGCGCCTACGAGGAATTTGTATCGCTATTCATTTTTCAG } \\
\text { CAATGCCGAAGCC }\end{array}$ & $\begin{array}{l}\text { rev, upstream LFH yqiK } \\
\text { (mutated) }\end{array}$ \\
\hline AK182 & CGATATTCAGTCACAGCTCGTTTCTTC & $\begin{array}{l}\text { fwd, upstream LFH yqiK } \\
\text { (mutated) }\end{array}$ \\
\hline AK183 & CAGCATTCATGTGAATTCAGGGGTTG & $\begin{array}{l}\text { fwd, sequencing of LFH yqiK } \\
\text { (mutated) }\end{array}$ \\
\hline AK184 & GGTTTCACCTCATTCAGAAGATAGACAG & rev, sequencing yqiK \\
\hline
\end{tabular}

\begin{tabular}{|c|c|c|}
\hline AK186 & $\begin{array}{l}\text { CGAGCGCCTACGAGGAATTTGTATCGCCGGTGGGCAAAAA } \\
\text { TCTTTGTCATCG }\end{array}$ & $\begin{array}{l}\text { rev, upstream LFH yqiK } \\
\text { (deletion) }\end{array}$ \\
\hline AK187 & CATCAGGAAGAGGTGGTCATGTGAAAC & $\begin{array}{l}\text { fwd, upstream LFH yqiK } \\
\text { (deletion) }\end{array}$ \\
\hline AK188 & GGAAATGATCGGGTCGTATAGCCC & $\begin{array}{l}\text { fwd, sequencing of LFH yqiK } \\
\text { (deletion) }\end{array}$ \\
\hline AK189 & CATTCCCGTTTTGGAAATTGCTAAACCTG & fwd, sequencing ybec \\
\hline AK190 & GTAACAGGAGTGTTCACCAACTATCC & rev, sequencing ybec \\
\hline AK191 & GACGATTTCAGAGATCCGAAAGTCATATG & fwd, sequencing LFH yveA \\
\hline AK192 & CTTAAGGACTGGCATTACACAAGCGG & fwd, upstream LFH yveA \\
\hline AK193 & $\begin{array}{l}\text { CCTATCACCTCAAATGGTTCGCTGCTTGTTTAGACATTCGCT } \\
\text { TССТССTTTG }\end{array}$ & rev, upstream LFH yveA \\
\hline AK194 & $\begin{array}{l}\text { CGAGCGCCTACGAGGAATTTGTATCGCCCAAAAGCCGCCA } \\
\text { TTGATTATGATAAATAAAG }\end{array}$ & fwd, downstream LFH yveA \\
\hline
\end{tabular}




\begin{tabular}{|c|c|c|}
\hline AK195 & GCTGCTGAGAAGGATTCGCCC & rev, downstream LFH yveA \\
\hline AK196 & CCATGCCATTATTTTTCACCTCCCGG & rev, sequencing LFH yveA \\
\hline AK197 & CAGCCGATGCGGTTGTCCCAG & rev, sequencing LFH ysdA \\
\hline AK198 & GGCTTCTTCCCGGCTTGAAGC & rev, downstream LFH ysdA \\
\hline \multirow[t]{2}{*}{ AK199 } & CGAGCGCCTACGAGGAATTTGTATCGCTCATCGCTATTTAC & fwd, downstream LFH ysdA \\
\hline & TACAGCCCGTTTG & \\
\hline \multirow[t]{2}{*}{ AK200 } & ССTATCACCTCAAATGGTTCGCTGCCGCACAGATTAATCAA & rev, upstream LFH ysdA \\
\hline & CACCAAATAAGC & \\
\hline AK201 & CATGGACTACGGTAAGTTCCGATTTG & fwd, upstream LFH ysdA \\
\hline AK202 & GAATCAAGTCCCGTCAGGAAGCAC & fwd, sequencing LFH ysdA \\
\hline AK203 & GTCCGCACCAGGCCAAATTCC & rev, sequencing of serA \\
\hline AK204 & CAAGCTGTCAGATCATTGATTTATTAGGCTTTAC & fwd, sequencing of serA \\
\hline
\end{tabular}

\begin{tabular}{lll}
\hline AK205 & CTGTTTTCAGCTTCCTGTATTCCATGCCATTTATTACTCCAC & rev, upstream for pGP2270 \\
& AGTAACACTCTTCGC & \\
\hline AK206 & GCATGGAATACAGGAAGCTGAAAACAG & fwd, downstream for pGP2270
\end{tabular}

\begin{tabular}{lll}
\hline AK207 & AAACCATGGGATCATCAAAGTACTCTTCATTCCAAACGG & rev, downstream for pGP2270 \\
\hline AK208 & CCTAATTTATAACCGATGCCCCATAACG & rev, sequencing of pGP2270 \\
& & deletion \\
\hline AK209 & AAAGAATTCGTTGGTTCTTTGTATTCTGGGTGGGG & fwd, promoter $1+2$ of infC for \\
& & pAC5 \\
\hline AK210 & AAAGAATTCGTAAAAGTTGTTCCGGATAAGTCGTCC & fwd, promoter 2 of infC for \\
& & pAC5 \\
\hline AK211 & TTTGGATCCATACCCTCATTAACCAATTGATCTTTGCTAATA & rev, promoter of infC for pAC5, \\
& AT & AUU start codon \\
\hline AK212 & TTTGGATCCATACCCTCATTAACCAATTGATCTTTGCTAATC & rev, promoter of infC for pAC5, \\
& AT & AUG start codon \\
\hline AK213 & CGATATCCAAATTGTAAGATTGACTGGGAC & rev, sequencing infC-rpml-rp/T-
\end{tabular}




\begin{tabular}{|c|c|c|}
\hline AK214 & CTGTATCAGTTTGTTCATGTGTCAGGC & $\begin{array}{l}\text { fwd, sequencing infC-rpml-rp/T- } \\
y s d A \text { operon }\end{array}$ \\
\hline AK215 & $\begin{array}{l}\text { GAGCTTATTAAGTGGTCATTAAATCAAACGTCCAAGACTG } \\
\text { TTTGGCGCGGTACTTTG }\end{array}$ & rev, upstream for pGP2282 \\
\hline AK216 & GACGTTTGATTTAATGACCACTTAATAAGCTC & fwd, downstream for pGP2282 \\
\hline AK217 & TTTCCATGGGACATGGAAGTGATCGGCGTTGC & rev, downstream for pGP2282 \\
\hline AK218 & CCTGTTAACTACATTTGGGGAGGAAG & rev, sequencing of pGP2282 \\
\hline AK219 & $\begin{array}{l}\text { AGCAGCAACTGTTCTTACATACTTTCCCCTTGGTATCATTGA } \\
\text { TGCGGCC }\end{array}$ & rev, upstream for pGP2283 \\
\hline AK220 & TTTCCATGGCGGACAAAACTTGCAAAACAGCCATAC & rev, downstream for pGP2283 \\
\hline AK221 & CTCTTAAATCTGCCCCGTTCTCAAG & fwd, sequencing of $p G P 2284$ \\
\hline AK222 & AAACCATGGCTTCCAAAATCCCTGGCGGCTG & fwd, upstream for pGP2284 \\
\hline AK223 & $\begin{array}{l}\text { TGTATGTCTCTGATTTGGAGGCGCGGATGGTTCGACCGGT } \\
\text { TG }\end{array}$ & rev, upstream for pGP2284 \\
\hline AK224 & CTTCTCCCCCCTCATCCGAAG & fwd, sequencing of tcyK LFH \\
\hline AK225 & GAACCATTCGAATAAAACCGCTACAGC & fwd, downstream tcyK LFH \\
\hline AK226 & $\begin{array}{l}\text { CCGAGCGCCTACGAGGAATTTGTATCGGACGACTATTCCA } \\
\text { AAGAGC }\end{array}$ & rev, downstream tcyK LFH \\
\hline AK227 & $\begin{array}{l}\text { CCTATCACCTCAAATGGTTCGCTGGTTATAAGCGAAAATAA } \\
\text { TATTGCCATGAATGCTG }\end{array}$ & fwd, upstream tcyK LFH \\
\hline AK228 & CCGGGTATGACATTGAAGTGATGAAAG & rev, upstream tcyK LFH \\
\hline AK229 & GCATTACTTGGCGGGGGATGTTC & rev, sequencing of tcyK LFH \\
\hline AK230 & CCTTCGTACCTGTATTTTCATTCCGTATATATG & fwd, sequencing of glyA \\
\hline AK231 & GTGCGGGCGGTTTATGAGTGC & rev, sequencing of glyA \\
\hline AK232 & ATTCATCCGCAAGCCTTGCAGGG & fwd, glyA deletion region \\
\hline
\end{tabular}




\begin{tabular}{|c|c|c|}
\hline AK233 & GTTAGCGGCGAAGTTGACAGAGG & fwd, glyA deletion region \\
\hline AK234 & CTAAATGTAAGCTTGGGATCGTCCATC & fwd, sequencing of glyA \\
\hline AK235 & CATGTTAAACGGCAGGATACCTGCG & fwd, upstream serA LFH \\
\hline \multirow[t]{2}{*}{ AK236 } & CCTATCACCTCAAATGGTTCGCTGGTCTGAGACCAATACTC & rev, upstream serA LFH \\
\hline & GAAACATCG & \\
\hline \multirow[t]{2}{*}{ AK237 } & CGAGCGCCTACGAGGAATTTGTATCGGTGTCTGTGAAGCT & fwd, downstream serA LFH \\
\hline & CATTGATCTGCC & \\
\hline AK238 & GTGCCAGCTGCTCCAAATCCG & rev, downstream serA LFH \\
\hline AK239 & GGAGTCAGAAGTTGATGATTCTATTGCC & rev, sequencing of serA LFH \\
\hline \multirow[t]{2}{*}{ AK240 } & AAAGGATCCCCGATAAAACTTTACTCATCTTTTTATTACTGG & fwd, aapA for $\mathrm{pBQ} 200$ \\
\hline & AG & \\
\hline AK241 & TTTGTCGACTTATTTCACCTTATGTCCTCTGCGGG & rev, aapA for $\mathrm{pBQ} 200$ \\
\hline AK242 & AAAGGATCCCATCATAGGAGGTTAAGGACATGCATAC & fwd, steT for $p B Q 200$ \\
\hline AK243 & TTTGTCGACCGTTTTATCAGCTTGCTTTTCGTTTTTTCATC & rev, steT for $\mathrm{pBQ} 200$ \\
\hline \multirow[t]{2}{*}{ AK244 } & AAAGGATCCCTAGGAGGAACTTTTGATGAGTTCATTATTTA & fwd, $y f n A$ for $p B Q 200$ \\
\hline & G & \\
\hline AK245 & TTTGTCGACACCGCCGGCTGAAAAGAGATTATTTG & rev, yfnA for $\mathrm{pBQ} 200$ \\
\hline AK246 & AAAGGATCCCTAGGGGAGAAGAAGCATGCAAAAAC & fwd, $y \operatorname{tn} A$ for $p B Q 200$ \\
\hline AK247 & TTTGTCGACCTTTTTGTCAGCTGATATTTCGTTCGCTG & rev, ytnA for $\mathrm{pBQ} 200$ \\
\hline AK248 & AAAGGTACCAATGACAAAGATTTTTGGCCCACCGGGG & fwd, yqiK for pGP172 \\
\hline AK249 & TTTGGATCCCCTTCTAACAACTATTCATTTTTCAGCAATGC & rev, yqiK for pGP172 \\
\hline \multirow[t]{2}{*}{ AK250 } & AAAGGTACCAATGAGAAAAAATAGAATACTGGCCTTGTTT & fwd, $g / p Q$ for $p G P 172$ \\
\hline & GTTC & \\
\hline \multirow[t]{2}{*}{ AK251 } & TTTGGATCCGCTTTTAAATAACCCTTTTTTTACTTTGTGGAAAA & rev, glpQ for $p G P 172$ \\
\hline & G & \\
\hline
\end{tabular}




\begin{tabular}{|c|c|c|}
\hline AK252 & AAACCATGGCCTTAAATCTGAAGGGTGAAGATGAACTG & fwd, upstream of pGP2271 \\
\hline AK253 & $\begin{array}{l}\text { CCGGGCGTTTTTCTTATATAACTGCGCGAAAATATGAGGA } \\
\text { GGCTGTTAAACATGTTG }\end{array}$ & rev, upstream of pGP2271 \\
\hline AK254 & CGCAGTTATATAAGAAAAACGCCCGG & fwd, downstream of pGP2271 \\
\hline AK255 & TTTGGATCCGCCGATTCGCAGTCTAGAGAAAATCC & rev, downstream of pGP2271 \\
\hline AK256 & CCGCCGCACTTTGACATTCAACG & rev, sequencing of pGP2271 \\
\hline AK257 & GAATTTGATCCGTCGGCATATCTTCTATTC & fwd, sequencing of pGP2271 \\
\hline AK258 & AAACCATGGCATACGTAGAAGTTGTGATCTCTCCG & fwd, upstream pGP2272 \\
\hline AK259 & $\begin{array}{l}\text { CAAAATGGATGTTTTCACACAGCATGGTTAAGGAGTTCAT } \\
\text { ATGAAACCTTCCTTTATCGTTTTTTG }\end{array}$ & rev, upstream pGP2272 \\
\hline AK260 & CTTAACCATGCTGTGTGAAAACATCCATTTTG & fwd, downstream pGP2272 \\
\hline AK261 & TTTGGATCCCCTGGCGCCTCTTGACGATATG & rev, downstream pGP2272 \\
\hline AK262 & GACTTGAATCGGCTGTATGGGATATTTATG & rev, sequencing pGP2272 \\
\hline AK263 & GATGTTAAAACCGCACAAGTTTCCGC & fwd, sequencing pGP2272 \\
\hline AK264 & AAAGGATCCCAGCGCCCACTTCAGGAAGTTC & $\begin{array}{l}\text { fwd, upstream deletion plasmid } \\
\text { fadE-mrgA }\end{array}$ \\
\hline AK265 & $\begin{array}{l}\text { GTTACCCTAAATAAGAGGAAAGCATCCACGAGGCTGAGA } \\
\text { AATATACTGTCTGATTGG }\end{array}$ & $\begin{array}{l}\text { rev, upstream deletion plasmid } \\
\text { fadE-mrgA }\end{array}$ \\
\hline AK266 & GTGGATGCTTTCCTCTTATTTAGGGTAAC & $\begin{array}{l}\text { fwd, downstream deletion } \\
\text { plasmid fadE-mrgA }\end{array}$ \\
\hline AK267 & TTTCCATGGGTAAAAAAAGTGGCGAGCTTTGGTGAC & $\begin{array}{l}\text { rev, downstream deletion } \\
\text { plasmid fadE-mrgA }\end{array}$ \\
\hline AK268 & GCTGACTGTTACGCTATACAACGGAG & $\begin{array}{l}\text { rev, sequencing deletion } \\
\text { plasmid fadE-mrgA }\end{array}$ \\
\hline AK269 & CAAGATCTTCGTTTACTTCCACAACCG & $\begin{array}{l}\text { fwd, sequencing deletion } \\
\text { plasmid fadE-mrgA }\end{array}$ \\
\hline AK270 & $\begin{array}{l}\text { AATGACGGGCGGAACCGGTTTTCGTTTTTCGCCACTTTCTC } \\
\text { CCTCATAC }\end{array}$ & $\begin{array}{l}\text { rev, upstream deletion plasmid } \\
\text { yflD-yfhF }\end{array}$ \\
\hline
\end{tabular}




\begin{tabular}{|c|c|c|}
\hline AK271 & GAAAACCGGTTCCGCCCGTCATT & $\begin{array}{l}\text { fwd, downstream deletion } \\
\text { plasmid } y f I D-y f h F\end{array}$ \\
\hline AK272 & TTTCCATGGGAGAAGCCCTTGCGCTGAAGC & $\begin{array}{l}\text { rev, downstram deletion } \\
\text { plasmid } y f I D-y f h F\end{array}$ \\
\hline AK273 & CCGTCATAGCGGTATTTTCTAAACGC & $\begin{array}{l}\text { rev, sequencing deletion } \\
\text { plasmid } y f l D-y f h F\end{array}$ \\
\hline AK274 & CGACCAAGAAGCGAGCCCATTC & fwd, upstream ydgF LFH \\
\hline AK275 & $\begin{array}{l}\text { CGAGCGCCTACGAGGAATTTGTATCGCATCAATAAGACTC } \\
\text { AAAACTCСTGССTC }\end{array}$ & rev, upstream ydgF LFH \\
\hline AK276 & $\begin{array}{l}\text { CCTATCACCTCAAATGGTTCGCTGGTCTTTCGTCATGTCGTC } \\
\text { TGTCACTTTATG }\end{array}$ & fwd, downstream ydgF LFH \\
\hline AK277 & GCCTGTTCCCTTGAGATATGCTTGAAG & rev, downstream ydgF LFH \\
\hline AK278 & CCAATCCCTTTTTCGAGCAGCTTTTTC & rev, sequencing ydgF LFH \\
\hline AK279 & GATTTTATCTGGGCATCCGTACGCTTC & $f w d$, sequencing $y d g F$ LFH \\
\hline AK280 & CAATTATGTGAAAGGTGTGCTGATTAGATTG & fwd, sequencing ybgF LFH \\
\hline AK281 & GATTATATGTTTGTGATGGACTACGAAGAGG & $f w d$, upstream ybgF LFH \\
\hline AK282 & $\begin{array}{l}\text { CGAGCGCCTACGAGGAATTTGTATCGCTCATTCAAAATAA } \\
\text { AAAGAACCTGCCTCC }\end{array}$ & rev, upstream ybgF LFH \\
\hline AK283 & $\begin{array}{l}\text { CCTATCACCTCAAATGGTTCGCTGGAGTTCATCTTTTTCCAA } \\
\text { CTTTCTATCAGCG }\end{array}$ & $f w d$, downstream ybgF LFH \\
\hline AK284 & CTATCCAACACATATTAGATACATACCCGC & rev,downstream ybgF LFH \\
\hline AK285 & CATATGGGCTGAACACCTTTCTCTTTTTGC & rev, sequencing ybg $F$ LFH \\
\hline AK286 & GGGCGCAACTGAATTTACTCTGATG & fwd, sequencing yhjB LFH \\
\hline AK287 & GTTGTGATAATTGAAGCTCCCTCCGG & fwd, upstream yhjB LFH \\
\hline AK288 & $\begin{array}{l}\text { CGAGCGCCTACGAGGAATTTGTATCGCCTAAGCATAAAAA } \\
\text { AAGCAATCTGGACACC }\end{array}$ & rev, upstream yhjB LFH \\
\hline AK289 & $\begin{array}{l}\text { CCTATCACCTCAAATGGTTCGCTGCACCGAAGATGATGATG } \\
\text { AGAGCTGC }\end{array}$ & fwd, downstream yhjB LFH \\
\hline
\end{tabular}




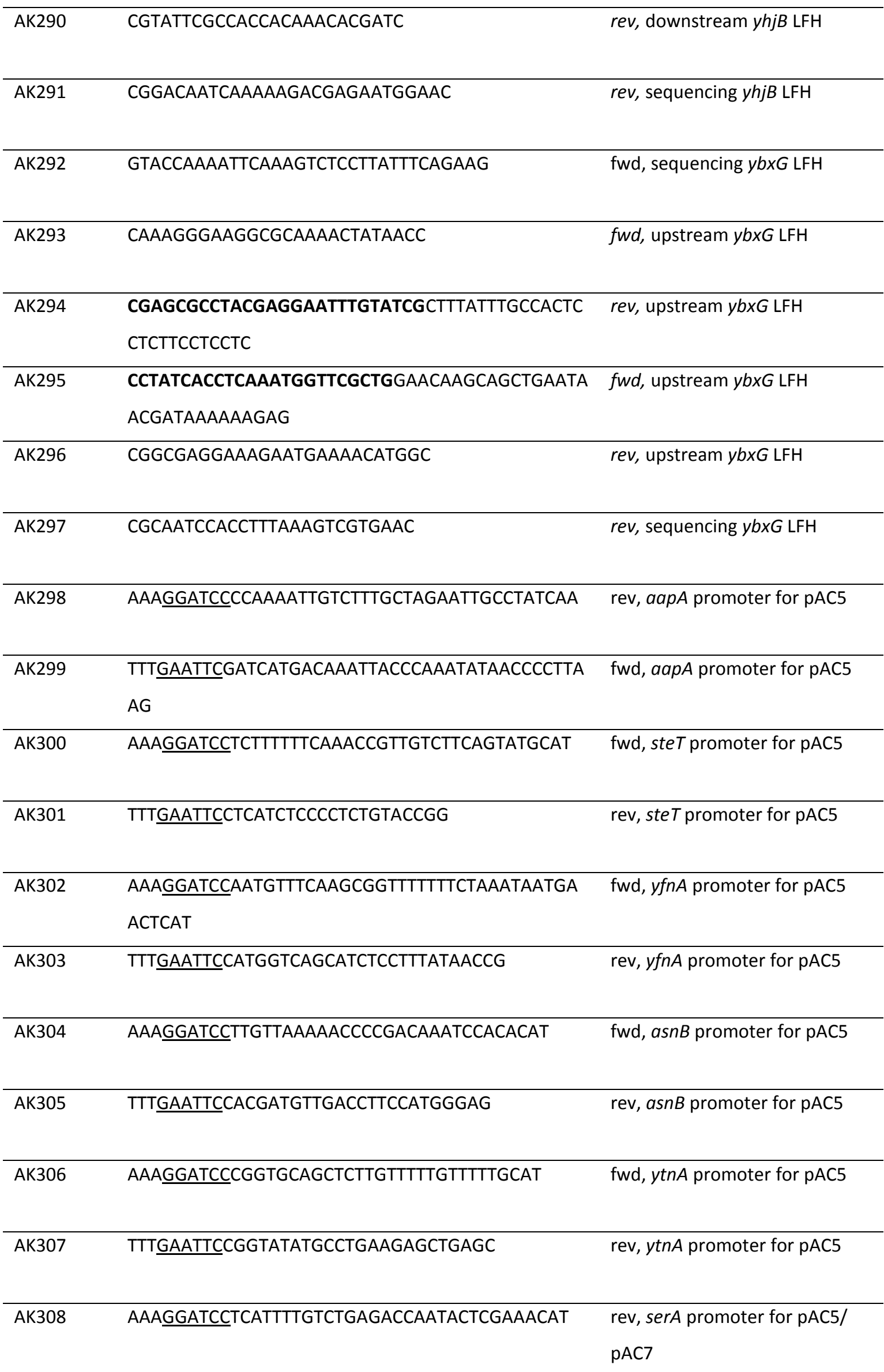




\begin{tabular}{|c|c|c|}
\hline AK309 & TTTGAATTCCCCTGCCCCGAAGATTGATCATATG & $\begin{array}{l}\text { fwd, serA promoter for pAC5/ } \\
\text { pAC7 }\end{array}$ \\
\hline AK310 & AAACTCGAGTATGGCAGAAGTGTCGGGTGCG & $\begin{array}{l}\text { fwd, upstream deletion plasmid } \\
\text { papB-ptsl }\end{array}$ \\
\hline AK311 & $\begin{array}{l}\text { CACCATTAATCCAAGCCCCGTGGCTGCTGGTTTTATTTTAG } \\
\text { GAGTCTTAAAC }\end{array}$ & $\begin{array}{l}\text { rev, upstream deletion plasmid } \\
\text { papB-ptsl }\end{array}$ \\
\hline AK312 & CGAAACGCAATCAAGCCATCCGC & $\begin{array}{l}\text { fwd, sequencing deletion } \\
\text { plasmid papB-ptsl }\end{array}$ \\
\hline AK313 & AAACCATGGGCGTACACGGTTTCGAGCTGATC & fwd, upstream pGP2288 \\
\hline AK314 & GCCCGCCGGATAAAAGAAGCTG & rev, upstream pGP2288 \\
\hline AK315 & $\begin{array}{l}\text { CAGCTTCTTTTATCCGGCGGGCCATCATGTGAGCGTTTTTTT } \\
\text { TTAGTTATCAGAAG }\end{array}$ & fwd, downstream pGP2288 \\
\hline AK316 & TTTGGATCCCAGTCAATTTGGATTAGGAGTAAAAACAGGA & rev, downstream pGP2288 \\
\hline AK317 & GTATTCAAACTGCATTAGAGAAGTCATCAAAC & $\begin{array}{l}\text { fwd, sequencing pGP2288 } \\
\text { insertion }\end{array}$ \\
\hline AK318 & CTAAACCCGTTAACATCAAAACAAACACCC & $\begin{array}{l}\text { rev, sequencing of pGP2288 } \\
\text { insertion }\end{array}$ \\
\hline AK319 & GGTGTCCAGATTGCTTTTTTTAATGCTTAGG & fwd, sequencing yhjCB \\
\hline AK320 & GCCTTTTGAATCATACTGCCCGTTCC & rev, sequencing yhjCB \\
\hline AK321 & AAAGAATTCCCGTTTTTGGAAATTGCTAAACCTGTTGTG & fwd, ybeC promoter for pAC5 \\
\hline AK322 & $\begin{array}{l}\text { TTTGGATCCGAAAACGTTCCCATTCTTCGATGCAATTGATTC } \\
\text { AT }\end{array}$ & rev, ybeC promoter for pAC5 \\
\hline AK323 & AAAGGATCCGCAAGAGAATGTCATCATACATGAAAGGTG & fwd, ybeC for pBQ200 \\
\hline AK324 & TTTGTCGACTTATTCTTTTCCGGCAGCAGCTTCTG & rev, ybeC for $\mathrm{pBQ} 200$ \\
\hline AK325 & $\begin{array}{l}\text { AAAGGATCCAGGGATTTAAAACAAGAAAGGAATCTGTACA } \\
\text { TG }\end{array}$ & rev, ilvA for $\mathrm{pBQ} 200$ \\
\hline AK326 & TTTGTCGACTTAGATTAGCAAATGGAACAAGTCCTCATCC & fwd, ilvA for pBQ200 \\
\hline
\end{tabular}




\begin{tabular}{|c|c|c|}
\hline AK328 & $\begin{array}{l}\text { CCGAGCGCCTACGAGGAATTTGTATCGCAGCGGTTGGAAA } \\
\text { GGAAGCAATATGAG }\end{array}$ & rev, downstream yvoD LFH \\
\hline AK329 & $\begin{array}{l}\text { CCTATCACCTCAAATGGTTCGCTGGACCGGCCAGAAAGAT } \\
\text { СТTСTTCATC }\end{array}$ & fwd, upnstream yvoD LFH \\
\hline AK330 & CGAAATCTTGCAGTCATCATCGAAGTG & rev, upnstream yvoD LFH \\
\hline AK331 & GGCAAGCAATACGACCGGCTC & rev, sequencing yvoD LFH \\
\hline AK332 & CAAATGCCCCGTCAGGCACATC & fwd, sequencing yvoD LFH \\
\hline AK333 & AAAGGATCCGGTGCCAGGCTCTCTATTTTAAAGGG & rev, thrR for $\mathrm{pBQ} 200$ \\
\hline AK334 & $\begin{array}{l}\text { AAAGGATCCTCTTGATTTTTTGAAACATCCTTCATTTTCGCA } \\
\text { C }\end{array}$ & $\begin{array}{l}\text { fwd, promoter spoOB-obg-thrR- } \\
\text { pheA operon }\end{array}$ \\
\hline AK335 & TTTGAATTCGCAATATGCGGAGTAAACACCTAGAATG & $\begin{array}{l}\text { rev, promoter spoOB-obg-thrR- } \\
\text { pheA operon }\end{array}$ \\
\hline AK336 & CTTCAGTTTTTCGCTTCTAAAAAAGGAGTAGG & $\begin{array}{l}\text { rev, sequencing } s p o O B \\
\text { promoter }\end{array}$ \\
\hline AK337 & GTGTTTTTTGTATCTAAAATCCATCAGGGTG & $\begin{array}{l}\text { fwd, sequencing } s p o O B \\
\text { promoter }\end{array}$ \\
\hline AK338 & GGGCGTTATGAACAATTTCTCAATACAGC & rev, sequencing hom operon \\
\hline AK339 & GCTGTGATGTCGTCTGCGGTTATC & fwd, sequencing hom operon \\
\hline AK340 & CTGGAAGACGTCAAAGTAAAGGGG & $\begin{array}{l}\text { rev, sequencing hom operon (in } \\
\text { hom) }\end{array}$ \\
\hline AK341 & CGAAGAGATCAAGCGGCGACAG & rev, sequencing of thrC \\
\hline AK342 & CATTTGGCAGGGATAATAGTGGACAAG & fwd, sequencing of thrC \\
\hline AK343 & CCCGATCAGCTTCATTGTATAGCCG & $\begin{array}{l}\text { fwd, sequencing of hom operon } \\
\text { (in hom) }\end{array}$ \\
\hline AK344 & GAAGTGTTACCTGTTGACGCGCAC & $\begin{array}{l}\text { fwd, sequencing of hom operon } \\
\text { (in } t h r C \text { ) }\end{array}$ \\
\hline AK345 & CGAAAGGCAGCAAGGTCGTAGC & $\begin{array}{l}\text { rev, sequencing hom operon (in } \\
\text { thrC) }\end{array}$ \\
\hline AK346 & TTTGAATTCCGATCACATCTTACTCGAAAACGGAAAG & $\begin{array}{l}\text { fwd, serA promoter for pAC5/ } \\
\text { pAC7 (bigger region) }\end{array}$ \\
\hline
\end{tabular}




\begin{tabular}{|c|c|c|}
\hline AK347 & GCCGGATAATGCGCTGCTGAAAC & fwd, sequencing of $\operatorname{cod} Y$ \\
\hline AK348 & AGAACTACATTTCTCGCCTTGATATAAGCC & rev, sequencing of cod \\
\hline AK349 & TTTCTCGAGTTAGCTCCAACCGTTCCCTTCTACAC & $\begin{array}{l}\text { fwd, hom for } \\
\text { pET-SUMOadapt }\end{array}$ \\
\hline AK350 & $\begin{array}{l}\text { AAAGGTCTCATGGTTTGAAAGCGATTCGTGTAGGGCTTTTA } \\
\text { G }\end{array}$ & $\begin{array}{l}\text { rev, hom for } \\
\text { pET-SUMOadapt }\end{array}$ \\
\hline AK351 & AAAGGATCCTTAGCTCCAACCGTTCCCTTCTACAC & fwd, hom for pGP172 \\
\hline AK352 & TTTGGTACCATTGAAAGCGATTCGTGTAGGGCTTTTAG & rev, hom for pGP172 \\
\hline AK353 & CCCATCCTATAGAAGATAAAGAGGAATGTAC & $\begin{array}{l}\text { rev, sequencing of amplification } \\
\text { boarder yokD-ilvD }\end{array}$ \\
\hline AK354 & CGTTGGAATGGGACTCGGGC & $\begin{array}{l}\text { rev, sequencing of amplification } \\
\text { boarder yokD-ilvD }\end{array}$ \\
\hline AK355 & GATGCGCTGACTGTTACAGGAAAAAC & $\begin{array}{l}\text { rev, sequencing of amplification } \\
\text { boarder yokD-ilvD }\end{array}$ \\
\hline AK356 & CGACAGCCGGCCCTTCGTG & $\begin{array}{l}\text { fwd, sequencing of } \\
\text { amplification boarder ilvD-thyA }\end{array}$ \\
\hline AK357 & GGAAGCGGTCTAACATCTCTTTCCAG & $\begin{array}{l}\text { fwd, sequencing of } \\
\text { amplification boarder ilvD-thyA }\end{array}$ \\
\hline AK358 & $\begin{array}{l}\text { AAAGAATTCTTATTTTTCAGTAAACAGCTTCATTGCTTTCCA } \\
\text { G }\end{array}$ & fwd, ypnP for pGP888 \\
\hline AK359 & TTTGGATCCAATGAAAGCATACGATTTTACACAGGGGAAC & rev, ypnP for pGP888 \\
\hline AK360 & AAAGAATTCTTATGCACGTAATTGTTTTTCATTGGCAGGTA & fwd, ypkP for pGP888 \\
\hline AK361 & TTTGGATCCATTGGTTCGCTACAGCCTTCTAGTGG & rev, ypkP for pGP888 \\
\hline AK362 & $\begin{array}{l}\text { AAAGGATCCTTTGATAGGCAATTCTAGCAAAGACAATTTTG } \\
\text { G }\end{array}$ & fwd, aapA for pGP888 \\
\hline AK363 & TTTGAATTCTTATTTCACCTTATGTCCTCTGCGGG & rev, aapA for pGP888 \\
\hline AK364 & AAAGAATTCTCAGCTTGCTTTTCGTTTTTTCATCCCG & fwd, steT for pGP888 \\
\hline AK365 & $\begin{array}{l}\text { TTTGGATCCAATGCATACTGAAGACAACGGTTTGAAAAAAG } \\
\text { AG }\end{array}$ & rev, steT for $\mathrm{pGP} 888$ \\
\hline
\end{tabular}




\begin{tabular}{|c|c|c|}
\hline AK366 & CCGCTTCATAGAAAGGTTCAGCTTTTAAAATG & fwd, downstream ypnP LFH \\
\hline AK367 & $\begin{array}{l}\text { CGAGCGCCTACGAGGAATTTGTATCGGCAATGAAGCTGTT } \\
\text { TACTGAAAAATAAGTGAAG }\end{array}$ & rev, downstream ypnP LFH \\
\hline AK368 & $\begin{array}{l}\text { CCTATCACCTCAAATGGTTCGCTGCCCCTGTGTAAAATCGT } \\
\text { ATGCTTTCATC }\end{array}$ & fwd, upstream ypnP LFH \\
\hline AK369 & GATTGGAACCATATTGCTGAAAAAGAGCTG & rev, upstream ypnP LFH \\
\hline AK370 & CAATGCTCGCTGATAAATCCCGCTC & fwd. Sequencing ypnP LFH \\
\hline AK371 & CTGAATGATCACGCTGAGCTGATTTATG & rev, sequencing ypnP LFH \\
\hline AK372 & CGGGGGAACGATTCAGCCATATG & fwd, downstream ypkP LFH \\
\hline AK373 & $\begin{array}{l}\text { CGAGCGCCTACGAGGAATTTGTATCGGCTTATATACCTGCC } \\
\text { AATGAAAAACAATTACG }\end{array}$ & rev, downstream ypkP LFH \\
\hline AK374 & $\begin{array}{l}\text { CCTATCACCTCAAATGGTTCGCTGGAAGGCTGTAGCGAAC } \\
\text { CAATTAAAATCC }\end{array}$ & fwd, upstream ypkP LFH \\
\hline AK375 & CTCCCAATGGCGTTCTTGGCG & rev, downstream ypkP LFH \\
\hline AK376 & GTCAGCAATTGTTAATCGATCCCTCC & fwd, sequencing ypkP LFH \\
\hline AK377 & GCTGTGGTTCTTAAAAGGAGATACGAATG & rev, sequencing ypkP LFH \\
\hline AK378 & CTACGACGTCCACATATCTCGGG & fwd, sequencing of $p t s G H I$ LFH \\
\hline AK379 & AAAGAATTCCGTATATAGTTTCAGCAGAATAGAAGGGC & $\begin{array}{l}\text { fwd, promoter of } s d a A B \text { for } \\
\text { pAC5 }\end{array}$ \\
\hline AK380 & TTTGGATCCTTCATGCTATTCCTCCTTATGAACCAGAG & $\begin{array}{l}\text { rev, promoter of } s d a A B \text { for } \\
\text { pAC5 }\end{array}$ \\
\hline AK381 & GAAAGTGTCCCCAGCTATCTAGATTTTC & fwd, sequencing of ilv \\
\hline AK382 & GCAAAAACAGCCCATAAATAAACTGAAAATTGTC & rev, sequencing of ilvD \\
\hline AK383 & GACGAATCTTCCTATATGACAGGGCAG & fwd, sequencing bcaP \\
\hline AK384 & CTATAGATTCATTTGCCAAGAACAGAAAAGAG & rev, sequencing $b c a P$ \\
\hline
\end{tabular}




\begin{tabular}{|c|c|c|}
\hline AK385 & CGGGTGTGAATTATTAGGTAAGCTGTTC & fwd, sequencing $y b x G$ \\
\hline AK386 & GCTTGCTAAAATAGGGAAAATCCATACGC & rev, sequencing $y b x G$ \\
\hline AK387 & AAAGAGCTCTTGAAAGCGATTCGTGTAGGGCTTTTAG & fwd, hom for pGP574 \\
\hline AK388 & TTTGGATCCGCTCCAACCGTTCCCTTCTACAC & rev, hom for pGP574 \\
\hline AK389 & CGAGTGATACGTTTTGCAATAATAGGAACC & fwd, LFH of $p g i$ \\
\hline \multirow[t]{2}{*}{ AK390 } & CCTATCACCTCAAATGGTTCGCTGGGAAGATTAATGTGAG & rev, LFH of $p g i$ \\
\hline & AAAGCTGACTGG & \\
\hline AK391 & CACTATCGTCCCTATAATGAAAAATAAAAACCG & fwd, sequencing of $p g i$ LFH \\
\hline AK392 & AAAGGATCCATGAAAGGGAGCGTTTTTAGGAAGAAAAGC & fwd, bcaP for $\mathrm{pWH} 844$ \\
\hline \multirow[t]{2}{*}{ AK393 } & TTTGTCGACGGTTATTGATTTAATTTTTGAATGCTTTCTTGAA & rev, bcaP for $\mathrm{pWH} 844$ \\
\hline & TACAG & \\
\hline AK394 & AAAGGATCCGTGGCAAATAAAGAATTAAAGAGGGGCC & fwd, $y b x G$ for $p W H 844$ \\
\hline AK395 & TTTGTCGACTTATTCAGCTGCTTGTTCGCTTTTTGTCAAATTG & rev, $y b \times G$ for $\mathrm{pWH} 844$ \\
\hline AK396 & CTGAGGAACGCTTGGCATGATCTATAC & fwd, downstream thrC LFH \\
\hline \multirow[t]{2}{*}{ AK397 } & CGAGCGCCTACGAGGAATTTGTATCGGTAAAAGGAGCGG & rev, downstream thrC LFH \\
\hline & CCCGTGTATG & \\
\hline \multirow[t]{2}{*}{ AK398 } & CCTATCACCTCAAATGGTTCGCTGGATAAGTCCTTTCCACA & fwd, upstream thrC LFH \\
\hline & TTAGCTCCAAC & \\
\hline AK399 & GCCGAAGCGAAGGAAAATGGATGC & rev, upstream thrC LFH \\
\hline AK400 & CATTGATGATCCAGATGTTGATGTCGTC & rev, sequencing thrC LFH \\
\hline AK401 & CATATGATGGAAAAGTTCTACAAGGAGTG & fwd, downstream yvbW LFH \\
\hline \multirow[t]{2}{*}{ AK402 } & CGAGCGCCTACGAGGAATTTGTATCGCGCAAGCATCAGTA & rev, downstream yvbW LFH \\
\hline & AATAAGAAACCCTC & \\
\hline \multirow[t]{2}{*}{ AK403 } & CCTATCACCTCAAATGGTTCGCTGGTCGTTTTTCATCCTCTT & fwd, upstream yvbW LFH \\
\hline & СТАССТСТСС & \\
\hline
\end{tabular}




\begin{tabular}{|c|c|c|}
\hline AK404 & GATTTTGAAGCAGTAAATCCAGAAGACCG & rev, upstream yvbW LFH \\
\hline AK405 & CAGCGAAAAAACGATTCACAGATCAGTTAATTAC & rev, sequencing yvbW LFH \\
\hline AK406 & CAGCTATTTTCACAAAAACCTTTAATTGAGTAATG & fwd, sequencing yvbW LFH \\
\hline AK407 & GCTTACTGAAAACGGCTCGAAGGTC & fwd, upstream yecA LFH \\
\hline \multirow[t]{2}{*}{ AK408 } & CGAGCGCCTACGAGGAATTTGTATCGCATGATGCCCCCTCT & rev, upstream yecA LFH \\
\hline & CTGATTGATG & \\
\hline \multirow[t]{2}{*}{ AK409 } & ССTATCACCTCAAATGGTTCGCTGCGTTTTGTGATCAAGCT & fwd, downstream yecA LF \\
\hline & TTTCCATTTATCCG & \\
\hline AK410 & CGTTTTCTCTGTTTCTTTGTAGCTTGCATAC & rev, downstream yecA LFH \\
\hline AK411 & CATCACCTGTCATCGCTTCATGATCAC & fwd, sequencing yecA LFH \\
\hline AK412 & CGGTCCGCCATGCTGTAGAAACG & rev, sequencing yecA LFH \\
\hline AK413 & AAATCTAGAAGTGACAGACGACATGACGAAAGAC & fwd, $y d g F$ for $p G P 888$ \\
\hline \multirow[t]{2}{*}{ AK414 } & TTTGGTACCCTTATTGATGCTTCGCTTTTCTCACTTTATAAAT & rev, $y d g F$ for pGP888 \\
\hline & $\mathrm{C}$ & \\
\hline AK415 & AAATCTAGAAATGCAAGGGAATCTGACTGCACTTC & fwd, yodF for pGP888 \\
\hline AK416 & TTTGGTACCGTTTTTTTATGCGCCCTTTTGATTTGGATTAG & rev, yodF for pGP8\& \\
\hline
\end{tabular}

\subsubsection{Oligonucleotides used in this work}

\begin{tabular}{lll}
\hline Name & Sequence & Purpose \\
\hline $\begin{array}{l}\text { cat check } \\
\text { fwd }\end{array}$ & CTAATGTCACTAACCTGCCC & $\begin{array}{l}\text { Sequencing out of cat resistance } \\
\text { cassette }\end{array}$ \\
\hline cat check & GTCTGCTTTCTTCATTAGAATCAATCC & Sequencing out of cat resistance \\
rev & & cassette \\
\hline cat fwd & CGGCAATAGTTACCCTTATTATCAAG & Amplification of cat resistance cassette \\
(kan) & & \\
\hline cat rev & CCAGCGTGGACCGGCGAGGCTAGTTACCC & Amplification of cat resistance cassette \\
(kan) & &
\end{tabular}




\begin{tabular}{llc}
\hline CZ114 & CAGAATTAAAACAAGCATGGC & rev, downstream citZ-icd-mdh deletion \\
\hline CZ116 & CATGTCCTAGCTTATCAGAAC & fwd, upstream citZ-icd-mdh deletion \\
\hline CZ119 & GAAAACAATATGCAACTTTAAATC & rev, downstream sucCD deletion \\
\hline CZ120 & GATACAACAGCAGTTGCTTTG & rev, downstream sdhCAB deletion \\
\hline CZ123 & CTCATTTTCTCTTCATTTCATGC & fwd, upstream sdhCAB deletion
\end{tabular}

\begin{tabular}{lll}
\hline CZ126 & CAGCGAACCATTTGAGGTGATAGGGAACGATGACC & $\begin{array}{l}\text { Sequencing out of phleo resistance } \\
\text { cassette }\end{array}$ \\
\hline TCTAATAATTG & CGATACAAATTCCTCGTAGGCGCTCGGGTAGTATTT & Sequencing out of phleo resistance \\
& TTTGAGAAGATCAC & cassette \\
\hline CZ128 & CCAAAGTGAAACCTAGTTTATC & Sequencing phleo resistance
\end{tabular}

\begin{tabular}{lll}
\hline CZ129 & CGAGACTTTGCAGTAATTGATC & Sequencing phleo resistance \\
& & \\
\hline CZ135 & CAACAGTCGTAAGCGAAAATG & Check PCR sdhCAB deletion
\end{tabular}

\begin{tabular}{lll}
\hline CZ140 & GTATACGAAGAGAGATTAGAAG & rev, downstream citB deletion \\
\hline CZ143 & GCTGTTTATCTTCTCCTGAAG & fwd, upstream citB deletion
\end{tabular}

\begin{tabular}{|c|c|c|}
\hline CZ145 & GGGTATGGCTGACGGCAAAG & Check PCR odhAB deletion \\
\hline CZ151 & CAAGAACATTTTAATTAAGTTTACATC & Check PCR odhAB deletion \\
\hline CZ154 & GTTTGTCTGTCCATTGGGTTC & Check PCR citRA deletion \\
\hline CZ171 & GAAAATACGACACAACGATCAG & Check PCR citRA deletion \\
\hline \multirow[t]{2}{*}{ CZ200 } & TACCGTTCGTATAGCATACATTAATACGAAGTTATCC & Amplification of kan-lox from pGP2514 \\
\hline & TTCCCAGCGAACCATTTGAGGTGATAGGTAAG & \\
\hline \multirow[t]{2}{*}{ CZ201 } & TACCGTTCGTATAATGTATGCTATCGAAGTTATGTA & Amplification of kan-lox from pGP2514 \\
\hline & ACGATCGATACAAATTCCTCGTAGGCGCTCGGGAC & \\
\hline \multirow[t]{2}{*}{ CZ202 } & CTACATAAGAGGACATTCGAC & amplification of the glycolytic cassette \\
\hline & & upstream fragement \\
\hline
\end{tabular}




\begin{tabular}{|c|c|c|}
\hline CZ203 & $\begin{array}{l}\text { GCGCGCCTTCACTTGACAACATCGATATTGGCTGTA } \\
\text { TCAAC }\end{array}$ & $\begin{array}{l}\text { amplification of the glycolytic cassette } \\
\text { upstream fragement }\end{array}$ \\
\hline CZ204 & $\begin{array}{l}\text { TTGTCAAGTGAAGGCGCGCTATCGTACAATACAGCT } \\
\text { TGGAAATAGAGGAGGTCAATTCTTATGTTTAAAGC }\end{array}$ & Amplification of $p t s G H I$ \\
\hline CZ205 & $\begin{array}{l}\text { CCTATCACCTCAAATGGTTCGCTGGTTAAATTGGTTT } \\
\text { GACATACTATC }\end{array}$ & Amplification of $p t s G H I$ \\
\hline CZ236 & GTTTTAGTTCCAGCAGCCAG & Check PCR citZ-icd-mdh deletion \\
\hline DR343 & TTTGGATCCCAAGCGCCGCATATGACTG & check of deletion with pGP2073 \\
\hline DR348 & CCTGTCCTTGGATAGCGTAC & check of deletion with pGP2073 \\
\hline DR377 & TTTGGATCCCTGACAATCACAAACGTACCTGG & construction of $\mathrm{pGP} 2270$ \\
\hline DR381 & CGTGCCGGTTGAAGTGCAT & check of deletion with pGP2270 \\
\hline DR387 & TTTGGATCCGTTCGAATCCTGCCTGTGGA & construction of pGP2283 \\
\hline DR391 & GAATATGGCAAGCCTATGTTACATTAT & check of deletion with pGP2283 \\
\hline DR393 & TTTGGATCCCGGACTCTCTATTATCCTCGTAA & construction of pGP2282 \\
\hline DR397 & GGGTATCTTTTTGATCGTATTATGCT & check of deletion with pGP2282 \\
\hline DR402 & TTTGGATCCCGCAGTCGGAAAAAGCACGA & construction of pGP2284 \\
\hline DR491 & CCATTACGTTTTTTCCACCAGTCTT & check of deletion with pGP2088 \\
\hline DR492 & GGGCGCTCAAATCTTCCACA & check of deletion with pGP2088 \\
\hline DR529 & CGTACGAAATCAGAGCCGCAA & check of deletion with pGP2093 \\
\hline DR530 & GTAAATAGACTGCCAGCGTCCT & check of deletion with pGP2093 \\
\hline DR535 & CCCGAAAATCCGCGCGC & check of deletion with pGP2094 \\
\hline
\end{tabular}




\begin{tabular}{|c|c|c|}
\hline DR591 & TGTACGAGACCTCCTTCCATG & check of deletion with pGP2098 \\
\hline DR592 & AAAAGGAGACTTTTTCTCAGCTGATC & check of deletion with pGP2098 \\
\hline DR607 & TGCGGAAGTAAGCTCTTTCTCTG & check of deletion with pJOE9256 \\
\hline DR608 & TGGCTGCTGTGATGAACTTTGTC & check of deletion with pJOE9256 \\
\hline DR615 & $\begin{array}{l}\text { CGAGCGCCTACGAGGAATTTGTATCGAAGTCTACG } \\
\text { AGGACCTTACTGATT }\end{array}$ & Amplification of $n r n A$ \\
\hline DR616 & ATAGACATGTGCGTCCTGATCC & Amplification of $n r n A$ \\
\hline FC121 & $\begin{array}{l}\text { CATTTATAGTAAAAAGAGAAAGGCTGTATTAAGCAA } \\
\text { GCC }\end{array}$ & Check PCR citZ-icd-mdh deletion \\
\hline FC50 & GTCATATCCTAGCAGGCCTCCGG & Check PCR citG deletion \\
\hline FC53 & CGACCAAAATTGCCACACGGCCG & Check PCR citG deletion \\
\hline FC60 & GAACGTTTCTCTCAGGAAGTTCCTCG & Check PCR sucCD deletion \\
\hline FM86 & CTGCCTTAAGAGCATCGCATGAGGTA & Check PCR sucCD deletion \\
\hline FM111 & CAACAGATAGGTTTCTCAAAAGGAGGGG & Check PCR citB deletion \\
\hline FM165 & $\begin{array}{l}\text { AAAGAGCTCTGATCTGAAGGGGGATTTTGGAGAAT } \\
\text { GG }\end{array}$ & Check PCR citB deletion \\
\hline FM172 & TTGTCCATCCCTCACTCAAGGATCTC & Check PCR sdhCAB deletion \\
\hline JR154 & GCAAAATCAACTTCGCCTGCA & Sequencing of thrR \\
\hline JR155 & CGATGGAAAATGAAGAAGTGCCAT & Sequencing of thrR \\
\hline $\begin{array}{l}\text { kan check } \\
\text { fwd }\end{array}$ & CATCCGCAACTGTCCATACTCTG & $\begin{array}{l}\text { Sequencing out of kan resistance } \\
\text { cassette }\end{array}$ \\
\hline $\begin{array}{l}\text { kan check } \\
\text { rev }\end{array}$ & СТGССТССТСАТССТСТTСАТСС & $\begin{array}{l}\text { Sequencing out of kan resistance } \\
\text { cassette }\end{array}$ \\
\hline kan-fwd & CAGCGAACCATTTGAGGTGATAGG & $\begin{array}{l}\text { Amplification of kan resistance } \\
\text { cassette }\end{array}$ \\
\hline
\end{tabular}




\begin{tabular}{|c|c|c|}
\hline kan-rev & CGATACAAATTCCTCGTAGGCGCTCGG & $\begin{array}{l}\text { Amplification of kan resistance } \\
\text { cassette }\end{array}$ \\
\hline $\begin{array}{l}\text { M13_puc_ } \\
\text { for }\end{array}$ & GTAAAACGACGGCCAGTG & Sequencing of $\mathrm{pBQ} 200$ \\
\hline $\begin{array}{l}\text { M13_puc_ } \\
\text { rev }\end{array}$ & GGAAACAGCTATGACCATG & Sequencing of $\mathrm{pBQ} 200$ \\
\hline MD113 & $\begin{array}{l}\text { CCGAGCGCCTACGAGGAATTTGTATCGTTCTGTTTC } \\
\text { CGGCCCAATACC }\end{array}$ & sequencing sdaCAB suppressors \\
\hline ML84 & CTAATGGGTGCTTTAGTTGAAGA & Cat check up-fragment \\
\hline ML85 & CTCTATTCAGGAATTGTCAGATAG & Cat check down-fragment \\
\hline ML107 & GCTTCATAGAGTAATTCTGTAAAGG & sequencing pAC7 plasmid \\
\hline ML244 & $\begin{array}{l}\text { CTAATACGACTCACTATAGGGAGAGGATATGTGCAC } \\
\text { TTCGCTGCTGCTCAAT }\end{array}$ & sequencing serA deletion \\
\hline MT24 & AAAAGAATTCATGAATATGCGGGCGCAGAAGCT & amplification of hom promoter \\
\hline MT25 & $\begin{array}{l}\text { AAAAGGATCCTTCAAAAAAACTCCACCTTTCTTTTGA } \\
\text { TTGTCC }\end{array}$ & amplification of hom promoter \\
\hline pAC5F & GCGTAGCGAAAAATCCTTTTC & sequencing pAC5 plasmid \\
\hline SH71 & AACGGTGGTATATCCAGTG & sequencing $\mathrm{pWH} 844$ \\
\hline $\begin{array}{l}\text { spec check } \\
\text { fwd }\end{array}$ & GTTATCTTGGAGAGAATATTGAATGGAC & $\begin{array}{l}\text { Sequencing out of spec resistance } \\
\text { cassette }\end{array}$ \\
\hline $\begin{array}{l}\text { spec check } \\
\text { rev }\end{array}$ & CGTATGTATTCAAATATATCCTCCTCAC & $\begin{array}{l}\text { Sequencing out of spec resistance } \\
\text { cassette }\end{array}$ \\
\hline $\begin{array}{l}\text { spec-fwd } \\
\text { (kan) }\end{array}$ & $\begin{array}{l}\text { CAGCGAACCATTTGAGGTGATAGGGACTGGCTCGC } \\
\text { TAATAACGTAACGTGACTGGCAAGAG }\end{array}$ & $\begin{array}{l}\text { Amplification of spec resistance } \\
\text { cassette }\end{array}$ \\
\hline $\begin{array}{l}\text { spec-rev } \\
\text { (kan) }\end{array}$ & $\begin{array}{l}\text { CGATACAAATTCCTCGTAGGCGCTCGGCGTAGCGA } \\
\text { GGGCAAGGGTTTATTGTTTTCTAAAATCTG }\end{array}$ & $\begin{array}{l}\text { Amplification of spec resistance } \\
\text { cassette }\end{array}$ \\
\hline T7-Prom. & TAATACGACTCACTATAGGG & $\begin{array}{l}\text { Sequencing of pGP574 and pET- } \\
\text { SUMOadapt }\end{array}$ \\
\hline T7-Term. & GCTAGTTATTGCTCAGCGG & $\begin{array}{l}\text { Sequencing of pGP574 and pET- } \\
\text { SUMOadapt }\end{array}$ \\
\hline Tc fwd2 & GCTTATCAACGTAGTAAGCGTGG & $\begin{array}{l}\text { Sequencing out of tet resistance } \\
\text { cassette }\end{array}$ \\
\hline
\end{tabular}




\begin{tabular}{lll}
\hline Tc rev & GAACTCTCTCCCAAAGTTGATCCC & $\begin{array}{l}\text { Sequencing out of tet resistance } \\
\text { cassette }\end{array}$ \\
\hline $\begin{array}{l}\text { Tc check } \\
\mathrm{fwd}\end{array}$ & CGGCTACATTGGTGGGATACTTGTTG & Amplification of tet resistance cassette \\
\hline
\end{tabular}




\subsection{Supplementary information}

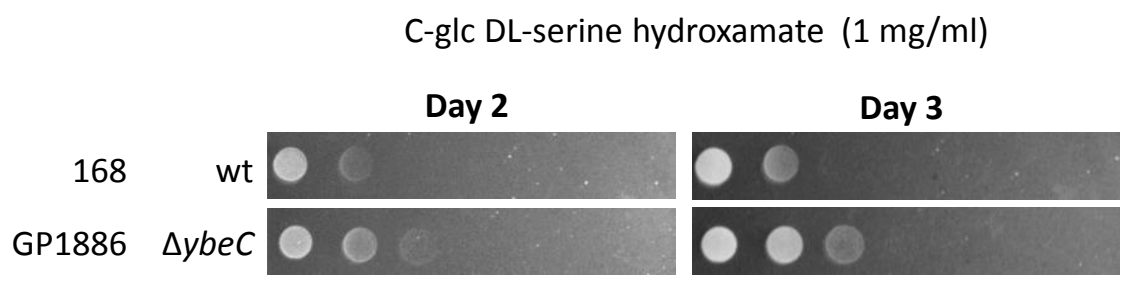

Figure 54. The growth of the wild type strain 168 and the $\Delta y b e C$ strain on C-glc with DL-serine hydroxamate $(1 \mathrm{mg} / \mathrm{ml})$. A serial dilution of the wild type strain and the strain GP1886 was performed in C-glc liquid medium and the cells were plated on C-glc plates with $1 \mathrm{mg} / \mathrm{ml} \mathrm{DL}$-serine hydroxamate.

Table 18: The complete list of genes, included in the MiniBacillus blueprint 2.0

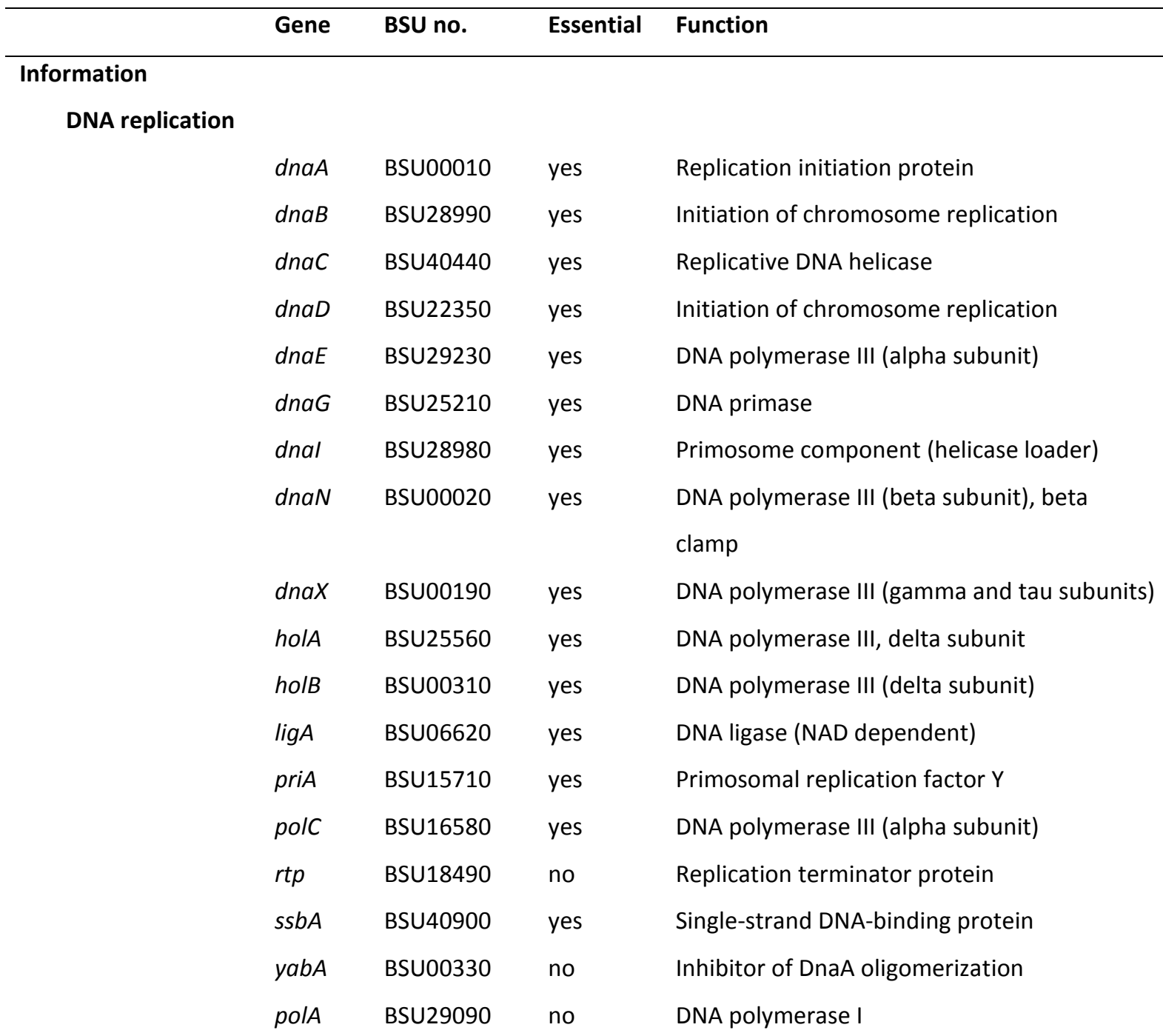

\section{Chromosome maintenance}

scpA BSU23220 yes DNA segregation/condensation protein 


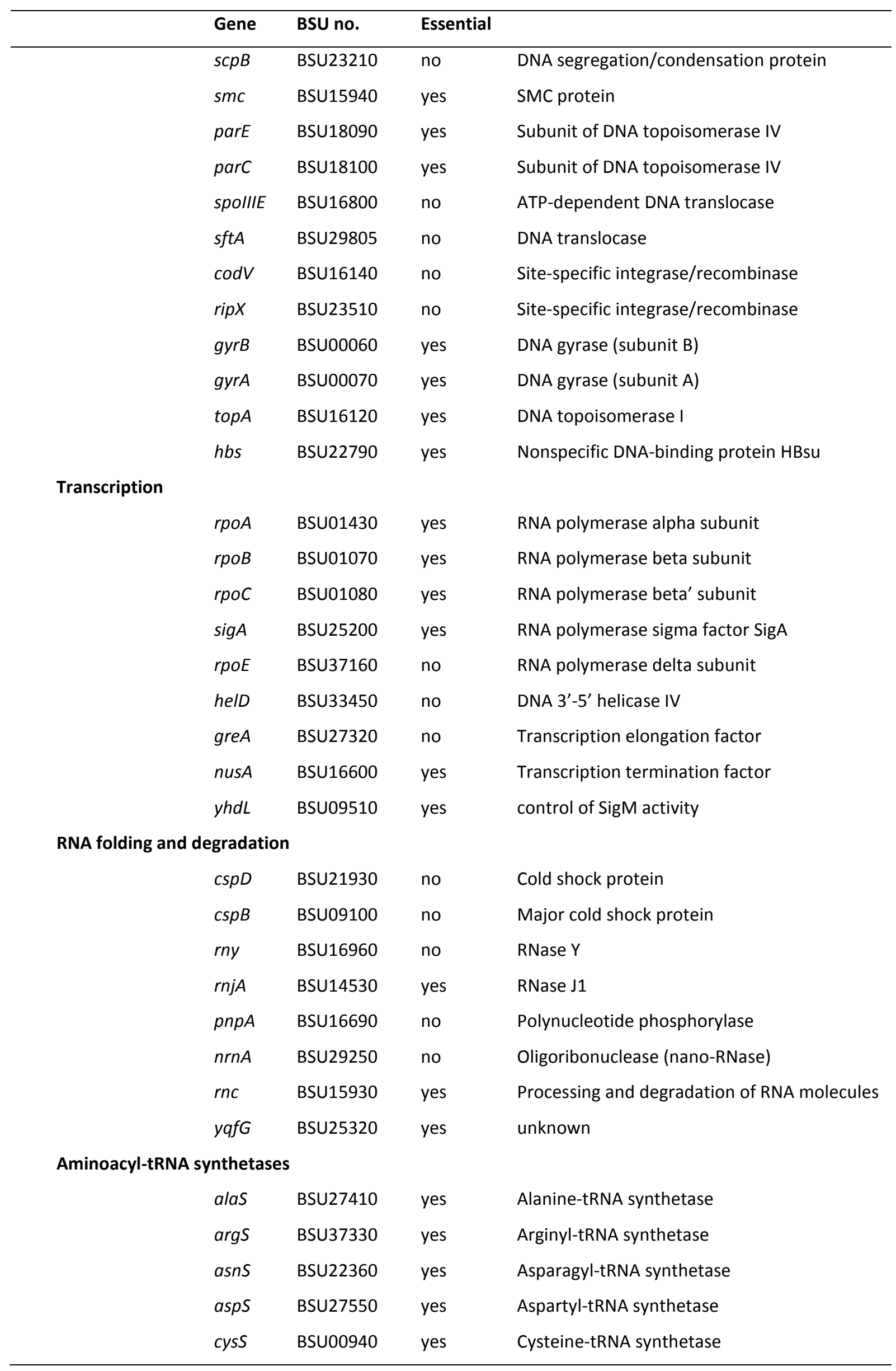




\begin{tabular}{|c|c|c|c|}
\hline Gene & BSU no. & Essential & Function \\
\hline gatC & BSU06670 & yes & Production of glutamyl-tRNA ${ }^{\text {Gln }}$ \\
\hline gatA & BSU06680 & yes & Production of glutamyl-tRNA ${ }^{\text {Gln }}$ \\
\hline gatB & BSU06690 & yes & Production of glutamyl-tRNA ${ }^{\text {Gln }}$ \\
\hline gltX & BSU00920 & yes & Glutamyl-tRNA synthetase \\
\hline glys & BSU25260 & yes & Glycyl-tRNA synthetase (beta subunit) \\
\hline glyQ & BSU25270 & yes & Glycyl-tRNA synthetase (alpha subunit) \\
\hline hisS & BSU27560 & yes & Histidyl-tRNA synthetase \\
\hline iles & BSU15430 & yes & Isoleucyl-tRNA synthetase \\
\hline leus & BSU30320 & yes & Leucyl-tRNA synthetase \\
\hline lysS & BSU00820 & yes & Lysyl-tRNA synthetase \\
\hline mets & BSU00380 & yes & Methionyl-tRNA synthetase \\
\hline phet & BSU28630 & yes & Phenylalanyl-tRNA synthetase (beta subunit) \\
\hline phes & BSU28640 & yes & Phenylalanyl-tRNA synthetase (alpha subunit) \\
\hline pros & BSU16570 & yes & Prolyl-tRNA synthetase \\
\hline sers & BSU00130 & yes & Seryl-tRNA synthetase \\
\hline thrs & BSU28950 & no & Threonyl-tRNA synthetase (major) \\
\hline $\operatorname{trpS}$ & BSU11420 & yes & Tryptophanyl-tRNA synthetase \\
\hline tyrs & BSU29670 & yes & Tyrosyl-tRNA synthetase (major) \\
\hline vals & BSU28090 & yes & Valyl-tRNA synthetase \\
\hline \multicolumn{4}{|l|}{ Ribosomal proteins } \\
\hline$r p / A$ & BSU01030 & no & Ribosomal protein L1 \\
\hline$r p / B$ & BSU01190 & yes & Ribosomal protein L2 \\
\hline rp/C & BSU01160 & yes & Ribosomal protein L3 \\
\hline$r p / D$ & BSU01170 & yes & Ribosomal protein L4 \\
\hline$r p / E$ & BSU01280 & yes & Ribosomal protein L5 \\
\hline$r p / F$ & BSU01310 & yes & Ribosomal protein L6 \\
\hline rpll & BSU40500 & no & Ribosomal protein L9 \\
\hline rplJ & BSU01040 & yes & Ribosomal protein L10 \\
\hline$r p / K$ & BSU01020 & no & Ribosomal protein L11 \\
\hline$r p / L$ & BSU01050 & yes & Ribosomal protein L12 \\
\hline$r p / M$ & BSU01490 & yes & Ribosomal protein L13 \\
\hline$r p / N$ & BSU01260 & yes & Ribosomal protein L14 \\
\hline rplo & BSU01350 & no & Ribosomal protein L15 \\
\hline$r p / P$ & BSU01230 & yes & Ribosomal protein L16 \\
\hline$r p / Q$ & BSU01440 & yes & Ribosomal protein L17 \\
\hline$r p / R$ & BSU01320 & yes & Ribosomal protein L18 \\
\hline rp/s & BSU16040 & yes & Ribosomal protein L19 \\
\hline
\end{tabular}




\begin{tabular}{|c|c|c|c|}
\hline Gene & BSU no. & Essential & Function \\
\hline$r p / T$ & BSU28850 & yes & Ribosomal protein L20 \\
\hline rp/U & BSU27960 & yes & Ribosomal protein L21 \\
\hline$r p / V$ & BSU01210 & yes & Ribosomal protein L22 \\
\hline$r p / W$ & BSU01180 & yes & Ribosomal protein L23 \\
\hline$r p / X$ & BSU01270 & yes & Ribosomal protein L24 \\
\hline rpmA & BSU2794 & yes & Ribosomal protein L27 \\
\hline rpmB & BSU15820 & no & Ribosomal protein L28 \\
\hline rpmC & BSU01240 & no & Ribosomal protein L29 \\
\hline$r p m D$ & BSU01340 & yes & Ribosomal protein L30 \\
\hline rpmE & BSU37070 & no & Ribosomal protein L31 \\
\hline rpmF & BSU15080 & no & Ribosomal protein L32 \\
\hline rpmGA & BSU24900 & no & Ribosomal protein L33a \\
\hline$r p m G B$ & BSU00990 & no & Ribosomal protein L33b \\
\hline rpmH & BSU41060 & no & Ribosomal protein L34 \\
\hline rpml & BSU28860 & no & Ribosomal protein L35 \\
\hline rpmJ & BSU01400 & no & Ribosomal protein L36 \\
\hline$r p s B$ & BSU16490 & yes & Ribosomal protein S2 \\
\hline $\operatorname{rps} C$ & BSU01220 & yes & Ribosomal protein S3 \\
\hline$r p s D$ & BSU29660 & yes & Ribosomal protein S4 \\
\hline rpsE & BSU01330 & yes & Ribosomal protein S5 \\
\hline$r p s F$ & BSU40910 & no & Ribosomal protein S6 \\
\hline rpsG & BSU01110 & yes & Ribosomal protein S7 \\
\hline rpsH & BSU01300 & yes & Ribosomal protein S8 \\
\hline rpsl & BSU01500 & yes & Ribosomal protein S9 \\
\hline rpsJ & BSU01150 & yes & Ribosomal protein S10 \\
\hline rpsK & BSU01420 & yes & Ribosomal protein S11 \\
\hline$r p s L$ & BSU01100 & yes & Ribosomal protein S12 \\
\hline rpsM & BSU01410 & yes & Ribosomal protein S13 \\
\hline $\operatorname{rps} N$ & BSU01290 & yes & Ribosomal protein S14 \\
\hline rpsO & BSU16680 & yes & Ribosomal protein S15 \\
\hline rpsP & BSU15990 & yes & Ribosomal protein S16 \\
\hline$r p s Q$ & BSU01250 & yes & Ribosomal protein S17 \\
\hline rpsR & BSU40890 & yes & Ribosomal protein S18 \\
\hline rpsS & BSU01200 & yes & Ribosomal protein S19 \\
\hline rpsT & BSU25550 & no & Ribosomal protein S20 \\
\hline$r p s U$ & BSU25410 & no & Ribosomal protein S21 \\
\hline
\end{tabular}




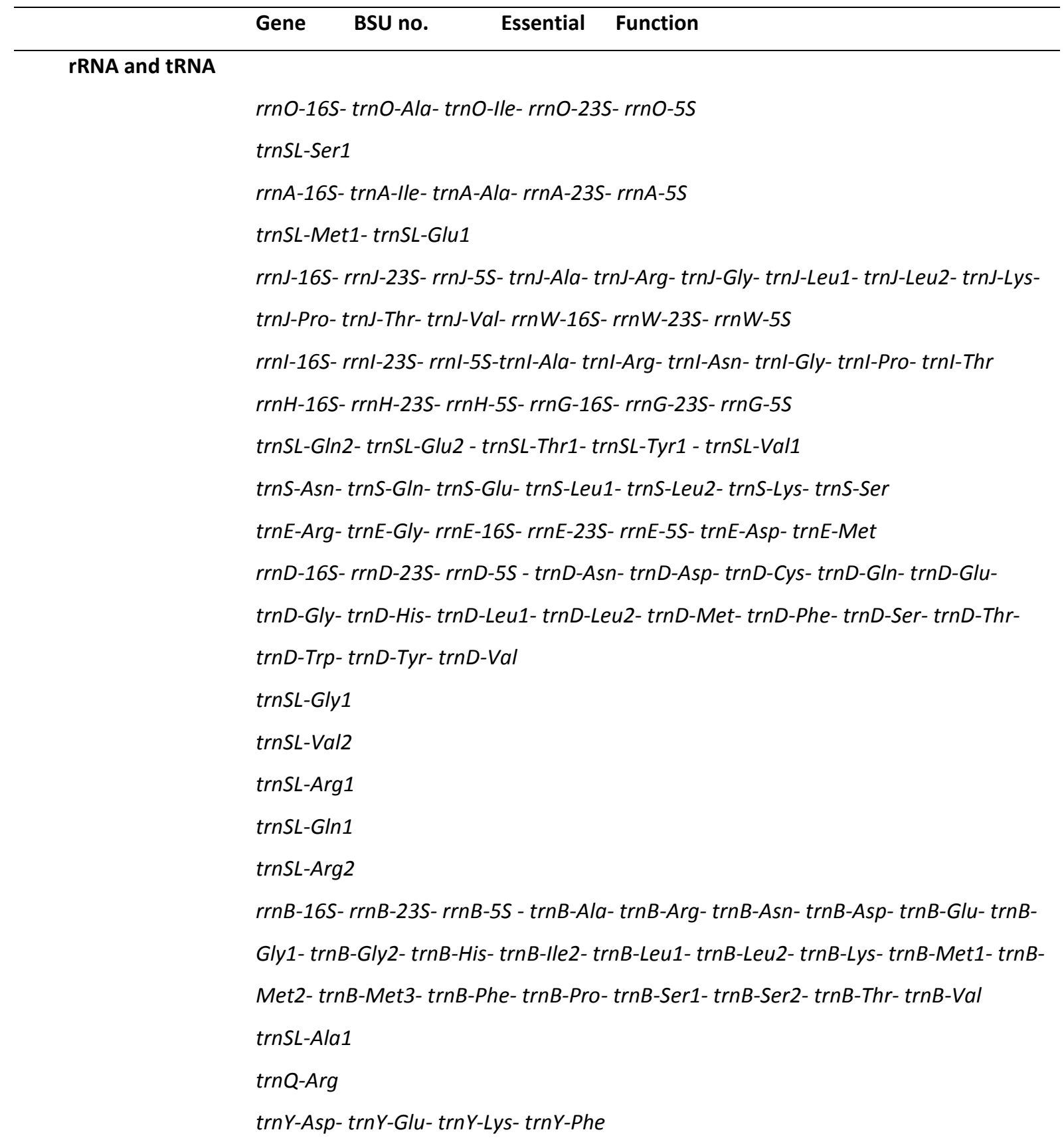

rRNA/ tRNA maturation and modification

$\begin{array}{llll}r n p A & \text { BSU41050 } & \text { yes } & \text { Protein component of RNase P } \\ r n p B & \text { BSU_misc_R } & \text { yes } & \text { RNA component of RNase P } \\ & \text { NA_35 } & & \\ r n z & \text { BSU23840 } & \text { yes } & \text { RNase Z } \\ r p h & \text { BSU28370 } & \text { no } & \text { RNase PH } \\ r b f A & \text { BSU16650 } & \text { no } & \text { Ribosome-binding factor A } \\ r i m M & \text { BSU16020 } & \text { no } & \text { 16S rRNA-processing protein, RNase } \\ c c a & \text { BSU22450 } & \text { yes } & \text { tRNA nucleotidyltransferase } \\ f m t & \text { BSU15730 } & \text { yes } & \text { Methionyl-tRNA formyltransferase }\end{array}$




\begin{tabular}{|c|c|c|c|}
\hline Gene & BSU no. & Essential & Function \\
\hline folD & BSU24310 & yes & Methylenetetrahydrofolate dehydrogenase \\
\hline $\operatorname{rlm} C D$ & BSU06730 & no & rRNA methyltransferase \\
\hline$y s g A$ & BSU28650 & yes & Similar to rRNA methylase \\
\hline mraW & BSU15140 & no & SAM-dependent methyltransferase \\
\hline $\operatorname{csp} R$ & BSU08930 & yes & Similar to tRNA (Um34/Cm34) \\
\hline & & & methyltransferase \\
\hline $\operatorname{trmD}$ & BSU16030 & yes & tRNA methyltransferase \\
\hline $\operatorname{trm} U$ & BSU27500 & yes & tRNA(5-methylaminomethyl-2- thiouridylate) \\
\hline & & & methyltransferase \\
\hline yrvo & BSU27510 & yes & Cysteine desulfurase \\
\hline yaco & BSU00960 & no & Putative 23S rRNA methyltransferase \\
\hline$k s g A$ & BSU00420 & no & rRNA adenine dimethyltransferase \\
\hline rluB & BSU23160 & no & Pseudouridine synthase \\
\hline ypul & BSU23200 & no & rRNA pseudouridine 2633 synthase \\
\hline tils & BSU00670 & yes & tRNA ${ }^{\text {lle }}$ lysidine synthetase \\
\hline$t s a B$ & BSU05920 & yes & tRNA modification \\
\hline$t s a D$ & BSU05940 & yes & tRNA modification \\
\hline$t s a C$ & BSU36950 & no & tRNA modification \\
\hline gidA & BSU41010 & no & tRNA modification \\
\hline thdF & BSU41020 & no & GTP-binding protein, putative tRNA \\
\hline & & & modification GTPase \\
\hline $\operatorname{truA}$ & BSU01480 & no & Pseudouridylate synthase I, universally \\
\hline & & & conserved protein \\
\hline$t s a E$ & BSU05910 & no & P-loop ATPase \\
\hline $\operatorname{trmFO}$ & BSU16130 & no & tRNA:m(5)U-54 methyltransferase \\
\hline $\operatorname{mia} A$ & BSU17330 & no & tRNA isopentenylpyrophosphate transferase \\
\hline yaaJ & BSU00180 & no & tRNA-specific adenosine deaminase \\
\hline$y / y B$ & BSU15460 & no & Similar to pseudouridylate synthase \\
\hline ypsC & BSU22170 & no & rRNA modification \\
\hline \multicolumn{4}{|c|}{ Ribosome maturation/ assembly } \\
\hline$y d i D$ & BSU05930 & no & $\begin{array}{l}\text { Similar to ribosomal protein alanine } \mathrm{N} \text { - } \\
\text { acetyltransferase }\end{array}$ \\
\hline$y \mid x S$ & BSU16590 & no & $\begin{array}{l}\text { Similar to } 305 \text { ribosomal subunit maturation } \\
\text { protein }\end{array}$ \\
\hline prp & BSU27950 & yes & Maturation ofL27 \\
\hline engA & BSU22840 & yes & GTPase, ribosome $50 \mathrm{~S}$ subunit assembly \\
\hline
\end{tabular}




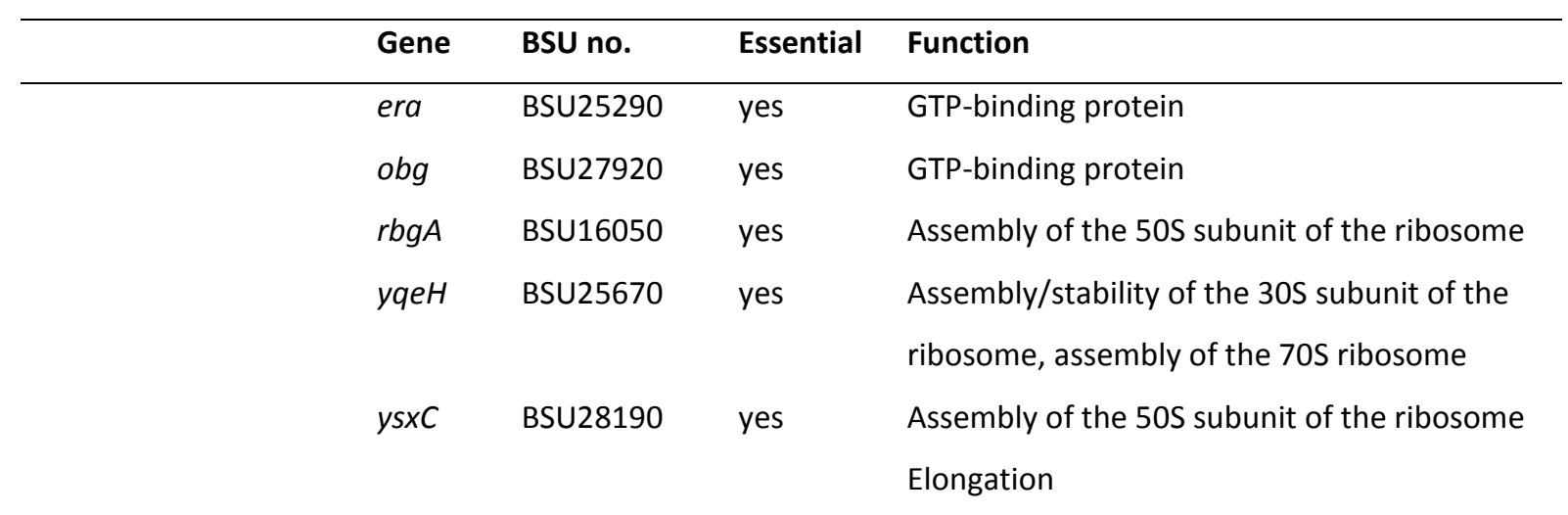

Translation factors

$\begin{array}{llll}\text { efp } & \text { BSU24450 } & \text { no } & \text { Elongation factor } \mathrm{P} \\ \text { frr } & \text { BSU16520 } & \text { yes } & \text { Ribosome recycling factor } \\ \text { fusA } & \text { BSU01120 } & \text { yes } & \text { Elongation factor } \mathrm{G} \\ \text { infA } & \text { BSU01390 } & \text { yes } & \text { Translation initiation factor IF-1 } \\ \text { infB } & \text { BSU16630 } & \text { yes } & \text { Translation initiation factor IF-2 } \\ \text { infC } & \text { BSU28870 } & \text { yes } & \text { Translation initiation factor IF-3 } \\ \text { prfA } & \text { BSU37010 } & \text { yes } & \text { Peptide chain release factor 1 } \\ \text { prfB } & \text { BSU35290 } & \text { yes } & \text { Peptide chain release factor 2 } \\ \text { tsf } & \text { BSU16500 } & \text { yes } & \text { Elongation factor Ts } \\ \text { tufA } & \text { BSU01130 } & \text { yes } & \text { Elongation factor Tu } \\ \text { lepA } & \text { BSU25510 } & \text { no } & \text { Elongation factor 4 }\end{array}$

Translation/ others

\begin{tabular}{|c|c|c|c|}
\hline map & BSU01380 & yes & Methionine aminopeptidase \\
\hline$y w k E$ & BSU37000 & no & $\begin{array}{l}\text { Similar to N5-glutamine methyltransferase } \\
\text { that modifies peptide release factors }\end{array}$ \\
\hline$r p / G B$ & BSU01090 & no & Similar to ribosomal protein $L 7$ family \\
\hline spovC & BSU00530 & yes & Putative peptidyl-tRNA hydrolase \\
\hline ssrA & $\begin{array}{l}\text { BSU_MISC_R } \\
\text { NA_55 }\end{array}$ & no & tmRNA \\
\hline$s m p B$ & BSU33600 & no & tmRNA-binding protein \\
\hline
\end{tabular}

Protein secretion

$\begin{array}{llll}\text { scr } & \text { BSU_misc_R } & \text { yes } & \text { Signal recognition particle RNA } \\ & \text { NA_2 } & & \\ f f h & \text { BSU15980 } & \text { yes } & \text { Signal recognition particle component } \\ f t s Y & \text { BSU15950 } & \text { yes } & \text { Signal recognition particle } \\ \text { yidC2 } & \text { BSU23890 } & \text { no } & \text { Sec-independent membrane protein } \\ & & & \text { translocase } \\ \sec A & \text { BSU35300 } & \text { yes } & \text { Preprotein translocase subunit (ATPase) } \\ \sec E & \text { BSU01000 } & \text { yes } & \text { Preprotein translocase subunit }\end{array}$




\begin{tabular}{|c|c|c|c|}
\hline Gene & BSU no. & Essential & Function \\
\hline $\sec Y$ & BSU01360 & yes & $\begin{array}{l}\text { Preprotein translocase subunit, universally } \\
\text { conserved protein }\end{array}$ \\
\hline $\sec G$ & BSU33630 & no & Preprotein translocase subunit \\
\hline $\operatorname{sips}$ & BSU23310 & no & Signal peptidase I \\
\hline $\operatorname{prs} A$ & BSU09950 & yes & $\begin{array}{l}\text { Protein secretion (posttranslocation } \\
\text { molecular chaperone) }\end{array}$ \\
\hline $\operatorname{csa} A$ & BSU19040 & no & $\begin{array}{l}\text { Molecular chaperone involved in protein } \\
\text { secretion }\end{array}$ \\
\hline $\lg t$ & BSU34990 & no & Prolipoprotein diacylglyceryl transferase \\
\hline IspA & BSU15450 & no & Signal peptidase II \\
\hline \multicolumn{4}{|c|}{ Proteolysis/ quality control/ chaperones } \\
\hline$h \operatorname{tr} B$ & BSU33000 & no & Serine protease \\
\hline groES & BSU06020 & yes & Chaperonin, universally conserved protein \\
\hline groEL & BSU06030 & yes & Chaperonin \\
\hline$d n a J$ & BSU25460 & no & Activation of DnaK \\
\hline dnaK & BSU25470 & no & Molecular chaperone \\
\hline $\operatorname{grp} E$ & BSU25480 & no & Activation of DnaK \\
\hline tig & BSU28230 & no & Trigger factor (prolyl isomerase) \\
\hline
\end{tabular}

Metabolism

Central carbon metabolism

Glycolysis

$\begin{array}{llll}p t s G & \text { BSU13890 } & \text { no } & \text { PTS glucose permease, ElICBA(Glc) } \\ p t s H & \text { BSU13900 } & \text { no } & \text { HPr, general component of the PTS } \\ p t s I & \text { BSU13910 } & \text { no } & \text { Enzyme I, general component of the PTS } \\ p g i & \text { BSU31350 } & \text { no } & \text { Glucose-6-phosphate isomerase } \\ p f k A & \text { BSU29190 } & \text { no } & \text { Phosphofructokinase } \\ f b a A & \text { BSU37120 } & \text { no } & \text { Fructose 1,6-bisphosphate aldolase } \\ t p i & \text { BSU33920 } & \text { no } & \text { Triose phosphate isomerase } \\ g a p A & \text { BSU33940 } & \text { yes } & \text { Glyceraldehyde-3-phosphate dehydrogenase } \\ p g k & \text { BSU33930 } & \text { No } & \text { Phosphoglycerate kinase } \\ p g m & \text { BSU33910 } & \text { yes } & \text { Phosphoglycerate mutase } \\ \text { eno } & \text { BSU33900 } & \text { yes } & \text { Enolase } \\ p y k & \text { BSU29180 } & \text { no } & \text { Pyruvate kinase } \\ p d h A & \text { BSU14580 } & \text { yes } & \text { Pyruvate dehydrogenase (E1 alpha subunit) } \\ p d h B & \text { BSU14590 } & \text { no } & \text { Pyruvate dehydrogenase (E1 beta subunit) } \\ p d h C & \text { BSU14600 } & \text { no } & \text { Pyruvate dehydrogenase (dihydrolipoamide } \\ & & & \text { acetyltransferase E2 subunit) }\end{array}$




\begin{tabular}{|c|c|c|c|}
\hline Gene & BSU no. & Essential & Function \\
\hline$p d h D$ & BSU14610 & no & $\begin{array}{l}\text { Dihydrolipoamide dehydrogenase E3 subunit } \\
\text { of both pyruvate and 2-oxoglutarate } \\
\text { dehydrogenase complexes }\end{array}$ \\
\hline \multicolumn{4}{|c|}{ Transhydrogenation cycle } \\
\hline$y t s J$ & BSU29220 & no & Malic enzyme \\
\hline mals & BSU29880 & no & Malate dehydrogenase (decarboxylating) \\
\hline \multicolumn{4}{|c|}{ Pentose phosphate pathway } \\
\hline$y k g B$ & BSU13010 & no & 6-Phosphogluconolactonase \\
\hline rpe & BSU15790 & no & Ribulose 5-phosphate 3-epimerase \\
\hline$t k t$ & BSU17890 & no & Transketolase \\
\hline$z w f$ & BSU23850 & no & Glucose-6-phosphate dehydrogenase \\
\hline gndA & BSU23860 & no & $\begin{array}{l}\text { NADP-dependent phosphogluconate } \\
\text { dehydrogenase }\end{array}$ \\
\hline$y w / F$ & BSU36920 & no & Ribose-5-phosphate isomerase \\
\hline$y w j H$ & BSU37110 & no & Transaldolase \\
\hline \multicolumn{4}{|l|}{ Recycling of acetate } \\
\hline $\operatorname{acs} A$ & BSU29680 & no & Acetyl-CoA synthetase \\
\hline \multicolumn{4}{|l|}{ Respiration/ energy } \\
\hline$n d h$ & BSU12290 & no & NADH dehydrogenase \\
\hline \multicolumn{4}{|l|}{ Cytochrome aa3 } \\
\hline $\operatorname{qoxD}$ & BSU38140 & no & Cytochrome aa3 quinol oxidase (subunit IV) \\
\hline$q 0 \times C$ & BSU38150 & no & Cytochrome aa3 quinol oxidase (subunit III) \\
\hline$q 0 \times B$ & BSU38160 & no & Cytochrome aa3 quinol oxidase (subunit I) \\
\hline$q 0 \times A$ & BSU38170 & no & Cytochrome aa3 quinol oxidase (subunit II) \\
\hline \multicolumn{4}{|c|}{ Cytochrome maturation } \\
\hline resC & BSU23130 & yes & $\begin{array}{l}\text { Part of heme translocase, required for } \\
\text { cytochrome c synthesis }\end{array}$ \\
\hline $\operatorname{res} B$ & BSU23140 & yes & $\begin{array}{l}\text { Part of heme translocase, required for } \\
\text { cytochrome c synthesis }\end{array}$ \\
\hline \multicolumn{4}{|l|}{ ATPase } \\
\hline $\operatorname{atpC}$ & BSU36800 & no & ATP synthase, $F_{1}$ (subunit epsilon) \\
\hline $\operatorname{atpD}$ & BSU36810 & no & ATP synthase, $F_{1}$ (subunit beta) \\
\hline $\operatorname{atpG}$ & BSU36820 & no & ATP synthase, $F_{1}$ (subunit gamma) \\
\hline $\operatorname{atp} A$ & BSU36830 & no & ATP synthase, $F_{1}$ (subunit alpha) \\
\hline atpH & BSU36840 & no & ATP synthase, $F_{1}$ (subunit delta) \\
\hline $\operatorname{atp} F$ & BSU36850 & no & ATP synthase, $F_{o}$ (subunit b) \\
\hline atpE & BSU36860 & no & ATP synthase, $F_{o}$ (subunit c) \\
\hline
\end{tabular}




\begin{tabular}{llll}
\hline Gene & BSU no. & Essential & Function \\
\hline atpB & BSU36870 & no & ATP synthase, Fo (subunit a) \\
atpl & BSU36880 & no & ATP synthase (subunit i)
\end{tabular}

\section{Amino Acids}

Asp, Glu

gltT BSU10220 no Major $\mathrm{H}^{+} / \mathrm{Na}^{+}$-glutamate symport protein

Arg

rocE BSU40330 no Amino acid permease

Pro

putP BSU03220 no High-affinity proline permease

$\operatorname{Trp}$

trpP BSU10010 no S protein of tryptophan ECF transporter

Met

\begin{tabular}{|c|c|c|c|}
\hline metQ & BSU32730 & no & $\begin{array}{l}\text { Methionine } A B C \text { transporter (binding } \\
\text { lipoprotein) }\end{array}$ \\
\hline metP & BSU32740 & no & Methionine $A B C$ transporter, permease \\
\hline $\operatorname{met} N$ & BSU32750 & no & $\begin{array}{l}\text { Methionine } A B C \text { transporter (ATP-binding } \\
\text { protein) }\end{array}$ \\
\hline
\end{tabular}

His

hutM BSU39390 no Histidine permease

Cys

tcyP BSU09130 no Cystine transporter

Gly

glyA BSU36900 yes Serine hydroxymethyltransferase

Ile, Val, Thr, Ser

$$
\text { bcaP BSU09460 no Branched-chain amino acid transporter }
$$

Lys

$$
\text { yvsH BSU33330 no Putative lysine transporter }
$$

\begin{tabular}{|c|c|c|c|}
\hline $\operatorname{aroA}$ & BSU29750 & no & $\begin{array}{l}\text { 3-Deoxy-D-arabino-heptulosonate 7- } \\
\text { phosphate synthase/chorismate mutase } \\
\text { isozyme } 3\end{array}$ \\
\hline $\operatorname{aroB}$ & BSU22700 & no & 3-Dehydroquinate synthase \\
\hline $\operatorname{aroc}$ & BSU23080 & no & 3-Dehydroquinate dehydratase \\
\hline $\operatorname{aroD}$ & BSU25660 & no & Shikimate dehydrogenase \\
\hline $\operatorname{aroE}$ & BSU22600 & yes & $\begin{array}{l}\text { 3-Phosphoshikimate } \\
\text { 1-carboxyvinyltransferase }\end{array}$ \\
\hline
\end{tabular}

\section{Chorismate for aromatic amino acids, menaquinone, and folate}




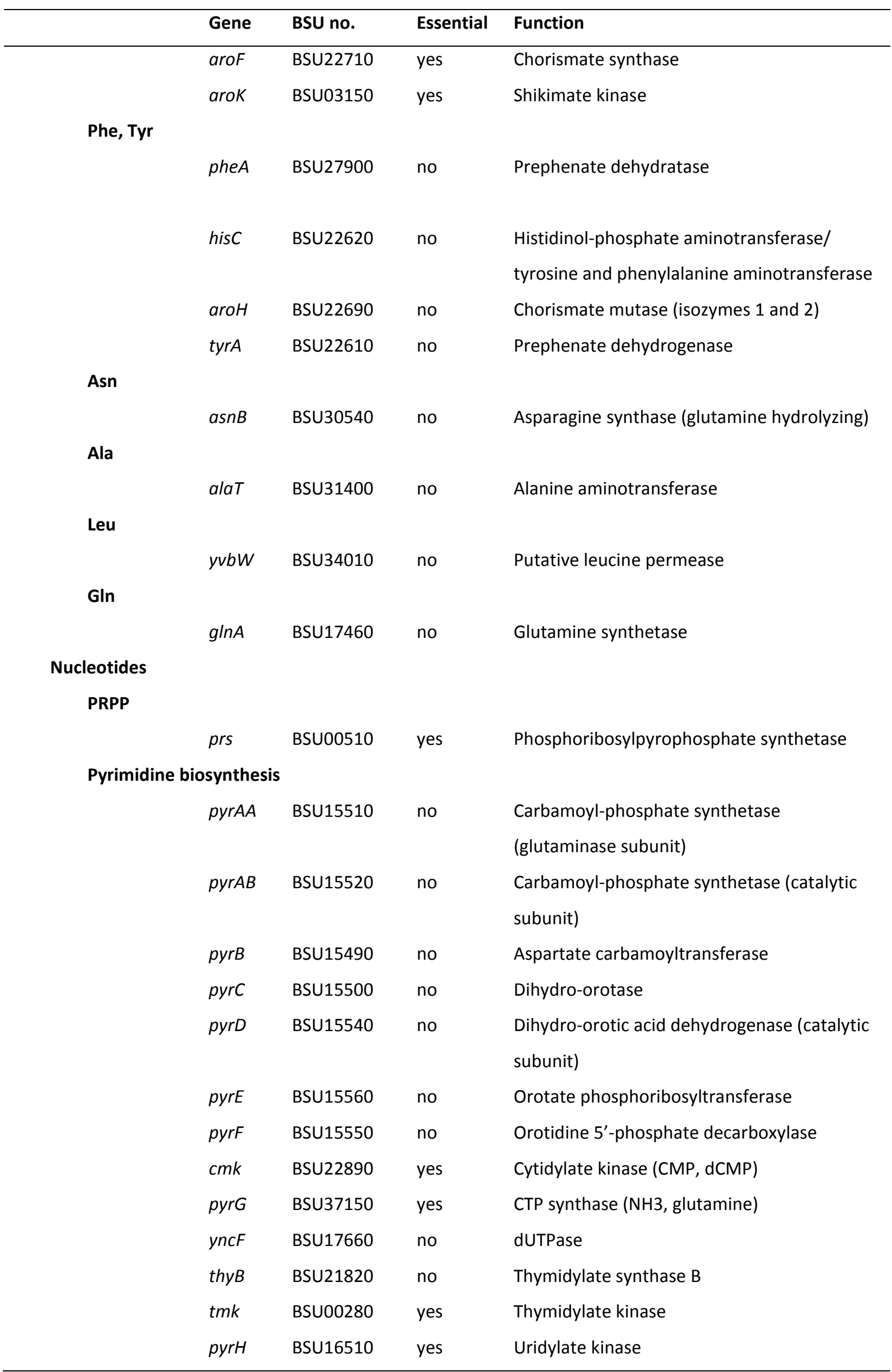




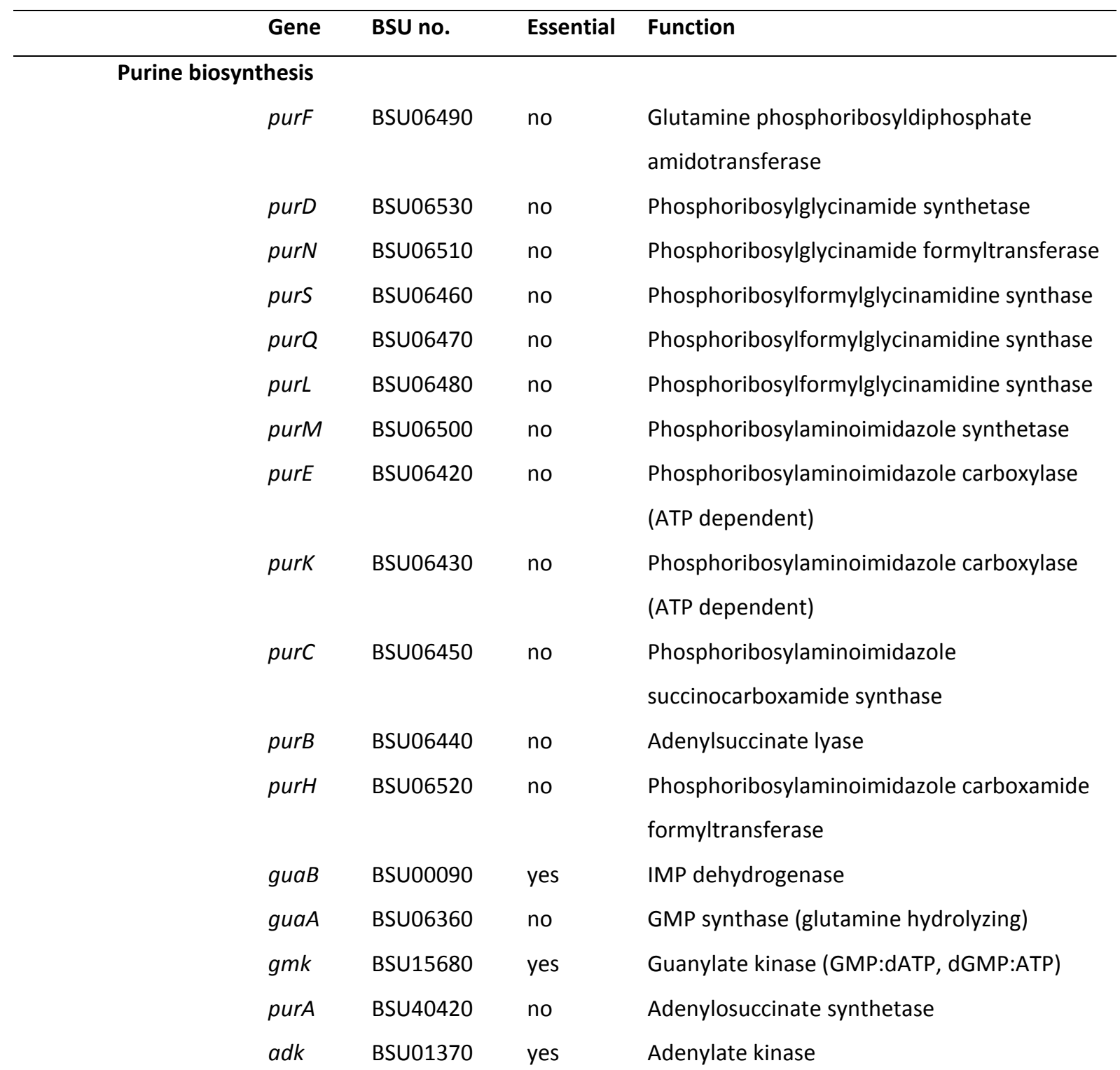

Pyrimidine/purine biosynthesis

\begin{tabular}{|c|c|c|c|}
\hline$n r d E$ & BSU17380 & yes & $\begin{array}{l}\text { Ribonucleoside diphosphate reductase (major } \\
\text { subunit) }\end{array}$ \\
\hline$n r d F$ & BSU17390 & yes & $\begin{array}{l}\text { Ribonucleoside diphosphate reductase (major } \\
\text { subunit) }\end{array}$ \\
\hline$n r d l$ & BSU17370 & yes & Ribonucleoside diphosphate reductase \\
\hline$n d k$ & BSU22730 & no & Nucleoside diphosphate kinase \\
\hline hprT & BSU00680 & yes & Hypoxanthine phosphoribosyltransferase \\
\hline
\end{tabular}

Lipids

Malonyl-CoA synthesis

$\begin{array}{llll}\text { accC } & \text { BSU24340 } & \text { yes } & \begin{array}{l}\text { Acetyl-CoA carboxylase (biotin carboxylase } \\ \text { subunit) }\end{array} \\ \text { accB } & \text { BSU24350 } & \text { yes } & \text { Acetyl-CoA carboxylase (biotin carboxyl } \\ & & \text { carrier subunit) }\end{array}$




\begin{tabular}{lllll}
\hline Gene & BSU no. & Essential & Function \\
\hline accA & BSU29200 & yes & Acetyl-CoA carboxylase (alpha subunit) \\
accD & BSU29210 & yes & Acetyl-CoA carboxylase (beta subunit) \\
birA & BSU22440 & yes & Biotin protein ligase
\end{tabular}

Acyl carrier

$\begin{array}{llll}\text { acpS BSU04620 } & \text { yes } & \begin{array}{l}\text { Acyl carrier protein synthase, 4=- } \\ \text { phosphopantetheine transferase }\end{array} \\ \text { acpA } & \text { BSU15920 yes } & \text { Acyl carrier protein }\end{array}$

Aceto-acyl-Acp synthesis

$\begin{array}{llll}\text { fabD } & \text { BSU15900 } & \text { yes } & \begin{array}{l}\text { Malonyl-CoA-acyl carrier protein } \\ \text { transacylase }\end{array} \\ \text { fabHA } & \text { BSU11330 } & \text { no } & \text { Beta-ketoacyl-acyl carrier protein synthase III }\end{array}$

$\beta$-Ketoacyl-Acp chain elongation

$\begin{array}{lll}\text { fabG } & \text { BSU15910 } & \text { yes } \\ \text { fabF } & \text { BSU11340 } & \text { yes } \\ \text { fabl } & \text { BSU11720 } & \text { no } \\ \text { ywpB } & \text { BSU36370 } & \text { yes }\end{array}$

Beta-ketoacyl-acyl carrier protein reductase

Beta-ketoacyl-acyl carrier protein synthase II

Enoyl-acyl carrier protein reductase

$\beta$-Hydroxyacyl (acyl carrier protein)

dehydratase

Phosphatidic acid synthesis

$\begin{array}{llll}\text { plsC } & \text { BSU09540 } & \text { yes } & \begin{array}{l}\text { Acyl-ACP:1-acylglycerolphosphate } \\ \text { acyltransferase }\end{array} \\ \text { plsX } & \text { BSU15890 } & \text { yes } & \begin{array}{l}\text { Acyl-ACP:phosphate acyltransferase } \\ \text { plsY }\end{array} \\ \text { BSU18070 } & \text { yes } & \begin{array}{l}\text { Acylphosphate:glycerol-phosphate } \\ \text { acyltransferase }\end{array} \\ & & & \text { Glycerol-3-phosphate dehydrogenase (NAD) }\end{array}$

Phosphatidylglycerol phosphate synthesis

$\begin{array}{llll}c d s A & \text { BSU16540 } & \text { yes } & \text { Phosphatidate cytidylyltransferase } \\ \text { pgsA } & \text { BSU16920 } & \text { yes } & \text { Phosphatidylglycerophosphate synthase }\end{array}$

Phosphate

pit BSU12840 no Low-affinity phosphate transporter

Cofactors

ECF transporter (general comonent) for riboflavin, biotin, thaimine, tryptophan

$\begin{array}{cccl}y b x A & \text { BSU01450 } & \text { no } & \begin{array}{l}\text { ATP-binding A1 component of ECF } \\ \text { transporters }\end{array} \\ \text { ybaE } & \text { BSU01460 } & \text { no } & \begin{array}{l}\text { ATP-binding A2 component of ECF } \\ \text { transporters }\end{array} \\ & & & \text { Transmembrane T component ofECF } \\ y b a F & \text { BSU01470 } & \text { no } & \text { transporters }\end{array}$




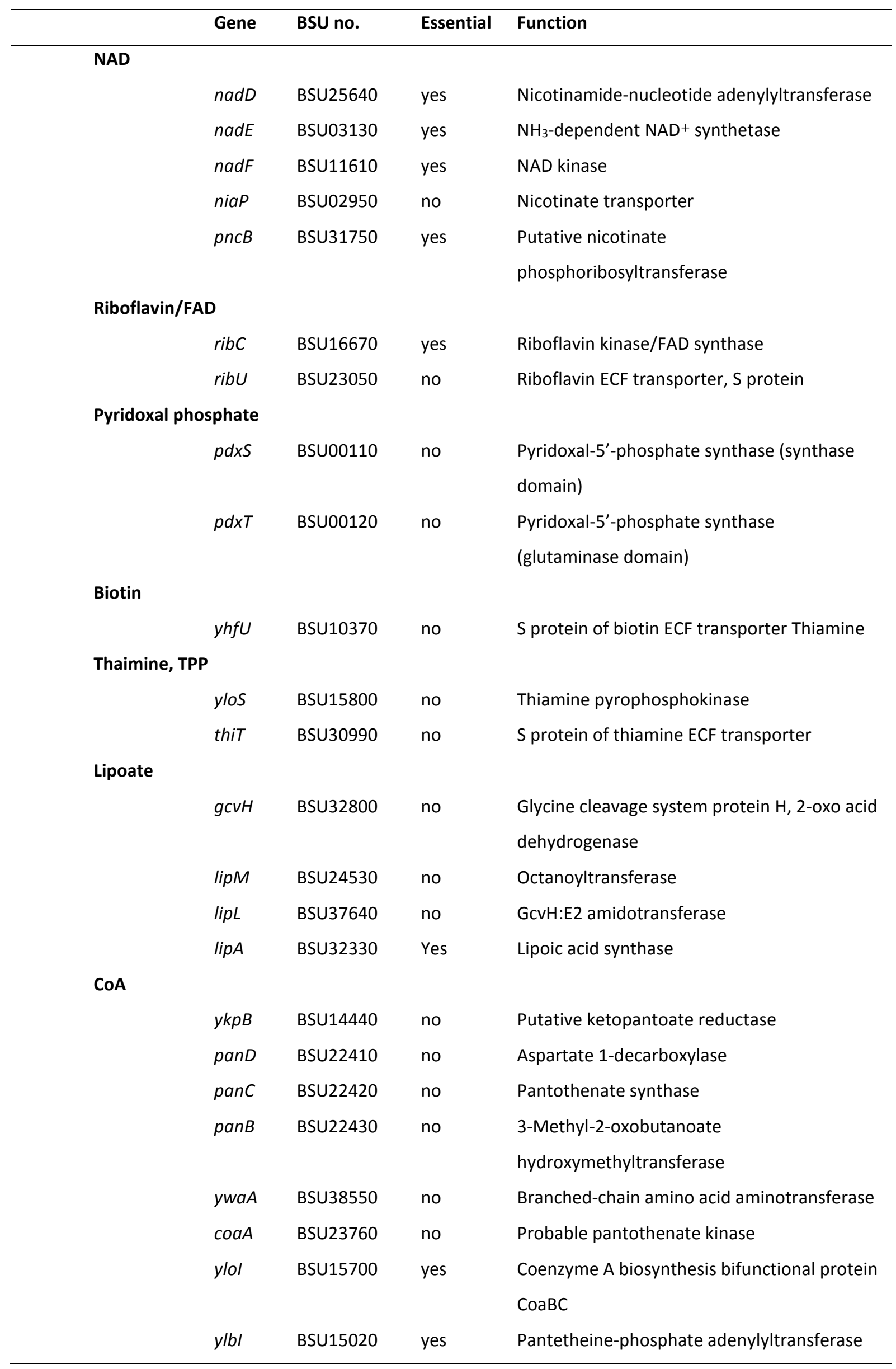




\begin{tabular}{lllll}
\hline & Gene & BSU no. & Essential & Function \\
\hline SAM & ytaG & BSU29060 & yes & Dephospho-CoA kinase \\
& metK & BSU30550 & yes & S-Adenosylmethionine synthetase
\end{tabular}

Folate

$\begin{array}{llll}\text { folE } & \text { BSU22780 } & \text { yes } & \text { GTP cyclohydrolase I } \\ \text { phoB } & \text { BSU05740 } & \text { no } & \text { Alkaline phosphatase A } \\ \text { folB } & \text { BSU00780 } & \text { yes } & \text { Dihydroneopterin aldolase } \\ \text { folK } & \text { BSU00790 } & \text { yes } & \begin{array}{l}\text { 2-Amino-4-hydroxy-6-hydroxymethyl- } \\ \text { dihydropteridine diphosphokinase }\end{array} \\ \text { sul } & \text { BSU00770 } & \text { yes } & \text { Dihydropteroate synthase } \\ \text { folC } & \text { BSU28080 } & \text { yes } & \text { Folyl-polyglutamate synthetase } \\ d f r A & \text { BSU21810 } & \text { yes } & \text { Dihydrofolate reductase } \\ \text { pabB } & \text { BSU00740 } & \text { no } & \text { p-Aminobenzoate synthase (subunit A) } \\ \text { pabA } & \text { BSU00750 } & \text { no } & \text { p-Aminobenzoate synthase (subunit B)/ } \\ & & & \text { anthranilate synthase (subunit II) } \\ \text { pabC } & \text { BSU00760 } & \text { no } & \text { Aminodeoxychorismate lyase } \\ g s a B & \text { BSU08710 } & \text { no } & \text { Formate dehydrogenase }\end{array}$

Heme biosynthesis

\begin{tabular}{|c|c|c|c|}
\hline hemE & BSU10120 & no & Glutamate-1-semialdehyde aminotransferase \\
\hline hemH & BSU10130 & no & $\begin{array}{l}\text { Uroporphyrinogen decarboxylase } \\
\text { (uroporphyrinogen III) }\end{array}$ \\
\hline hemY & BSU10140 & no & Ferrochelatase \\
\hline $\operatorname{cta} A$ & BSU14870 & no & Protoporphyrinogen IX oxidase \\
\hline $\operatorname{cta} B$ & BSU14880 & no & Heme A synthase \\
\hline hemL & BSU28120 & no & Heme O synthase (major enzyme) \\
\hline hemB & BSU28130 & no & Glutamate-1-semialdehyde aminotransferase \\
\hline hemD & BSU28140 & no & Porphobilinogen synthase \\
\hline hemC & BSU28150 & no & Uroporphyrinogen III synthase \\
\hline hemX & BSU28160 & no & Hydroxymethylbilane synthase \\
\hline hemA & BSU28170 & no & Glutamyl-tRNA reductase \\
\hline hemQ & BSU37670 & no & Heme-binding protein \\
\hline menA & BSU38490 & yes & $\begin{array}{l}\text { Probable 1,4-dihydroxy-2-naphthoate } \\
\text { octaprenyltransferase }\end{array}$ \\
\hline menH & BSU22750 & yes & Menaquinone biosynthesis methyltransferase \\
\hline menc & BSU30780 & yes & O-Succinylbenzoate-CoA synthase \\
\hline menE & BSU30790 & yes & O-Succinylbenzoate-CoA ligase \\
\hline
\end{tabular}




\begin{tabular}{lllll}
\hline Gene & BSU no. & Essential & Function \\
\hline menB & BSU30800 & yes & Naphthoate synthase \\
menD & BSU30820 & yes & 2-Succinyl-6-hydroxy-2,4-cyclohexadiene-1- \\
& & & carboxylate synthase/ 2-oxoglutarate \\
& & & decarboxylase \\
ytxM & BSU30810 & no & Similar to prolyl aminopeptidase \\
menF & BSU30830 & no & Menaquinone-specific isochorismate \\
& & &
\end{tabular}

Metals and iron-sulfur clusters

Sodium export

$\begin{array}{llll}m r p A & \text { BSU31600 } & \text { yes } & \mathrm{Na}^{+} / \mathrm{H}^{+} \text {antiporter subunit } \\ m r p B & \text { BSU31610 } & \text { yes } & \mathrm{Na}^{+} / \mathrm{H}^{+} \text {antiporter subunit } \\ m r p C & \text { BSU31620 } & \text { yes } & \mathrm{Na}^{+} / \mathrm{H}^{+} \text {antiporter subunit } \\ m r p D & \text { BSU31630 } & \text { yes } & \mathrm{Na}^{+} / \mathrm{H}^{+} \text {antiporter subunit } \\ m r p E & \text { BSU31640 } & \text { yes } & \mathrm{Na}^{+} / \mathrm{H}^{+} \text {antiporter subunit } \\ m r p F & \text { BSU31650 } & \text { yes } & \mathrm{Na}^{+} / \mathrm{H}^{+} \text {antiporter subunit } \\ m r p G & \text { BSU31660 } & \text { yes } & \mathrm{Na}^{+} / \mathrm{H}^{+} \text {antiporter subunit }\end{array}$

\section{Potassium}

kimA BSU04320 no High-affinity potassium transporter

Iron

\begin{tabular}{|c|c|c|c|}
\hline efeB & BSU38260 & no & Heme peroxidase in elemental iron uptake \\
\hline efeO & BSU38270 & no & $\begin{array}{l}\text { Lipoprotein, elemental iron uptake system } \\
\text { (binding protein) }\end{array}$ \\
\hline efeU & BSU38280 & no & Elemental iron uptake system (permease) \\
\hline yfmF & BSU07490 & no & $\begin{array}{l}\text { Iron/citrate ABC transporter (ATP-binding } \\
\text { protein) }\end{array}$ \\
\hline yfmE & BSU07500 & no & Iron/citrate $\mathrm{ABC}$ transporter (permease) \\
\hline yfmD & BSU07510 & no & Iron/citrate $\mathrm{ABC}$ transporter (permease) \\
\hline$y f m C$ & BSU07520 & no & Iron/citrate $A B C$ transporter (binding protein) \\
\hline yhfQ & BSU10330 & no & $\begin{array}{l}\text { Iron/citrate } A B C \text { transporter (solute-binding } \\
\text { protein) }\end{array}$ \\
\hline pfet & BSU13850 & no & Iron efflux pump \\
\hline
\end{tabular}

Magnesium

$\begin{array}{llll}m g t E & \text { BSU13300 } & \text { yes } & \text { Primary magnesium transporter } \\ m n t H & \text { BSU04360 } & \text { no } & \text { Manganese transporter (proton symport) }\end{array}$

Zinc

znuA BSU02850 no ABC transporter for zinc (binding protein) 


\begin{tabular}{|c|c|c|c|c|}
\hline & Gene & BSU no. & Essential & Function \\
\hline & znuC & BSU02860 & no & $\begin{array}{l}A B C \text { transporter for zinc (ATP-binding } \\
\text { protein) }\end{array}$ \\
\hline & $z n u B$ & BSU02870 & no & $A B C$ transporter for zinc (permease) \\
\hline \multicolumn{5}{|l|}{ Copper } \\
\hline & $y c n J$ & BSU03950 & no & Copper transporter \\
\hline \multicolumn{5}{|l|}{ Fe-S cluster } \\
\hline & sufB & BSU32670 & yes & Synthesis of Fe-S clusters \\
\hline & sufU & BSU32680 & yes & Iron-sulfur cluster scaffold protein \\
\hline & sufD & BSU32700 & yes & Synthesis of Fe-S clusters \\
\hline & sufs & BSU32690 & yes & Cysteine desulfurase \\
\hline & sufC & BSU32710 & yes & $A B C$ transporter (ATP-binding protein) \\
\hline & fra & BSU05750 & no & Frataxin-like protein \\
\hline & yutl & BSU32220 & no & Putative iron-sulfur scaffold protein \\
\hline
\end{tabular}

\section{Cell division}

Cell wall synthesis

Synthesis of D-glutamate

racE BSU28390 yes Glutamate racemase

Synthesis of D-Ala-D-Ala

$\begin{array}{llll}\text { alr } & \text { BSU04640 } & \text { yes } & \text { Alanine racemase } \\ d d l & \text { BSU04560 } & \text { yes } & \text { D-Alanine-D-alanine ligase }\end{array}$

Synthesis of $\mathbf{m}$-diaminopimelate

$\begin{array}{llll}\text { dapG } & \text { BSU16760 } & \text { yes } & \text { Aspartokinase I (alpha and beta subunits) } \\ \text { asd } & \text { BSU16750 } & \text { yes } & \text { Aspartate-semialdehyde dehydrogenase } \\ \text { dapA } & \text { BSU16770 } & \text { yes } & \text { Dihydrodipicolinate synthase } \\ \text { dapB } & \text { BSU22490 } & \text { yes } & \text { Dihydrodipicolinate reductase (NADPH) } \\ y k u Q & \text { BSU14180 } & \text { yes } & \text { Similar to tetrahydrodipicolinate succinylase } \\ \text { patA } & \text { BSU14000 } & \text { yes } & \text { Aminotransferase } \\ y k u R & \text { BSU14190 } & \text { yes } & \text { N-Acetyl-diaminopimelate deacetylase } \\ \text { dapF } & \text { BSU32170 } & \text { yes } & \text { Diaminopimelate epimerase }\end{array}$

Isoprenoid biosynthesis

$\begin{array}{llll}d x s & \text { BSU24270 } & \text { yes } & \begin{array}{l}\text { 1-Deoxyxylulose-5-phosphate synthase } \\ \text { ispC }\end{array} \\ \text { BSU16550 } & \text { yes } & \begin{array}{l}\text { 1-Deoxy-D-xylulose-5-phosphate } \\ \text { reductoisomerase }\end{array} \\ \text { ispD } & \text { BSU00900 } & \text { yes } & \begin{array}{l}\text { 2-C-Methyl-D-erythritol 4-phosphate } \\ \text { cytidylyltransferase }\end{array}\end{array}$




\begin{tabular}{|c|c|c|c|}
\hline Gene & BSU no. & Essential & Function \\
\hline ispE & BSU00460 & yes & $\begin{array}{l}\text { 4-Diphosphocytidyl-2-C-methyl-D-erythritol } \\
\text { kinase }\end{array}$ \\
\hline$i s p F$ & BSU00910 & yes & $\begin{array}{l}\text { 2-C-Methyl-D-erythritol-2,4- } \\
\text { cyclodiphosphate synthase }\end{array}$ \\
\hline ispG & BSU25070 & yes & $\begin{array}{l}\text { Similar to peptidoglycan acetylation, 1- } \\
\text { hydroxy-2-methyl-2-(E)-butenyl-4- } \\
\text { diphosphate synthase }\end{array}$ \\
\hline ispH & BSU25160 & yes & $\begin{array}{l}\text { (E)-4-Hydroxy-3-methylbut-2-enyl } \\
\text { diphosphate reductase }\end{array}$ \\
\hline fni & BSU22870 & no & Isopentenyl diphosphate isomerase \\
\hline \multicolumn{4}{|c|}{ Undecaprenyl phosphate biosynthesis } \\
\hline yqiD & BSU24280 & no & Geranyltransferase \\
\hline uppS & BSU16530 & yes & $\begin{array}{l}\text { Probable undecaprenyl pyrophosphate } \\
\text { synthetase }\end{array}$ \\
\hline$b c r C$ & BSU36530 & no & Undecaprenyl pyrophosphate phosphatase \\
\hline hepT & BSU22740 & yes & $\begin{array}{l}\text { Heptaprenyl diphosphate synthase } \\
\text { component II }\end{array}$ \\
\hline heps & BSU22760 & yes & $\begin{array}{l}\text { Heptaprenyl diphosphate synthase } \\
\text { component I }\end{array}$ \\
\hline \multicolumn{4}{|c|}{ Peptidoglycan biosynthesis } \\
\hline glms & BSU01780 & yes & $\begin{array}{l}\text { Glutamine:fructose-6-phosphate } \\
\text { transaminase }\end{array}$ \\
\hline$g \operatorname{lmM}$ & BSU01770 & yes & Phosphoglucosamine mutase \\
\hline$g c a D$ & BSU00500 & yes & UDP-N-acetylglucosamine pyrophosphorylase \\
\hline murAA & BSU36760 & yes & $\begin{array}{l}\text { UDP-N-acetylglucosamine 1- } \\
\text { carboxyvinyltransferase }\end{array}$ \\
\hline murB & BSU15230 & yes & $\begin{array}{l}\text { UDP-N-acetylenolpyruvoylglucosamine } \\
\text { reductase }\end{array}$ \\
\hline murC & BSU29790 & yes & UDP-N-acetylmuramoyl-L-alanine synthetase \\
\hline murD & BSU15200 & yes & $\begin{array}{l}\text { UDP-N-acetylmuramoyl-L-alanyl-D-glutamate } \\
\text { synthetase }\end{array}$ \\
\hline murE & BSU15180 & yes & $\begin{array}{l}\text { UDP-N-acetylmuramoyl-L-alanyl-D-glutamyl- } \\
\text { meso-2,6-diaminopimelate synthetase }\end{array}$ \\
\hline murF & BSU04570 & yes & $\begin{array}{l}\text { UDP-N-acetylmuramoyl-L-alanyl-D-glutamyl- } \\
\text { meso-2,6-diaminopimeloyl-D-alanyl-D-alanine } \\
\text { synthetase }\end{array}$ \\
\hline
\end{tabular}




\begin{tabular}{lllll}
\hline Gene & BSU no. & Essential & Function \\
\hline mraY & BSU15190 & yes & Phospho-N-acetylmuramoyl-pentapeptide \\
& & & transferase (meso-2,6-diaminopimelate) \\
murG & BSU15220 & yes & UDP-N-acetylglucosamine-N-acetylmuramyl- \\
& & & (pentapeptide)pyrophosphoryl-undecaprenol \\
& & & N-acetylglucosamine transferase \\
amj & BSU04230 & no & Lipid II flipase
\end{tabular}

Peptidoglycan polymerization

$\begin{array}{cl}\text { ponA BSU22320 no } & \text { Penicillin-binding protein } 1 \mathrm{~A} / 1 \mathrm{~B} \\ & \text { Penicillin-binding }\end{array}$

PG cross-links, cell separation

\begin{tabular}{|c|c|c|c|}
\hline$p b p B$ & BSU15160 & yes & Penicillin-binding protein 2B \\
\hline$p b p A$ & BSU25000 & no & Penicillin-binding protein $2 \mathrm{~A}$ \\
\hline lytE & BSU09420 & no & $\begin{array}{l}\text { Cell wall hydrolase (major autolysin) for cell } \\
\text { elongation and separation }\end{array}$ \\
\hline lytF & BSU09370 & no & $\begin{array}{l}\text { Gamma-D-glutamate-meso-diaminopimelate } \\
\text { muropeptidase (major autolysin) }\end{array}$ \\
\hline
\end{tabular}

Wall teichoic acid

\begin{tabular}{|c|c|c|c|}
\hline $\operatorname{tag} O$ & BSU35530 & yes & $\begin{array}{l}\text { Undecaprenyl phosphate-GIcNAc-1- } \\
\text { phosphate transferase }\end{array}$ \\
\hline mnaA & BSU35660 & yes & UDP-N-acetylglucosamine 2-epimerase \\
\hline $\operatorname{tag} A$ & BSU35750 & yes & UDP-N-acetyl-D-mannosamine transferase \\
\hline $\operatorname{tag} B$ & BSU35760 & yes & $\begin{array}{l}\text { Putative CDP-glycerol:glycerol phosphate } \\
\text { glycerophosphotransferase }\end{array}$ \\
\hline $\operatorname{tag} D$ & BSU35740 & yes & Glycerol-3-phosphate cytidylyltransferase \\
\hline $\operatorname{tag} F$ & BSU35720 & yes & $\begin{array}{l}\text { CDP-glycerol:polyglycerol phosphate } \\
\text { glycerophosphotransferase }\end{array}$ \\
\hline $\operatorname{tagH}$ & BSU35700 & yes & $\begin{array}{l}\text { ABC transporter for teichoic acid } \\
\text { translocation (ATP-binding protein) }\end{array}$ \\
\hline $\operatorname{tag} G$ & BSU35710 & yes & $\begin{array}{l}\text { ABC transporter for teichoic acid } \\
\text { translocation (permease) }\end{array}$ \\
\hline $\operatorname{tag} U$ & BSU35650 & no & $\begin{array}{l}\text { Phosphotransferase, attachment of anionic } \\
\text { polymers to peptidoglycan }\end{array}$ \\
\hline
\end{tabular}

Lipoteichoic acid

$\begin{array}{llll}d g k B & \text { BSU06720 } & \text { yes } & \text { Diacylglycerol kinase } \\ \text { pgcA } & \text { BSU09310 } & \text { no } & \text { Alpha-phosphoglucomutase } \\ g t a B & \text { BSU35670 } & \text { no } & \text { UTP-glucose-1-phosphate uridylyltransferase }\end{array}$




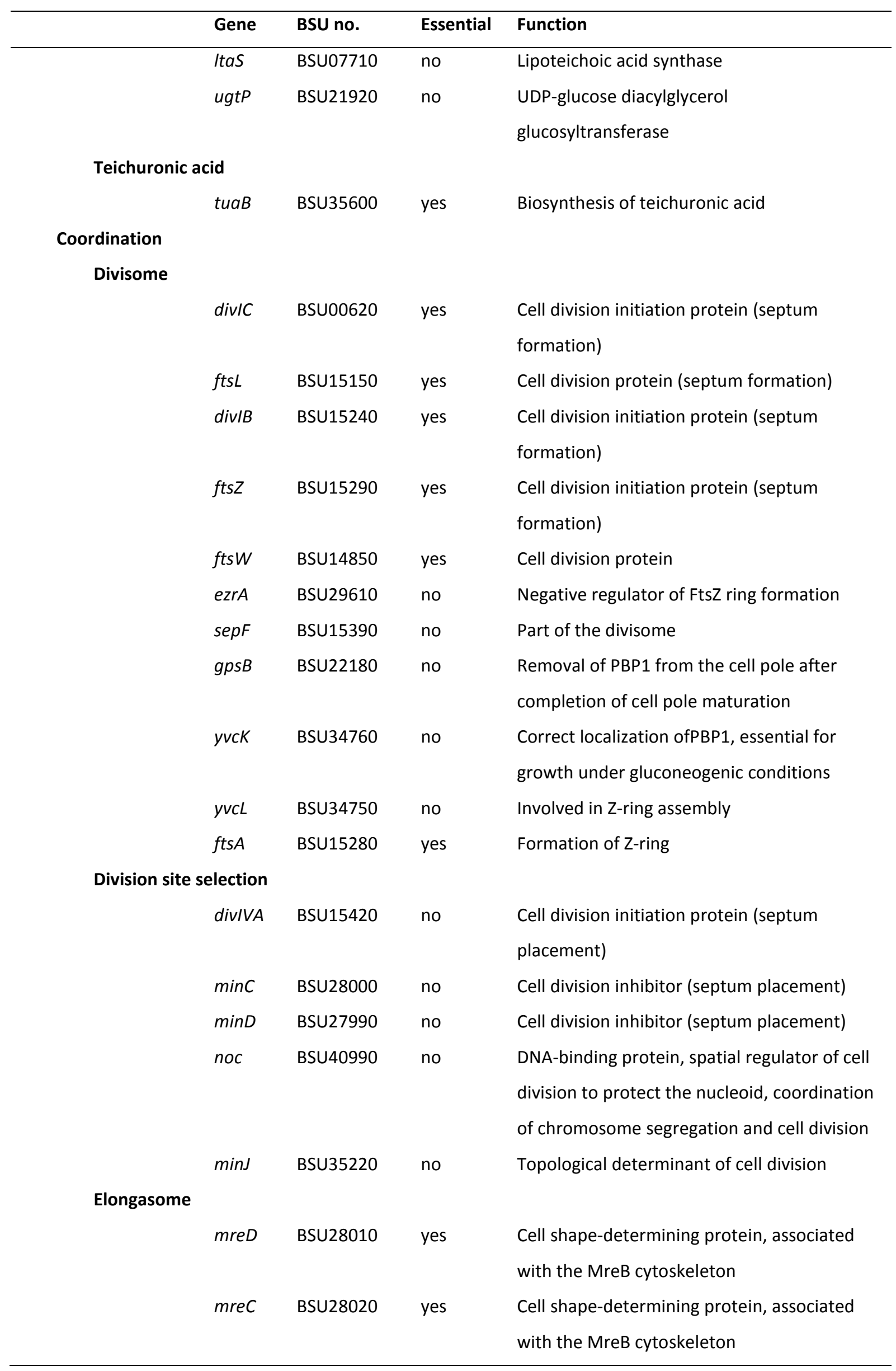




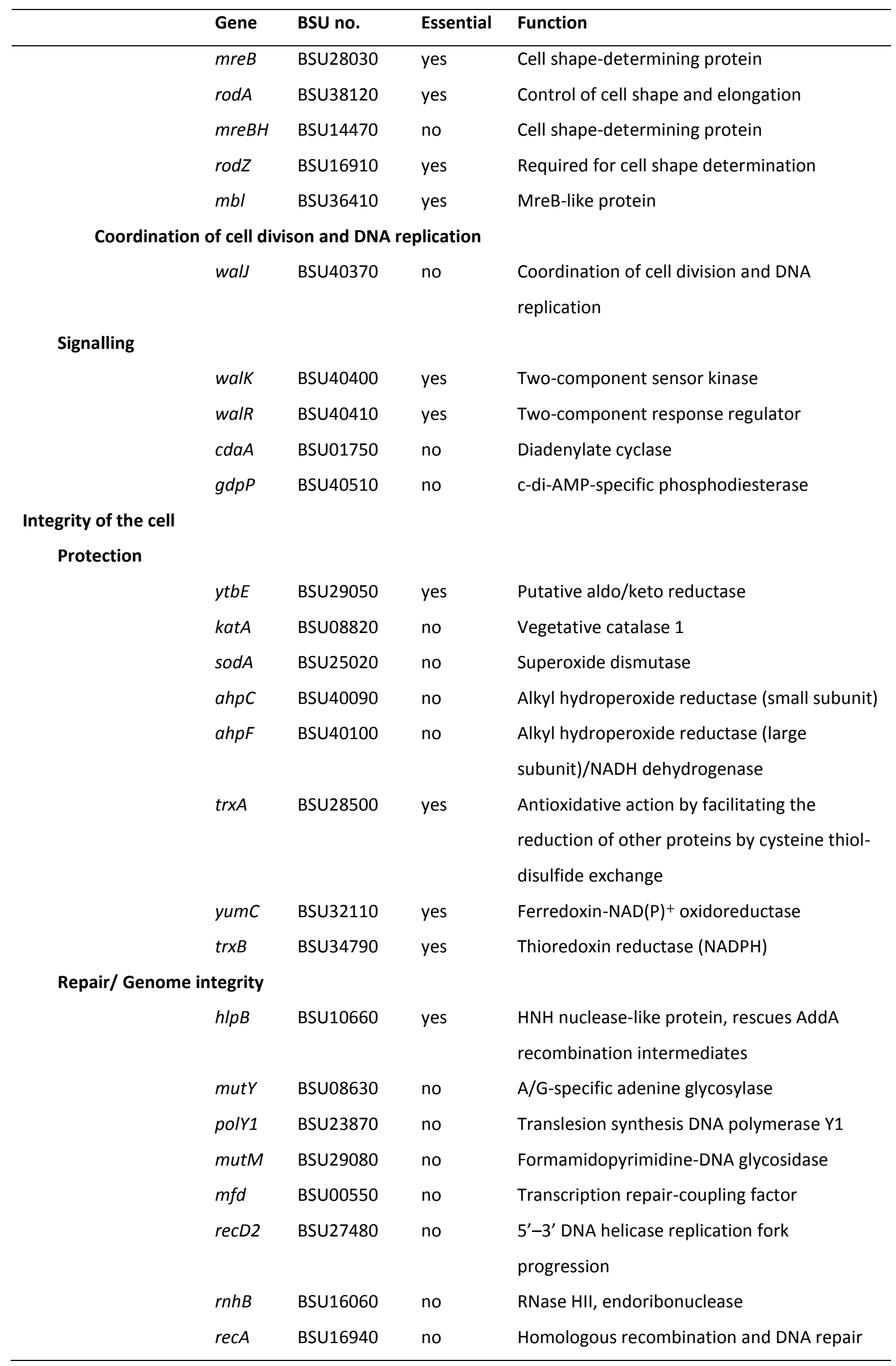




\begin{tabular}{|c|c|c|c|c|}
\hline & Gene & BSU no. & Essential & Function \\
\hline & pcrA & BSU06610 & yes & ATP-dependent DNA helicase \\
\hline \multicolumn{5}{|l|}{ Other/ unknown } \\
\hline & ppaC & BSU40550 & yes & Inorganic pyrophosphatase \\
\hline & ylan & BSU14840 & yes & Unknown \\
\hline & yitl & BSU11000 & no & Unknown \\
\hline & yitw & BSU11160 & no & Unknown \\
\hline & $y q h Y$ & BSU24330 & no & Unknown \\
\hline & $y k w C$ & BSU13960 & no & Putative beta-hydroxy acid dehydrogenase \\
\hline & $y / b N$ & BSU15070 & no & Unknown \\
\hline & ypfD & BSU22880 & no & Similar to ribosomal protein S1 \\
\hline & yugl & BSU31390 & no & Similar to polyribonucleotide \\
\hline & & & & nucleotidyltransferase \\
\hline & flot & BSU31010 & no & Similar to flotillin 1 , orchestration of \\
\hline & & & & physiological processes in lipid microdomains \\
\hline & yyaF & BSU40920 & no & GTP-binding protein/GTPase \\
\hline & yezG & BSU06811 & yes & Antitioxin \\
\hline & yneF & BSU17910 & yes & Unknown \\
\hline & yqeG & BSU25680 & yes & Unknown \\
\hline & wapl & BSU39220 & yes & Immunity protein \\
\hline & $y x x D$ & BSU39290 & yes & Antitioxin \\
\hline
\end{tabular}




\section{Curriculum Vitae}

\section{Personal information}

$\begin{array}{ll}\text { Name } & \text { Anika Klewing } \\ \text { Date of birth } & 5^{\text {th }} \text { November, 1990 (Nordhorn, Germany) } \\ \text { Nationality } & \text { German } \\ \text { Contact } & \text { anika_klewing@web.de }\end{array}$

\section{Education}

2015-2019

Ph.D. thesis

Georg-August-Universität Göttingen, Prof. Dr. Jörg Stülke at the GGNB program Microbiology and Biochemistry

Title of thesis: "MiniBacillus- the construction of a minimal organism"

2013-2015

Master of Science, Microbiology \& Biochemistry

Georg-August-Universität Göttingen, Prof. Dr. Jörg Stülke

Title of thesis: "Investigation of the impactof YqhY on the acetyl-CoA

carboxylase activity in Bacillus subtilis"

2010-2013

Bachelor of Science, Biology

Georg-August-Universität Göttingen, Prof. Dr. Jörg Stülke

Area of specification: Molecular life sciences

Title of thesis: "Genomminimierung in Bacillus subtilis: Konsequenzen des Verlustes des Citratzyklus"

2003-2010

\section{Higher School Certificate}

Franziskus Gymnasium, Lingen 